\title{
Long Term Evolution - Orthogonal Frequency Division Multiplexing Time and Frequency Synchronization Techniques
}

\author{
Ky-Bao Huu Ho, B. Eng.
}

A thesis submitted to the Faculty of Graduate and Postdoctoral Studies in partial fulfillment of the requirements for the degree of

Master of Applied Science

in

Electrical and Computer Engineering

Ottawa-Carleton Institute for Electrical and Computer Engineering

Carleton University

Ottawa, Ontario

C2012 Ky-Bao Huu Ho 
Library and Archives

Canada

Published Heritage

Branch

395 Wellington Street

Ottawa ON K1A ON4

Canada
Bibliothèque et

Archives Canada

Direction du

Patrimoine de l'édition

395 , rue Wellington

Ottawa ON K1A ON4

Canada
Your file Votre référence

ISBN: 978-0-494-91572-1

Our file Notre référence

ISBN: 978-0-494-91572-1
NOTICE:

The author has granted a nonexclusive license allowing Library and Archives Canada to reproduce, publish, archive, preserve, conserve, communicate to the public by telecommunication or on the Internet, loan, distrbute and sell theses worldwide, for commercial or noncommercial purposes, in microform, paper, electronic and/or any other formats.

The author retains copyright ownership and moral rights in this thesis. Neither the thesis nor substantial extracts from it may be printed or otherwise reproduced without the author's permission.
AVIS:

L'auteur a accordé une licence non exclusive permettant à la Bibliothèque et Archives Canada de reproduire, publier, archiver, sauvegarder, conserver, transmettre au public par télécommunication ou par l'Internet, prêter, distribuer et vendre des thèses partout dans le monde, à des fins commerciales ou autres, sur support microforme, papier, électronique et/ou autres formats.

L'auteur conserve la propriété du droit d'auteur et des droits moraux qui protege cette thèse. $\mathrm{Ni}$ la thèse ni des extraits substantiels de celle-ci ne doivent être imprimés ou autrement reproduits sans son autorisation.
In compliance with the Canadian Privacy Act some supporting forms may have been removed from this thesis.

While these forms may be included in the document page count, their removal does not represent any loss of content from the thesis.
Conformément à la loi canadienne sur la protection de la vie privée, quelques formulaires secondaires ont été enlevés de cette thèse.

Bien que ces formulaires aient inclus dans la pagination, il n'y aura aucun contenu manquant. 


\begin{abstract}
This research thesis is an experimental study to investigate several state-of-the-art spectrum sensing methods and latest research on Long Term Evolution (LTE) - Orthogonal Frequency Division Multiplexing (OFDM) time and frequency synchronization techniques for frequency-agile radios. A frequency-agile radio is a secondary user that dynamically accesses available spectrum in an unlicensed manner when that spectrum is unoccupied by a primary user. Synchronization in both time and frequencydomain is a critical and crucial problem in OFDM systems, since a frequency error results in inter-carrier interference (ICI). The goal in symbol timing offset (STO) estimation is to find a place to start the $N$-point FFT for demodulating an OFDM symbol. The goal in carrier frequency offset (CFO) estimation is to preserve the orthogonal properties of the sub-carriers. A proposed OFDM synchronization algorithm application proof-of-concept based on the Zadoff-Chu CAZAC (Constant Amplitude Zero Correlation) pilot sequence for LTE/LTE-Advanced has been developed, presented, and demonstrated.
\end{abstract}




\section{Acknowledgments}

First and foremost, I want to thank first my parents and particularly my family for their love, patience, unwavering support, and encouragement throughout my graduate study. I would like to express my sincere gratitude to my professor and graduate studies advisor Dr. Jim Wight for his advice, guidance, support, proof reading the manuscript, and giving me invaluable comments and suggestions in order to make this thesis possible. I would like to thank Mr. Martial Dufour and Dr. Francois Patenaude of Communications Research Centre (CRC) Ottawa, for given me the opportunity, where this work was carried out. Since ability is of little account without opportunity. I would like to acknowledge Dr. John Lodge of CRC for providing lab equipment materials, financial support, and facilitation during my study as well as my work. Finally, a special thanks to Dr. Daniel Boudreau of CRC, for proof reading the manuscript, as well as his assistance and help in making this thesis better. 


\section{Table of Contents}

ABSTRACT $\ldots \ldots \ldots \ldots \ldots \ldots$

ACKNOWLEDGMENTS

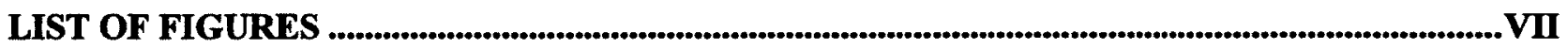

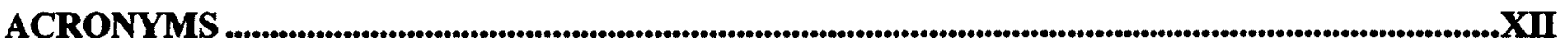

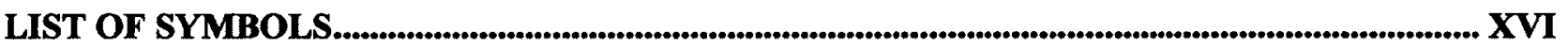

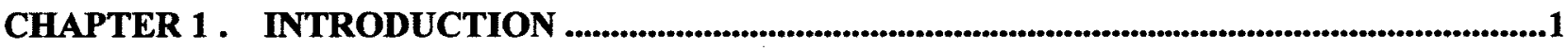

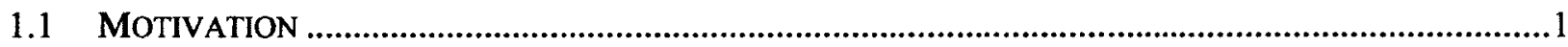

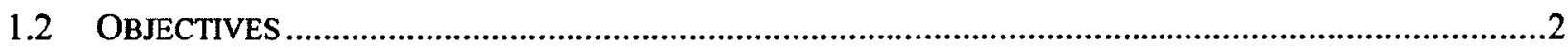

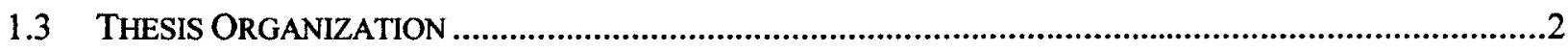

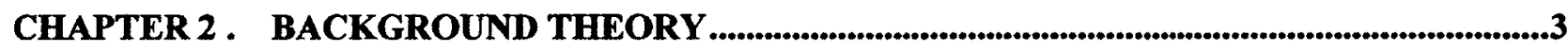

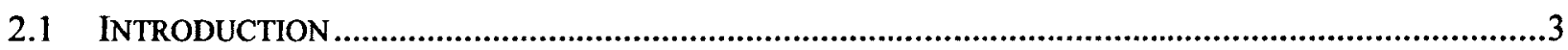

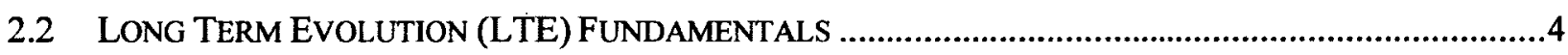

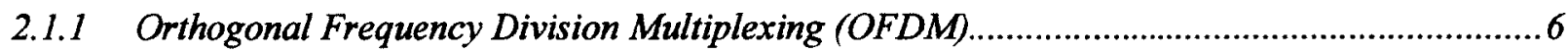

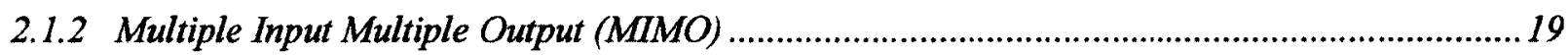

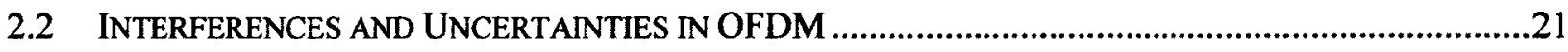

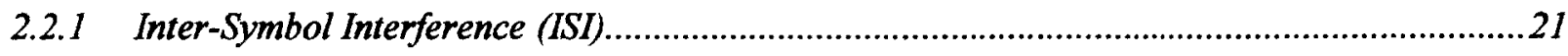

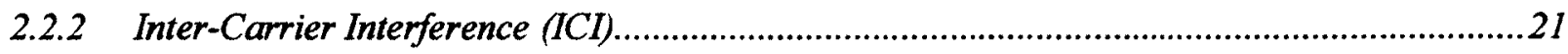

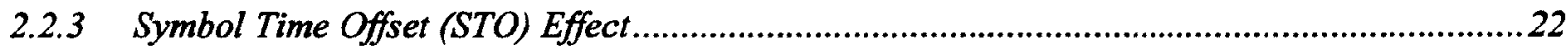

2.2.4 Carrier Frequency Offset (CFO) Effect........................................................................22

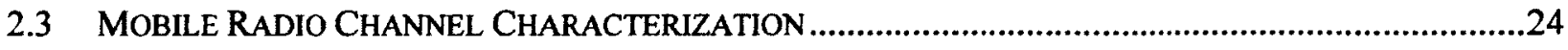

2.3.1 Small-Scale Fading (Frequency-Dependent) ............................................................24

2.3.2 Large-Scale Fading (Frequency-Independent)................................................................30 
2.3.3 Channel Coherence, Spread, Dispersion and Selectivity ...................................................31

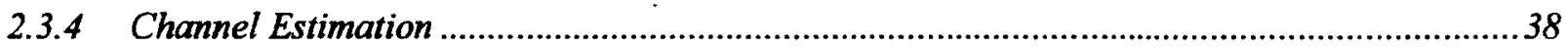

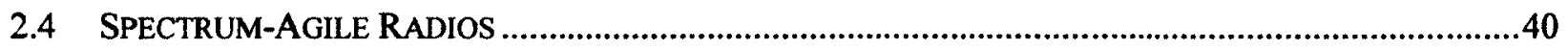

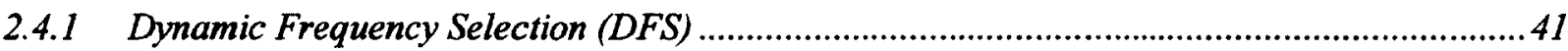

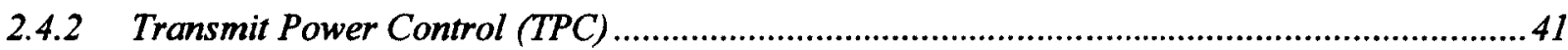

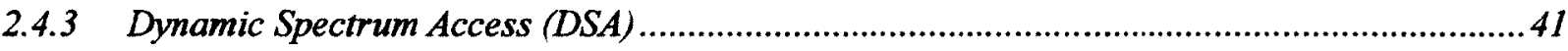

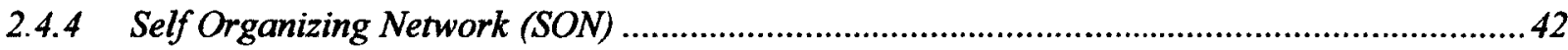

2.4.5 Coexistence and Self-Coexistence Mechanism ............................................................

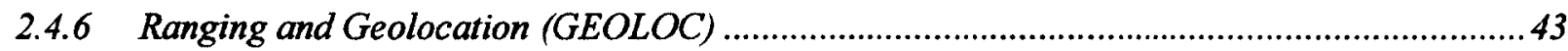

2.4.7 Vertical Handover (VHO)/Media Independent Handover (MIH) .....................................44

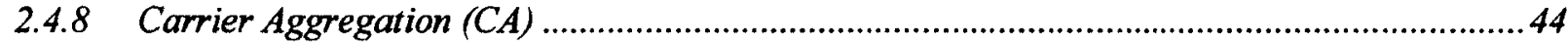

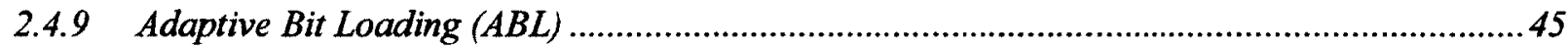

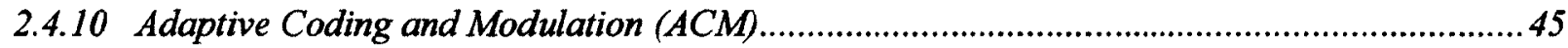

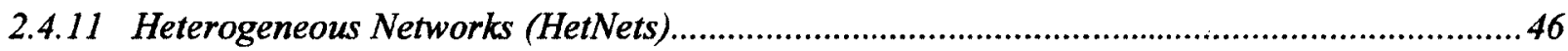

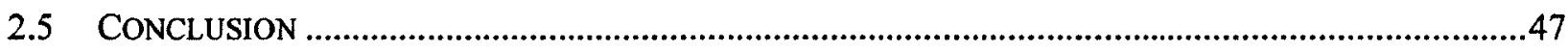

CHAPTER 3 . SPECTRUM SENSING TECHINIQUES.................................................................48

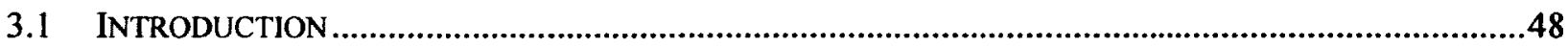

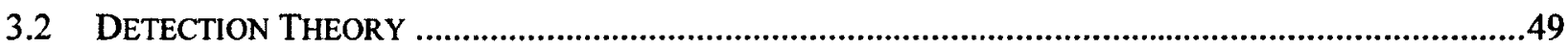

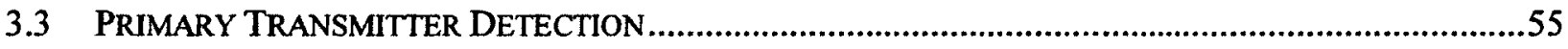

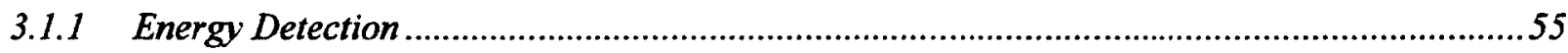

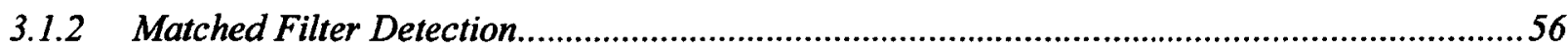

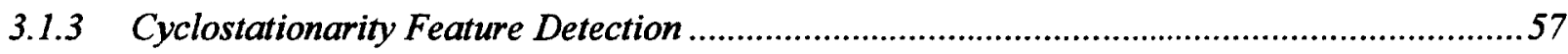

3.1.4 Waveform-Based/Autocorrelation-Based Sensing......................................................59

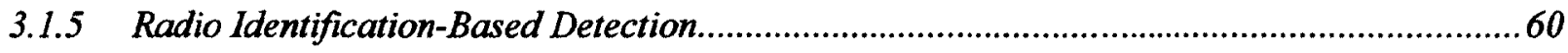

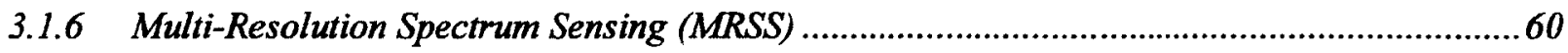




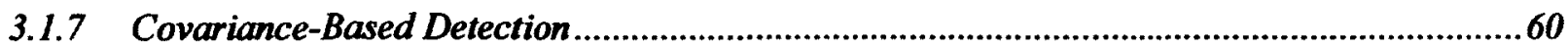

3.1.8 Multi-Taper Spectrum Sensing and Estimation (MTSE) .............................................60

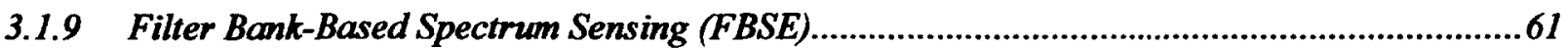

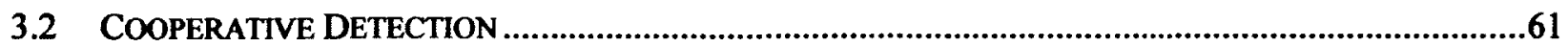

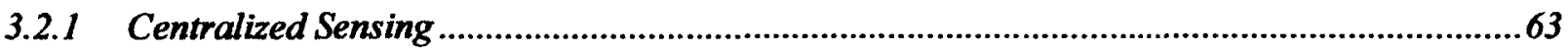

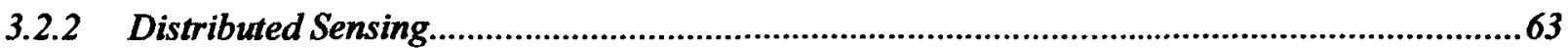

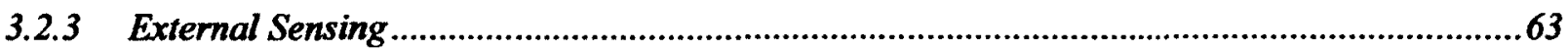

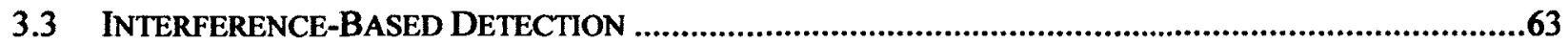

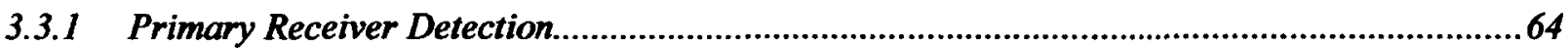

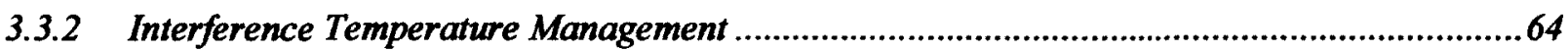

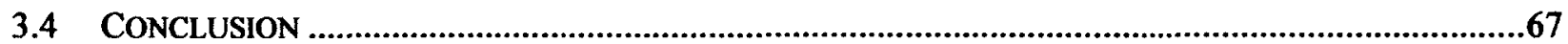

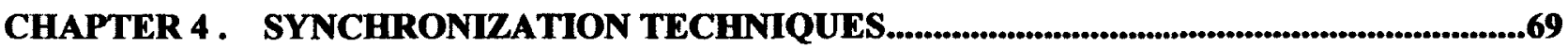

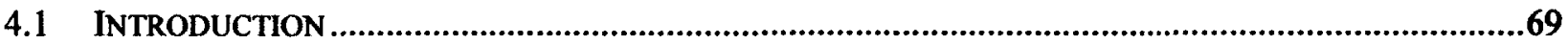

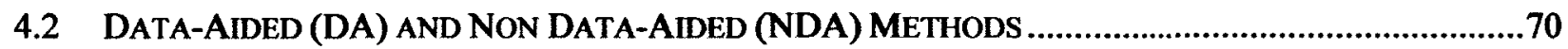

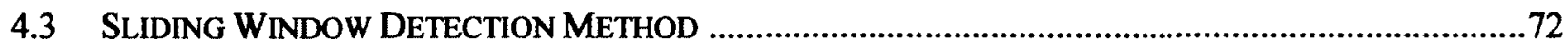

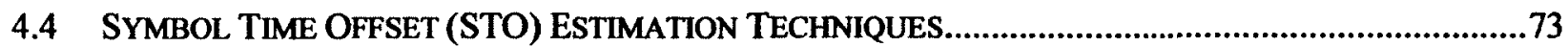

4.4.1 Non Data-Aided (NDA) Timing Offset Estimation .........................................................75

4.4.2 Data-Aided (DA) Timing Offset Estimation.......................................................................77

4.5 CARRIER FREQUENCY OFFSET (CFO) ESTIMATION TECHNIQUES ..............................................84

4.5.1 Non Data-Aided (NDA) Frequency Offset Estimation........................................................86

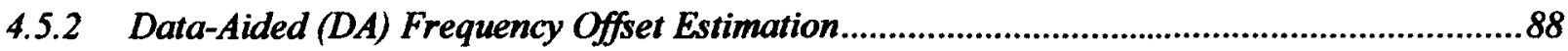

4.6 TIMING OFFSET AND FREQUENCY OFFSET COMPENSATION TECHNIQUES .................................92

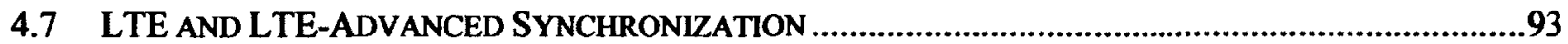

4.8 Modulation EFFICIENCY AND SYNCHRONIZATION PERFormanCE MEASUREMENT ................101

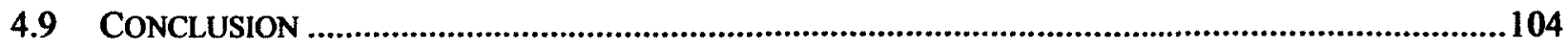




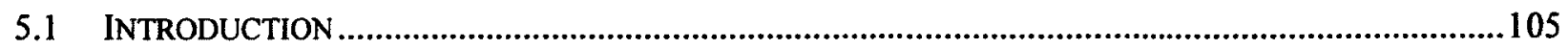

5.2 WAVEFORMS GENERATION, TRANSMISSION, AND RECEPTION SETUP ....................................... 105

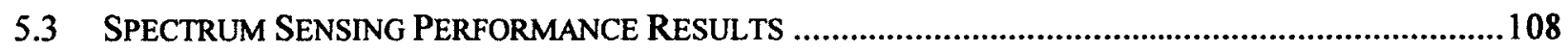

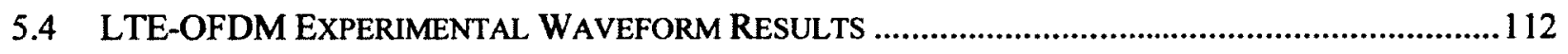

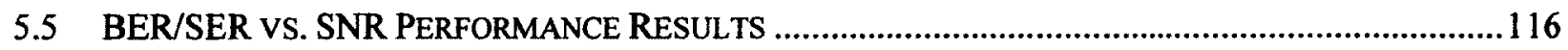

5.6 SYMBOL TIMING OFFSET ESTIMATION EXPERIMENTAL RESULTS ............................................ 122

5.7 CARRIER FREQUENCY OFFSET ESTIMATES EXPERIMENTAL RESULTS ...................................130

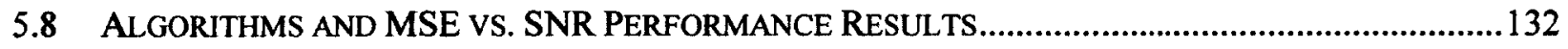

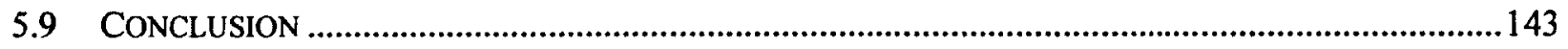

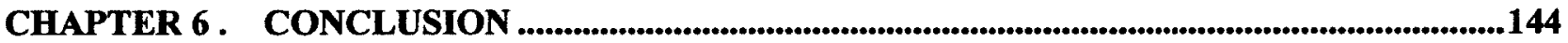

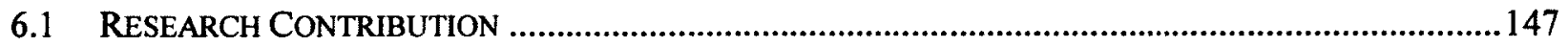

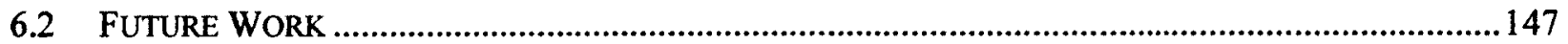

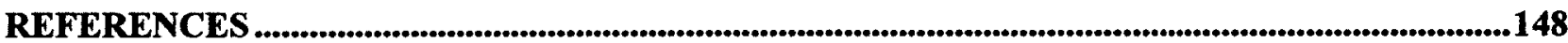

APPENDIX A. LTE-OFDM TRANSCEIVER SYSTEM SETUP .......................................................151

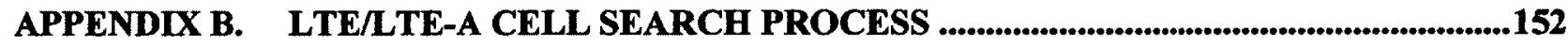

APPENDIX C. $\quad$ STO, CFO ESTIMATES, AND MSE DATA RESULTS....................................154 


\section{List of Figures}

Figure 2.1. 3GPPLTE and 4G LTE-Advanced Radio Frame Structure ...............................................5

Figure 2.2. LTE OFDM Resource Block (RB) with Normal Cyclic Prefix ..........................................5

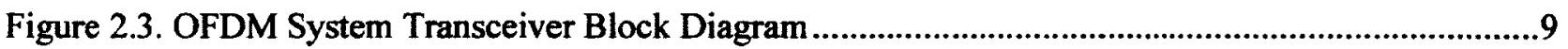

Figure 2.4. OFDM Symbol in Joint Time and Frequency-Domain ................................................. 10

Figure 2.5. Channel Impulse Response, ISI and Cyclic Prefix ......................................................

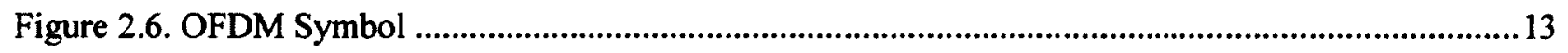

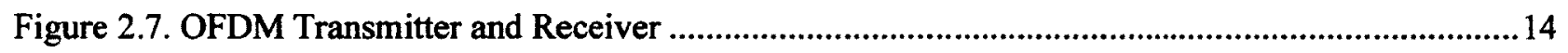

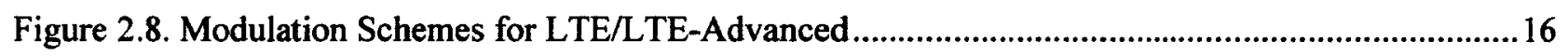

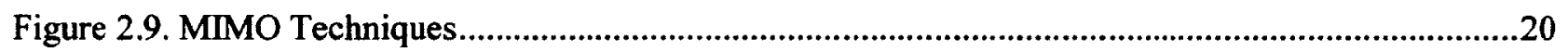

Figure 2.10. Time-Domain - Multipath-Induced Time Delay Results in ISI ....................................21

Figure 2.11. Frequency-Domain - Tx/Rx LO Mismatch/Motion-induced Doppler Shift CFO ICI ...........21

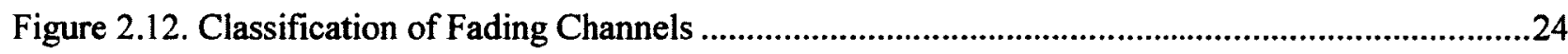

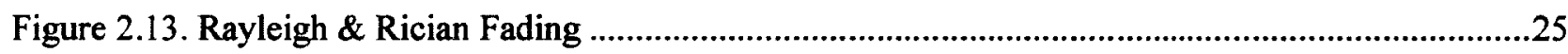

Figure 2.14. Flat Fading vs. Frequency-Selective Fading (Multipath Delay Spread)..............................26

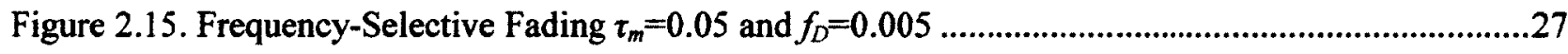

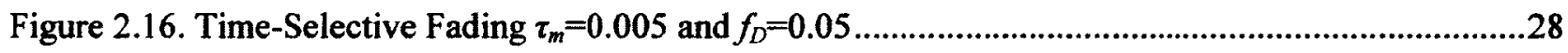

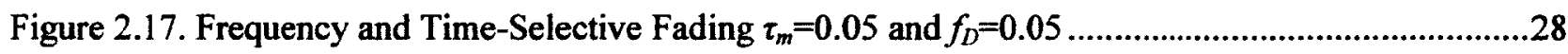

Figure 2.18. Slow Fading vs. Fast Fading (Doppler Spread) .............................................................29

Figure 2.19. Large-Scale Fading vs. Small-Scale Fading .....................................................................30

Figure 2.20. Power-Delay Profile vs. Doppler Power Spectrum..........................................................32

Figure 2.21. Time-Frequency Selectivity and Dispersion Duality .......................................................32

Figure 2.22. Time-Variant Channel Impulse Response and Doppler Functions......................................34

Figure 2.23. Frequency-Agile Transceiver Concept Block Diagram ....................................................40

Figure 2.24. Spectrum Hole and Dynamic Spectrum Access (DSA) ...............................................42 
Figure 2.25. LTE Adaptive Coding Modulation (ACM)

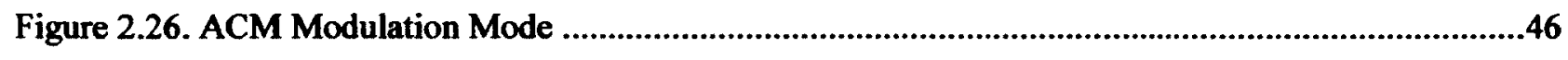

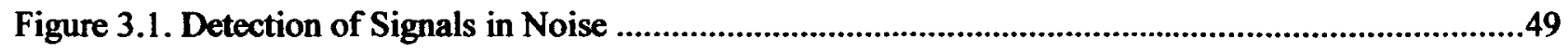

Figure 3.2. Probability of Occurrence (POC) $P_{D}$ and $P_{F A}$ Curves ................................................51

Figure 3.3. Receiver Operating Characteristics (ROC) $P_{D}$ and $P_{F A}$ Curves .........................................53

Figure 3.4. Sensing Performance SNR vs. Noise Power $P_{F A}$ and $P_{D}$ Trade-Off .................................54

Figure 3.5. Basic Primary Transmitter Detection Methods..........................................................55

Figure 3.6. Hidden Primary User Problem in Spectrum-Agile Radio...............................................62

Figure 3.7. Detecting Spectrum Opportunities: Spectrum Sensing Techniques ......................................65

Figure 3.8. Primary User (PU) Detection Complexity vs Accuracy Tradeoffs........................................66

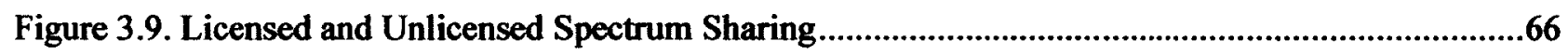

Figure 4.1. Data-Aided Sync Method using Training Sequence ( Matched Filter).................................70

Figure 4.2. Non-Data-Aided Synchronization Method using Cyclic Prefix (Correlator) .........................71

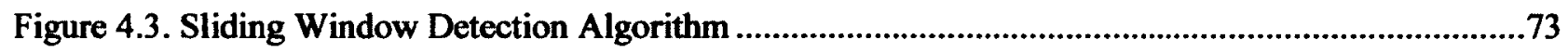

Figure 4.4. Scenarios of DFT Window Location Symbol Offset Synchronization.................................73

Figure 4.5. Block Diagram of Synchronizer using Cyclic Prefix...................................................75

Figure 4.6. Non Data-Aided (NDA) STO Estimation Technique using Cyclic Prefix .............................76

Figure 4.7. Time Synchronization based on Cyclic Prefix Correlation...............................................76

Figure 4.8. Timing Synchronization using Training Sequence ...........................................................78

Figure 4.9. Time Synchronizer using Training Symbol ...................................................................78

Figure 4.10. Time Synchronization using Identical Half Reference Symbol.........................................79

Figure 4.1 1. Data-Aided (DA) STO Estimation using Training Sequence Repetition ............................81

Figure 4.12. Fine Symbol Time Synchronization based on CIR Estimation .........................................83

Figure 4.13. Carrier Frequency Synchronization ..............................................................................84

Figure 4.14. Time and Frequency Synchronizer using Cyclic Prefix ....................................................86

Figure 4.15. Frequency Offset Estimation using Maximum Likelihood...................................................88 
Figure 4.16. Frequency Offset Estimation using Two OFDM Symbols .89

Figure 4.17. Data-Aided CFO Estimation using Pilot Tones [21] ....................................................90

Figure 4.18. OFDM Time and Frequency Synchronization Techniques Classification............................91

Figure 4.19. Proposed LTE/LTE-A OFDM Synchronization using Zadoff-Chu CAZAC ......................97

Figure 4.20. P-SCH and S-SCH Signals Location in LTE-OFDM Radio Frame ...................................98

Figure 4.21. Analysis of Zadoff-Chu CAZAC Matched Filtering LTE-OFDM Synchronization [34] ......99

Figure 4.22. Analysis of LTE-OFDM Time and Frequency Synchronization using CAZAC Sequence..100

Figure 4.23. BER vs. Eb/N0 Performance of LTE-OFDM Modulation over Wireless Channel ..............101

Figure 4.24. MSE vs. SNR Performance of LTE-OFDM Synchronization over Wireless Channel.........102

Figure 5.1. Digital Acquisition Hardware Receiver (R\&S FSV) Block Diagram...............................107

Figure 5.2. Receiver Operating Characteristics (ROC) $P_{D}$ vS. $P_{F A}$ Linear Scale...................................109

Figure 5.3. Receiver Operating Characteristics (ROC) $P_{D}$ vs. $P_{F A}$ Logarithmic Scale .........................109

Figure 5.4 Probability of Occurrence (POC) $\mathrm{P}_{\mathrm{D}}$ and $\mathrm{P}_{\mathrm{FA}}$ Tradeoff by Increasing the SNR ....................110

Figure 5.5. Probability of Occurrence (POC) $P_{D}$ and $P_{F A}$ Tradeoff by Decreasing the Noise Power .......111

Figure 5.6. LTE-OFDM DL BW=1.4MHz Power Spectrum Density (PSD) ....................................113

Figure 5.7. LTE-OFDM DL BW=3MHz Power Spectrum Density (PSD) ......................................113

Figure 5.8. LTE-OFDM DL BW=5MHz Power Spectrum Density (PSD) ......................................114

Figure 5.9. LTE-OFDM DL BW=10MHz Power Spectrum Density (PSD) .................................114

Figure 5.10. LTE-OFDM DL BW=15MHz Power Spectrum Density (PSD) .................................115

Figure 5.11. LTE-OFDM DL BW=20MHz Power Spectrum Density (PSD) ....................................115

Figure 5.12. Theoretical BER OFDM Coherent Demod LTE/LTE-A over AWGN Channel .................117

Figure 5.13. Theoretical SER OFDM Coherent Demod LTE/LTE-A over AWGN Channel .................117

Figure 5.14. Theoretical BER Coherent Demod LTE over Rayleigh Fading Chan ..............................118

Figure 5.15. Theoretical SER Coherent Demod LTE over Rayleigh Fading Chan ...............................118

Figure 5.16. Theoretical BER Coherent Demod LTE over Rician Fading Chan $(K=3)$........................120

Figure 5.17. Theoretical SER Coherent Demod LTE over Rician Fading Chan $(\mathrm{K}=3) \ldots . . . . . . . . . . . . . . . . . . . . .120$ 
Figure 5.18. Theoretical BER Coherent Demod LTE over Rician Fading Chan $(\mathrm{K}=6)$ 121

Figure 5.19. Theoretical SER Coherent Demod LTE over Rician Fading Chan $(K=6)$. 121

Figure 5.20. Symbol Time Offset (STO) Estimation 1.4 MHz, Downlink, CP=Normal 124

Figure 5.21. Symbol Time Offset (STO) Estimation 1.4 MHz, Downlink, CP=Extended 125

Figure 5.22. Symbol Time Offset (STO) Estimation $10 \mathrm{MHz}$, Downlink, $\mathrm{CP}=$ Normal 126

Figure 5.23. Symbol Time Offset (STO) Estimation $10 \mathrm{MHz}$, Downlink, CP=Extended 127

Figure 5.24. Symbol Time Offset (STO) Estimation $20 \mathrm{MHz}$ Downlink CP=Normal 128

Figure 5.25. Symbol Time Offset (STO) Estimation $20 \mathrm{MHz}$ Downlink CP=Extended 129

Figure 5.26. Experimental MSE vs. SNR 1.4MHz QPSK CFO $=0$ 133

Figure 5.27. Experimental MSE vs. SNR 3MHz 64-QAM CFO $=0$ 133

Figure 5.28. Experimental MSE vs. SNR 10MHz 64-QAM CFO $=0$ 134

Figure 5.29. Experimental MSE vs. SNR 10MHz 64-QAM CFO $=0$ 134

Figure 5.30. Experimental MSE vs. SNR 20MHz 64-QAM CFO $=0$ 135

Figure 5.31. Experimental MSE vs. SNR 20MHz 64-QAM CFO $=0$ 135

Figure 5.32. Experimental MSE vs. SNR 1.4MHz QPSK CFO $=0.25$ 136

Figure 5.33. Experimental MSE vs. SNR 3MHz 64-QAM CFO $=0.25$ 136

Figure 5.34. Experimental MSE vs. SNR 10MHz 64-QAM CFO $=0.25$ .137

Figure 5.35. Experimental MSE vs. SNR 10MHz 64-QAM CFO $=0.25$ 137

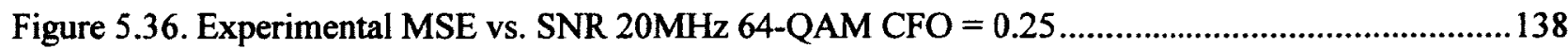

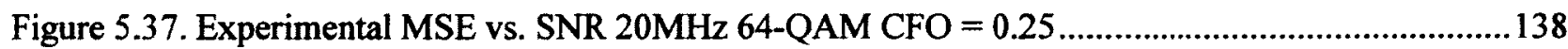

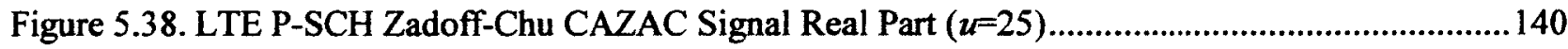

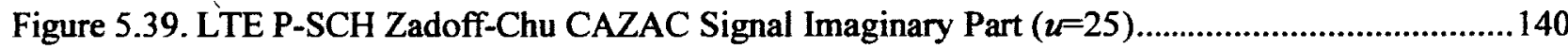

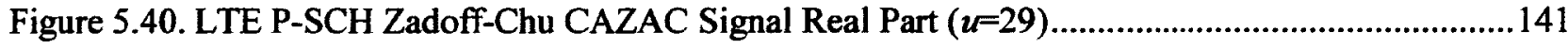

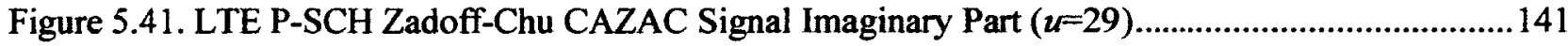

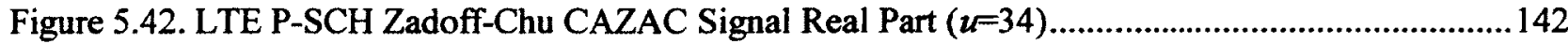

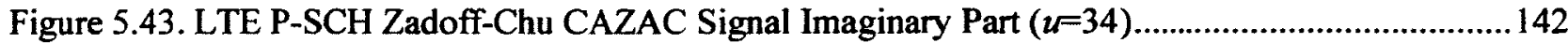




\section{List of Tables}

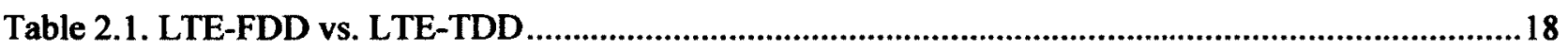

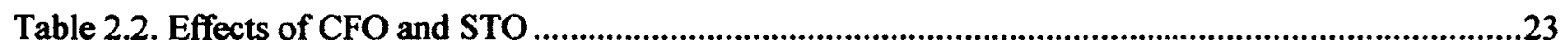

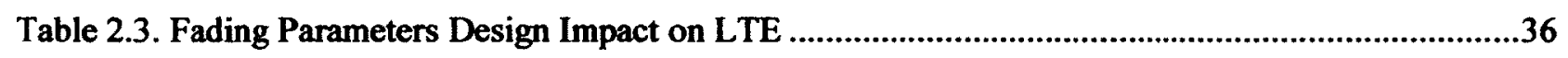

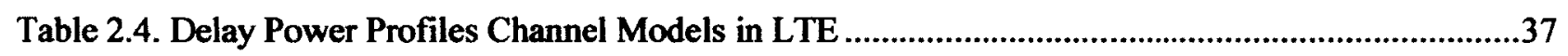

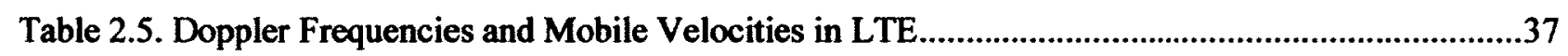

Table 3.1. Spectrum Sensing Receiver Sensitivity Requirements for CR.............................................57

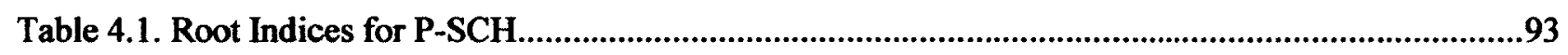

Table 5.1 LTE-OFDM Experimental I/Q Waveforms Measured Bandwidth and PSD .........................112 


\section{Acronyms}

\begin{tabular}{|c|c|}
\hline $\mathrm{ABL}$ & Adaptive Bit Loading \\
\hline $\mathrm{ACF}$ & Autocorrelation Function \\
\hline $\mathrm{ADC}$ & Analog to Digital Conversion \\
\hline $\mathrm{ACM}$ & Adaptive Coding Modulation \\
\hline ATPC & Automatic Transmitter Power Control \\
\hline BLUE & Best Linear Unbiased Estimation \\
\hline BPSK & Binary Phase Shift Keying \\
\hline $\mathrm{CA}$ & Carrier Aggregation \\
\hline CAF & Cyclic Autocorrelation Function \\
\hline CAS & Carrier Assignment Scheme \\
\hline CAZAC & Constant Amplitude Zero Autocorrelation \\
\hline $\mathrm{CBP}$ & Coexistence Beacon Protocol \\
\hline $\mathrm{CDF}$ & Cumulative Density Function \\
\hline CFAR & Constant False Alarm Rate \\
\hline CFO & Carrier Frequency Offset \\
\hline CIR & Channel Impulse Response \\
\hline $\mathrm{CP}$ & Cyclic Prefix \\
\hline $\mathrm{CR}$ & Cognitive Radio \\
\hline CRLB & Cramér-Rao Lower Bound \\
\hline CSI & Channel State Information \\
\hline CSS & Cooperative Spectrum Sensing \\
\hline CTF & Channel Transfer Function \\
\hline CTO & Coarse (Symbol) Time Offset \\
\hline
\end{tabular}




\begin{tabular}{|c|c|}
\hline DAB & Digital Audio Broadcasting \\
\hline DAC & Digital to Analog Conversion \\
\hline DFS & Dynamic Frequency Selection \\
\hline DFT & Discrete Fourier Transform \\
\hline $\mathrm{DL}$ & Downlink \\
\hline DSA & Dynamic Spectrum Access \\
\hline DSP & Digital Signal Processing \\
\hline DVB & Digital Video Broadcasting \\
\hline DwPTS & Downlink Pilot Time Slot \\
\hline FAR & Frequency-Agile Radio \\
\hline FCC & Federal Communications Commission \\
\hline FD & Frequency Division \\
\hline FD-LTE & Frequency Division Multiplex LTE \\
\hline FFO & Fractional (Carrier) Frequency Offset \\
\hline FFT & Fast Fourier Transform \\
\hline FTO & Fine (Symbol) Time Offset \\
\hline GEOLOC & Geolocation \\
\hline GP & Guard Period \\
\hline HetNet & Heterogeneous Network \\
\hline $\mathrm{ICl}$ & Inter Carrier Interference \\
\hline IFFT & Inverse Fast Fourier Transform \\
\hline IFO & Integral (Carrier) Frequency Offset \\
\hline IMS & IP Multimedia Subsystem \\
\hline ISI & Intersymbol Interference \\
\hline LRT & Likelihood Ratio Test \\
\hline LTE & Long Term Evolution \\
\hline
\end{tabular}




\begin{tabular}{|c|c|}
\hline LTE-A & LTE-Advanced \\
\hline MAC & Media Access Control \\
\hline MBMS & Multimedia Broadcast Multicast System \\
\hline MC & Multi Carrier \\
\hline MIH & Media Independent Handover \\
\hline MIMO & Multiple Input Multiple Output \\
\hline MLE & Maximum Likelihood Estimation \\
\hline MSE & Mean Squared Error \\
\hline MVUE & Minimum-Variance Unbiased Estimator \\
\hline OFDM & Orthogonal Frequency Division Multiplexing \\
\hline OSA & Opportunistic Spectrum Access \\
\hline PAPR & High Peak to Average Power Ratio \\
\hline PDF & Probability Density Function \\
\hline PHY & Physical Layer \\
\hline POC & Probability of Occurrence \\
\hline PRBS & Pseudorandom binary sequence \\
\hline PSAM & Pilot-Symbol Assisted Modulation \\
\hline P-SCH & Primary Synchronization Channel \\
\hline PSD & Power Spectrum Density \\
\hline PU & Primary User \\
\hline QAM & Quadrature Amplitude Modulation \\
\hline QPSK & Quadrature Phase Shift Keying \\
\hline RB & Resource Block \\
\hline RCF & Raised Cosine Filter \\
\hline RE & Resource Element \\
\hline RFO & Residual (Carrier) Frequency Offset \\
\hline
\end{tabular}




\begin{tabular}{|c|c|}
\hline ROC & Receiver Operating Characteristics \\
\hline SC & Single Carrier \\
\hline SC-FDMA & Single Carrier Frequency Division Multiple Access \\
\hline SCF & Spectral Correlation Function \\
\hline SDF & Spectral Density Function \\
\hline SDT & Signal Detection Theory \\
\hline SDR & Software-Defined Radio \\
\hline SM & Spatial Multiplexing \\
\hline SON & Self Organized Network \\
\hline S-SCH & Secondary Synchronization Channel \\
\hline STC & Space Time Coding \\
\hline STO & Symbol Time Offset \\
\hline SU & Secondary User \\
\hline UL & Uplink \\
\hline TD & Time Division \\
\hline TD-LTE & Time Division Multiplex LTE \\
\hline TPC & Transmit Power Control \\
\hline TVWS & TV White Space \\
\hline UE & User Equipment \\
\hline UpPTS & Uplink Pilot Time Slot \\
\hline VHO & Vertical Handover \\
\hline WSSUS & Wide-Sense Stationary Uncorrelated Scattering \\
\hline $\mathrm{XCF}$ & Cross-correlation Function \\
\hline $\mathrm{ZC}$ & Zadoff-Chu sequence \\
\hline
\end{tabular}




\section{List of Symbols}

$B_{\mathrm{C}} \quad$ Channel coherence bandwidth

$D_{\mathrm{C}} \quad$ Channel coherence distance

$T_{\mathrm{C}} \quad$ Channel coherence time

$f_{\mathrm{D}} \quad$ Doppler spread

$\tau_{R M S} \quad$ Channel delay spread (RMS)

$\tau_{\max } \quad$ Channel delay spread (maximum)

$\theta_{R M S} \quad$ Angular spread

$\mathrm{BW}_{\text {Chan }} \quad$ Channel bandwidth $\left(\mathrm{BW}_{\mathrm{Chan}}=N_{\mathrm{FFT}} \cdot \Delta f\right)$

$\mathrm{BW}_{\text {Trans }} \quad$ Channel bandwidth $\left(\mathrm{BW}_{\text {Trans }}=N_{\text {used }} \cdot \Delta f\right)$

$T_{\mathrm{CP}} \quad$ Cyclic prefix duration

$T_{\mathrm{SYM}} \quad$ Symbol time

$T_{\text {Useful }} \quad$ Useful symbol time

$T_{\mathrm{S}} \quad$ Sampling time

$\tau \quad$ Symbol time offset (STO)

$\varepsilon \quad$ Carrier frequency offset (CFO)

$\tau_{C} \quad$ Coarse Time Offset (CTO)

$\begin{array}{ll}\tau_{F} & \text { Fine Time Offset (FTO) }\end{array}$

$\varepsilon_{F} \quad$ Fine Frequency Offset (FFO)

$\varepsilon_{I} \quad$ Integral Frequency Offset (IFO)

$\varepsilon_{R} \quad$ Residual Frequency Offset (RFO

$\Delta f \quad$ Subcarrier frequency spacing

$N_{\mathrm{CP}} \quad$ Guard interval length

$N_{\text {FFT }} \quad$ FFT symbol length/Number of subcarriers

$N_{\text {used }} \quad$ Number of used subcarriers (not including DC subcarrier) 


\begin{tabular}{|c|c|}
\hline$N_{R B}^{D L / U L}$ & Number of resource blocks $\left(N_{R B}^{D L / U L}=N_{\text {usod }} / N_{s c}^{R B}\right)$ \\
\hline$N_{s c}^{R B}$ & Number of resource block per time slot \\
\hline$f_{c}$ & Carrier frequency \\
\hline$f_{S}$ & Sampling frequency \\
\hline$x(n)$ & Time-domain signal \\
\hline$h(n)$ & Channel impulse response (CIR) in time-domain \\
\hline$w(n)$ & Added White Gaussian Noise (AWGN) in time-domain \\
\hline$X(k)$ & Frequency-domain signal (Complex symbol Tx on the $k^{\text {th }}$ subcarrier) \\
\hline$\hat{X}(m)$ & Estimate of the complex modulation symbol $X(k)$ \\
\hline$H(k)$ & Channel Transfer Function (CTF) in frequency-domain \\
\hline$I(k)$ & Inter Carrier Interference (ICI) caused by CFO in frequency-domain \\
\hline$W(k)$ & Added White Gaussian Noise in frequency-domain \\
\hline$R_{x x^{*}}^{\alpha}$ & Cyclic Autocorrelation Function (CAF) \\
\hline$S_{x x^{*}}^{\alpha}$ & Spectral Density Function (SDF) \\
\hline$r_{x x}$ & Autocorrelation function (ACF) \\
\hline$r_{x y}$ & Cross-correlation function (XCF) \\
\hline$P_{F A}$ & Probability of false alarm \\
\hline$P_{C F A}$ & Probability of cooperative false alarm \\
\hline$P_{D}$ & Probability of detection \\
\hline$P_{C D}$ & Probability of cooperative detection \\
\hline$P_{M D}$ & Probability of misdetection \\
\hline$\Gamma$ & Gamma function \\
\hline$\chi^{2}$ & Chi-square distribution \\
\hline
\end{tabular}




\section{Chapter 1 Introduction}

OFDM systems operate by modulating a single high rate data stream onto multiple lower rate orthogonal subcarriers. The advantage of OFDM systems for high data rate communications is that the symbol length is relatively long, compared to the channel delay spread, thus reducing intersymbol interference (ISI). Most current OFDM systems use a cyclic prefix, where a portion of the end of each symbol is prepended to the symbol. A synchronization system can use this added redundancy (Non data-aided methods) to estimate the symbol time offset (STO) and carrier frequency offset (CFO) at the receiver. However, for multicarrier transmission in frequency-selective fading channel environment the frequency synchronization has to be assisted by the transmission of known training sequences (Data-aided methods). Since each subcarrier is flat fading, pilot-symbol assisted modulation (PSAM) techniques [11] from single-carrier flat fading systems are directly applicable to OFDM.

\subsection{Motivation}

Two major challenges arise when an OFDM signal is transmitted over a dispersive channel. First, the channel dispersion destroys the orthogonality between subcarriers and causes intercarrier interference (ICI). Second, a system may transmit multiple OFDM symbols in a series so that a dispersive channel causes intersymbol interference (ISI) between successive OFDM symbols. Multi-carrier OFDM systems have unique implementation challenges that are not present in single-carrier systems. Single-carrier (SC) is more sensitive to symbol timing errors and less sensitive to frequency offsets, whereas multi-carrier OFDM is more resilient to timing errors in estimating the start of a symbol than single carrier system (this is due to the OFDM longer symbol period and its cyclic prefix), yet highly sensitive to frequency offsets and phase noise in the receiver RF and sampling clock oscillators (this is due to the narrowness of the OFDM subcarrier). Multi-carrier is more sensitive to Doppler spreads than signal-carrier modulated systems. Thus synchronization in both time and frequency-domain is a critical and crucial problem in OFDM systems since accurate frequency and time synchronization is required. 


\subsection{Objectives}

This research thesis investigates several state-of-the-art spectrum sensing methods and the latest research on synchronization techniques for frequency-agile radios from the LTE and OFDM perspective.

There are four major research contributions made by this thesis. The first contribution is a comprehensive study, investigation, and classification of the latest research on spectrum sensing mechanisms in the spectrum-agile radio context. The second contribution is an experimental study of LTE-OFDM time and frequency synchronization techniques. The third contribution is a proposed OFDM synchronization algorithm based on the Zadoff-Chu CAZAC pilot sequence (i.e. the LTE primary synchronization signal) based cross-correlation. The novelty here is an application of a known technique by Classen \& Meyr 94 [21] to a new area of LTE/LTE-Advanced. The fourth contribution is the development and evaluation of a proof-of-concept apparatus. That is, in order to replicate the LTE-OFDM realm of waveform creation, transmission, and reception we have used a combination of four very sophisticated and specialized software packages and hardware instruments: IQCreator ${ }^{\mathrm{TM}}$ (for LTE-OFDM waveform creation), Aeroflex IFR 3416 (for RF transmission), R\&S FSV (for BB reception), and CRC Spectrum Explorer (for I/Q BB waveform capture).

\subsection{Thesis Organization}

Chapter 2 lays the foundation background theory for the rest of the thesis. Specifically, Chapter 2 discusses the background on time-selective fading and frequency-selective fading aspects of LTE and OFDM. Chapter 3 analyzes the main categories of spectrum sensing techniques. Chapter 4 studies synchronization techniques, focusing on the symbol time offset and carrier frequency offset in the timedomain and frequency-domain. Chapter 5 performs an experimental demonstration and BER performance of synchronization algorithms to estimate the STO and CFO based on LTE-OFDM VQ signal waveforms. Finally, chapter 6 provides the conclusion and recommendations for future research directions. 


\section{Chapter 2 . Background Theory}

\subsection{Introduction}

The major emphasis of this chapter is a background review of LTE (OFDM and MIMO), interferences and uncertainties in OFDM, and the radio channel characterization. The concept of a spectrum-agile radio is also introduced and discussed. A frequency-agile radio is a secondary user that dynamically accesses available spectrum in an unlicensed manner when that spectrum is unoccupied by a primary user.

One of the main reasons to use OFDM is to increase robustness against frequency selective fading. In a fundamental contribution to OFDM, Robert W. Chang [1] from Bell Labs, has first developed in 1966 general conditions for the shapes of pulses, defined as the combination of transmitter filters and channel characteristics, with bandlimited and overlapping spectra. The cyclic prefix was first proposed by Peled and Ruiz from IBM Watson Research Center [2] in 1980. An OFDM symbol has a useful period $T$ and preceding each symbol is a cyclic prefix of length $T_{\mathrm{g}}$, which is longer than the channel impulse response (CIR) so that there will be no inter-symbol interference (ISI). Cyclic prefix accommodates the decaying transient of the previous symbol and prevents the initial transient reaching the current symbol.

In 2009, the ITU-R organization specified the IMT-Advanced (International Mobile Telecommunications Advanced) requirements for $4 \mathrm{G}$ standards, setting peak speed requirements for $4 \mathrm{G}$ service at $100 \mathrm{Mbps}$ for high mobility communication (such as from trains and cars) and 1 Gbps for low mobility communication (such as pedestrians and stationary users). A $4 \mathrm{G}$ system is expected to provide a comprehensive and secure all-IP based mobile broadband solution to laptop computer wireless modems, smartphones, and other mobile devices. Facilities such as ultra-broadband Internet access, IP telephony, gaming services, and streamed multimedia may be provided to users. 


\subsection{Long Term Evolution (LTE) Fundamentals}

The Long Term Evolution (LTE) project was initiated in 2004. The flexible scalable channel bandwidth is a key feature of LTE, specified at $1.4,3,5,10,15$, and $20 \mathrm{MHz}$ in both uplink and downlink. This allows LTE to be flexibly deployed where other systems exist today. LTE is based on the OFDM modulation techniques and adopts single-carrier frequency division multiple access (SC-FDMA) on the uplink transmission and OFDMA on the downlink transmission. OFDMA means different users on different subcarriers. The key requirement that sets $4 \mathrm{G}$ apart from previous standards is enhanced peak data rates that reach as high as $1 \mathrm{Gbps}$ for low mobility applications and $100 \mathrm{Mbps}$ for high mobility (compared to $3 \mathrm{G}$ peak rate of $2 \mathrm{Mbps}$ for indoor low mobility applications and $144 \mathrm{kbps}$ for vehicular applications). In LTE-Advanced, the only way to achieve significantly higher data rates is to increase the channel bandwidth, where the upper limit bandwidth is at $100 \mathrm{MHz}$ with $40 \mathrm{MHz}$ the expected for minimum performance. Only two $4 \mathrm{G}$ candidates are being actively developed today: LTE-Advanced (an all-IP architecture unlike the mixed circuit and packet in LTE) and IEEE $802.16 \mathrm{~m}$ (which is the evolution of the WiMAX standard known as Mobile WiMAX).

In LTE, the length of a radio frame is $10 \mathrm{~ms}$ long and consists of 10 sub-frames of $1 \mathrm{~ms}$ each as shown in Figure 2.1 [33]. One sub-frame results in the duration of $1 \mathrm{~ms}$ and is built of two slots. A slot has a fixed length of $0.5 \mathrm{~ms}$ and includes either seven OFDM symbols with a normal cyclic prefix length or six OFDM symbols with an extended cyclic prefix length. The OFDM symbols with a normal cyclic prefix have a cyclic prefix length of $T_{c p}=5.2 \mu \mathrm{s}$ for the first OFDM symbol and of $T_{c p}=4.7 \mu \mathrm{s}$ for the remaining OFDM symbols. In the case of the extended cyclic prefix, the length is $T c p=16.67 \mu$. The extended cyclic prefix is used in scenarios with a long delay spread and for multicast and broadcast services in the single-frequency network mode. The OFDM symbol duration without a guard interval is always $66.7 \mu$ s. A resource block (RB) has a duration of one slot and consists of 12 adjacent sub-carriers $\left(N_{s c}^{R B}=12\right)$ with a subcarrier frequency spacing of $\Delta f=15 \mathrm{kHz}$. 


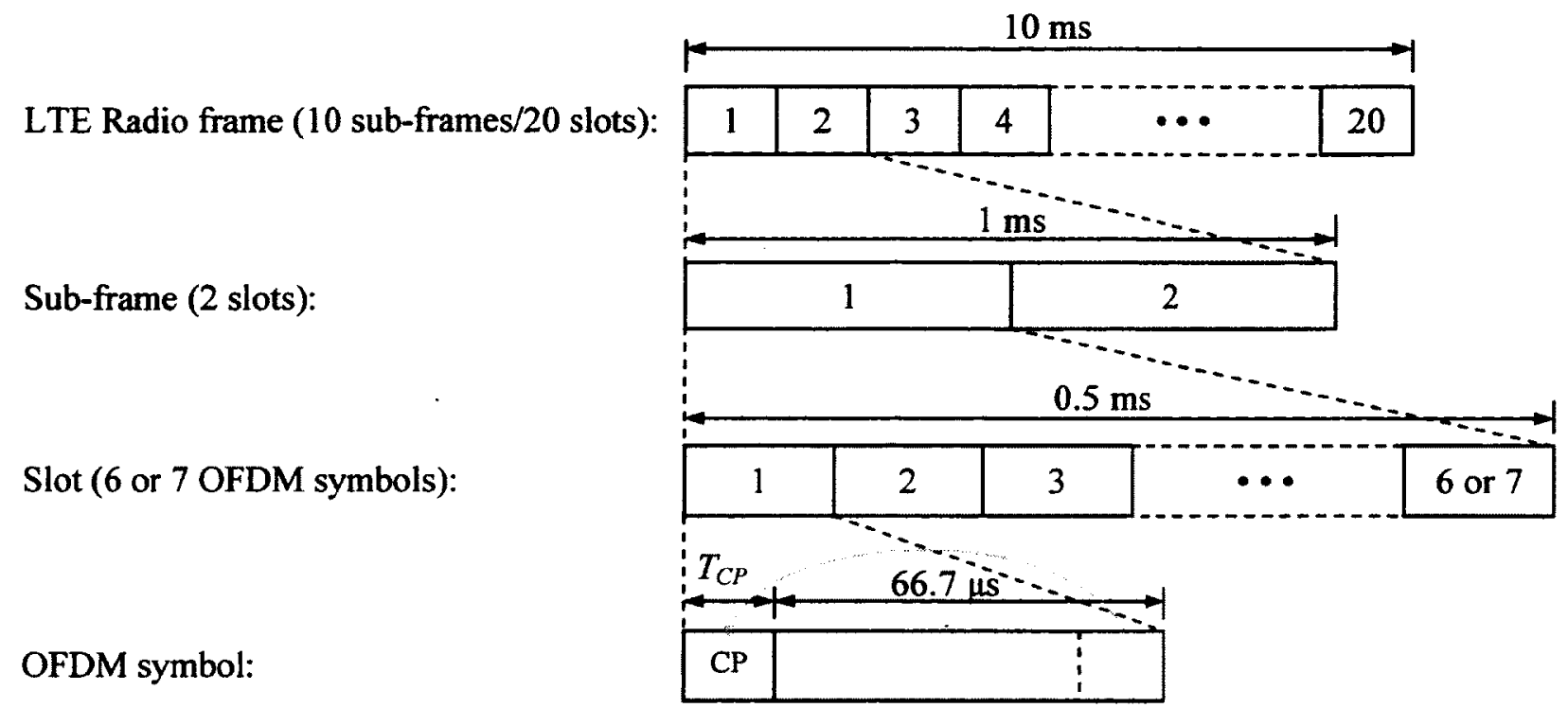

Figure 2.1. 3GPPLTE and 4G LTE-Advanced Radio Frame Structure

Figure 2.2 shows a resource block (RB) that has the duration of one slot and consists of 12 adjacent subcarriers [33]. Thus, for the normal cyclic prefix length, $84(12 \times 7)$ resource elements $(\mathrm{REs})$ are transmitted in one resource block while in the case of the extended cyclic prefix, $72(12 \times 6)$ resource elements are transmitted. A RB corresponds to a symbol transmitted on one sub-carrier in one OFDM symbol. The sub-carrier distance in the downlink is $15 \mathrm{kHz}$ so that a RB has a total bandwidth of $180 \mathrm{kHz}$.

1 resource block $N_{s c}^{R B} \cdot N_{s y m b}^{D L / U L}$

(12 sub-carriers $\mathrm{x} 7$ OFDM symbols)

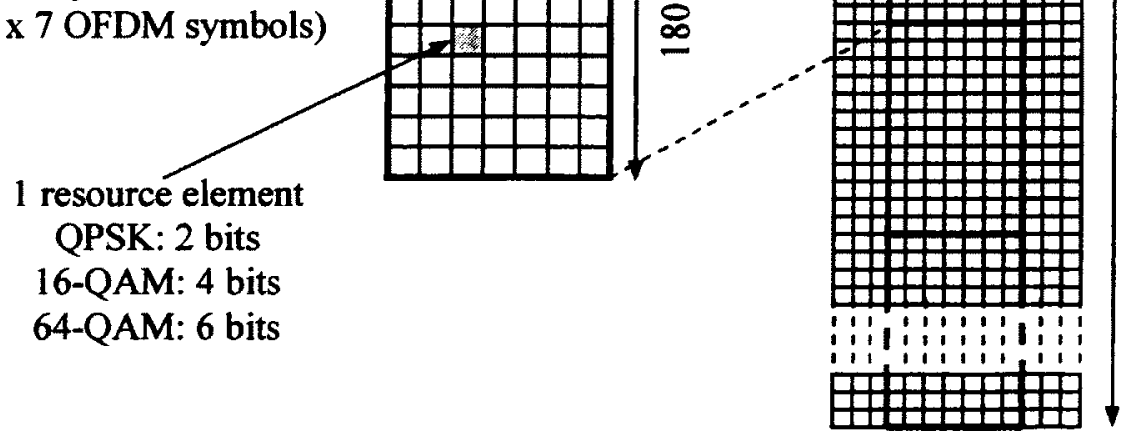

Figure 2.2. LTE OFDM Resource Block (RB) with Normal Cyclic Prefix 
In order to achieve these requirements, LTE has enabled two main key technologies: OFDM and MIMO in which we will discuss in more details in the next two sections.

\subsubsection{Orthogonal Frequency Division Multiplexing (OFDM)}

OFDM subcarriers have a $\sin c(\sin (x) / x)$ frequency response resulting in overlap in the frequency domain. This overlap however does not cause any interference due to the orthogonality of the subcarriers. The OFDM receiver uses a time and frequency synchronized FFT to convert the OFDM time waveform back into the frequency domain. In this process the FFT picks up discrete frequency samples, corresponding to just the peaks of the carriers. At these frequencies, all other carriers pass through zero amplitude eliminating any interference between the subcarriers.

The advantages of OFDM are: high spectral efficiency (efficient bandwidth usage), simplified frequencydomain equalization (over single-carrier with time-domain equalization), resistance (robust) to Frequency-selective fading (caused by multipath delay spread), scalability for different bandwidth (LTE BW: 1.4, 3, 5, 10, 15, 20MHz), duplex flexibility (TDD/Unpaired spectrum, FDD/Paired spectrum, Halfduplex FDD), and advantageous uses of revolutionary antenna diversity techniques (MIMO, beamforming, etc.). However, the disadvantages of OFDM are: high peak to average power ratio (PAPR), high sensitivity to carrier frequency offset (CFO), sensitivity to Time-selective fading (caused by motion Doppler spread), sensitivity to phase noise, complexity (synchronization problems, IFFT/FFT for modulation/demodulation), and performance overhead due to the cyclic prefix and pilot tones.

An OFDM transmitter maps the message bits into a sequence of PSK or QAM symbols which will be converted into $N$ parallel streams. Let $x(t)$ the continuous-time OFDM transmitted signal:

where $\Delta f$ is the frequency spacing.

$$
x(t)=\sum_{k=0}^{N-1} X(k) e^{j 2 \pi\left(f_{c}+k \Delta f\right) t}
$$


Then the $N$-point inverse discrete Fourier transform (IDFT) of M-PSK or M-QAM data symbols $X[k]$ can be computed efficiently using the IFFT algorithm.

$$
x[n]=\frac{1}{\sqrt{N}} \sum_{k=0}^{N-1} X[k] e^{j \frac{2 \pi k n}{N}} \quad n=0, \ldots, N-1
$$

where $N$ is the number of subcarriers or the IFFT size, $k$ is the subcarrier index, $n$ is the time index, and $1 / N$ is the normalized frequency separation of the subcarriers. Note that $x[n]$ and $X[k]$ which denote the transmitted symbol at the $k$ subcarrier, form an $N$-point discrete Fourier transform (DFT) pair, whose relationship can be expressed as:

$$
X[k]=\sum_{n=0}^{N-1} x[n] e^{-j \frac{2 \pi k n}{N}} \quad k=0, \ldots, N-1
$$

Let $y[n]$ be the received discrete-time signal after passing through the channel.

$$
y[n]=h[n] \cdot x[n]+w[n]
$$

where $x[n], y[n], h[n]$, and $w[n]$ denote the $n^{\text {th }}$ time index sequence of the transmitted signal, received signal, channel impulse response, and noise in the time-domain respectively.

Let $Y[k]$ be the received symbol at the $k^{\text {th }}$ subcarrier. Then the $N$-point DFT of $y[n]$ can be computed efficiently by using the FFT algorithm.

$$
Y[k]=\sum_{n=0}^{N-1} y[n] e^{-j \frac{2 \pi k n}{N}}=H[k] X[k]+W[n] \quad k=0, \ldots, N-1
$$

where $X[k], Y[k], H[k]$, and $W[k]$ denote the $k^{\text {th }}$ subcarrier frequency component of the transmitted symbol, received symbol, channel transfer function, and noise in the frequency-domain respectively. Since $Y[k]=H[k] \cdot X[k]$ under no noise condition, the transmitted symbol can be detected with a one-tap equalization, which simply divides the received symbol by the channel (i.e., $X[k]=Y[k] / H[k]$ ). It is noted that $Y[k] \neq H[k] \cdot X[k]$ without a CP, since DFT $\{y[n]\} \neq \operatorname{DFT}\{x[n]\} \cdot \operatorname{DFT}\{h[n]\}$ when $y[n]=x[n] * h[n]$, where * represents the convolution operation. In fact $Y[k]=H[k] \cdot X[k]$ when $y[n]=x[n] \otimes h[n]$, where $\otimes$ 
denotes the operation of circular convolution. In other words, insertion of a CP in the transmitter makes the transmit samples circularly-convolved with the channel samples, which yields $Y[k]=H[k] \cdot X[k]$ as desired in the receiver. The multiplication of the DFTs of two sequences in the frequency-domain is equivalent to the circular convolution of the two sequences in the time-domain.

$$
x_{1}(n) \otimes x_{2}(n) \stackrel{D F T}{\Longleftrightarrow} X_{1}(k) X_{2}(k)
$$

Let $x_{1}[n]$ and $x_{2}[n]$ be two finite-duration sequences of length $N$. Their respective N-point DFTs are:

$$
X_{1}[k]=\sum_{n=0}^{N-1} x_{1}(n) e^{-\left(\frac{j 2 \pi k n}{N}\right)} ; \quad X_{2}[k]=\sum_{n=0}^{N-1} x_{2}(n) e^{-\left(\frac{j 2 \pi k n}{N}\right)} \quad k=0,1, \ldots, N-1
$$

If we multiply the two DFTs together, the result is a DFT, say $X_{3}[k]$, of a sequence $x_{3}[n]$ of length $N$ :

$$
X_{3}[k]=X_{1}[k] \cdot X_{2}[k] \quad k=0,1, \ldots, N-1
$$

The IDFT of $\left\{X_{3}[k]\right\}$ is called the circular convolution:

$$
x_{3}[m]=x_{1}[n] \otimes x_{2}[n]=\sum_{n=0}^{N-1} x_{1}(n)\left(x_{2}(m-n)\right)_{N} \quad m=0,1, \ldots, N-1
$$

The finite-length channel impulse response is defined as:

$$
h[n]=\sum_{k=0}^{M-1} h_{k} \delta[n-k]
$$

where $M$ : length of the CIR; $k$ : coefficients are assumed to be time-invariant and known to the receiver.

Figure 2.3 shows a typical LTE transceiver system illustrating the location of time and frequency synchronization as well as channel estimation block. As shown, the synchronization block can be performed in time-domain (pre-FFT) or frequency-domain (post-FFT). The channel estimation block can also be performed using the time-domain approach or frequency-domain approach. In the time-domain approach, the channel impulse responșe (CIR) is estimated before FFT processing of the training symbols. In the frequency-domain approach, the channel frequency response (CFR) is estimated by using two repeat training symbols. 

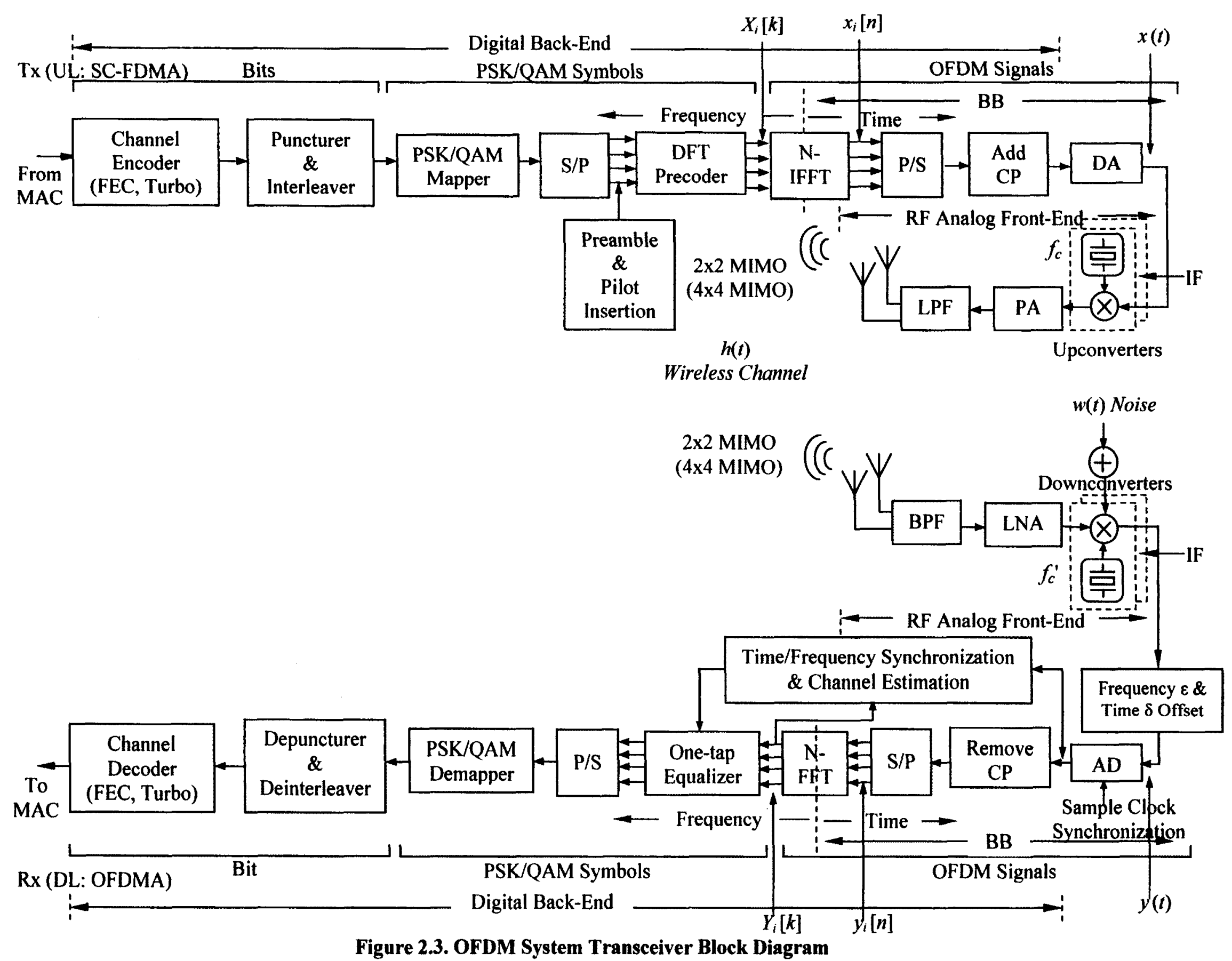

Figure 2.3. OFDM System Transceiver Block Diagram 
The OFDM guard interval can be inserted in two different ways. One is the zero padding (ZP) that pads the guard interval with zeros. The other is the cyclic extension of the OFDM symbol with CP (cyclic prefix) or CS (cyclic suffix). CP extends the OFDM symbol by copying the last samples of the OFDM symbol into its front. This is shown in Figure 2.4, illustrating jointly in the time and frequency domains.

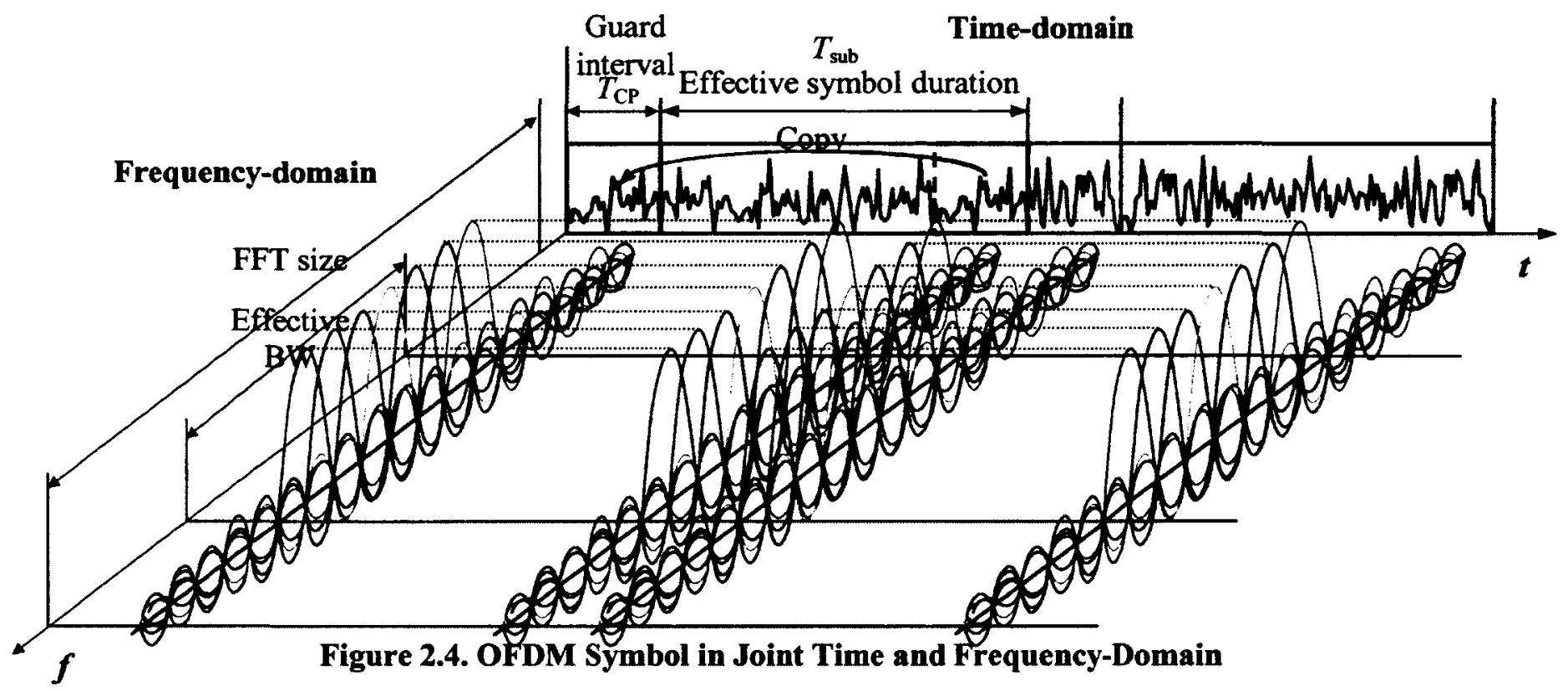

The baseband signal within an OFDM symbol can be written as:

$$
x(t)=\sum_{k=0}^{N-1} X(k) \cdot e^{j 2 \pi k \Delta f t}
$$

where $N$ is the number of subcarriers, $X(k)$ is the complex modulation symbol transmitted on the $k^{\text {th }}$ subcarrier $e^{2 \pi} \Delta^{f}$, and $\Delta f$ is the subcarrier spacing.

At the receiver, the estimate $\hat{X}(m)$ of the complex modulation symbol $X(k)$ is obtained by multiplying the received signal with $e^{-j 2 \pi k \Delta f t}$ and integrating over an OFDM symbol duration:

$$
y(t)=\hat{X}(m)=\int_{t=0}^{T_{S}}[x(t)+w(t)] \cdot e^{-j 2 \pi m \Delta f t} d t
$$


where $T_{\mathrm{S}}$ is the OFDM symbol duration, $x(t)$ is the transmitted signal, $w(t)$ is the AWGN noise, and $\Delta f$ is the subcarrier spacing.

The mathematics given below as background will become important in Chapter 4 . Let $x(n)$ the discrete-time signal and $X(\omega)$ its Fourier transform. By definition the Fourier transform is given by:

$$
X(\omega)=\sum_{n=-\infty}^{+\infty} x(n) e^{-j \omega n} \quad 0 \leq \omega \leq 2 \pi
$$

The inverse FT (IFT):

$$
x(n)=\frac{1}{2 \pi} \int_{2 \pi} X(\omega) e^{j \omega n} d \omega \quad 0 \leq \omega \leq 2 \pi
$$

A linear Time-Shift

$$
x(n-k) \stackrel{F T}{\longleftrightarrow} X(\omega) e^{-j \omega k}
$$

A Linear Frequency-Shift

$$
e^{j \omega_{0} n} x(n) \stackrel{F T}{\longleftrightarrow} X\left(\omega-\omega_{0}\right)
$$

The linear convolution

$$
x_{1}(n) * x_{2}(n) \stackrel{F T}{\longleftrightarrow} X_{1}(\omega) X_{2}(\omega)
$$

Let $x(n)$ be the discrete-time signal and $X(k)$ its discrete Fourier transform (sample sequence). Then we now have:

The Discrete-Fourier Transform (DFT):

$$
X(k)=\sum_{n=0}^{N-1} x(n) e^{-j 2 \pi k n / N} \quad k=0,1,2, \ldots, N-1
$$

The Inverse DFT (IDFT):

$$
x(n)=\frac{1}{N} \sum_{k=0}^{N-1} X(k) e^{j 2 \pi k n / N} \quad n=0,1,2, \ldots, N-1
$$


A Circular Time-Shift

$$
x((n-l))_{\mathrm{N}} \stackrel{\text { DFT }}{\stackrel{N}{\longrightarrow}} X(k) e^{-j 2 \pi k l / N}
$$

A Circular Frequency-Shift

$$
e^{j 2 \pi \ln / N} x(n) \stackrel{\text { DFT }}{\longrightarrow} X((k-l))_{N}
$$

The Circular Convolution

$$
x_{1}(n) \otimes x_{2}(n) \stackrel{D F T}{N} X_{1}(k) X_{2}(k)
$$

The previous mathematic formulas are very important background to pave the way for OFDM synchronization techniques that will be discussed later in chapter 4 .

Although the receiver is typically configured to discard the cyclic prefix (CP) samples, the cyclic prefix serves two important purposes: as a guard interval, it eliminates the inter-symbol interference from the previous symbol as shown in Figure 2.5 below, and it converts linear convolution into circular convolution. As a repetition of the end of the symbol, it allows the linear convolution of a frequency-selective multipath channel to be modeled as circular convolution, which in turn may be transformed to the frequency domain using a discrete Fourier transform using simple frequencydomain processing.
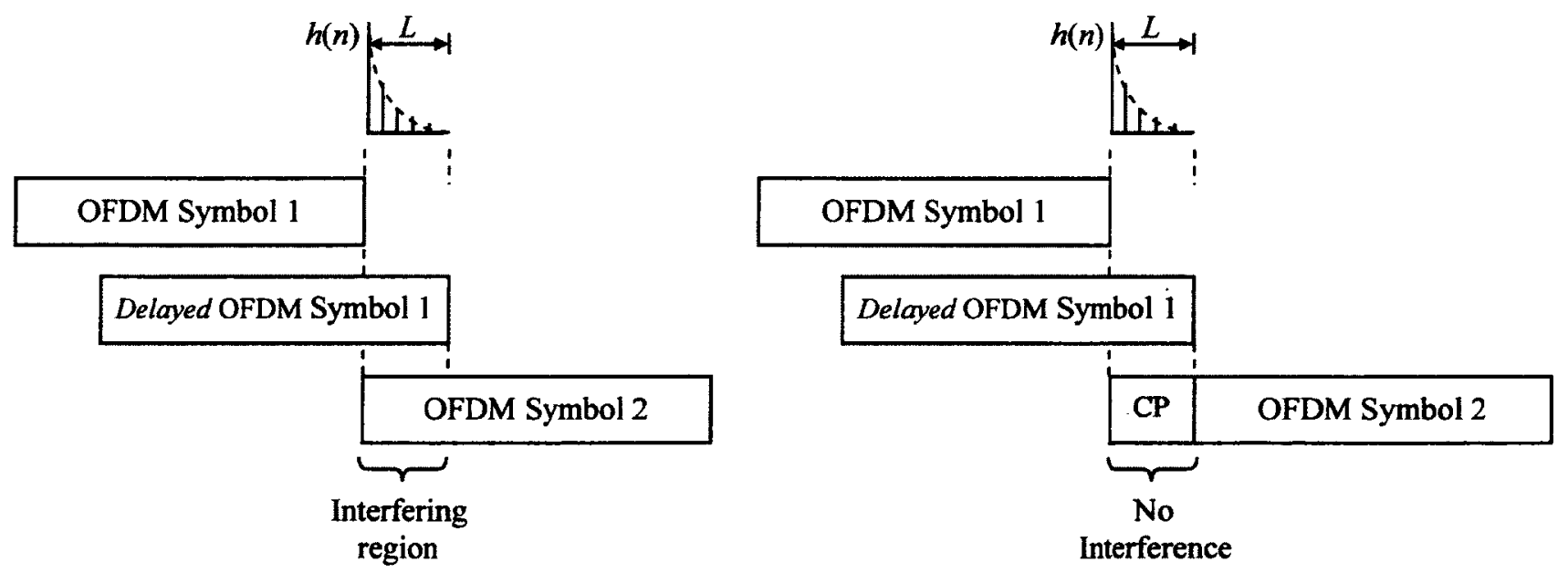

Figure 2.5. Channel Impulse Response, ISI and Cyclic Prefix 


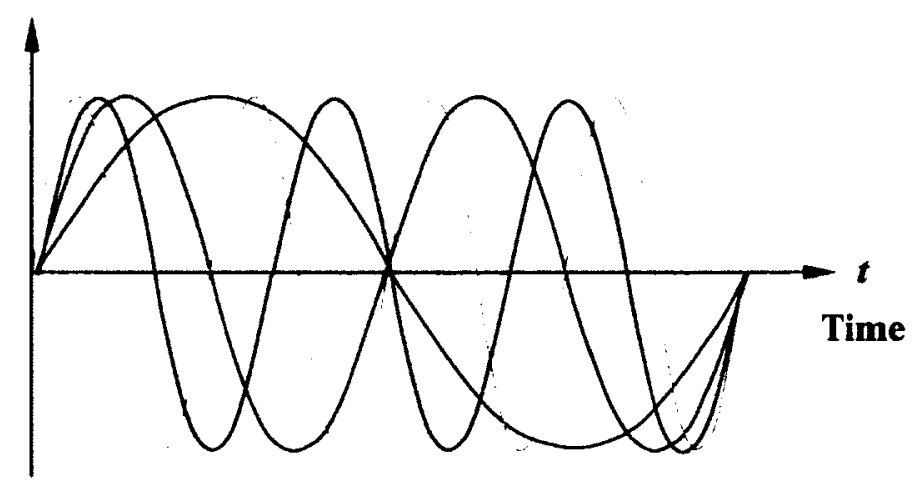

a) OFDM Symbol (time-domain)

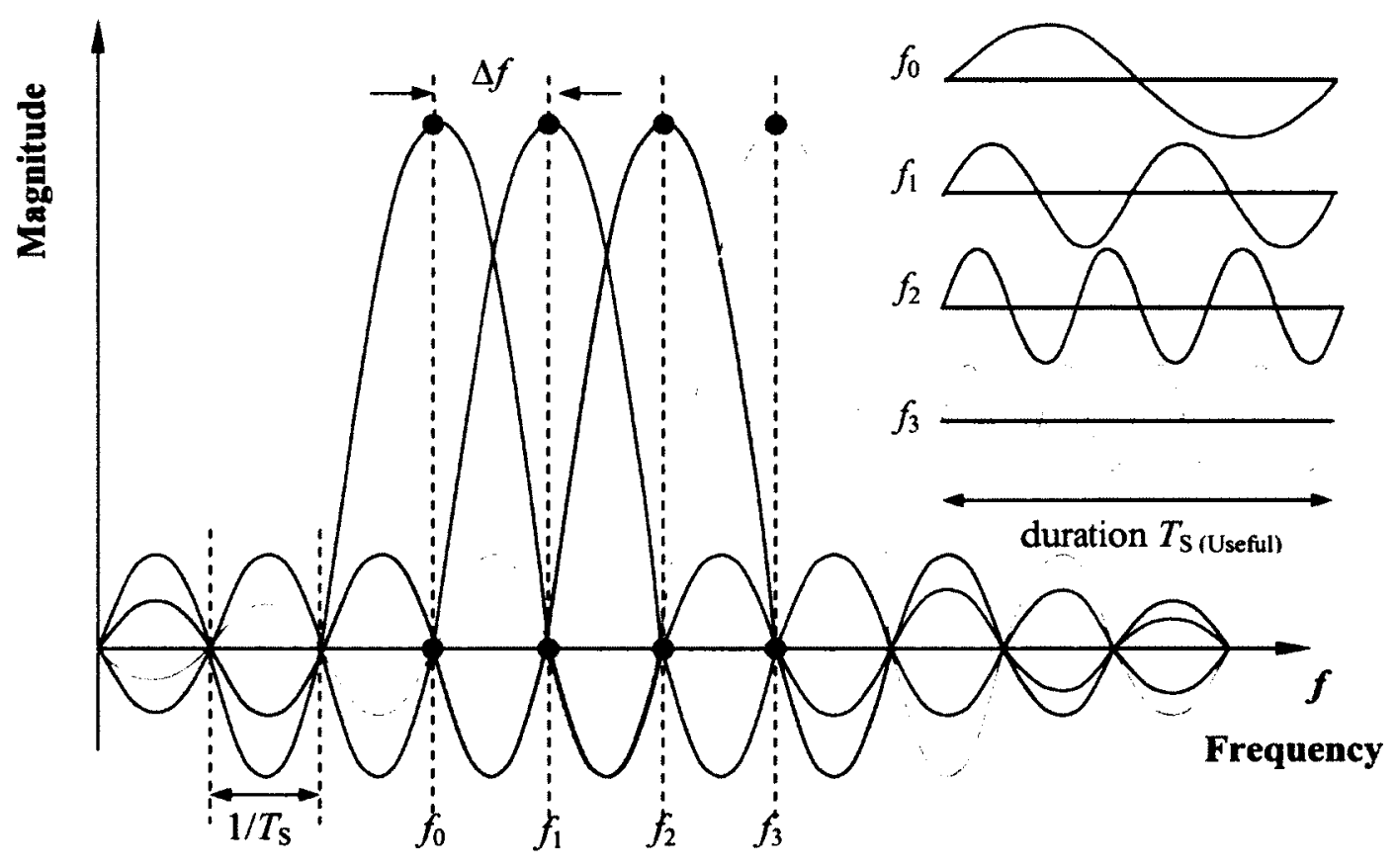

b) OFDM Symbol (frequency-domain)

Figure 2.6. OFDM Symbol

Figure 2.6 (a) illustrates OFDM signals in the time-domain and Figure 2.6 (b) shows OFDM spectra in the frequency-domain. The main idea is to maintain or preserve orthogonality among subcarrier components by eliminating the inter-symbol interference (ISI) from the previous symbol and furthermore, inter-channel interference (ICI). 
The heart of an OFDM modulator and demodulator are the inverse FFT (IFFT) and FFT respectively, as shown in Figure 2.7. The employment of discrete Fourier transform to replace the banks of sinusoidal signal generators at the transmitter and the demodulator significantly reduces the implementation complexity of OFDM modems. Modulation is the "piggy-backing" of a signal containing information onto another signal, called a carrier, which usually has a constant, and much higher, frequency. Demodulation is the process of removing the carrier signal to obtain the original information signal waveform.
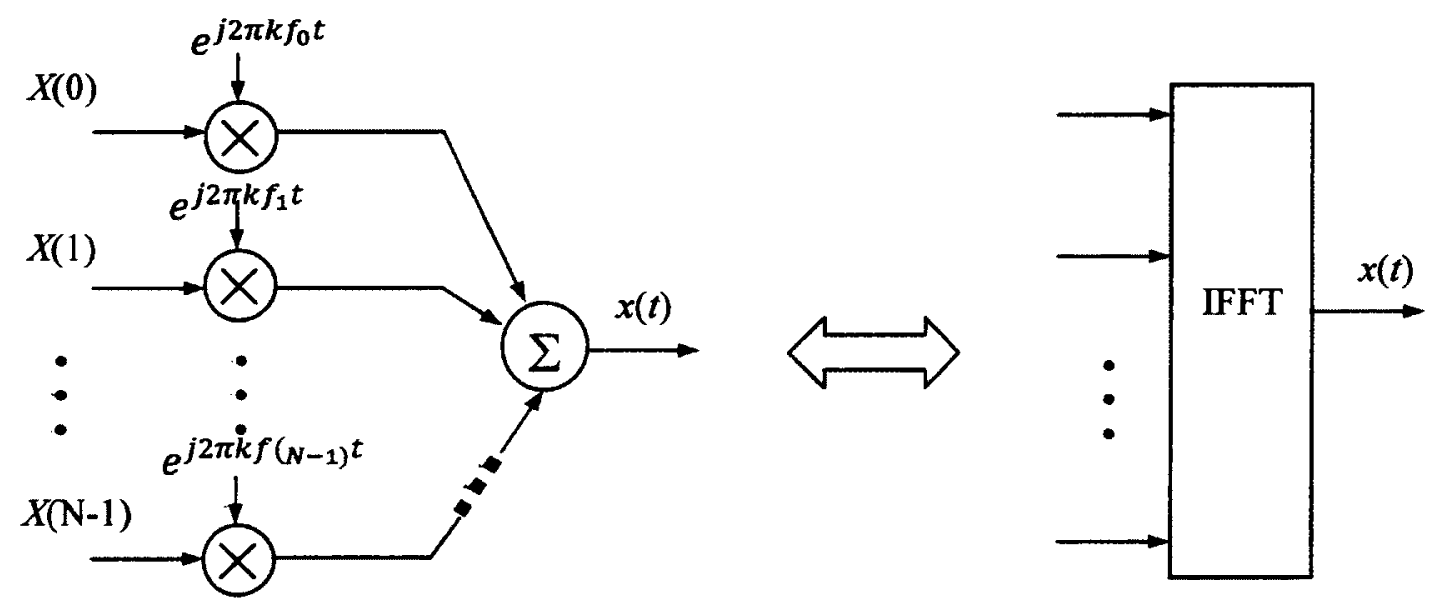

(a) OFDM Transmitter (Modulator)

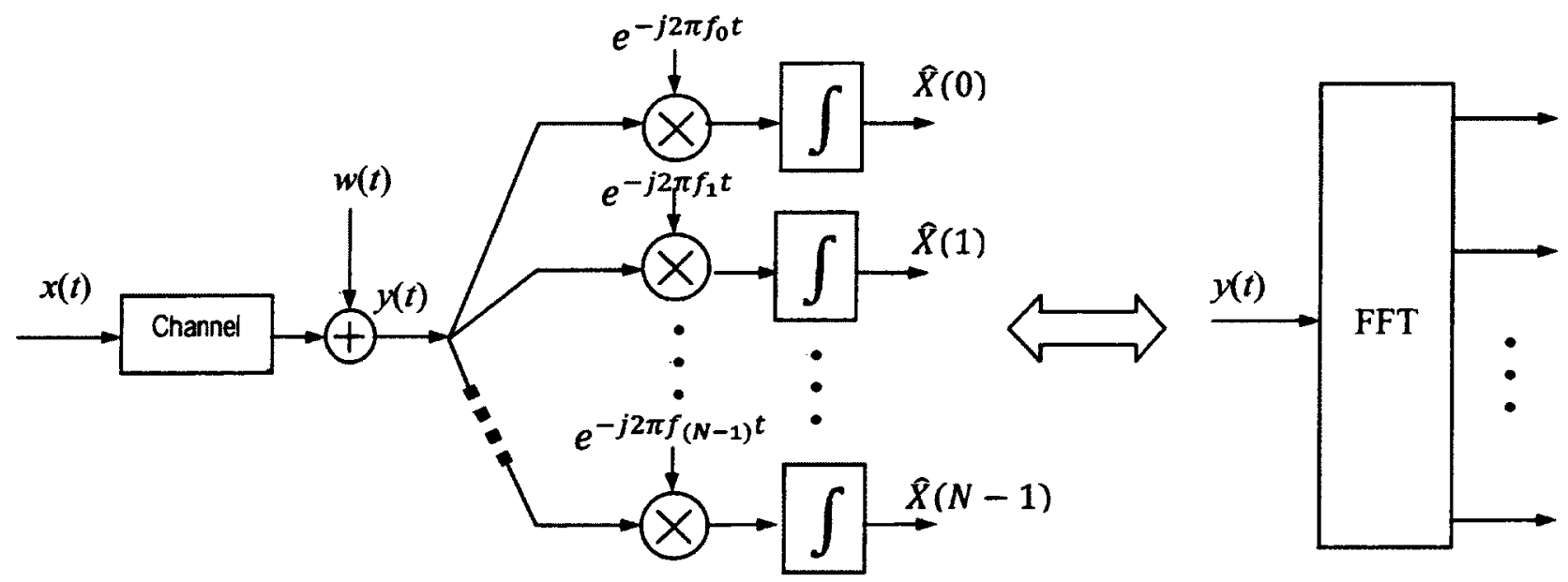

(b) OFDM Receiver (Demodulator)

Figure 2.7. OFDM Transmitter and Receiver 
In LTE, the digital modulation schemes supported are BPSK, QPSK, 16-QAM and 64-QAM and the probability of bit error rate $P_{b}$ and symbol error rate $P_{S}$ are given as follows:

$$
\begin{gathered}
\text { BPSK: } \quad P_{S}=Q\left(2 \sqrt{\frac{E_{S}}{N_{0}}}\right) ; \quad P_{b}=Q\left(2 \sqrt{\frac{E_{S}}{N_{0}}}\right) \\
Q P S K: \quad P_{S} \approx 2 Q\left(\sqrt{\frac{E_{S}}{N_{0}}}\right) ; \quad P_{b} \approx Q\left(2 \sqrt{\frac{E_{S}}{N_{0}}}\right) \\
M-\operatorname{ary} P S K: \quad P_{b}=\frac{1}{\log _{2} M} \operatorname{erfc}\left(\sqrt{\log _{2} M \frac{E_{b}}{N_{0}} \sin ^{2} \frac{\pi}{M}}\right)
\end{gathered}
$$

Rectangular QAM constellations as shown in Figure 2.8 are, in general, sub-optimal since they do not maximally space the constellation points for a given energy. Although, in general, there are a nonrectangular constellation that are optimal for a particular $M$ and which achieve marginally better biterror rate (BER), they are not often used because the rectangular QAMs are much easier to modulate and demodulate. Expressions for the symbol/bit error rate of rectangular QAM are not hard to derive but yield rather unpleasant expressions (Equation 2.4). It is noted that the bandwidth and bandwidth efficiency are given as Bandwidth $\approx 1 / T=1 /\left(\log _{2}(M) T_{b}\right) \mathrm{Hz}$ and Bandwidth efficiency $=\log _{2}(M)$ bps/Hz.

$$
M-\operatorname{ary} Q A M\left(\text { rect const): } P_{b}=\frac{2}{\log _{2} M}\left(1-\frac{1}{\sqrt{M}}\right) \operatorname{erfc}\left(\sqrt{\frac{3 \log _{2} M E_{b}}{2(M-1) N_{0}}}\right)\right.
$$

On the other hand, it is rather hard to establish expressions for the symbol/bit error rates of nonrectangular QAM since it necessarily depends on the constellation, which can be constructed in many different ways.

Higher-order modulations ( $M$ large) are more spectrally efficient but less power efficient (i.e. BER higher). M-QAM are more spectrally efficient than M-PSK but also more sensitive to system nonlinearities. Mobile radio systems are bandwidth limited, therefore PSK is more suited. 


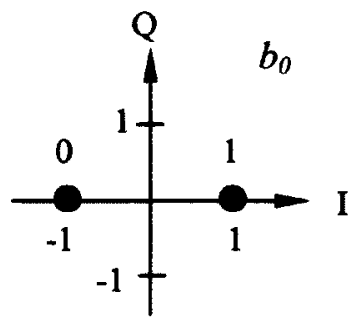

(a) Gray Mapping for BPSK
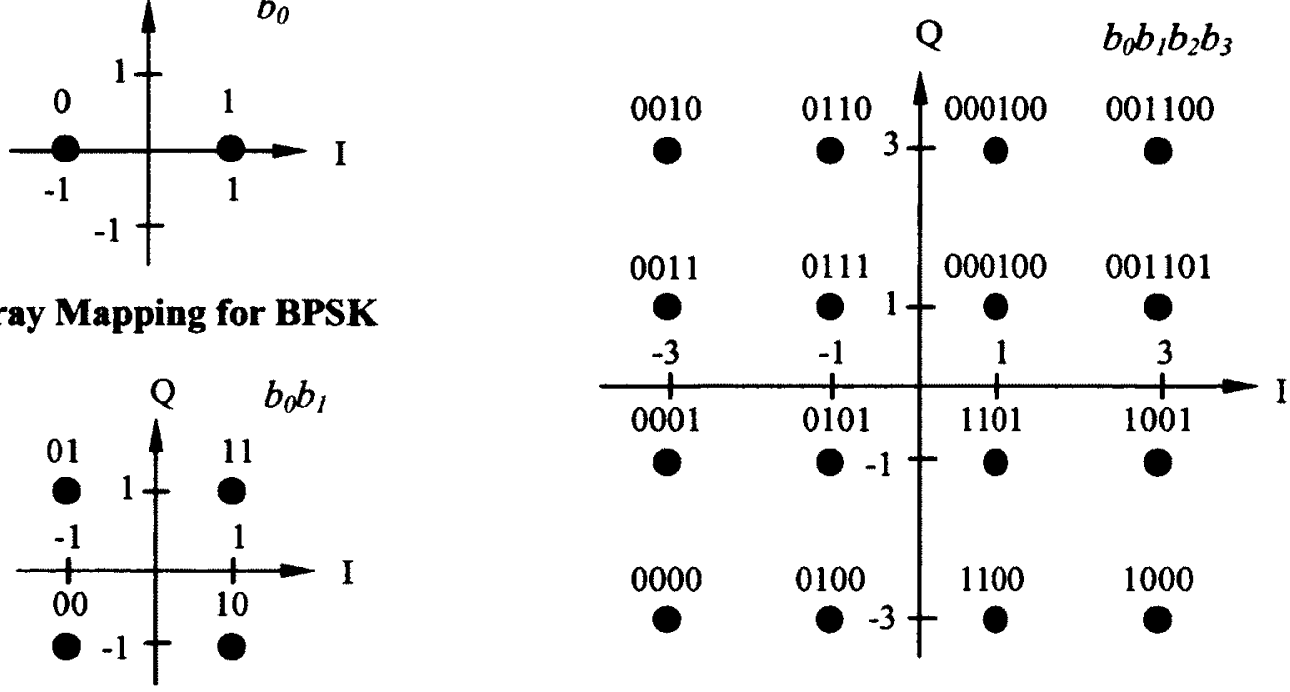

(b) Gray Mapping for QPSK

(c) Gray Mapping for 16-QAM

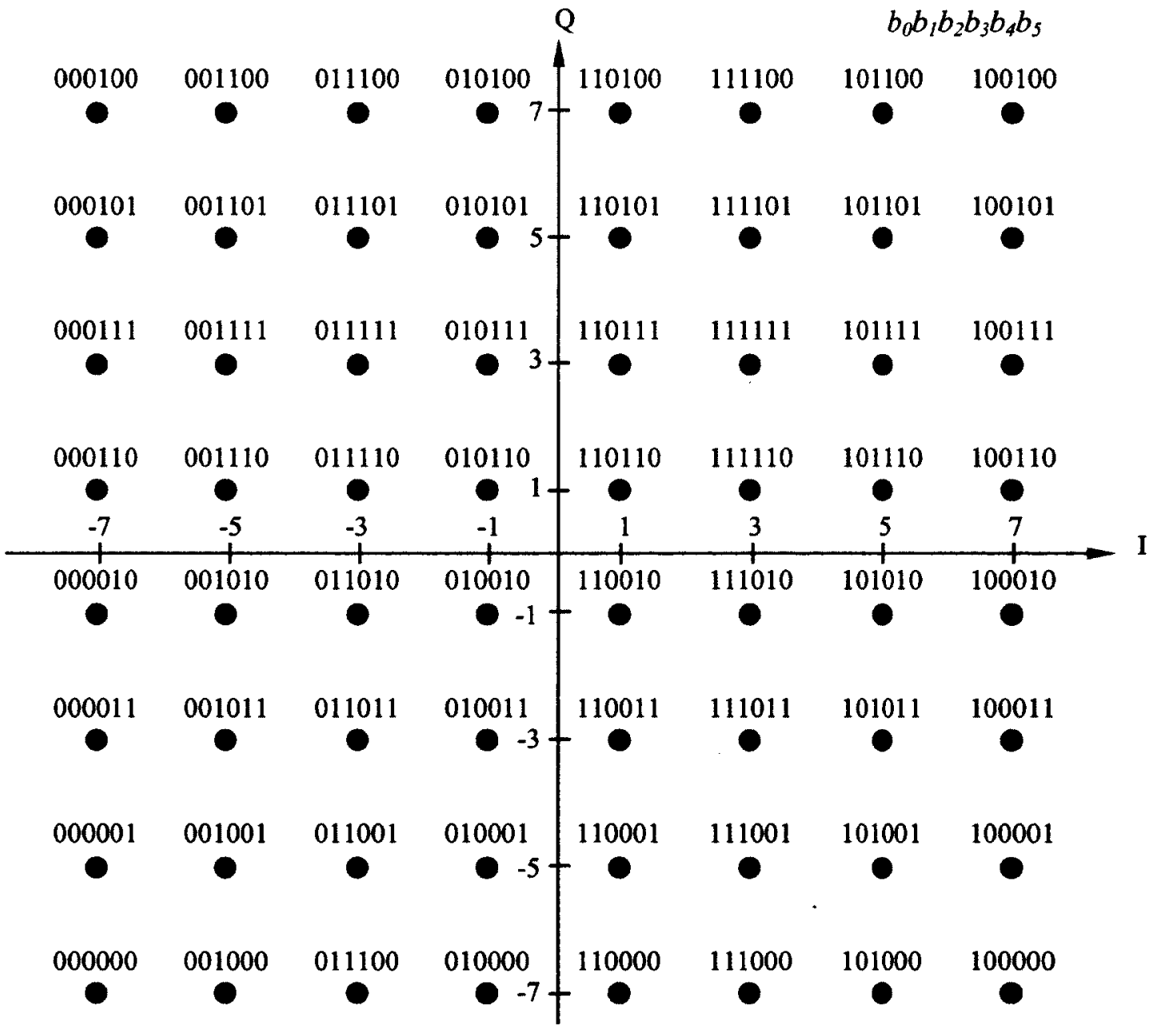

(d) Gray Mapping for 64-QAM

Figure 2.8. Modulation Schemes for LTE/LTE-Advanced 
The duplexing techniques and modes supported in LTE are: TDD, FDD, and half-duplex FDD. TDLTE is typically used in situations where the uplink and downlink data transmissions are not symmetrical (which is usually the case when using wireless phones). The motivation behind TD-LTE are: Internet applications are asymmetric in bandwidth usage where FDD is generally symmetric in bandwidth assignment, TDD allows flexible (system) allocation of bandwidth between uplink and downlink, TDD allows more effective MIMO techniques, TDD channels are reciprocal and hence allow a larger and more efficient set of MIMO techniques, TDD transceiver design is cheaper and less complex.

The advantages of TD-LTE are: TD spectrum is less expensive than FD, since the transmitting and receiving is done using one frequency, the channel estimations for beamforming (and other smart antenna techniques) apply for both the uplink and the downlink, and the capability of dynamic change in the UL and DL capacity ratio to match demand. It offers operators flexibility in adjusting the DL/UL ratio. This feature allows operators to configure the DL/UL ratio to suit the traffic ratio on their network.

The disadvantages of TD-LTE includes a TDD mode of requiring a thorough synchronization of transmissions in the time-domain. A second disadvantage of TDD is the need to use guard periods between the downlink and uplink transmissions. TD-LTE has poor coverage (up to $40 \%$ less) compared to FDD and requires base station synchronization to avoid cross slot interference. Finally, the use of a single channel for full duplex data transmission that prevents download when uploading and vice versa.

FD-LTE is typically used in situations where the uplink and downlink data transmissions are symmetrical (which is not usually the case for wireless phones). 
Table 2.1 shows the FDD and TDD duplexing techniques comparison in LTE.

The advantages of FD-LTE include: reduced interference between neighbouring Radio Base Stations (RBSs) than with TDD, spectral efficiency (which is a function of how well a given spectrum is used by certain access technology) is greater than TDD, and the use for two separate channels to carry data, one for upload and the other for download.

The disadvantages of FD-LTE include: it requires a paired spectrum with sufficient frequency separation to allow simultaneous transmission and reception, a diplexer is needed increasing the cost, and the UL / DL capacity is determined by the frequency allocation set out by the regulatory authorities. It is therefore not possible to make dynamic changes to match capacity.

\begin{tabular}{|c|c|c|}
\hline Duplexing & LTE-FDD & LTE-TDD \\
\hline Spectrum & $\begin{array}{c}\text { 2 separate channels (guard band) } \\
\text { Larger spectrum required }\end{array}$ & $\begin{array}{c}\text { Single channel (guard time) } \\
\text { Smaller spectrum required }\end{array}$ \\
\hline Duplex & Full-duplex & Half-duplex \\
\hline Frequency Reuse Factor & 1 (possible) & Frequency planning required \\
\hline Transmission Efficiency & High & Low \\
\hline Flexibility & Low (by fixed frequency band) & $\begin{array}{c}\text { High (dynamic allocation, } \\
\text { instantaneous peak rate) }\end{array}$ \\
\hline Complexity/Cost & High (Rx filter, etc.) & Low \\
\hline Coverage & $\begin{array}{c}\text { Macro/micro (wide area) } \\
\text { "non degraded", support high } \\
\text { speed mobility }\end{array}$ & $\begin{array}{c}\text { Micro/pico, "degradation of } \\
\text { bandwidth over distance" (cell } \\
\text { area 30\% reduction due to } \\
\text { propagation delay \& low power), } \\
\text { difficult to support high speed }\end{array}$ \\
\hline Uplink Synchronization & Difficult & $\begin{array}{c}\text { Easy } \\
\text { Technique required to distinguish } \\
\text { UL/DL signals }\end{array}$ \\
\hline Miscellaneous & Low latency & $\begin{array}{c}\text { Symmetric channel } \\
\text { (SA, LA can be easily applied) }\end{array}$ \\
\hline
\end{tabular}

Table 2.1. LTE-FDD vs. LTE-TDD

Generally speaking, LTE-FDD is intended for macro cell which supports high speed mobility \& wide coverage; whereas LTE-TDD is intended for picocell (hot spot) which supports high speed transmission \& low power. 


\subsubsection{Multiple Input Multiple Output (MIMO)}

Multiple-Input and Multiple-Output (MIMO), is the use of multiple antennas at both the transmitter and receiver to improve communication performance. It is one of several forms of smart antenna technology. Note that the terms input and output refer to the radio channel carrying the signal, not to the devices having antennas. MIMO techniques are divided in three main categories: spatial diversity (realized by space-time coding), spatial multiplexing, and smart antennas (beamforming) techniques as shown in Figure 2.9.

\section{Spatial Diversity}

Multiple antennas separated in the spatial-domain are used to offer space diversity provided that richscattering environments create uncorrelated multipath fading in different spatial streams.

In fact, space-time coding realizes spatial diversity by transmit- ting signals through multiple transmit antennas. A space-time code (STC) is a method employed to improve the reliability of data transmission in wireless communication systems using multiple transmit antennas. STCs rely on transmitting multiple, redundant copies of a data stream to the receiver in the hope that at least some of them may survive the physical path between transmission and reception in a good enough state to allow reliable decoding.

\section{Spatial Multiplexing (SM)}

Spatial multiplexing (SM) is a transmission technique in MIMO wireless communication to transmit independent and separately encoded data signals, so-called streams, from each of the multiple transmit antennas. Therefore, the space dimension is reused, or multiplexed, more than one time. 


\section{Beamforming}

Beamforming is a signal processing technique used in sensor arrays for directional signal transmission or reception. This is achieved by combining elements in the array in a way where signals at particular angles experience constructive interference while others experience destructive interference. Beamforming can be used at both the transmitting and receiving ends in order to achieve spatial selectivity. The improvement compared with an omni-directional $\mathrm{Rx} / \mathrm{Tx}$ is known as the receive/transmit gain (or loss).

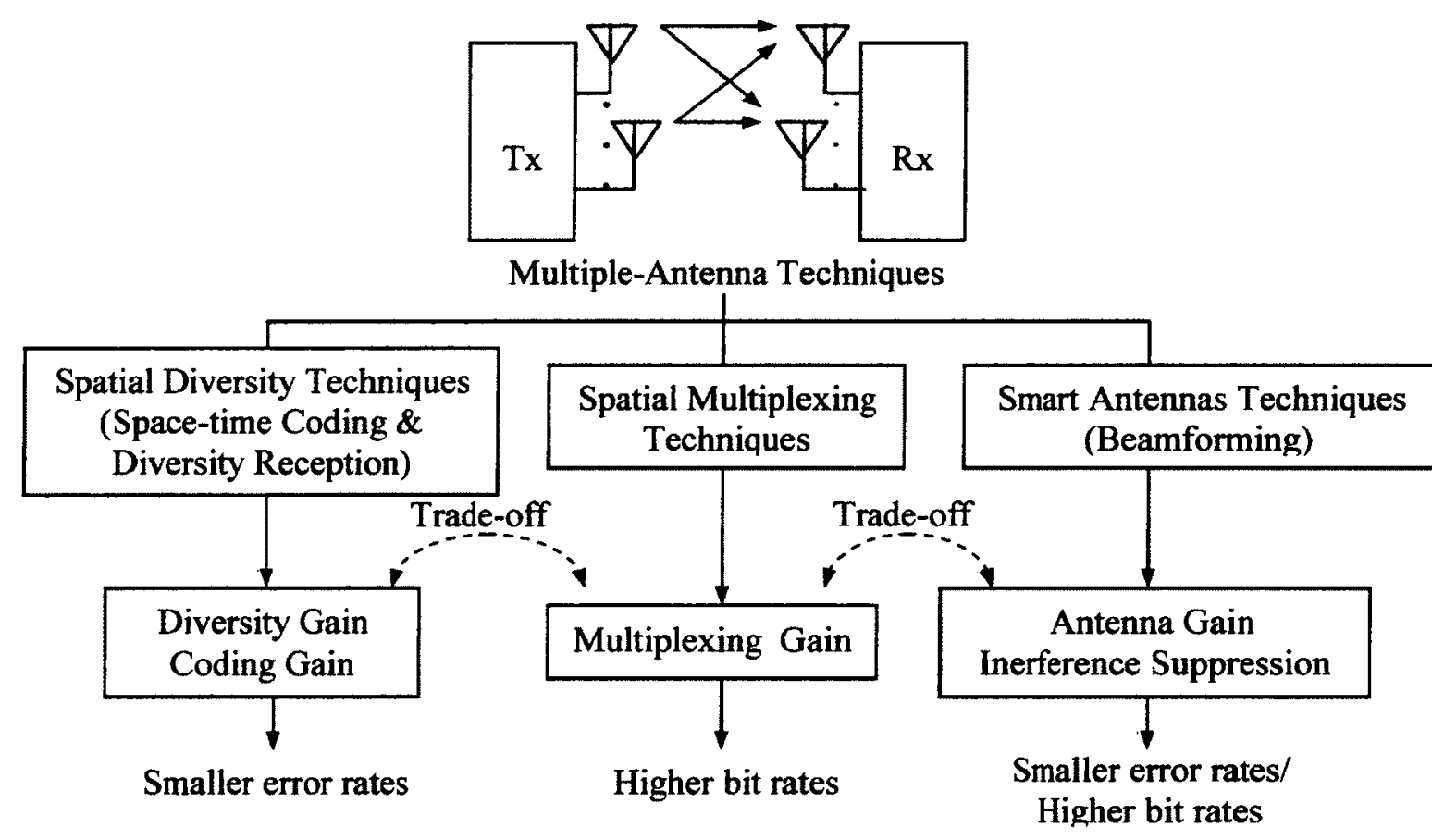

Figure 2.9. MIMO Techniques

In MIMO techniques, there is a trade-off between spectral efficiency (high data rates) and power efficiency (small error rates), given fixed bandwidth \& transmission power. 


\subsection{Interferences and Uncertainties in OFDM}

There are two different sources of interference (crosstalk) can be identified in the OFDM system.

\subsubsection{Inter-Symbol Interference (ISI)}

Inter-Symbol Interference is the crosstalk between signals within the same subchannel of consecutive FFT frames (which are separated in time by the signaling interval $T$ ) due to multipath, as shown in Figure 2.10. It is noted that ISI gets worse as data rate increases. Multipath-induced time delay spread results in ISI.

Signal received by direct path

Delayed signal received via longer path

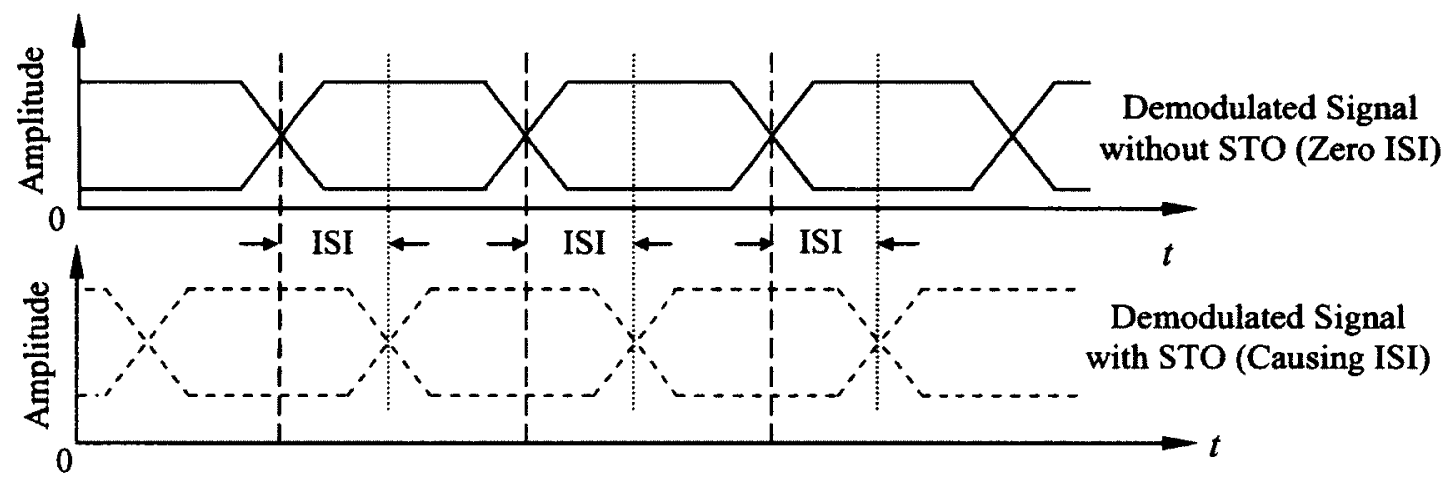

Figure 2.10. Time-Domain - Multipath-Induced Time Delay Results in ISI

\subsubsection{Inter-Carrier Interference (ICI)}

Inter-Carrier Interference is the crosstalk between adjacent subchannels or frequency bands of the same FFT frame caused by Doppler shift in OFDM modulation (loss of orthogonality) as illustrated in Figure 2.11. Tx and Rx LO-induced frequency/phase mismatch or motion-induced Doppler shift results in ICI.
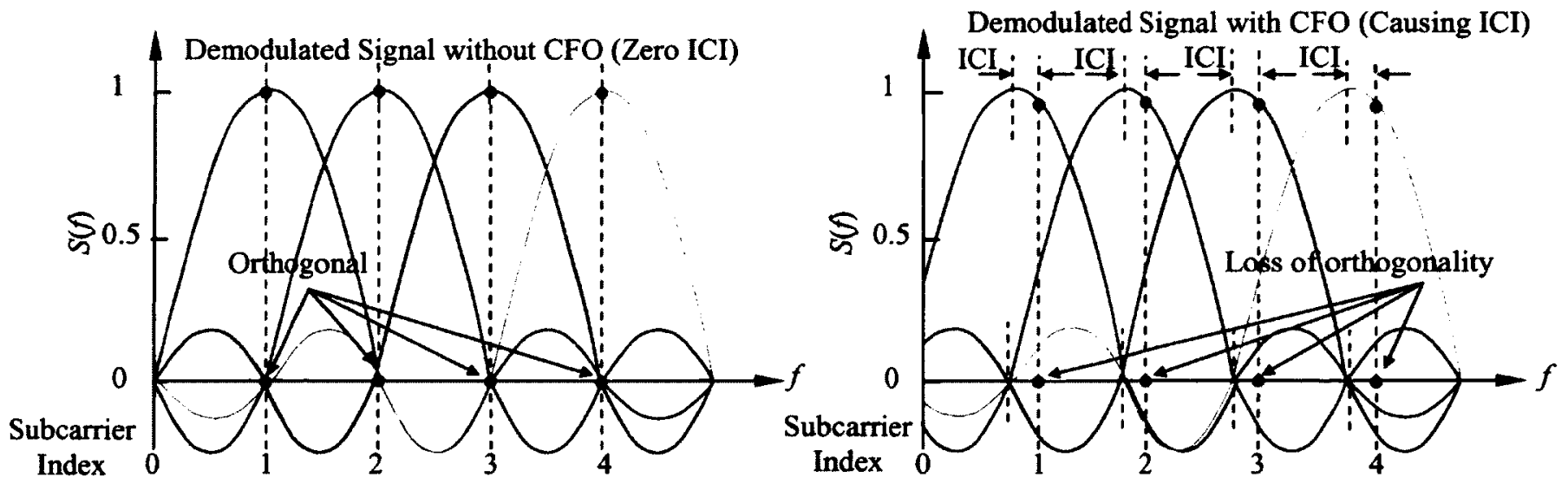

Figure 2.11. Frequency-Domain - Tx/Rx LO Mismatch/Motion-induced Doppler Shift CFO ICI 
There are two uncertainties (ambiguities) in the receiver of OFDM symbol: the uncertainty in the arrival time of the OFDM symbol (such an ambiguity gives rise to a rotation of the data symbols); and the uncertainty in carrier frequency (a difference in the Tx/Rx LOs gives rise to a shift of all the subcarriers).

\subsubsection{Symbol Time Offset (STO) Effect}

The timing error offset is due to the propagation delay. Symbol Time Offset is the uncertainty of the arrival time of the OFDM symbol which gives rise to a rotation of the data symbols in the constellation circle. This uncertainty is modeled as a delay in the channel impulse response $\delta(k-\tau)$, where $\tau$ is the integer-valued unknown arrival time of a symbol as shown in Table 2.2.

\subsubsection{Carrier Frequency Offset (CFO) Effect}

The frequency error offset arises from the mismatch between transmitter and receiver oscillators and Doppler effects. CFO is the uncertainty of the carrier frequency in the OFDM symbols which gives rise to a shift of all subcarriers. Notice that all subcarriers experience the same shift $\varepsilon$. This uncertainty is modeled as a complex multiplicative distortion of the received data in the time domain $e^{j 2 \pi \varepsilon / N}$, where $\varepsilon$ denotes the difference in the transmitter and receiver oscillator frequencies as a fraction of the intercarrier spacing (i.e., normalized CFO $\varepsilon=f_{\text {offset }} / \Delta f$ ). Let $\tau$ and $\varepsilon$ denote the normalized STO and CFO respectively. The received baseband signal under the presence of STO $\tau$ and CFO $\varepsilon$ can be expressed as:

$$
y[n]=\frac{1}{N} \sum_{k=0}^{N-1} H[k] X[k] e^{j \frac{j 2 \pi}{N}(k+\varepsilon)(n+\tau)}+w[n]
$$

A STO $\tau$ incurs the phase offset of $2 \pi k \tau / N$ in the frequency-domain, which is proportional to the subcarrier index $k$ and the STO $\tau$. Note that it is equivalent to a time shift of $+\tau$ in the time-domain signal $x[n]$. The effect of STO with no CFO $(\varepsilon=0)$ is:

$$
y[n]=\sum_{k=0}^{N-1} H[k] X[k] e^{j \frac{j 2 \pi k}{N}(n+\tau)}
$$


Table 2.2 shows that a CFO $\varepsilon$ causes a phase offset of $2 \pi n \varepsilon / N$ in the time-domain which is proportional to the time index $n$ and the CFO $\varepsilon$. Note that it is equivalent to a frequency shift of $-\varepsilon$ in the frequencydomain signal $X[k]$. The effect of CFO with no STO $(\tau=0)$ is:

$$
y[n]=\frac{1}{N} \sum_{k=0}^{N-1} H[k] X[k] e^{j \frac{j 2 \pi}{N}(k+\varepsilon) n}+w[n]
$$

A time-dispersive channel can be characterized in the time-domain by its channel impulse response (CIR):

$$
h[n]=\sum_{i=0}^{L-1} \alpha_{i} \delta\left[n-\tau_{i} i k\right]
$$

where $\alpha_{i}$ is path gain of path $i ; \tau_{i}$ is path delay of path $i$; $L$ is number of multipath components. In the frequency-domain by its channel transfer function (CTF):

$$
H(k)=\frac{1}{N} \sum_{n=0}^{N-1} h[n] \cdot e^{-j \frac{2 \pi n k}{N}}
$$

The phenomenon of multipath fading causes a previous symbol to interfere with a latter symbol, known as the inter-symbol interference (ISI). The received signal is the convolution of the transmitted symbols and the discrete-time channel impulse response (CIR). Since the cyclic prefix converts linear convolution into circular convolution, we need to perform a circular convolution:

$$
\text { symbol } \otimes \text { channel } \Leftrightarrow \text { FFT(symbol) x FFT(channel) }
$$

\begin{tabular}{|c|c|c|c|}
\hline & Received Signal & $\operatorname{STO}(\tau)$ & CFO (E) \\
\hline Time-Domain Signal & $y(n)$ & $x(n+\tau)$ & $e^{j 2 \pi \varepsilon n / N} \cdot x(n)$ \\
\hline Frequency-Domain Signal & $Y(k)$ & $e^{j 2 \pi \tau k N} \cdot X(k)$ & $X(k-\varepsilon)$ \\
\hline
\end{tabular}

As a result the division by the FFT (channel) can undo the channel distortion.

Table 2.2. Effects of CFO and STO 


\subsection{Mobile Radio Channel Characterization}

In wireless systems, fading may either be due to (1) multipath propagation and time variant fading (Doppler spread), referred to as small-scale fading, or due to (2) shadowing from obstacles affecting the wave propagation and path loss, referred to as large-scale fading. This is shown below in Figure 2.12.

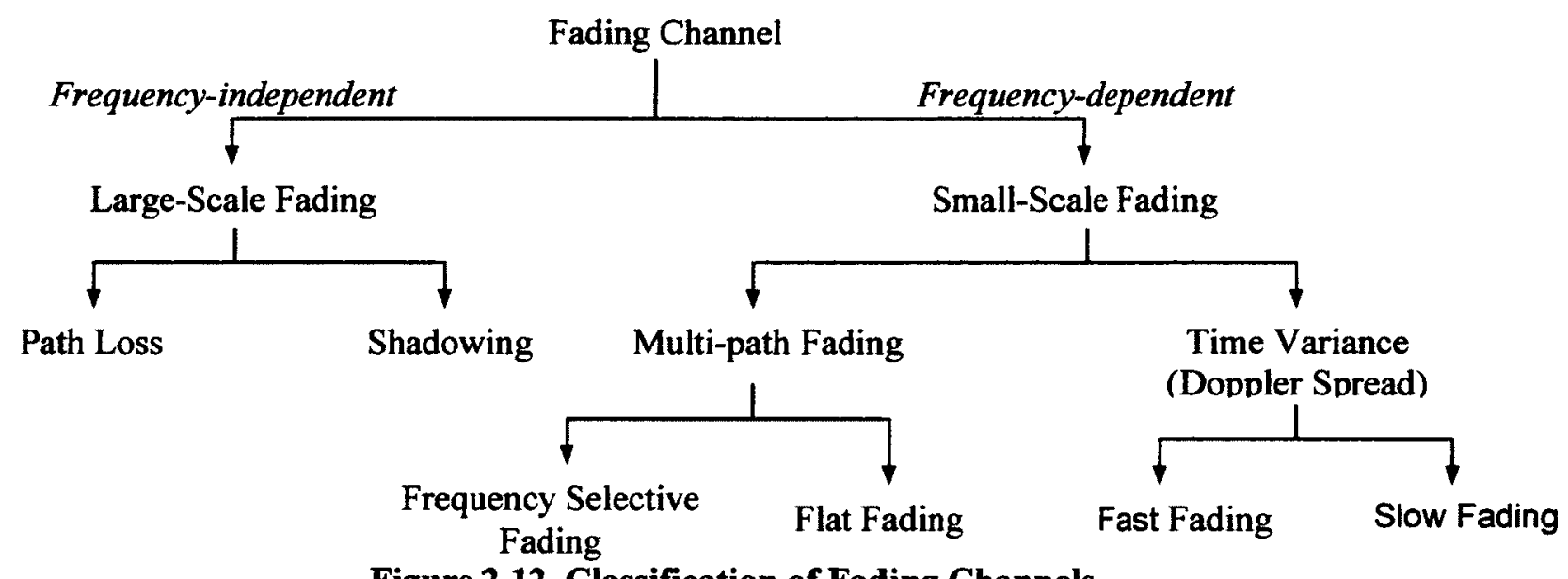

Figure 2.12. Classification of Fading Channels

\subsubsection{Small-Scale Fading (Frequency-Dependent)}

Small-scale fading refers to the rapid changes in radio signal amplitude in a short period of time or travel distance. Small-scale fading is due to the constructive and destructive interference of the multiple signal paths between the transmitter and receiver. This occurs at a spatial scale of the order of the carrier wavelength, and is frequency dependent. Small-scale multipath fading is most important in the design of reliable and efficient communication systems. It is caused by the superposition or cancellation of multipath propagation signals, on the speed of the transmitter or receiver, and on the bandwidth of the transmitted signal. It is also known as Multipath Fading or Rayleigh/Ricean Fading. Rayleigh fading is most applicable when there is no dominant propagation along a line of sight between the transmitter and receiver. If there is a dominant line of sight, Rician fading may be more applicable. It is noted that the Rician K-factor specifies the ratio of specular-to-diffuse power for a direct line-of-sight path. The ratio is expressed linearly, not in $\mathrm{dB}$. For Rician fading, the $\mathrm{K}$-factor is typically between 1 and 10 . A K-factor of 0 corresponds to Rayleigh fading. Figure 2.13 shows Rayleigh and Rician fadings for $f_{\mathrm{D}}=100 \mathrm{~Hz}$. 


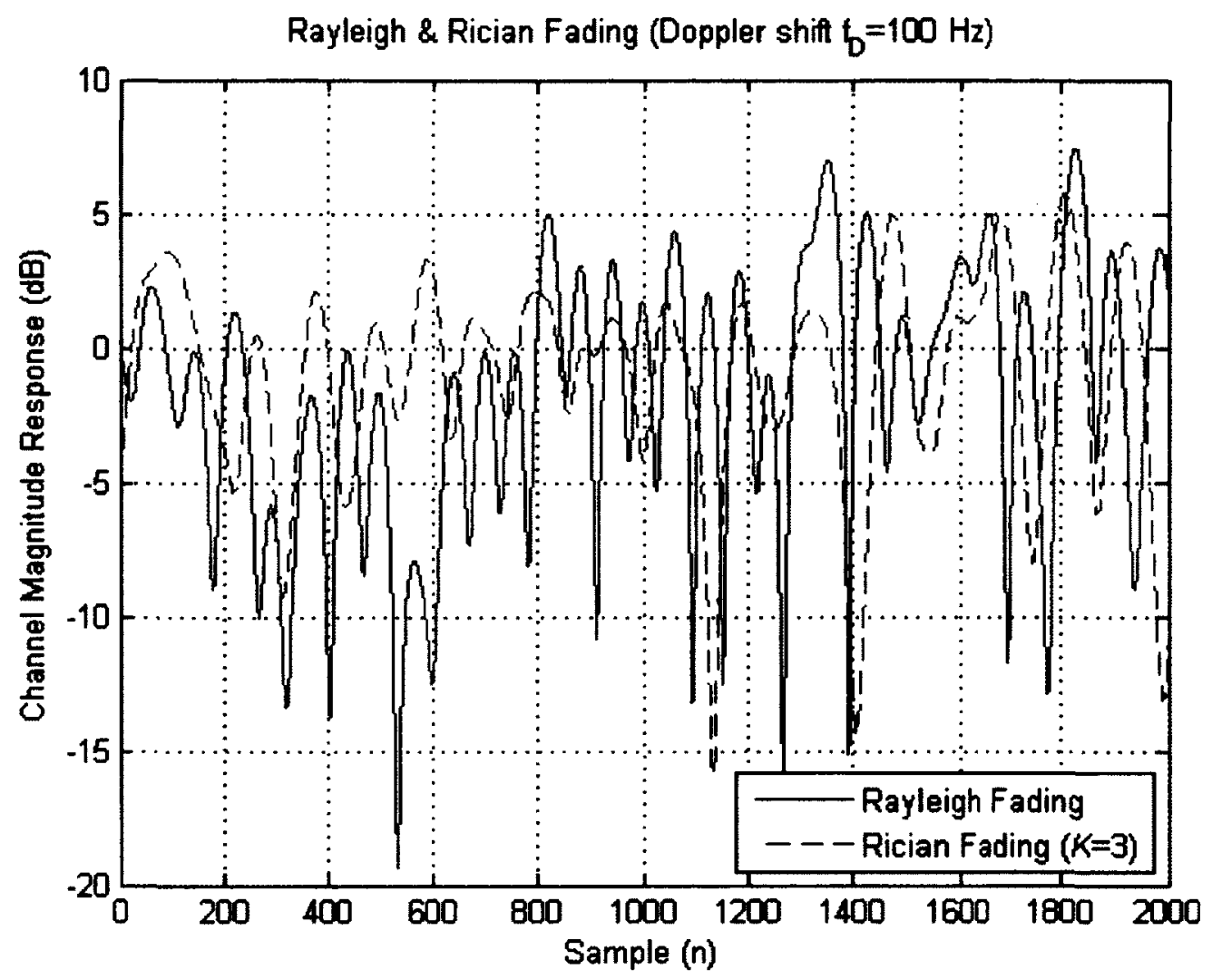

Figure 2.13. Rayleigh \& Rician Fading

In flat fading, the coherence bandwidth $B_{C}$ of the channel is larger than the bandwidth of the signal $B_{S}$ (or alternatively the channel delay spread $\tau_{\mathrm{RMS}}$ is smaller than the signal symbol time $T_{\mathrm{Sym}}$ ). Hence there is no ISI since there is only a low delay spread. Therefore, all frequency components of the signal will experience the same magnitude of fading as shown in Figure 2.14.

In frequency-selective fading (Figure 2.15), the coherence bandwidth $B_{C}$ of the channel is smaller than the bandwidth of the signal $B_{S}$ (or alternatively the channel delay spread $\tau_{\mathrm{RMS}}$ is greater than the signal symbol time $T_{\mathrm{Sym}}$ ). Hence there is ISI since high delay spread. Different frequency components of the signal therefore experience decorrelated fading. Frequency-selective fading is caused by multipath propagation of the channel causing uneven gains at different frequencies. 


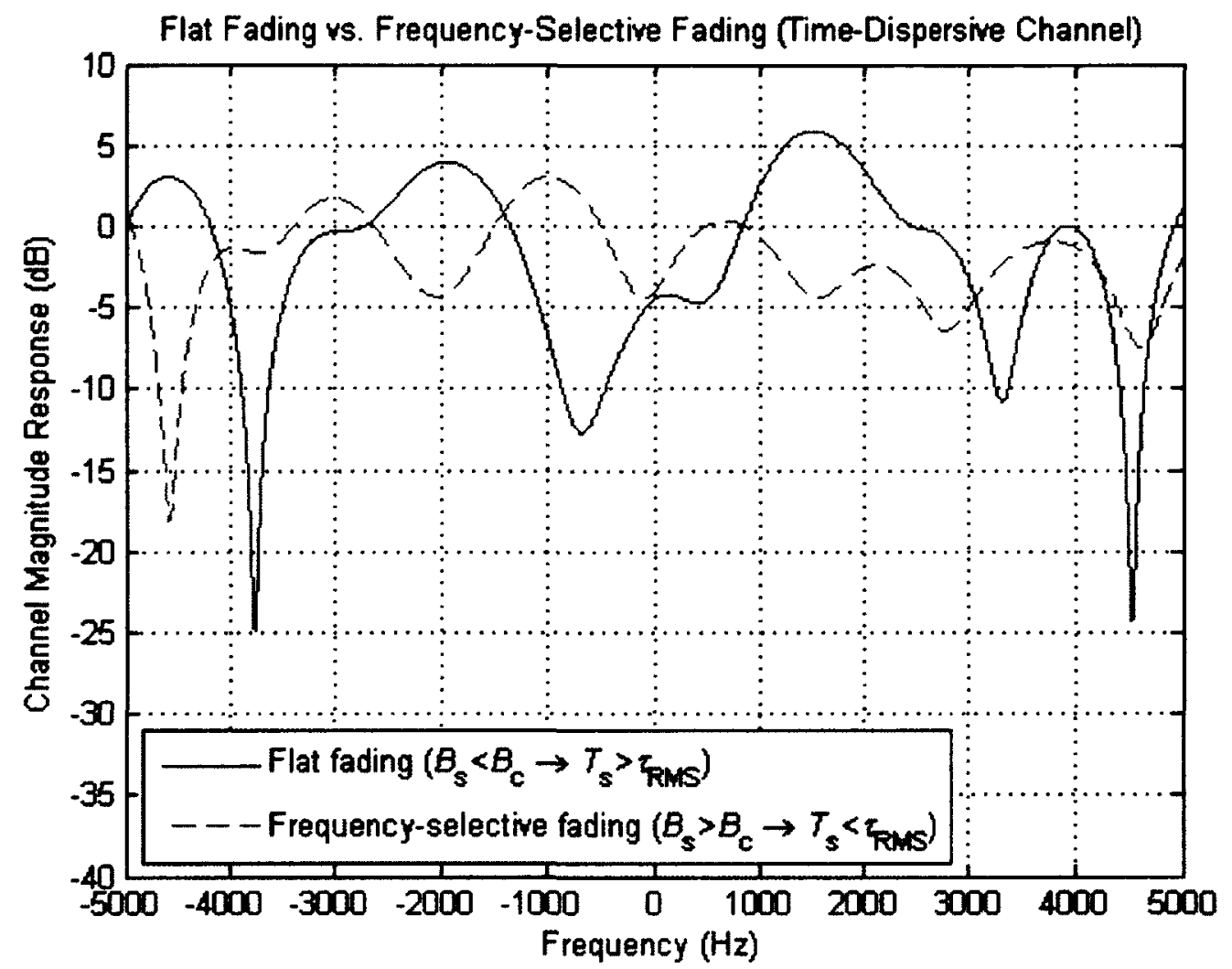

Figure 2.14. Flat Fading vs. Frequency-Selective Fading (Multipath Delay Spread)

Multipath delay spread leads to time-dispersion and frequency-selective fading, whereas motion or Doppler spread leads to frequency-dispersion and time-selective fading. These two propagation mechanisms are independent. For broadband cellular and vehicular environment, we need fast fading and frequency-selective fading channel models. Typically, delay spread values for indoor at $50 \mathrm{~ns}$ for indoor scenarios and $1 \mu \mathrm{s}$ for outdoor/cellular applications. Also typical coherence bandwidths for indoors are $20 \mathrm{MHz}$ and for outdoor/cellular are $500 \mathrm{kHz}$. The implication is that at high data rates the symbol smears onto the adjacent ones causing ISI.

Flat fading means no multipath ISI effects, and is found in narrowband and indoors applications. Frequency-selective fading implies multipath ISI effects and is found in broadband and outdoor applications. Slow fading implies no Doppler effects and is found in indoor Wi-fi home networking. Fast fading means Doppler effects, time-selective channel and is found in cellular, vehicular applications. 
The figures 2.15, 2.16, and 2.17 [30] show how the channel attenuation depends on time and frequency of the signal for various values of the delay spread and Doppler spread. It can be observed that the rate of variations of the channel attenuation increases if the Doppler spread increases. This coincides with a decrease of the coherence time of the channel. Similarly, the effect of changing the signal frequency increases if the channel has a smaller coherence bandwidth, corresponding to a longer delay spread. It is noted that the time axis is scaled in multiples of the symbol duration $T_{s}$ and the frequency axis in multiples of $2 / T_{s}$. The dispersion of the channel is given by the corresponding normalized parameters $t_{m}=T_{m} / T_{s}$ and $f_{D}=B_{D} T_{s} / 2$. Figure 2.15 shows a highly frequency-selective, but not very time selective amplitude of the channel attenuation in $\mathrm{dB}$ for $t_{m}=0.05$ and $f_{D}=0.005$.

Figure 2.16 shows almost a non-selective frequency but very time-selective: channel having a rapidly fading amplitude. However, in most practical mobile radio systems, the received signal amplitude depends on both frequency and location or time (Figure 2.17).

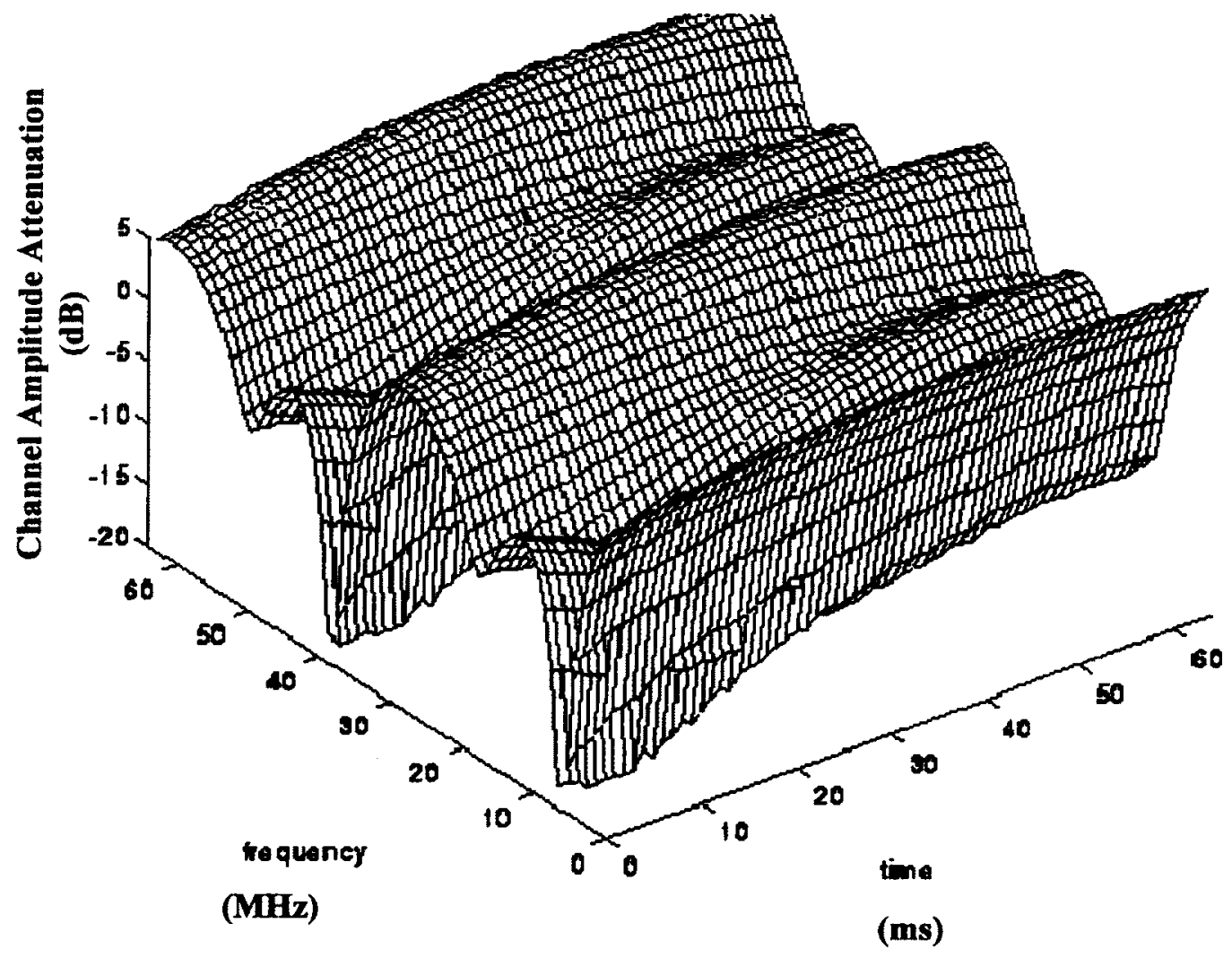

Figure 2.15. Frequency-Selective Fading $\tau_{m}=0.05$ and $f_{D}=0.005$ 

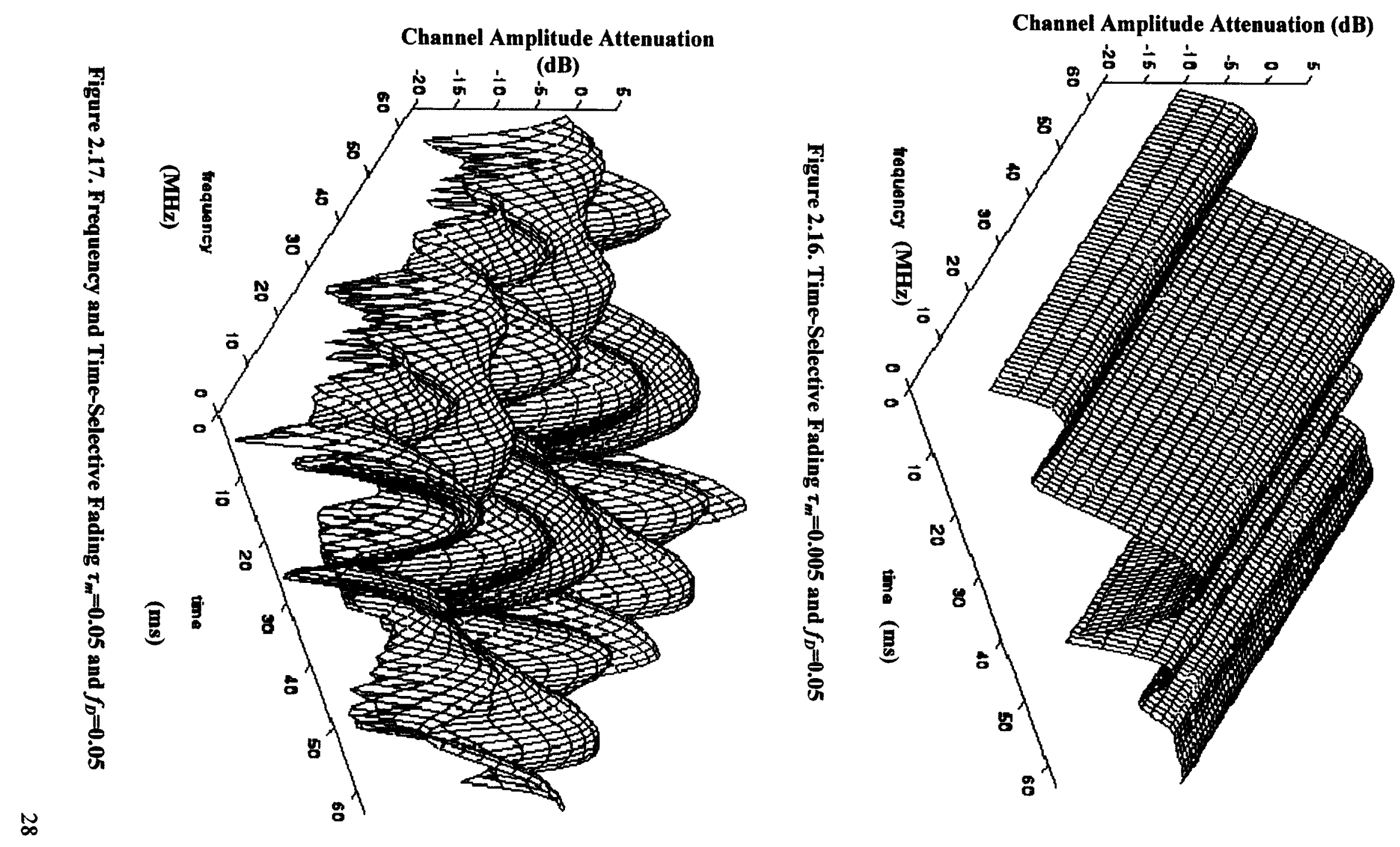
Fast fading or time-selective fading (Figure 2.18) occurs when the channel impulse response $T_{C}$ changes rapidly relative to the baseband symbol duration $T_{\text {sym }}$ (alternatively $B_{\mathrm{S}}<B_{\mathrm{D}}$ ). In this regime, the amplitude and phase change imposed by the channel varies considerably over the period of use and high Doppler spread. Time-selective fading is caused by the Doppler effect in the mobile communication channel, where the terminals are in motion at a certain speed relative to the base station. Time-selective fading is the result of uneven gains occuring at different times. Fast fading is also known as short-term fading, multi-path effect, Raleigh distribution, and microscopic fading. Slow fading (Figure 2.18) arises when the channel impulse response $T_{C}$ changes at a rate much slower than the baseband symbol duration $T_{\text {sym }}$ (alternatively $B_{\mathrm{S}}>B_{\mathrm{D}}$ ). In this regime, the amplitude and phase change imposed by the channel can be considered roughly constant over the period of use and any Doppler spread is low. Slow fading can be caused by events such as shadowing, where a large obstruction such as a hill or large building obscures the main signal path between the transmitter and the receiver. Slow fading is also known as long-term fading, shadowing effect, log-normal distribution, and macroscopic fading.

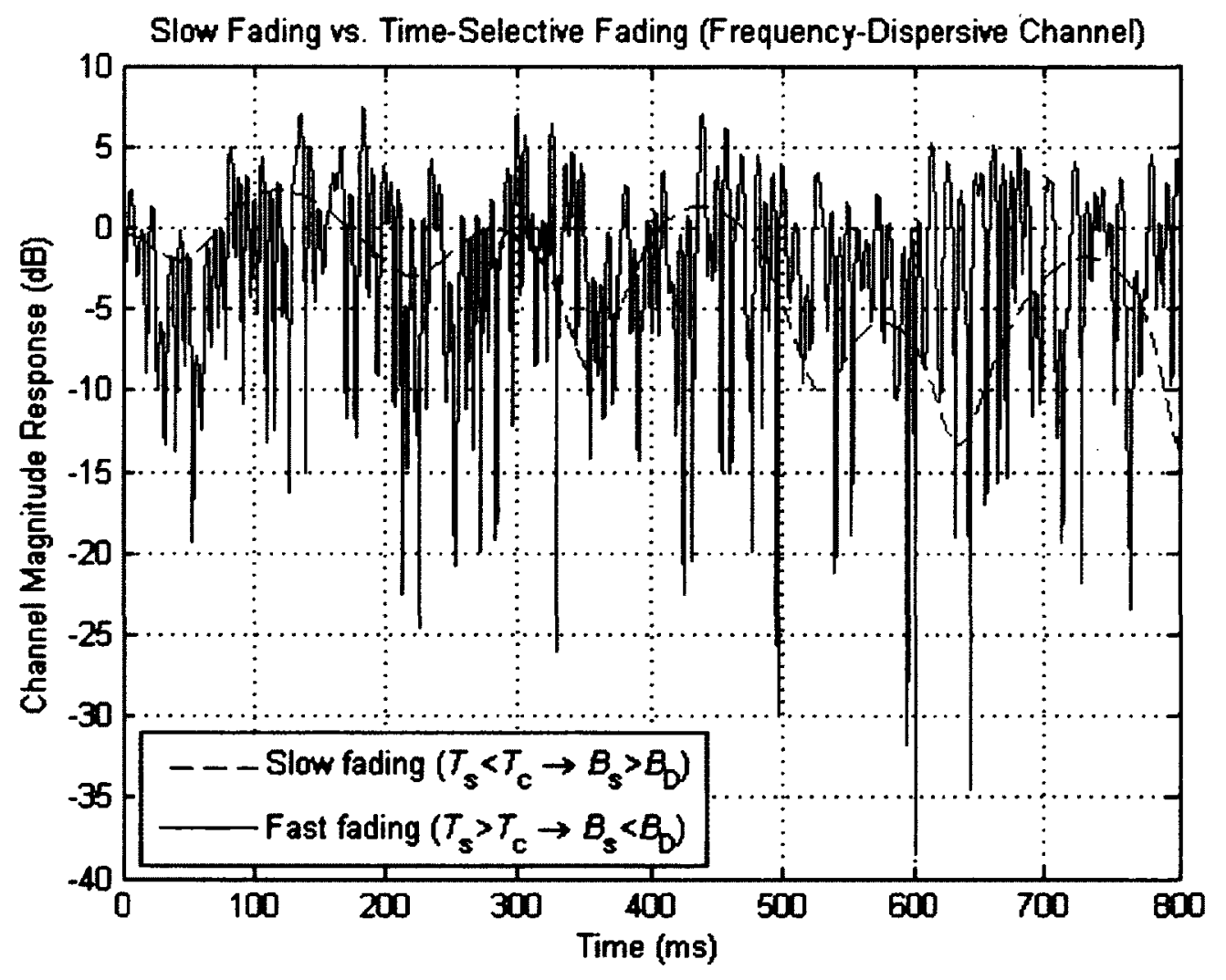

Figure 2.18. Slow Fading vs. Fast Fading (Doppler Spread) 


\subsubsection{Large-Scale Fading (Frequency-Independent)}

Large-scale fading is due to path loss of the signal as a function of distance and shadowing by large objects such as buildings and hills. This is shown in Figure 2.19. This occurs as a mobile moves through a distance of the order of the cell size, and is frequency independent. Large-scale fading is more relevant to issues such as cell-site planning. This is the loss that propagation models try to account for, and is mostly dependant on the distance from the transmitter to the receiver. It is also known as Large Scale Path Loss, Log-Normal Fading or Shadowing. Path loss is caused by dissipation of the power radiated by the transmitter as well as by the effects of the propagation channel. Path loss models generally assume that the path loss is the same at a given transmit-receive distance (assuming that the path-loss model does not include shadowing effects). Shadowing is caused by obstacles between the transmitter and receiver that attenuate the signal power through absorption, reflection, scattering, and diffraction. Shadowing is defined as an obstruction to line-of-sight paths and causes areas of weak received signal strength. In general, large scale path loss decays gradually with distance from the transmitter. Signals vary with time and location and may combine direct and indirect paths. Slow fading comes from the mobility, changes in shadowing or changes in the path (e.g. passing a tree or building), and does not vary quickly with frequency. Fast fading comes from moving through the constructive and destructive interference patterns caused by multipath, and varies quickly with frequency.

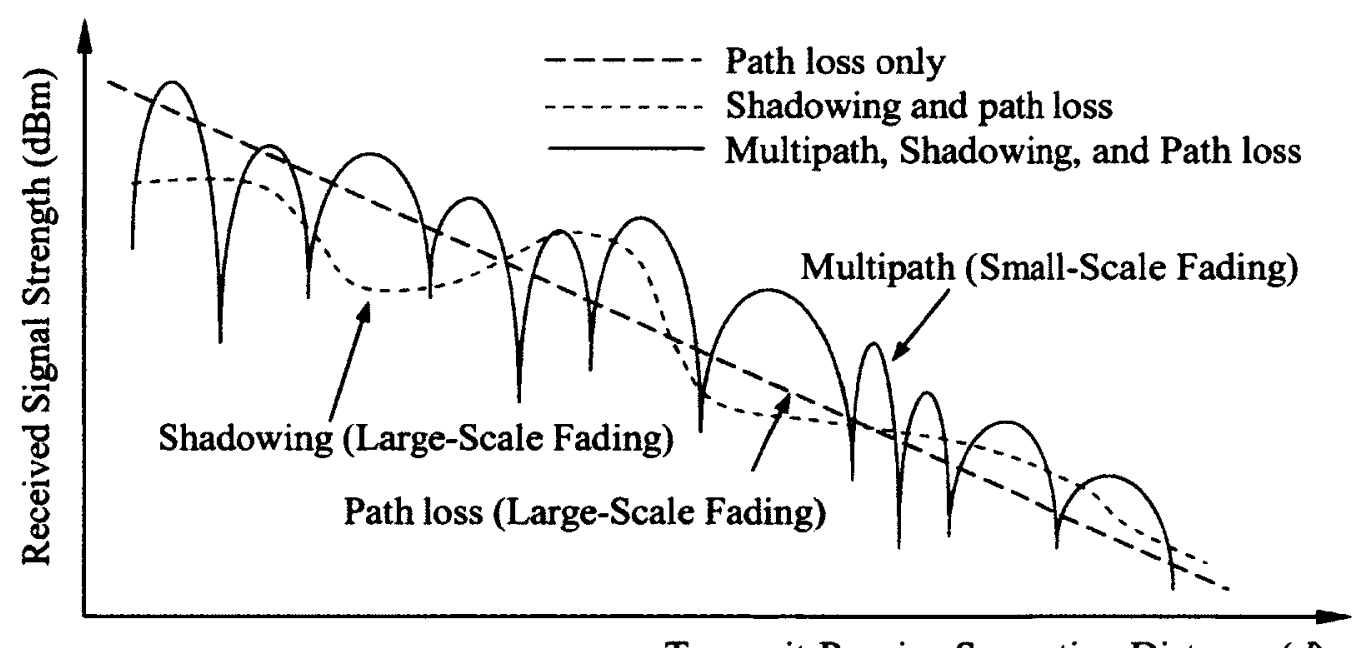

Transmit-Receive Separation Distance (d)

Figure 2.19. Large-Scale Fading vs. Small-Scale Fading 


\subsubsection{Channel Coherence, Spread, Dispersion and Selectivity}

Delay spread ( $\left.\tau_{\mathrm{RMS}}\right)$ is a measure of the multipath richness of a channel as shown in Figure 2.20 [31]. In general, it can be interpreted as the difference between the time of arrival of the first significant multipath component (typically the line-of-sight component) and the time of arrival of the last multipath component. In order to avoid ISI, the symbol time $T_{S y m}$ needs to be much greater than the delay spread $\tau_{R M S}$. Power delay profile conveys the multipath delay spread effects of the channel. RMS delay spread quantifies the severity of the ISI phenomenon. The ratio of RMS delay spread to the data symbol period determines the severity of the ISI. The mean excess delay $(\bar{\tau})$, RMS delay spread $\left(\tau_{\mathrm{RMS}}\right)$, and excess delay spread $\left(\sigma_{\tau}\right)$ are multipath channel parameters (where $a_{k}$ is the path gain and $\tau \mathrm{k}$ is the path delay):

$$
\tau_{R M S}=\sqrt{\frac{\sum_{k=0}^{N-1}\left(\tau_{k}-\bar{\tau}\right)^{2} a_{k}^{2}}{\sum_{k=0}^{N-1} a_{k}^{2}}} ; \quad \bar{\tau}=\frac{\sum_{k} P\left(\tau_{k}\right) \tau_{k}}{\sum_{k} P\left(\tau_{k}\right)}=\frac{\sum_{k=0}^{N-1} \tau_{k} a_{k}^{2}}{\sum_{k=0}^{N-1} a_{k}^{2}} ; \quad \sigma_{\tau}=\sqrt{\overline{\tau^{2}}-(\bar{\tau})^{2}}
$$

These parameters can be determined from a power delay profile $P(\tau)$ :

$$
P(t)=\sum_{k=0}^{N-1} a_{k}^{2} \delta\left(t-\tau_{k}\right)
$$

A rule of thumb for the delay profile is that it is equal to four times the length of the delay spread $\Delta \tau \approx$ $4 \tau_{\text {RMS }}$.

Coherence bandwidth $\left(B_{\mathrm{C}}\right)$ is a statistical measure of the range of frequencies over which the channel can be considered "flat". This is also shown in Fig. 2.20 [31]. The coherence bandwidth, the approximate maximum bandwidth or frequency interval over which two frequencies of a signal are likely to experience comparable or correlated amplitude fading. For a channel with exponential delay spread:

$$
B_{C} \simeq \frac{1}{5 \tau_{R M S}} \simeq \frac{1}{\tau_{\max }}
$$

The coherence bandwidth is a measure of the "good" frequency range, the bandwidth over which the channel appears flat, or non-distorting. Frequency components of a signal that are separated by more than the coherence bandwidth are affected differently and are thus uncorrelated. By contrast, components separated by an amount less than $B c$ observe what is known as a flat channel as given in Equation 2.14. 

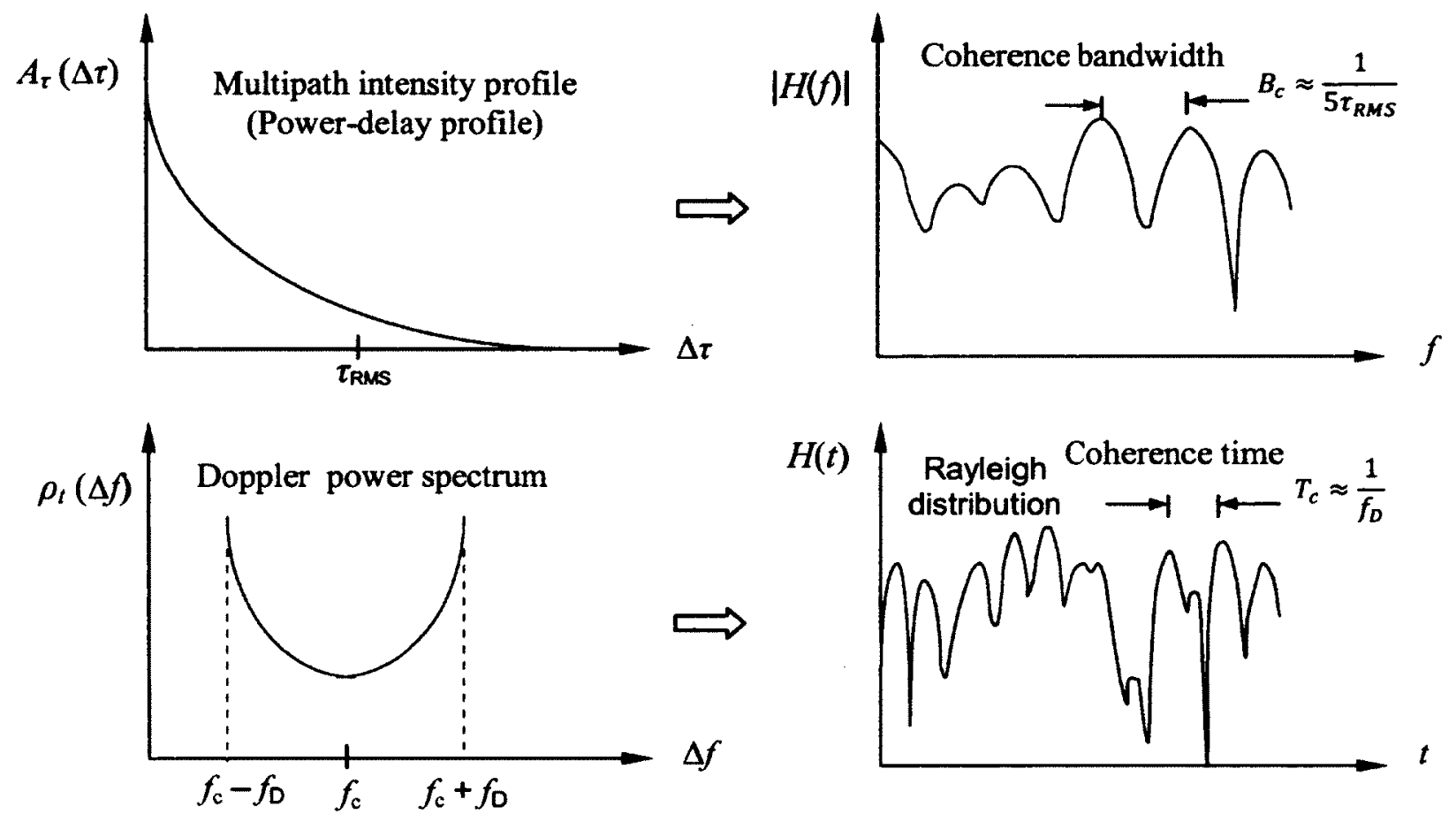

$H(t)$

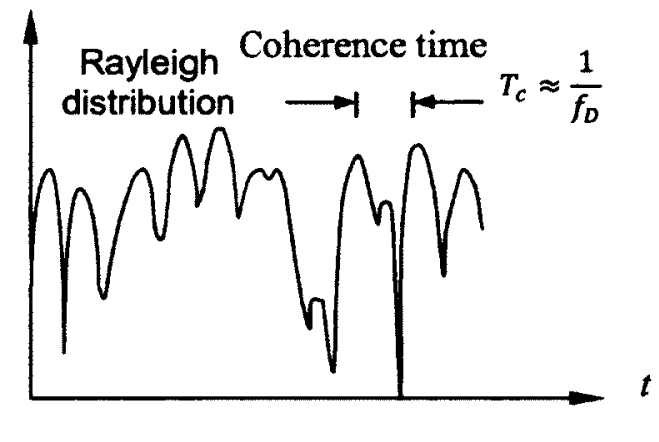

Doppler power spectrum $\rho_{t}(\Delta f)$ determines the Correlation time of the channel.

Multipath intensity profile $A_{\tau}(\Delta \tau)$ determines the Correlation bandwidth of the channel.

Figure 2.20. Power-Delay Profile vs. Doppler Power Spectrum
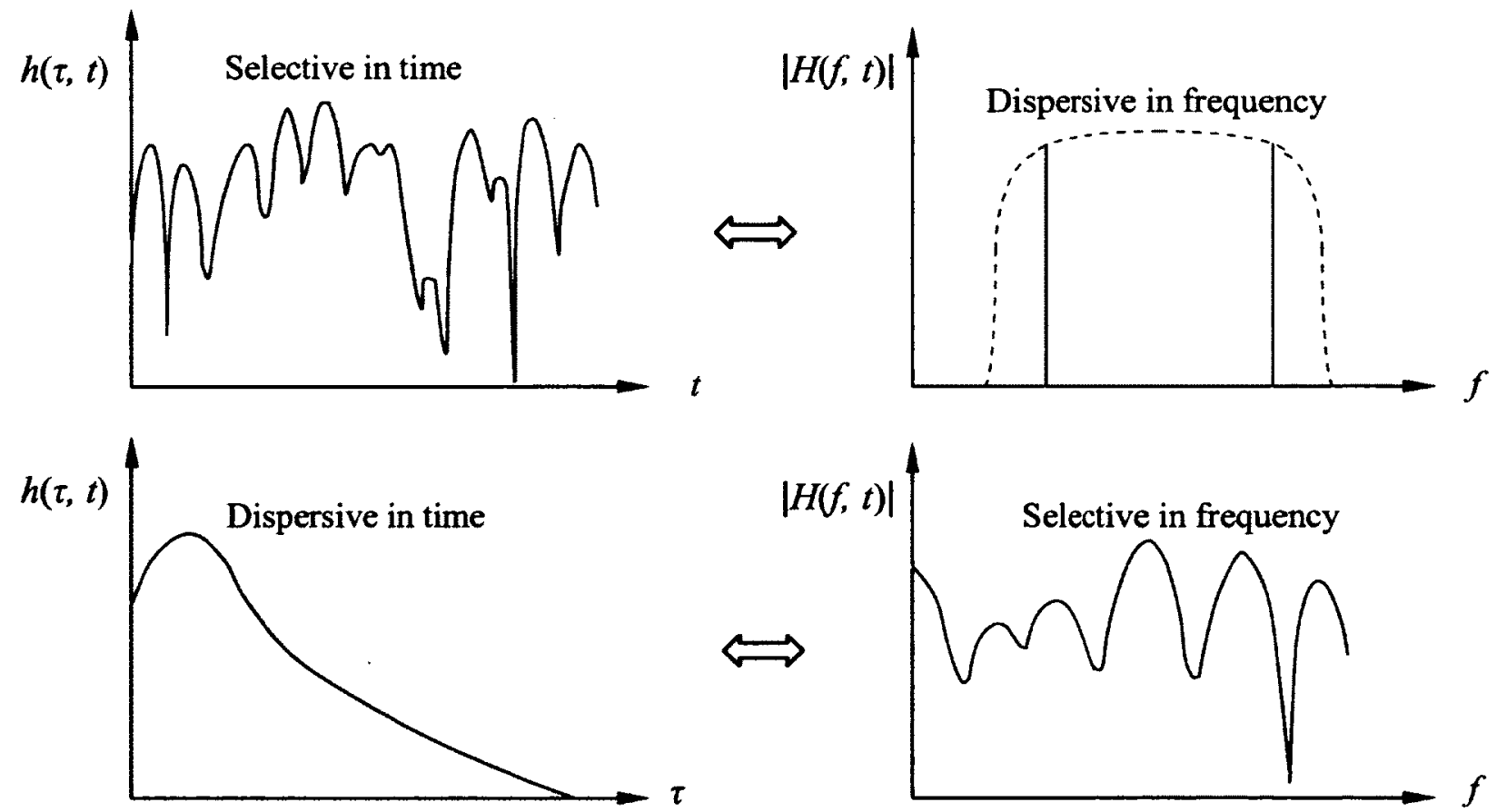

Dispersion in time causes frequency-selectivity, whereas dispersion in frequency causes time-selectivity.

Figure 2.21. Time-Frequency Selectivity and Dispersion Duality 


$$
\begin{aligned}
& \left|f_{1}-f_{2}\right| \leq B_{\mathrm{C}} \text { implies } H\left(f_{1}\right) \simeq H\left(f_{2}\right) \\
& \left|f_{1}-f_{2}\right|>B_{\mathrm{C}} \text { implies } H\left(f_{1}\right) \text { and } H\left(f_{2}\right) \text { are uncorrelated }
\end{aligned}
$$

Coherence bandwidth measures the channel decorrelation in frequency (see Figure 2.20 [31]). The coherence bandwidth is inversely proportional to the root-mean-square (rms) delay spread. By choosing the sub-carrier spacing properly in relation to the channel coherence bandwidth, OFDM can be used to convert a frequency selective channel into a parallel collection of frequency flat sub-carriers.

Time dispersion is a manifestation of multipath propagation that stretches a signal in time so that the duration of the received signal is greater than the transmitted signal as shown in Figure 2.21 [31]. Time dispersion results from the variations of the channel and can be caused by motion of the antenna, which also gives Doppler spread. Fast fading requires short packet durations and thus high bit rates. Time dispersion poses requirements on the synchronization and rate of the convergence of channel estimation. Interleaving may help to avoid burst errors.

Doppler spread $\left(f_{\mathrm{D}}\right)$ is a measure of the spectral broadening caused by the time rate of change of the mobile radio channel, and is defined as the range of frequencies over which the received Doppler spectrum is essentially non-zero. This is shown in Figure 2.20 [31]. The Doppler shift $\left(f_{D \max }\right)$ is given by:

$$
f_{D}=\frac{v \cdot f_{C}}{c}
$$

where $f_{\mathrm{C}}=$ carrier frequency, $v=\max$ speed between $\mathrm{Tx}$ and $\mathrm{Rx}$, and $c=$ speed of light.

Coherence time $\left(T_{\mathrm{C}}\right)$ is a statistical measure of the time duration over which the channel impulse response is considered to be non-varying (See Figure 2.20 [31]). Such channel variation can be significant in wireless communications systems, due to Doppler effects. The coherence time measures the channel decorrelation in time.

$$
T_{c} \simeq \frac{1}{f_{D}}
$$


The coherence time describes the time duration over which the channel response can be considered stable. Signals arriving with a time separation greater than $T_{\mathrm{C}}$ are known as fast fading, are subject to distortion, and are uncorrelated. By contrast, components separated by an amount less than $T c$ observe what is known as a slow fading channel as given in Equation 2.17 .

$$
\begin{aligned}
& \left|t_{1}-t_{2}\right| \leq T_{\mathrm{C}} \text { implies } h\left(t_{1}\right) \simeq h\left(t_{2}\right) \\
& \left|t_{1}-t_{2}\right|>T_{\mathrm{C}} \text { implies } h\left(t_{1}\right) \text { and } h\left(t_{2}\right) \text { are uncorrelated }
\end{aligned}
$$

Frequency dispersion is caused from different insertion phases at different frequencies as shown in Figure $2.21[31]$

Due to the mobility of the mobile terminals, the multipath propagation situation will be continuously changed over time which is described analytically as a time-variant channel impulse response $h(\tau, t)$ or alternatively as a frequency-selective and time-dependent radio channel transfer function $H(f, t)$. For mobility, the Delay Doppler function, $v\left(\tau, f_{D}\right)$ and the Frequency Doppler function, $U\left(f, f_{D}\right)$ can be used as an alternative description of the radio channel behaviour as shown in Figure 2.22.

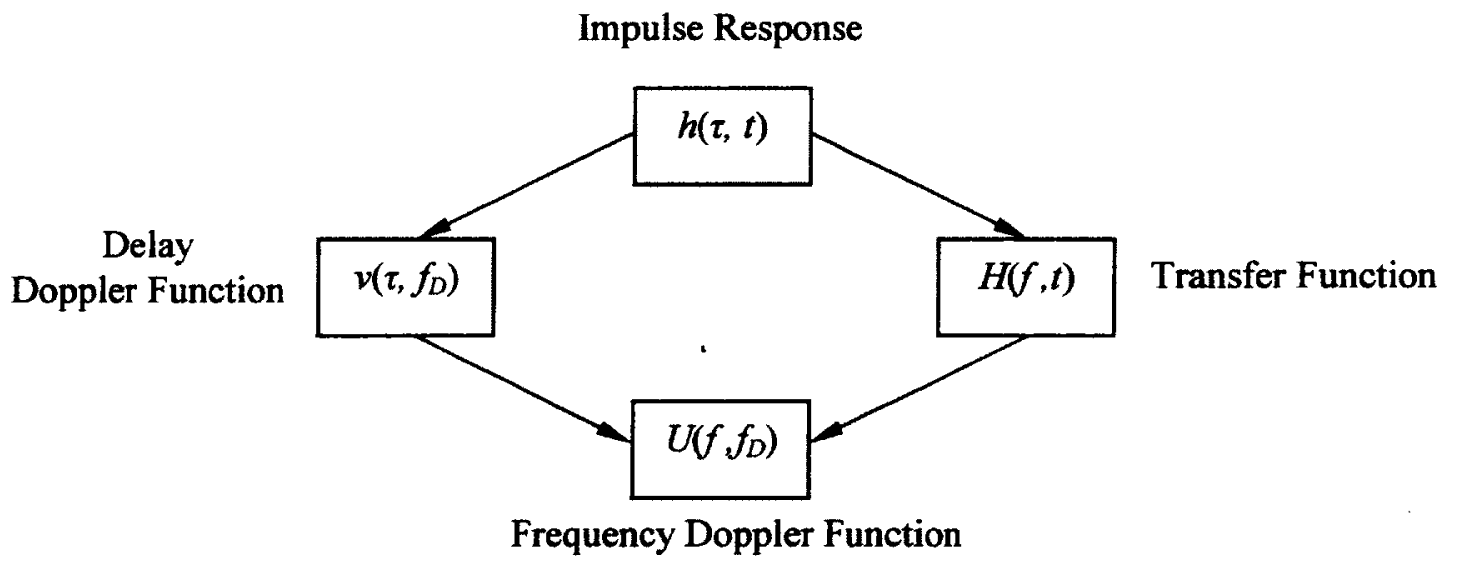

Figure 2.22. Time-Variant Channel Impulse Response and Doppler Functions 
Angular spread $\left(\theta_{\mathrm{RMS}}\right)$ and coherence distance are particularly important in multiple-antenna systems. The coherence distance gives a rule of thumb for how far apart antennas can be spaced in order to be statistically independent. Angular spread at the receiver from all directions (angle-of-arrival - AOA) and at the transmitter (angle-of-departure - AOD) are important parameters that describe the extent of signal spreading in spatial angle. The degree of space selective fading can be charaterized by the coherence distance. The channel is said to be space-selective between two antennas if their separation is larger than the coherence distance. Coherence distance measures the channel decorrelation in space.

Coherence distance $\left(D_{\mathrm{C}}\right)$ is a statistical measure of the maximum spatial separation over which the channel response can be assumed constant. Coherence distance is defined as the spatial displacement when the magnitude of the auto-correlation function remains higher than a threshold. That is, the coherence distance of a multipath channel is the minimum distance between two nearby receiver locations that will receive signals affected quite differently by the channel. This can be related to the behaviour of arrival directions of the reflected radio waves and is characterized by the angular spread of the multiple paths.

$$
D_{C} \simeq \frac{0.2 \lambda}{\theta_{R M S}}
$$

In Space Dispersion, when there is only one line-of-sight (LOS) path, the signal arrives from a single angle. As there are more and more paths, the range of AOA and AOD get broader. Since the power of the received signal fluctuates as the receiver antenna rotates, angle spread results in space-selective fading due to the antenna orientation. In addition, the characteristics of a multipath channel also depend on the location of the transmitter or the receiver. 


\begin{tabular}{|c|c|c|c|}
\hline Fading Parameter & Large & Small & LTE Design Impact \\
\hline $\begin{array}{l}\text { Delay Spread } \\
\left(\tau_{\text {RMS }}\right)\end{array}$ & $\begin{array}{l}\text { If } \tau_{\mathrm{RMS}} \gg T, \text { then } \\
\text { frequency selective. }\end{array}$ & $\begin{array}{l}\text { If } \tau_{\mathrm{RMS}}<<\text {, then } \\
\text { frequency flat. }\end{array}$ & $\begin{array}{l}\text { The larger the delay spread } \\
\text { relative to the symbol time, the } \\
\text { more severe the ISI }\end{array}$ \\
\hline $\begin{array}{l}\text { Coherence } \\
\text { Bandwidth } \\
\left(B_{C}\right)\end{array}$ & $\begin{array}{l}\text { If } \frac{1}{B_{C}} \ll T_{S} \text {, then } \\
\text { frequency flat. }\end{array}$ & $\begin{array}{l}\text { If } \frac{1}{B_{C}} \gg T_{S} \text {, then } \\
\text { frequency selective. }\end{array}$ & $\begin{array}{l}\text { Provides a guideline to } \\
\text { subcarrier with } B_{S C} \approx B_{C} / 10 \text {, and } \\
\text { hence number of subcarriers } \\
\text { needed in OFDM: } L \geq 10 \mathrm{~B}_{\mathrm{SC}} / \mathrm{B}_{\mathrm{c}}\end{array}$ \\
\hline $\begin{array}{l}\text { Doppler Spread } \\
\left(f_{D}=\frac{f_{c} \cdot v}{c}\right)\end{array}$ & $\begin{array}{l}\text { If } f_{\mathrm{c}} \cdot v>>\mathrm{c} \text {, then } \\
\text { fast fading. }\end{array}$ & $\begin{array}{l}\text { If } f_{\mathrm{c}} \cdot v \leq \mathrm{c} \text {, then slow } \\
\text { fading. }\end{array}$ & $\begin{array}{l}\text { As } f_{\mathrm{D}} / B_{S C} \text { becomes non- } \\
\text { negligible, subcarrier } \\
\text { orthogonality is compromised. }\end{array}$ \\
\hline $\begin{array}{l}\text { Coherence Time } \\
\left(T_{C}\right)\end{array}$ & $\begin{array}{l}\text { If } T_{c} \gg T_{S}, \text { then } \\
\text { slow fading. }\end{array}$ & $\begin{array}{l}\text { If } T_{C} \leq T_{S} \text {, then fast } \\
\text { fading. }\end{array}$ & $\begin{array}{l}T_{C} \text { small necessitates frequent } \\
\text { channel estimation and limit the } \\
\text { OFDM symbol duration, but } \\
\text { provides greater time diversity. }\end{array}$ \\
\hline $\begin{array}{l}\text { Angular Spread } \\
\left(\theta_{\mathrm{RMS}}\right)\end{array}$ & $\begin{array}{l}\text { Non-LOS channel, } \\
\text { lots of diversity }\end{array}$ & $\begin{array}{l}\text { Effectively LOS } \\
\text { channel, not much } \\
\text { diversity }\end{array}$ & $\begin{array}{l}\text { Multi-antenna array design, } \\
\text { beamforming vs. diversity }\end{array}$ \\
\hline $\begin{array}{l}\text { Coherence Distance } \\
\left(D_{C}\right)\end{array}$ & $\begin{array}{l}\text { Effectively LOS } \\
\text { channel, not much } \\
\text { diversity. }\end{array}$ & $\begin{array}{l}\text { Non-LOS channel, } \\
\text { lots of diversity. }\end{array}$ & Determines antenna spacing. \\
\hline
\end{tabular}

Table 2.3. Fading Parameters Design Impact on LTE

The Table 2.3 shows a summary of fading parameters and the impact on LTE systems [31]. For instance, by choosing the sub-carrier spacing properly in relation to the channel coherence bandwidth, LTE-OFDM can be used to convert a serial frequency selective fading channel into a parallel collection of frequency flat fading sub-carriers.

In LTE-OFDM digital modulation design, the raised-cosine filter (RCF) is frequently used for pulseshaping and its ability to minimise intersymbol interference (ISI). However, in many practical communications systems, a matched filter is used in the receiver, due to the effects of white noise. That is, a root-raised-cosine filter (RRC), sometimes known as square-root-raised-cosine filter (SRRC), is frequently used as the transmit and receive filter in a digital communication system to perform such matched filtering. 


\begin{tabular}{|c|c|c|c|}
\hline Model & Number of paths & RMS delay spread & Maximum delay \\
\hline Extended Pedestrian A (EPA) & 7 & $45 \mathrm{~ns}$ & $410 \mathrm{~ns}$ \\
\hline Extended Vehicular A (EVA) & 9 & $357 \mathrm{~ns}$ & $2.51 \mu \mathrm{s}$ \\
\hline Extended Typical Urban (EPA) & 9 & $991 \mathrm{~ns}$ & $5 \mu$ \\
\hline
\end{tabular}

Table 2.4. Delay Power Profiles Channel Models in LTE

\begin{tabular}{|c|c|c|c|}
\hline Parameter & $\begin{array}{c}\text { Low Doppler } \\
\text { Frequency }\end{array}$ & $\begin{array}{c}\text { Medium Doppler } \\
\text { Frequency }\end{array}$ & $\begin{array}{c}\text { High Doppler } \\
\text { Frequency }\end{array}$ \\
\hline Frequency & $5 \mathrm{~Hz}$ & $70 \mathrm{~Hz}$ & $300 \mathrm{~Hz}$ \\
\hline Velocity & $\begin{array}{c}2.7 \mathrm{~km} / \mathrm{h} \text { at } 2 \mathrm{GHz} \\
6.4 \mathrm{~km} / \mathrm{h} \text { at } 850 \mathrm{MHz}\end{array}$ & $\begin{array}{c}40.8 \mathrm{~km} / \mathrm{h} \text { at } 2 \mathrm{GHz} \\
88.9 \mathrm{~km} / \mathrm{h} \text { at } 850 \mathrm{MHz}\end{array}$ & $\begin{array}{c}162 \mathrm{~km} / \mathrm{h} \mathrm{at} 2 \mathrm{GHz} \\
381.2 \mathrm{~km} / \mathrm{h} \text { at } 850 \mathrm{MHz}\end{array}$ \\
\hline
\end{tabular}

Table 2.5. Doppler Frequencies and Mobile Velocities in LTE

The 3GPP LTE standard has defined delay profiles for low, medium and high delay spread environments, and the delay profiles are summarized above in Table 2.4 [33]. The three models are defined on a 10ns sampling grid. EVA (Extended Vehicular A) is a propagation channel model based on the International Telecommunication Union (ITU) Vehicular A model, extended to a wider bandwidth of $20 \mathrm{MHz}$. The vehicular channel model represents UE speeds of $30,120 \mathrm{~km} / \mathrm{h}$ and higher, while the Extended Pedestrian A (EPA), represents a UE speed of $3 \mathrm{~km} / \mathrm{h}$, and Extended Typical Urban (ETU) is a propagation channel model based on the GSM Typical Urban model, extended to a wider bandwidth of $20 \mathrm{MHz}$. It models a scattering environment which is considered to be typical in a urban area.

The classical Doppler spectrum with uniformly distributed angles of arrival of the paths is applied in the LTE channel models. The classical Doppler spectrum is also referred to as Clark's spectrum or Jake's spectrum. The classical Doppler spectrum is characterized by the maximum Doppler frequency. Three typical maximum Doppler frequencies are specified for the LTE channel models. as shown above in Table 2.5 [33]. 


\subsubsection{Channel Estimation}

In OFDM systems, efficient channel estimation schemes are essential for coherent detection of a received signal. Channel estimation is the task of estimating the frequency response of the radio channel the transmitted signal travels before reaching the receiver antenna.

The channel estimation can be performed using the time-domain approach and frequency-domain approach, In the time-domain approach, the channel impulse response (CIR) is estimated before FFT processing of the training symbols. In the frequency-domain approach, the channel frequency response (CFR) is estimated by using two repeat training symbols.

After multi-carrier demodulation, the received signal is typically correlated in two dimensions, in time and frequency. By periodically inserting pilots in the time-frequency grid to satisfy the $2 \mathrm{D}$ sampling theorem, the channel response can be reconstructed by exploiting its correlation in time and frequency. Channel estimation provides the distortion information of the transmission signal when it propagates through the channel to the equalizers so that fading effects and/or co-channel interference can be removed and the original transmitted signal can be restored. The receiver signal detector, such as a MLSE or MAP, needs to know channel impulse response (CIR) characteristics to ensure successful equalisation (removal of ISI). The channel estimator is able to estimate the CIR by exploiting the known transmitted bits and the corresponding received samples. It is noted that equalization without separate channel estimation (e.g., with linear, decision-feedback, blind equalizers) is also possible.

For coherent detection the channel transfer function must be estimated. For such an estimation to take place, pilot symbols are used in the OFDM time-frequency two-dimensional grid. In applications where the channel changes very slowly in time, a one-dimensional channel estimation can be used. Coherent modulation allows arbitrary signal constellations, but efficient channel estimation strategies are required for coherent detection and decoding. There are two ways to transmit training symbols: with a preamble or 
with pilot tones. Preambles entail sending a certain number of training symbols prior to the user data symbols. In the case of OFDM, one or two preamble OFDM symbols are typical. Pilot tones involve inserting a few pilots among the subcarriers. Channel estimation in OFDM systems can be performed in a variety of ways, but it is typical to use a preamble for synchronization and initial channel estimation and pilot tones for tracking the time-varying channel in order to maintain accurate channel estimates.

Differentially coherent detection eliminates the need for channel estimation, relying instead on the assumption that the channel response changes slowly, either between carriers (differential encoding/detection in frequency) or between blocks (differential encoding/detection in time). Differential detection eliminates the overhead of pilot-assisted channel estimation. Noncoherent detection does not need any channel knowledge or estimation, it is applicable even in most degraded and fast fading channels, making it much more attractive than coherent detection under unfavourable channel conditions.

The Channel estimation procedure is as follows. The first step is to calculate the inverse DFT of the input frequency-domain sequence to get the input to time-domain $x(n)=\operatorname{IDFT}\{X(k)\}$, where $n=0,1,2, \ldots, N-1$. The second step is the insertion of the guard interval: $x_{f}(n)=\left\{\begin{array}{cc}x(N+n) & n=-N_{g},-N_{g}+1, \ldots,-1 \\ x(n) & n=0,1, \ldots, N-1\end{array}\right.$. The third step is to perform a circular convolution with the channel and the addition with the additive white Gaussian noise $y(n)=x(n) \otimes y(n)+w(n)$. The fourth step is the removal of the guard interval or cyclic prefix $y(n)=y_{\mathcal{A}}(n)$, where $n=0,1, \ldots, N-1$. The fifth step is to calculate the DFT of the time-domain output signal in frequency-domain $Y(k)=D F T\{y(n)\}$, where $k=0,1, \ldots, N-1$. The sixth step is to compute the frequency-domain output sequence $Y(k)=X(k) \cdot H(k)+I(k)+W(k)$, where $k=0,1, \ldots, N-1$. The seventh and final step is to calculate an estimate of the channel by taking the ratio of $Y(k)$ by the channel transfer function $H_{\mathrm{e}}(k), X_{e}(k)=\frac{Y(k)}{H_{e}(k)}$, where $k=0,1, \ldots, N-1$. 


\subsection{Spectrum-Agile Radios}

A "Cognitive Radio" is a radio that can change its transmitter parameters based on interaction with the environment in which it operates (FCC). Figure 2.23 [26] shows a suggested functional block diagram of cognitive radio system architecture. It is comprised of a wideband antenna: omnidirectional for the spectrum sensing and directional for the cognitive link, a frequency-agile RF frontend block with wideband and reconfigurable features, a dual-stage wideband spectrum sensing block, a physical (PHY) layer block, and a Medium Access Control (MAC) layer block.

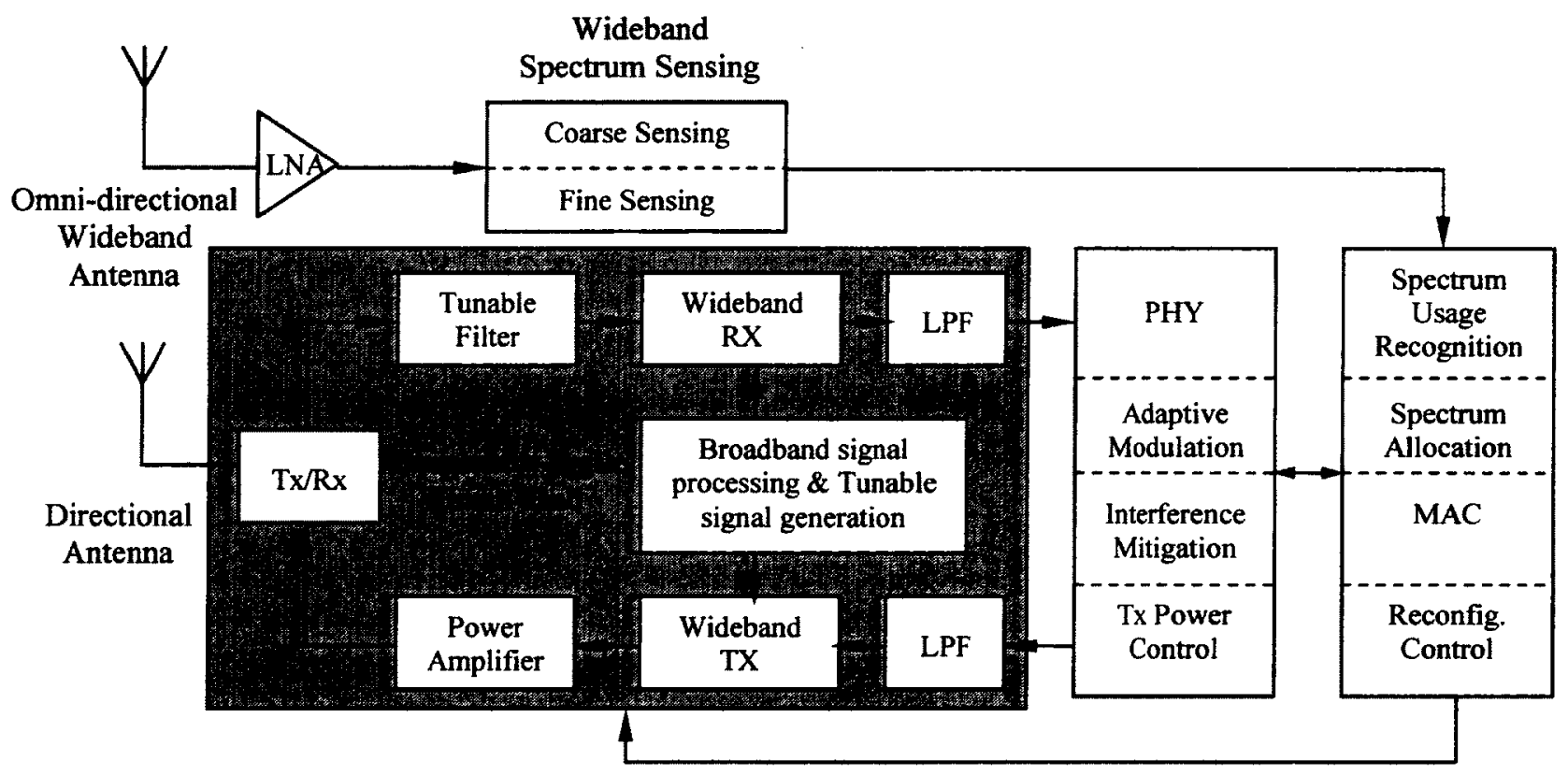

Figure 2.23. Frequency-Agile Transceiver Concept Block Diagram

The basic block diagram of a frequency-agile radio system above consists of two functional blocks: a PHY (Physical Layer) block where the digital signal processing is performed as well as the signal generation and analysis tasks of the system, and a MAC (Medium Access Control Layer) block which determines the spectrum available as well as the spectrum to be utilized.

The following sections will discuss in more details the main attributes and characteristics of frequency-agile radios applied in the LTE/LTE-Advanced context. 


\subsubsection{Dynamic Frequency Selection (DFS)}

Originally used to describe a technique to avoid radar signals by 802.11 a networks which operate in the 5 $\mathrm{GHz}$ U-NII band, this concept is generalized to refer to an automatic frequency selection process intended to achieve some specific objective (like avoiding harmful interference to a radio system with a higher regulatory priority). Dynamic Frequency Selection (DFS) describes the technique where prior to transmitting, a radio attempts to detect the presence of other, possibly licensed, radios and avoids operating on frequencies that could cause interference with other radios or other systems. DFS can be defined as a general term used to describe mitigation techniques that allow detection and avoidance of cochannel interference with other radios in the same system or with respect to other systems.

\subsubsection{Transmit Power Control (TPC)}

Originally a mechanism for 802.11 a networks to lower aggregate transmit power by $3 \mathrm{~dB}$ from the maximum regulatory limit to protect ES-SCH (Earth Exploration Satellite Systems) operations, this concept is now generalized as a mechanism that adaptively sets the transmitter power based on the spectrum or regulatory environment.

\subsubsection{Dynamic Spectrum Access (DSA)}

Dynamic Spectrum Access (DSA) is a technique for cognitive radio in 802.22 networks as shown in Figure 2.24. DSA is defined as the real-time adjustment of spectrum utilization in response to changing circumstances and objectives. Cognitive radio is a type of radio in which communication systems are aware of their environment and internal state and can make decisions about their radio operating behaviour based on that information and predefined objectives. Dynamic Spectrum Access (DSA) is the extension of DFS to include a scenario where the radio (Cognitive Radio) actively seeks free spectrum bands in order to enhance the services by having access to a larger share of the spectrum bandwidth. 


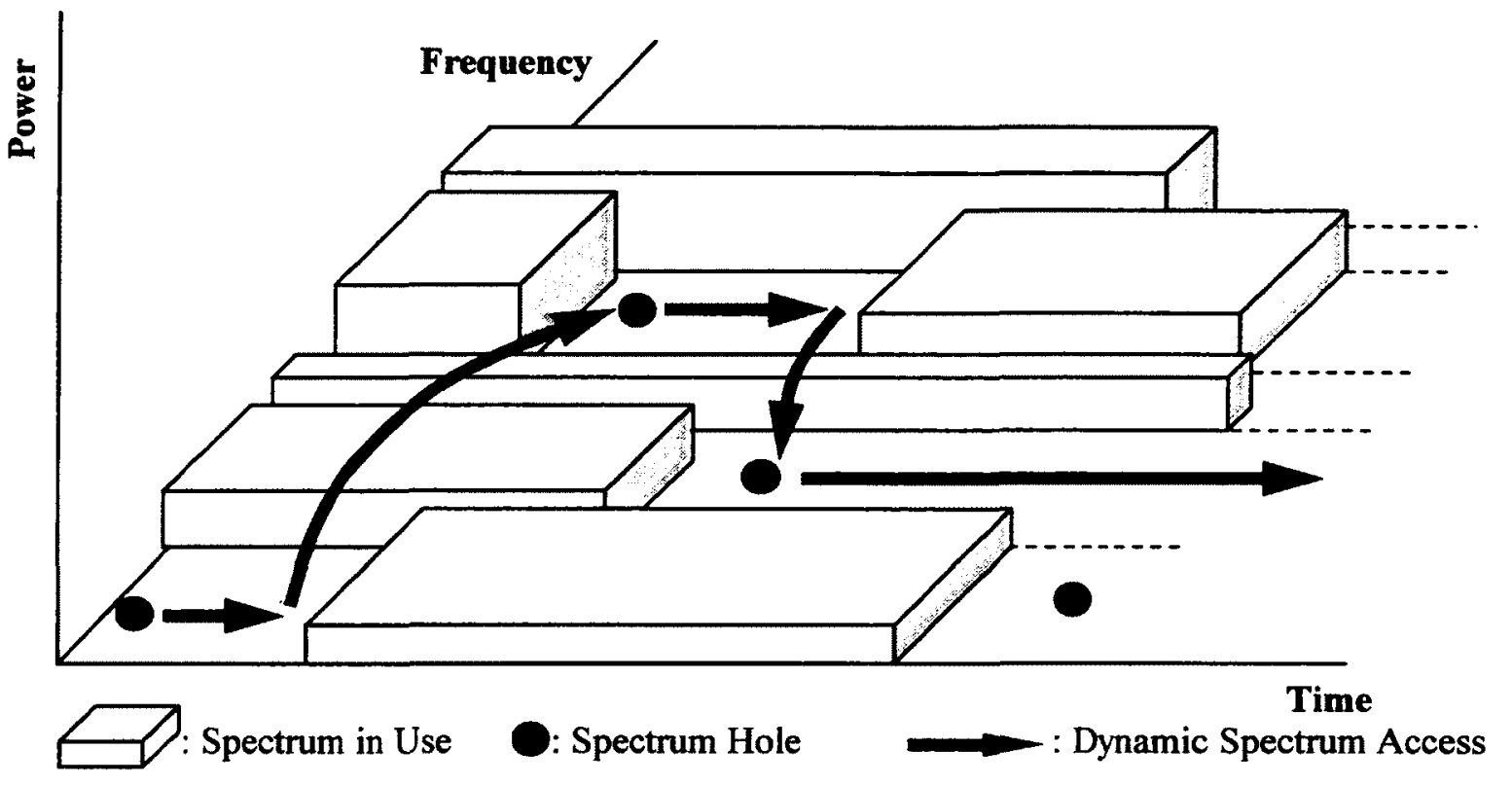

Figure 2.24. Spectrum Hole and Dynamic Spectrum Access (DSA)

To some extent, the application of CR/DSA techniques can be thought of as an evolution of coexistence techniques of DFS/TPC. The IEEE 802.22 standard (July 2011) specifies the air interface, including the cognitive medium access control layer (MAC) and physical layer (PHY), of point-to-multipoint wireless regional area networks comprised of a fixed base station with fixed and portable user terminals operating in the VHF/UHF TV broadcast bands between $54 \mathrm{MHz}$ to $862 \mathrm{MHz}$. Since it is not always possible to have paired TV channels available, IEEE 802.22 is initially defining a single time-domain duplex (TDD) mode, with plans to define a frequency-division duplex (FDD) mode as a future amendment to the standard.

\subsubsection{Self Organizing Network (SON)}

The LTE specification inherently supports SON features like Automatic Neighbour Relation (ANR) detection, which is the 3GPP LTE Rel. 8 flagship feature. Newly added base stations should be selfconfigured in line with a 'plug-and-play' paradigm, while all operational base stations will regularly selfoptimize their parameters and algorithmic behaviour in response to observed network performance and radio conditions. Furthermore, self-healing mechanisms can be triggered to temporarily compensate for a detected equipment outage, while awaiting a more permanent solution. 


\subsubsection{Coexistence and Self-Coexistence Mechanism}

Coexistence is defined as a state of acceptable co-channel and/or adjacent channel operation of two or more radio systems (possibly using different wireless access technologies) within the same geographical area; whereas self-coexsistence is the coexistence with other systems of the same type. In a typical deployment scenario, multiple 802.22 systems may operate in the same vicinity. Mutual interference among these collocated WRAN systems due to co-channel operation could degrade the system performance significantly. To address this issue, the 802.22 MAC specifies a self-coexistence mechanism based on the coexistence beacon protocol (CBP) and consisting of spectrum sharing schemes that address different coexistence needs in a coherent manner. Two unlicensed wireless networks are said to coexist if they can operate in the same location without causing significant interference to one another.

\subsubsection{Ranging and Geolocation (GEOLOC)}

Ranging is a process or method to determine the distance from one location or position to another location or position. To deal with the large propagation delays and varying RF signal quality between CPEs and the $\mathrm{BS}$, the MAC incorporates a ranging procedure. Ranging is a collection of processes by which the CPE and BS maintain synchronization as well as quality of the RF communication link between them. Distinct processes are used for managing downstream and upstream.

Geolocation is the process of acquiring the location data of a device, determining its latitude and longitude, and producing the corresponding NMEA (National Marine Electronics Association) string. 


\subsubsection{Vertical Handover (VHO)/Media Independent Handover (MTH)}

Vertical handover or vertical handoff refers to a network node changing the type of connectivity it uses to access a supporting infrastructure, usually to support node mobility. For example, a suitably equipped mobile smartphone might be able to use both a high-speed wireless LAN and a cellular technology for Internet access. Wireless LAN connections generally provide higher speeds, while cellular technologies generally provide more ubiquitous coverage. Thus, the mobile smartphone user might want to use a wireless LAN connection whenever one is available, and to 'fall over' to a cellular connection when the wireless LAN is unavailable. Vertical handovers refer to the automatic fallover from one technology to another in order to maintain communication. This is different from a 'horizontal handover' between different wireless access points that use the same technology in that a vertical handover involves changing the data link layer technology used to access the network. Media Independent Handover (MIH) is a standard being developed by IEEE 802.21 to enable the handover of IP sessions from one layer 2 access technology to another, to achieve mobility of end user devices.

\subsubsection{Carrier Aggregation (CA)}

The concept of spectrum aggregation is to exploit spectrum fragments simultaneously to create wider bandwidths for communications systems. To meet LTE-Advanced Release 10 requirements, support of wider transmission bandwidths is required than the $20 \mathrm{MHz}$ bandwidth (specified in 3GPP Release 8/9). The preferred solution to this is carrier aggregation.

Carrier aggregation is of the most distinct features of $4 \mathrm{G}$ LTE-Advanced and allows expansion of effective bandwidth delivered to a user terminal through concurrent utilization of radio resources across multiple carriers. Multiple component carriers are aggregated to form a larger overall transmission bandwidth. Carrier aggregation (CA) is one of the main features of LTE-Advanced in Rel-10 for meeting the peak data rate requirements of IMT-Advanced; $1 \mathrm{~Gb} / \mathrm{s}$ and $500 \mathrm{Mb} / \mathrm{s}$ for the downlink and uplink, respectively. 


\subsubsection{Adaptive Bit Loading (ABL)}

Adaptive bit loading is used to enhance the performance of multicarrier transceivers by tailoring the subcarrier signal constellations to the channel conditions, which can vary across the subcarriers. The three main attributes of adaptive bit loading are: seeking water filling capacity, adaptation to channel fading, and adaptation to interference.

\subsubsection{Adaptive Coding and Modulation (ACM)}

$\mathrm{ACM}$ is not a new concept. It has been used for some time in wireless communications, including terrestrial microwave applications, and more recently over satellite links. Its primary function is to optimize throughput in a wireless data link, by adapting the modulation order used and the Forward Error Correction code rate which both directly affect spectral efficiency, according to the noise conditions (or other impairments) on the link.

In ACM, users with better SNR (closer to the BS) get higher order modulation, those farther from the BS get lower order modulation, ensuring the best performance for each user within the BS coverage as shown in Figure 2.25.

LTE uses adaptive coding and modulation to improve data throughput. This technique varies the downlink modulation-coding scheme based on the channel conditions for each user. When the link quality is good, the LTE system uses a higher order modulation scheme, which results in more system capacity. On the other hand, when link conditions are poor due to problems such as signal fading, the LTE system can change to a lower modulation scheme to maintain reliability and minimize error (see Figure 2.26). 


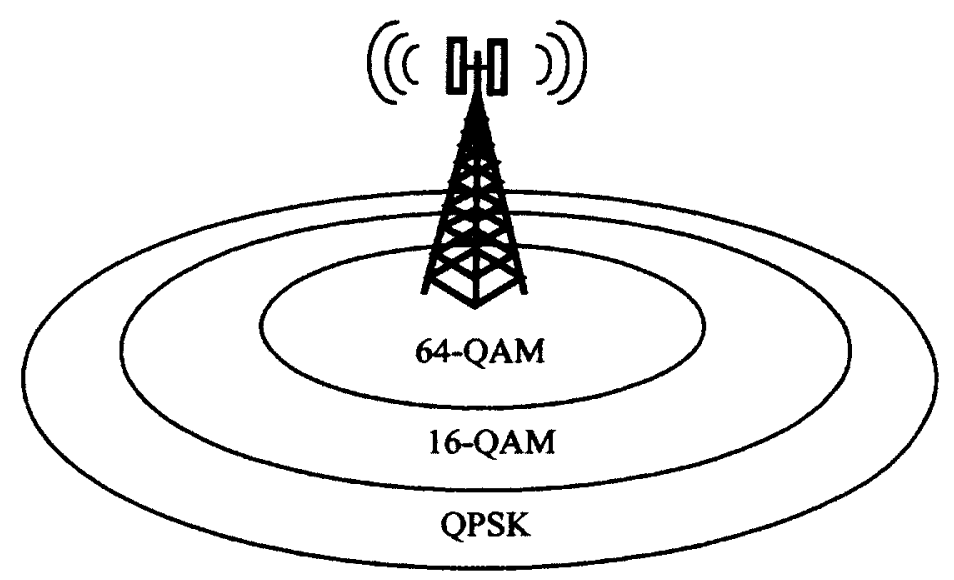

Figure 2.25. LTE Adaptive Coding Modulation (ACM)

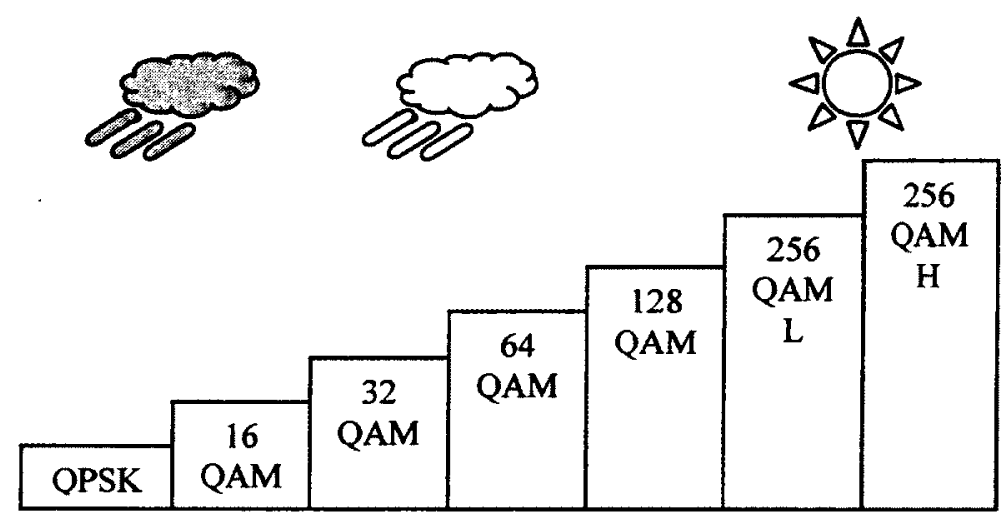

Figure 2.26. ACM Modulation Mode

\subsubsection{Heterogeneous Networks (HetNets)}

Heterogeneous networks (HetNets) refers to the coexistence between access nodes with different RF characteristics and coverage area, potentially operating over the same set of frequency bands and using the same technology. For instance, macro and picocells using dedicated lines for backhaul and open to all subscribers may coexist with femtocells deployed by individuals, the latter employing home-use broadband access technologies for the backhaul and having more restricted association policies. Operators can also deploy relay nodes to support multi-hop communications and extend the coverage area. HetNets are meant to address the expected explosion in demand for high data rate services and the consequent need for substantially higher spectral efficiency. They also present new challenges that seem tailor-made for the capabilities of cognitive radios. 


\subsection{Conclusion}

In summary, LTE, OFDM, and MIMO have been introduced and reviewed. Interferences and uncertainties in OFDM have been described. Mobile Radio Channel Characterizations have been discussed. The mobile radio channel can be modeled and characterized by two important system parameters: the maximum multipath delay $\tau_{\max }$ and the maximum Doppler frequency $f_{D \max }$ which are transferred into the coherence time $T_{C}$ and the coherence bandwidth $B_{C}$ of the radio channel. Delay spread leads to inter-symbol interference and frequency-selective fading. Doppler spread leads to time variations of the channel, and broadening of signals bandwidth. Since the signal bandwidth is smaller than the bandwidth of the channel impulse response (alternatively $B_{\mathrm{S}}<B_{\mathrm{C}}$ ) in a flat fading channel, it is often referred to as a narrowband channel. When the signal bandwidth is larger than the bandwidth of channel impulse response $\left(B_{\mathrm{S}}>B_{\mathrm{C}}\right)$ frequency-selective fading occurs, and the channel is often referred to as a wideband channel.

While the power-delay profile gives the statistical power distribution of the channel over time for a signal transmitted for only an instant, the Doppler power spectrum gives the statistical power distribution of the channel over frequency for a signal transmitted at one exact frequency. While the power-delay profile was caused by multipath between the transmitter and the receiver, the Doppler power spectrum is caused by motion between the transmitter and the receiver.

LTE is aimed primarily at low mobility applications in the 0 to $15 \mathrm{~km} / \mathrm{h}$ range, where the highest performance will be achieved. The system is capable of working at higher speeds, and will be supported with high performance from 15 to $120 \mathrm{~km} / \mathrm{h}$ and functional support from 120 to $350 \mathrm{~km} / \mathrm{h}$. Support for speeds of 350 to $500 \mathrm{~km} / \mathrm{h}$ is under consideration. LTE-FDD and LTE-TDD applications as well as the advantages and drawbacks have been discussed and analyzed. The frequency-agile radio concept and attributes have also been presented. 


\section{Chapter 3 . Spectrum Sensing Techniques}

\subsection{Introduction}

The main topic of this chapter is a discussion of several major spectrum sensing techniques. Spectrum sensing is the first step towards the implementation of a frequency-agile radio system. There are three major categories of spectrum sensing techniques; primary transmitter detection, cooperative detection and interference-based detection. Based on the locally perceived primary signal power level, a point on the plane can be marked as either a black space, grey space, or white space. The black spaces refer to spectra occupied by high power primary signals, while the grey spaces refer to spectra occupied by low power primary signals. White spaces refer to the unused frequency voids across time or space.

Spectrum sensing is the detection of a Primary User (PU) in a frequency band of interest which in turn enables the Secondary User (SU) or the Cognitive Radio (CR) user to make a decision about spectrum usage. Primary users are legacy spectrum license holders and secondary users are non-license users who access the licensed spectrum dynamically.

Detection is the process of extracting the symbols from the signal waveforms. Demodulation is the process of removing the carrier to obtain the original signal waveform. Coherent detection is the process of demodulating a signal with a local carrier of same frequency and phase (requiring a complex receiver but providing better error/higher SNR performance), whereas non-coherent detection requires no reference wave (requiring a less complex receiver but providing worse error/lower SNR performance). Differential detection uses the previous symbol for the reference signal. Coherent demodulation requires channel state information (CSI). The CSI is estimated using transmitted pilot (training) signals. Noncoherent demodulation does not require CSI and no training is required. Coherent reception provides better performance than differential, but requires a more complex receiver. 


\subsection{Detection Theory}

Noise is the unwanted energy that interferes with the ability of the receiver to detect the desired signal. In fact, noise determines how small a signal a receiver can detect, whereas linearity determines how large a signal a receiver can recieve (nonlinearity clips the signal). Noise power $P_{N}$ is expressed in terms of the temperature $T_{o}$ of a matched resistor at the input of the receiver and is defined as:

$$
P_{N}=k \cdot T_{0} \cdot B \quad \mathrm{~W}
$$

where

$k=$ Boltzmann's Constant $=1.38 \times 10^{-23} \mathrm{Joule} /{ }^{\circ} \mathrm{K} ; \quad B=$ Receiver Bandwidth $(\mathrm{Hz})$

$T_{o}=$ Absolute temperature of the receiver input $\left({ }^{\circ} \mathrm{K}\right)=290^{\circ} \mathrm{K}$

$P_{N}=-114 \mathrm{dBm}$ for a $1 \mathrm{MHz}$ bandwidth; $\quad P_{N}=-174 \mathrm{dBm}$ for a $1 \mathrm{~Hz}$ bandwidth

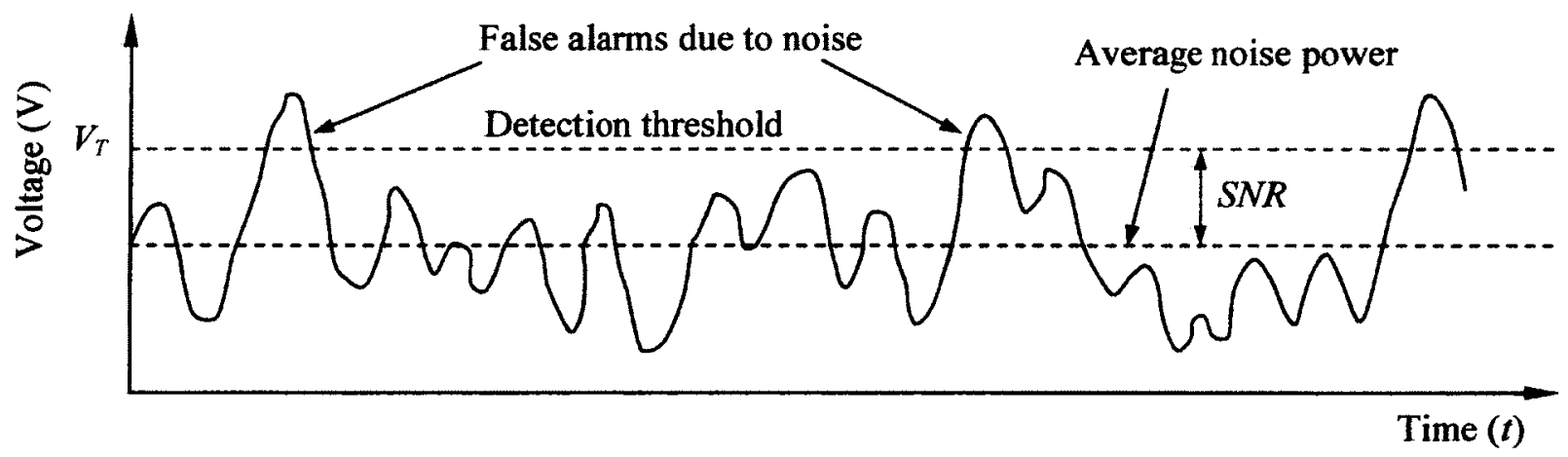

Figure 3.1. Detection of Signals in Noise

Figure 3.1 depicts the concept of required signal-to-noise ratio $S / N$. It can be seen that if the noise alone exceeds the $S N R$ ratio, this indicates a false alarm.

Sensitivity in a receiver is normally taken as the minimum input signal $\left(\mathrm{S}_{\min }\right)$ required to produce a specified output signal having a specified signal-to-noise (SNR) ratio and is defined as the minimum signal-to-noise ratio times the mean noise power. For a signal impinging on the antenna, system level sensitivity, known as minimum operational sensitivity (MOS), is the minimum input signal divided by the antenna gain, i.e. $\mathrm{S}_{\min } / G$. Including the effect of the receiver noise figure (NF) yields:

$$
\mathrm{S}_{\min }=S N R \cdot k \cdot T_{0} \cdot B \cdot N F
$$

The sensitivity is also known as MDS (minimum detectable signal). 
The energy detector is a blind detector that does not rely on features of a specific signal type. This sensing technique is a simple method for quickly determining if a signal is present in the channel, except for the case of weak signals. As a result, this is a coarse sensing technique.

The LTE sampled signal $y(n)$ defined as:

$$
y(n)=x(n)+w(n)
$$

where $x(n)$ is the signal component and $w(n)$ is the noise component. The power of $x(n)$ is $P_{S}$ and the power of $w(n)$ is $P_{N}$. The test statistic for the energy sensing technique is given by:

$$
T=\frac{1}{M} \sum_{n=1}^{M} y(n) \cdot y^{*}(n)
$$

Then probability of false alarm $P_{F A}$ is defined as :

$$
P_{F A}=P\left(T>\gamma \mid H_{0}\right)
$$

The probability of detection $P_{D}$ is defined as:

$$
P_{D}=P\left(T>\gamma \mid H_{1}\right)
$$

where $\gamma$ is the detection threshold, $H_{0}$ and $H_{1}$ represent the situations where the signal is absent and is present respectively. The probability of misdetection $P_{M D}$ is defined as:

$$
P_{M D}=1-P_{D}
$$

For probability of cooperative detection $\left(P_{C D}\right)$ and probability of cooperative false alarm $\left(P_{C F A}\right)$ we have:

$$
\begin{gathered}
P_{C D}=1-\prod_{i=0}^{N-1}\left(1-P_{D, i}\right) \\
P_{C F A}=1-\prod_{i=0}^{N-1}\left(1-P_{F A, i}\right)
\end{gathered}
$$

where $i$ is the sensor index, and $N$ is the number of sensors (see Section 3.2). The spectrum sensing technique can be represented as a threshold comparison. If $T>\gamma$, then the channel is classified as occupied and if $T \leq \gamma$. then the channel is classified as vacant. The probability of detection depends on the signal power. 
As an example, the requirements imposed by the WRAN IEEE 802.22 standard [13] are $P_{f a}<0.1$ and $\boldsymbol{P}_{m d}$ $<0.1$. The Q-function is the integral of the tail probability of the standard normal distribution. $Q(x)$ is given by:

$$
Q(z)=p(X \geq z)=\frac{1}{\sqrt{2 \pi}} \int_{z}^{\infty} e^{-\frac{x^{2}}{2}} d x
$$

The probability that a random variable deviates by a given amount from its expectation is referred to as a tail probability. Since Matlab does not have a built-in $Q(x)$ function, we use its erf function instead:

$$
\operatorname{erf}(x)=\frac{2}{\sqrt{\pi}} \int_{0}^{x} e^{-t^{2}} d t
$$

Also the complementary error function $\operatorname{erfc}(x)$ which corresponds to the $\operatorname{erf}(x)$, is defined as:

$$
\operatorname{erfc}(x)=\frac{1}{\sqrt{2 \pi}} \int_{x}^{+\infty} e^{-t^{2}} d t=1-\operatorname{erf}(x) ; \quad Q(x)=\frac{1}{2} \operatorname{erfc}\left(\frac{x}{\sqrt{2}}\right)
$$

The relationship between $Q(x)$ and $\operatorname{er} f(x)$ is:

$$
Q(x)=\frac{1}{2}-\frac{1}{2} \operatorname{erf}\left(\frac{x}{\sqrt{2}}\right) ; \quad \operatorname{erf}(x)=1-2 Q(\sqrt{2} x)
$$

Figure 3.2 shows the Probability of Occurrence (POC) curves that illustrate the idea of spectrum sensing with probability density functions of noise, and of signal plus noise. The double hatched area represents the probability of false alarm $P_{F A}$, the shaded area and double hatched area represents probability of correct detection $\boldsymbol{P}_{D}$, the dotted area represents the probability of a misdetection $\boldsymbol{P}_{M D}$.

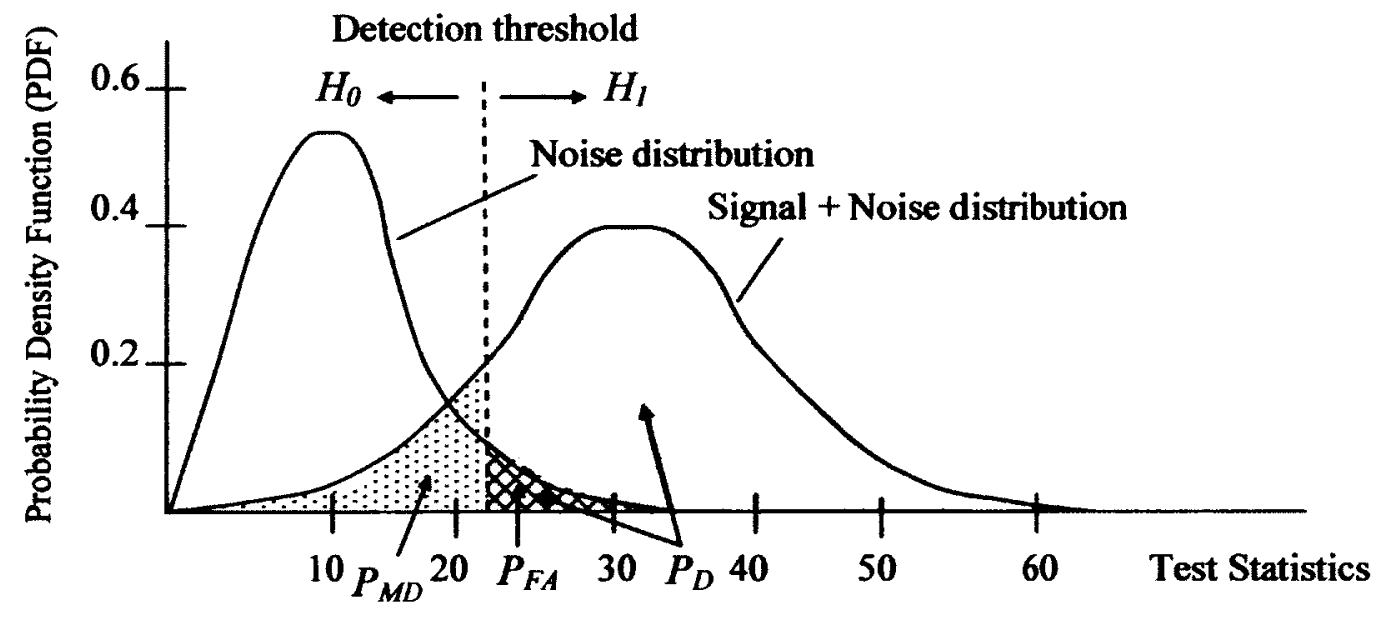

Figure 3.2. Probability of Occurrence (POC) $P_{D}$ and $P_{F A}$ Curves 
The Neyman-Pearson criterion is motivated by the goal of obtaining the best possible detection performance while guaranteeing that the false alarm probability does not exceed some tolerable value.

The Likelihood Ratio Test (LRT) test is as ubiquitous in detection theory and statistical hypothesis testing as is the Fourier transform in signal filtering and analysis. LRT is based on Neyman-Pearson theorem and is given by:

$$
\frac{p_{y}\left(y \mid H_{1}\right)}{p_{y}\left(y \mid H_{0}\right)} \underset{H_{0}}{\stackrel{H_{1}}{\gtrless}}-\gamma
$$

To find the optimum solution, we need to maximize $F=P_{D}+\gamma\left(P_{F f}-\alpha\right)$ and then choose $\gamma$ to satisfy the constraint $P_{F A}=\alpha$, where $\gamma$ is the detection threshold, $\alpha$ is the maximum allowable false alarm probability. In detection theory and spectrum sensing, the most widely used distributions are the Gamma and the ChiSquare distribution. These functions are important mathematics for hypothesis testing, and constructing confidence intervals. The Gamma function $\Gamma$ is defined as:

$$
\Gamma(n)=\int_{0}^{\infty} t^{n-1} e^{-t} d t \quad n>0
$$

This integral is positive for $0<n$, because the integrand id positive. Values of it are often given in a table of integrals. If $n>1$, integration of gamma function of $n$ by parts yields

$$
\Gamma(t)=\left[-t^{n-1} e^{-y}\right]_{0}^{\infty}+\int_{0}^{\infty}(n-1) t^{n-2} e^{-t} d t=(n-1) \int_{0}^{\infty} t^{n-2} e^{-t} d t=(n-1) \Gamma(n-1)
$$

Thus when $n$ is a positive integer, we have that $\Gamma(n)=(n-1) !$; and, for this reason, the gamma is called the generalized factorial. As a matter of fact, $\Gamma(1)$ corresponds to 0 !, and it is noted that $\Gamma(1)=1$. An alternative notation which was originally introduced by Gauss and which was sometimes used is the Pi function, which in terms of the gamma function is:

$$
\Pi(x)=\Gamma(x+1)=x \Gamma(x)
$$

The random variable $\mathrm{x}$ has a gamma distribution if its PDF is defined by:

$$
f(x)=\frac{1}{\Gamma(\alpha) \theta^{\alpha}} x^{\alpha-1} e^{-x / \theta}, \quad 0 \leq x<\infty
$$


Let $X$ have a gamma distribution with $\theta=2$ and $\alpha=r / 2$, where $r$ is a positive integer.

$$
\chi^{2}(r)=f(x)=\frac{1}{\Gamma(r / 2) 2^{r / 2}} x^{r / 2-1} e^{-x / 2}, \quad 0 \leq x<\infty
$$

If the above equation is the PDF of $X$, then $X$ has chi-square distribution with $r$ degrees of freedom, which denotes as $\chi^{2}(r)$. The mean and variance of the chi-square distribution are:

$$
\begin{gathered}
\mu=\alpha \theta=\left(\frac{r}{2}\right) 2=r \\
\sigma^{2}=\alpha \theta^{2}=\left(\frac{r}{2}\right) 2^{2}=2 r
\end{gathered}
$$

In fact, the Chi-squared distribution is a special case of the Gamma distribution.

Figure 3.3 illustrates the probability of detection $P_{D}$ and probability of false alarm $P_{F A}$ of a primary user (PU). The sensing performance is improved as the number of degree-of-freedom increases.

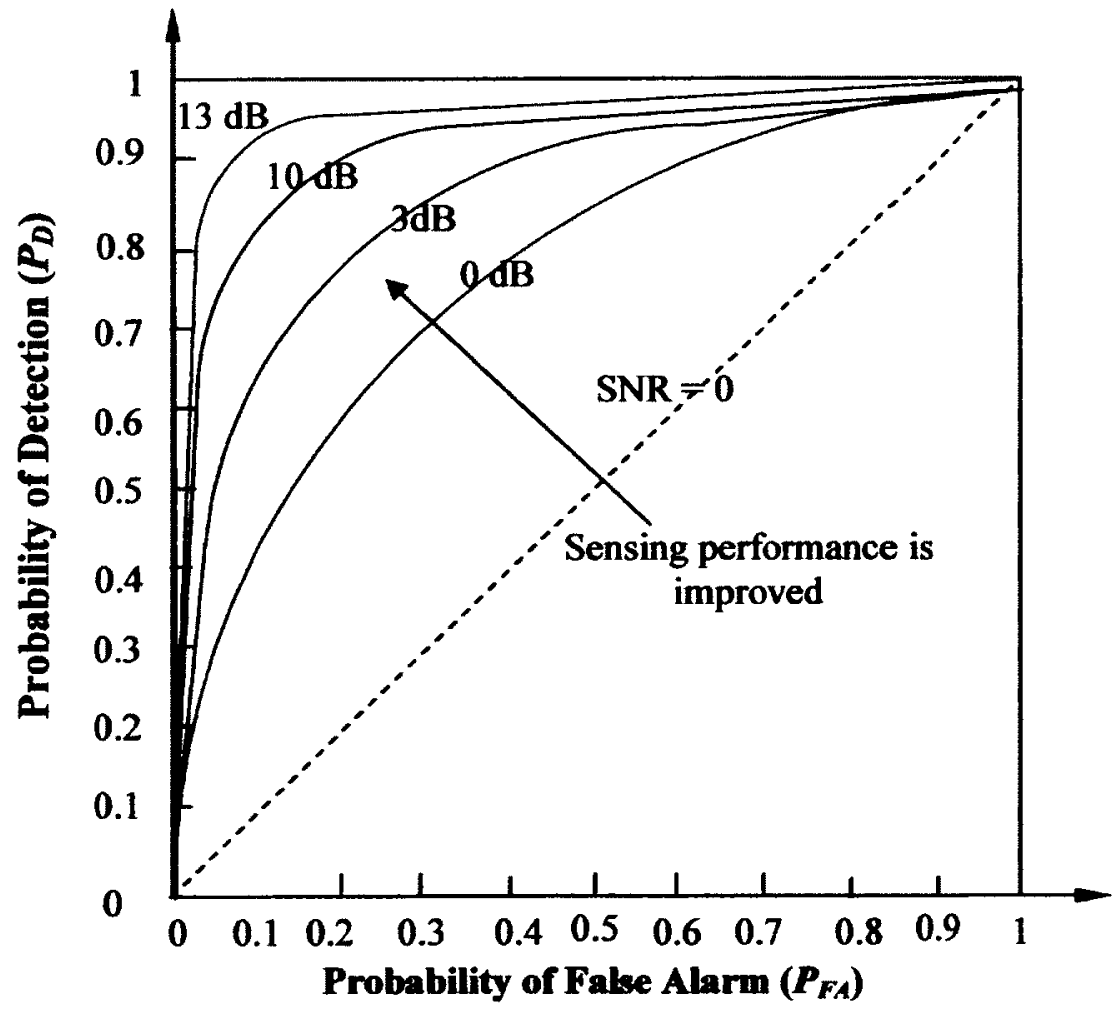

Figure 3.3. Receiver Operating Characteristics (ROC) $\boldsymbol{P}_{D}$ and $\boldsymbol{P}_{F A}$ Curves 
Although the proportion between the false alarm probability and the detection probability can be adjusted via threshold control, it is not possible to simultaneously attain a low false alarm probability and a high detection probability or a low miss detection probability, as long as the probabilistic characteristics of the received signal are fixed.

Figure 3.4 shows that in order to enhance the sensing performance, the distance between two PDFs needs to be lengthened (Fig. 3.4(a)) by increasing the system SNR or the PDF of each hypothesis should be steepened (Fig. 3.4(b)) by reducing the noise power (i.e., reduce the variance).

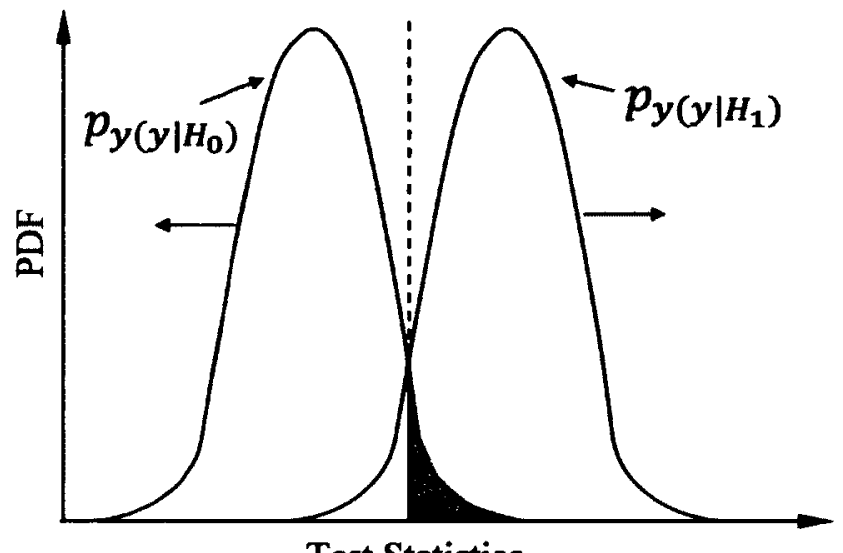

Test Statistics

(a) Increase System SNR

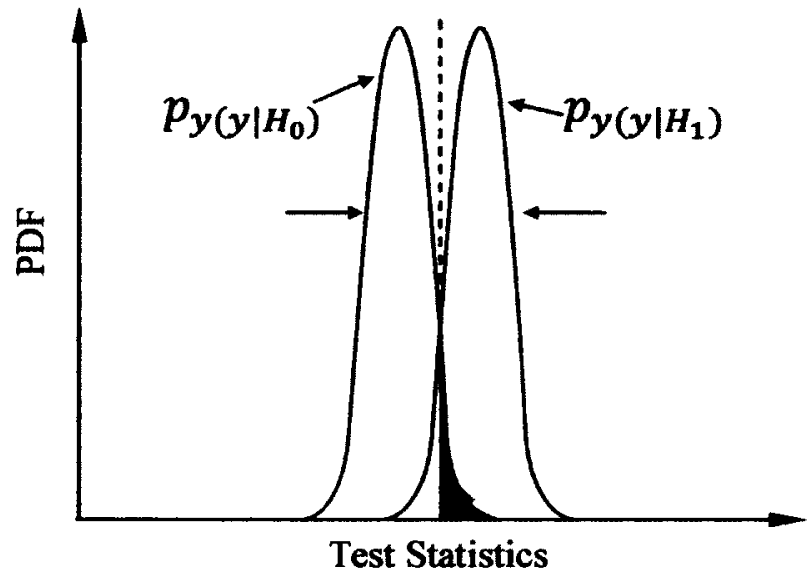

(b) Reduce Noise Power

Figure 3.4. Sensing Performance SNR vs. Noise Power $P_{F A}$ and $P_{D}$ Trade-Of

In spectrum sensing and signal detection, another function that arises in performance analysis of partially coherent, differentially coherent, and noncoherent communications is defined as the Marcum Q-function:

$$
Q_{M}(\alpha, \gamma)=\int_{\gamma}^{+\infty} t \cdot e^{-\left[\frac{t^{2}+\alpha^{2}}{2}\right]} \cdot I_{0}(\alpha t) d t
$$

where $\alpha$ is the maximum allowable false alarm probability, $I_{0}(\alpha t)$ is the modified Bessel function of the first kind. The Marcum Q-function is used for example as a cumulative distribution function (CDF) for noncentral chi-squared and Rice distributions. 


\subsection{Primary Transmitter Detection}

Three techniques have shown promise for OFDM transmitter detection: "matched filter", "energy detection" and "feature detection" (Figure 3.5).

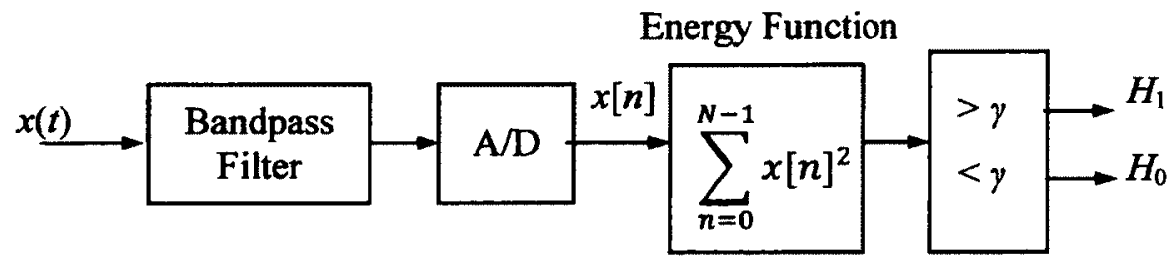

(a) Energy Detector

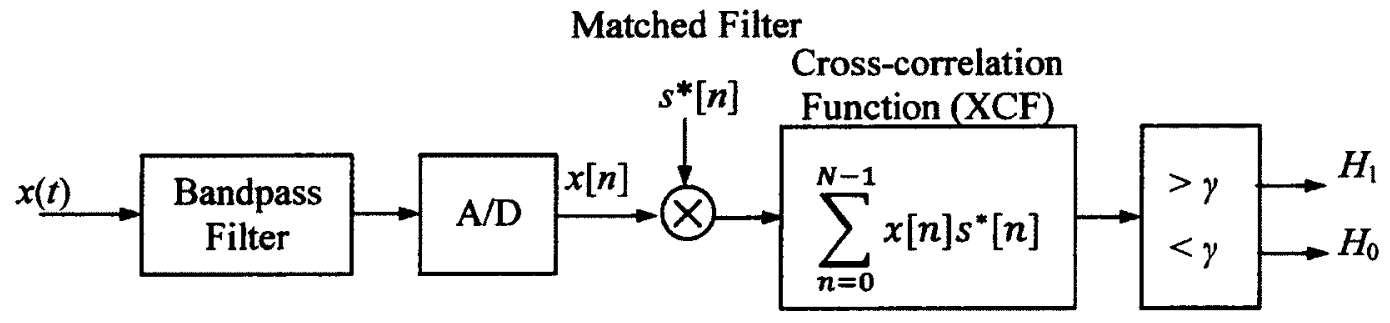

(b) Matched Filter Detector

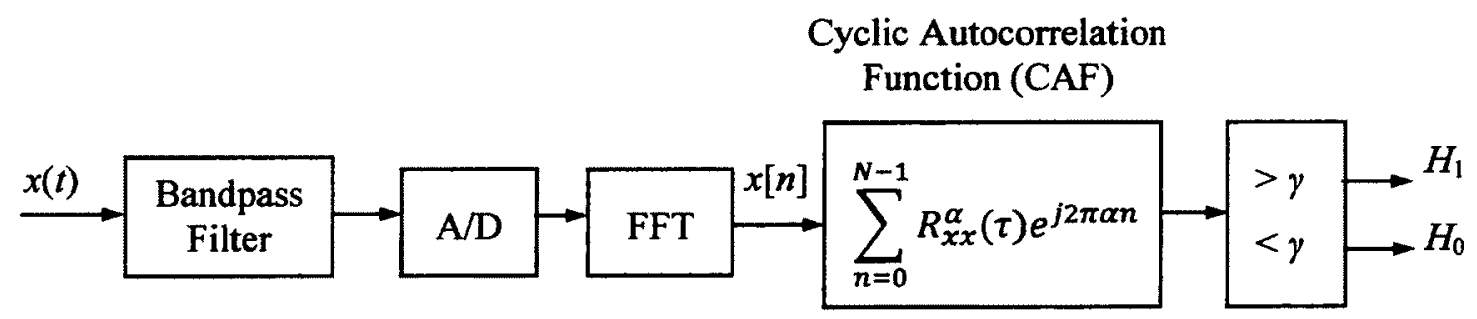

(c) Cyclostationary Feature Detector

Figure 3.5. Basic Primary Transmitter Detection Methods

\subsubsection{Energy Detection}

In energy detection [3], the signal is detected by comparing the output of the energy detector with a threshold which depends on the noise floor (Figure 3.5(a)). The main drawback is its inability to differentiate interference from primary users and noise, and its poor performance under low SNR values.

The energy detector consists of a square law device followed by a finite time integrator. The output of the integrator at any time is the energy of the input to the squaring device over the interval $T$ in the past. The noise pre-filter serves to limit the noise bandwidth; the noise at the input to the squaring device has a 
band-limited, flat spectral density. Generally, packet detection can be described as a binary hypothesis test:

$$
y(t)= \begin{cases}w(t) & H_{0}: \text { Signal absent } \\ x(t) \cdot h+w(t) & H_{1}: \text { Signal present }\end{cases}
$$

where $x(t)$ is the transmitted signal, $h$ is the wireless channel gain, and $w(t)$ is the additive white Gaussian noise (AWGN). The actual test is usually of the form that tests whether a decision variable $m_{\mathrm{n}}$ exceeds a predefined threshold $\gamma$. The packet detection case is shown below:

$$
\begin{array}{ll}
H_{0}: m_{\mathrm{n}}<\gamma & \text { Signal absent } \\
H_{1}: m_{\mathrm{n}} \geq \gamma & \text { Signal present }
\end{array}
$$

The simplest algorithm for finding the start edge of the incoming signal is to measure the received signal energy. When a desired signal is incoming, its received signal strength depends on the power setting of the transmitter and on the total path loss from the transmitter to the receiver.

$$
m_{n}=\sum_{k=0}^{L-1} r_{n-k} r_{n-k}^{*}=\sum_{k=0}^{L-1}\left|r_{n-k}\right|^{2}
$$

When there is no signal being received, the received signal $r_{\mathrm{n}}=w_{\mathrm{n}}$. When a signal starts, the received energy is increased by the signal component $r_{\mathrm{n}}=s_{\mathrm{n}}+w_{\mathrm{n}}$. The decision variable $m_{\mathrm{n}}$. This type of sum is called a sliding window.

$$
m_{\mathrm{n}+1}=m_{\mathrm{n}}+\left|r_{\mathrm{n}+1}\right|^{2}-\left|r_{\mathrm{n}-\mathrm{L}+1}\right|^{2}
$$

The difficulty of sliding window detection is how to determine the threshold $(\gamma)$.

\subsubsection{Matched Filter Detection}

A matched filter [4] is a filter used in communications to "match" a particular transit waveform as shown in Figure 3.5(b). It passes all the signal frequency components while suppressing any frequency components where there is only noise, and allows the maximum amount of signal power to pass. The purpose of the matched filter is to maximize the signal to noise ratio at the sampling point of a bit stream 
and to minimize the probability of undetected errors received from a signal. Matched filter detection will be discussed in Chapter 4 as one of the data-aided (DA) synchronization techniques using pilot.

\begin{tabular}{|l|c|c|c|}
\hline \multicolumn{1}{|c|}{ Signal Type } & NTSC & ATSC & Wireless Mic \\
\hline Sensitivity (dBm) & -94 & -116 & -107 \\
\hline SNR (dB) & 1 & -21 & -12 \\
\hline
\end{tabular}

Table 3.1. Spectrum Sensing Receiver Sensitivity Requirements for CR

Table 3.1 shows an example of the receiver sensitivity and signal-to-noise ratio for spectrum sensing of TVWS (TV white space) and wireless microphone requirements for IEEE 802.22 [13].

\subsubsection{Cyclostationarity Feature Detection}

The main advantage of the cyclostationarity detection [5] is that it discriminates the noise energy from modulated signal energy as shown in Figure 3.5(c). This is due to the fact that wideband noise has no spectral correlation while modulated signals are cyclostationary with spectral correlation due to the embedded redundancy of the signal periodicities. Cyclic-feature detection techniques have the ability to perform a signal-timing measurement, discriminate against signals not of interest using sufficiently long collects, and reduce its sensitivity to unknown and changing background noise levels and interference activity. In addition, information such as the carrier frequency and chip rate can be calculated according to the cyclic frequencies. Another reason why procedures based on cyclostationarity are attractive in the area of signal detection is that they are robust against random noise and interference and thus have particularly good performance at the low SNR regime. However, computational complexity of feature detection methods is high in comparison with energy detection methods. Cycle detection methods do require that the signal of interest exhibit cyclostationarity, and the single-cycle detectors also require knowledge of the value of a cycle frequency. Thus, modifications of the modulation schemes that destroy, substantially weaken, or vary the cyclostationarity of the signal are needed to prevent interception by cycle detection. Cyclostationary detectors also require longer observation times than energy detectors. Therefore, spectral "holes" that are short in time cannot be exploited as efficiently as with sensing methods that require less sensing time. However, as was highlighted in [FCC 2003a], feature detectors 
can achieve a huge processing gain over radiometric detectors. A feature detector can be capable of receiving signals more than $30 \mathrm{~dB}$ below the noise floor. The hidden node problem that might result in missing the presence of a signal becomes much less likely than with radiometric detectors.

A cyclostationary detector is a non-coherent detector due to the quadratic transformation. However, it coherently detects features and thus has a processing gain with respect to energy detector. In cyclostationary detection, we can utilize one of three main Cyclic Autocorrelation Function (CAF) functions: the continuous cyclic autocorrelation, the discrete cyclic autocorrelation function, and the spectral correlation function (SCF) or spectral density function (SDF). These are expressed below:

The continuous cyclic autocorrelation is defined as:

$$
R_{x x^{*}}^{\alpha}(\tau)=\frac{1}{T} \int_{-\frac{T}{2}}^{\frac{T}{2}} R_{x x^{*}}\left(t+\frac{\tau}{2}, t-\frac{\tau}{2}\right) e^{-j 2 \pi \alpha} d t
$$

where $R_{x x^{*}}(t)$ is the linear autocorrelation function.

The discrete cyclic autocorrelation function is given by:

$$
R_{x x^{*}}^{\alpha}(k)=\frac{1}{T} \sum_{n=0}^{N-1} x\left(n+\frac{k}{2}\right) x^{*}\left(n-\frac{k}{2}\right) e^{-j 2 \pi f \alpha n}
$$

where

$$
a=\frac{1}{T}(n=0,1,2, \ldots) \text { is cycle frequency, which is important in cyclostationary detection. }
$$

$T$ is the cycle.

The spectral correlation function (SCF) also known as spectral density function (SDF) is expressed as:

$$
S_{x x^{*}}^{\alpha}(f)=\int_{-\infty}^{\infty} R_{x x *}^{\alpha}(\tau) e^{-j 2 \pi f \tau} d \tau
$$

Blind carrier frequency synchronization approaches, which rely on the signal statistics, often have very high computational complexity. 
The advantages of the matched filter detection method include: optimal signal detection and demodulation performance, low computational cost, and maximized signal to noise ratio. The disadvantages of the matched filter detection method are: a requirement of a prior knowledge of the primary user (Coherent), and a requirement for a special receiver for each primary user

The advantages of the energy detection method are: simple (sub-optimal) detection, no requirement for any prior info (Non-coherent), a simple algorithm and low computational cost, an optimum detector when the signal is completely unknown, and no specific HW requirements. The disadvantages of the energy detection method include: poor performance under low signal-to-noise ratio (SNR) values, an inability to operate in low SNR, no ability to distinguish users sharing the same channel, an inability to differentiate interference from primary users and noise, and lower performance. The advantages of the cyclostationary detection method include: robustness in low SNR, robustness to interference, remarkable performance, and the ability to differentiate noise from primary users' signals. The disadvantages of the cyclostationary detection method include: computationally complexity, high implementation cost, a requirement for partial information of primary user, long observation times, signal specific response, and poorer performance as compared to energy detector based sensing methods when the noise is stationary.

\subsubsection{Waveform-Based/Autocorrelation-Based Sensing}

In waveform-based sensing, known patterns are usually utilized in wireless systems to assist synchronization. Such patterns include preambles, midambles, regularly transmitted, spreading sequences etc. Waveform-based sensing requires short measurement time. Waveform-based sensing refers to the detection of known transmitted patterns contain in the received signal, and increases the accuracy of the detector with a relatively low complexity. The waveform-based sensing requires short measurement time and is more robust than the energy detector or cyclostationarity based methods. Waveform-based sensing is also known as Correlation detector and will be discussed in details in Chapter 4. These methods are known as the non data-aided (NDA) synchronization techniques using cyclic prefix (CP). 


\subsubsection{Radio Identification-Based Detection}

In the radio identification approach, several features such as transmission frequency, transmission range, modulation technique, etc. are extracted from the received signal, which are used for selecting the most suitable secondary user technology for the CR transmission. In this approach, CR users decide the transmission parameters and technology suitable according to the sensed information.

\subsubsection{Multi-Resolution Spectrum Sensing (MRSS)}

Hur et al. [26] have proposed a wavelet approach for spectrum sensing by combing coarse and fine sensing resulting in a Multi Resolution Spectrum Sensing (MRSS) approach as shown in Figure 2.23 [26]. The basic idea is by correlating the received signal with the modulated wavelet, the spectral contents of the received signal around the carrier frequency in the given band are obtained. It is suggested that the coefficients of the Wavelet Transform are generated by correlating the received signal with a given wavelet basis function in the analog domain. The wavelet basis function is changed to calculate another wavelet transform coefficient. These wavelet transform coefficients are then used to decide if a channel is occupied. The coefficients are converted to digital format using a low-speed ADC.

\subsubsection{Covariance-Based Detection}

Zeng and Liang [27] have proposed covariance-based signal detection whose main idea is to exploit the covariance of the signal and noise since the statistical covariance of the signal and noise are usually different. These covariance properties of the signal and noise are used to differentiate the signal from the noise where the sample covariance matrix of the received signal is computed based on the receiving filter.

\subsubsection{Multi-Taper Spectrum Sensing and Estimation (MTSE)}

Thomson has proposed Multi Taper Spectrum Estimation [20], in which the last $N$ samples of a received signal are collected in a vector form and are represented as a set of Slepian base vectors. The Slepianbased vectors are used to identify the spectrum opportunities in the targeted spectrum band. The main 
idea of this approach is to utilize its fundamental property, that is, the Fourier transforms of Slepian vectors have the maximal energy concentration in the bandwidth $f_{c}-\mathrm{W}$ to $f_{\mathrm{c}}+\mathrm{W}$ under a finite samplesize constraint. After MTSE, by analyzing this feature, CR users can identify whether there is spectrum opportunity or not. This method is also regarded as an efficient method for small sample spaces.

\subsubsection{Filter Bank-Based Spectrum Sensing (FBSE)}

Filter Bank Based Spectrum Sensing [28] is a simplified version of the MTSE by introducing only one prototype filter for each band. The main idea of FBSE is to assume that the filters at the receiver and transmitter sides are a pair of matched root-Nyquist filters $H(z)$. Specifically, the FBSE has been proposed for multi-carrier modulation based CR systems by using a pair of matched-root-Nyquist-filters. As noted, FBSE is a simplified version of the MTSE, it uses the same concept of maximal energy concentration in the bandwidth $f_{\mathrm{c}}-\mathrm{W}$ to $f_{\mathrm{c}}+\mathrm{W}$. Based on this information, spectrum occupancy can be obtained to identify the spectrum opportunities. For comparison, MTSE is better for small sample spaces whereas FBSE is better for large number of samples. Furthermore, the MTSE approach increases the computational complexity and hence might not be suitable for a CR system in which the CR users have to sense the wideband regime.

\subsection{Cooperative Detection}

Cooperative detection (data fusion) refers to spectrum sensing methods where information from multiple CR users are incorporated for primary user detection. Cooperative sensing decreases the probabilities of misdetection and false alarm considerably. It can solve hidden primary user problem and it can decrease sensing time. Cooperative detection uses a control channel to share the spectrum sensing results known as fusion center. Collaborative spectrum sensing is most effective when collaborating CRs observe independent fading or shadowing channels. The cooperative detection provides more reliable detection by incorporated information from multiple CR users. However, this approach requires additional overhead traffic and encompasses certain level of complication. 


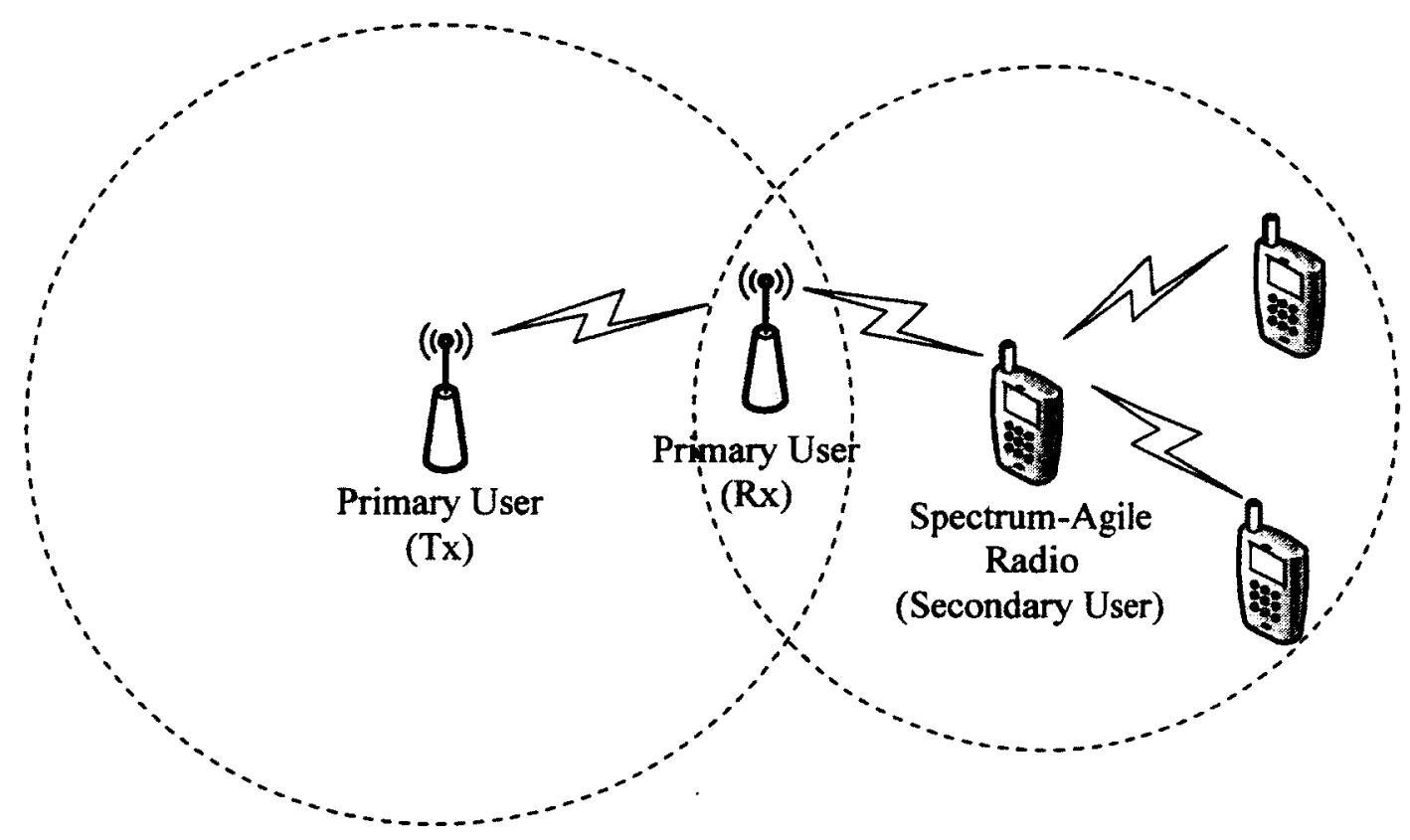

Figure 3.6. Hidden Primary User Problem in Spectrum-Agile Radio

When spectrum sensing is performed using a single sensor [32], that sensor may be in a deep fade, e.g., it may be shadowed, relative to a transmitter one wishes to detect. This is known as the hidden node problem. Because of this possibility a secondary unit basing its decisions on single sensor sensing may not engage in a secondary transmission unless it is highly confident in its detection of a spectrum opportunity (i.e., it must be able to detect a transmitter even as it experiences deep fading). To this end, the sensing node must use conservative detection thresholds and/or highly sensitive receivers, which cause high false alarm probability (the probability of reaching a "detect" decision when there was nothing there) and/or high cost devices, respectively.

Figure 3.6 shows the hidden primary user problem caused by many factors including severe multipath fading or shadowing observed by secondary users while scanning for primary users' transmissions. The dashed circles show the operating ranges of the primary user and spectrum-agile radio device. Here, spectrum-agile radio device causes unwanted interference to the primary user (receiver) as the primary transmitter's signal could not be detected because of the locations of devices. Cooperative sensing is proposed in the literature for handling the hidden primary user problem. 


\subsubsection{Centralized Sensing}

In centralized sensing, a central unit collects sensing information from cognitive devices, identifies the available spectrum, and broadcasts this information to other cognitive radios or directly controls the cognitive radio traffic. Only the cognitive radios with reliable information are allowed to report their decisions to the central unit.

\subsubsection{Distributed Sensing}

In the case of distributed sensing, cognitive nodes share information among each other but they make their own decisions as to which part of the spectrum they can use. Only final decisions are shared in order to minimize the network overhead due to collaboration.

\subsubsection{External Sensing}

An external agent performs the sensing and broadcasts the channel occupancy information to the cognitive radios.

\subsection{Interference-Based Detection}

The interference-based detection is proposed to address the Primary User (PU) uncertainty problem by working the decision based on the cumulative RF energy from multiple transmissions measured at the receiver. The spectrum band is considered available if the aggregate RF energy does not exceed the set maximum interference limit. Signal sensing and adaptive technology, such as that now used for ATPC (Automatic Temperature Power Control), will become increasing sophisticated and could play a major role in the self-regulation of interference. Such technology could be used in conjunction with the interference temperature metric to ensure that the condition of the RF environment does not exceed permissible levels. 


\subsubsection{Primary Receiver Detection}

The spectrum opportunities are detected based on the PU receiver's local oscillator leakage power.

\subsubsection{Interference Temperature Management}

In this approach, the CR system works as an UWB technology where the secondary users coexist with primary users and are allowed to transmit with low power and restricted by the interference temperature level so as not to cause harmful interference to primary users. Spectrum underlay (Interference-Tolerant) imposes a constraint on the transmission power, where the secondary users access the network by spreading their signals over a wide frequency band. The underlay approach imposes severe constraints on the transmission power of secondary users. Operating below the noise floor of primary users, the secondary users are allowed to interfere with primary users up to a certain tolerable level. Spectrum overlay (Interference-Free) imposes constraints on when and where a secondary user (SU) can transmit, and occurs where secondary users access the portion of the spectrum that is not used by primary users. As a result, there is virtually no interference to the primary users (PUs).

Figure 3.7 shows several primary transmitter detection methods. The matched filter has the highest accuracy and complexity since we need many filters for spectrum sensing over a wideband regime. The energy detector is the least accurate and least complex, since we do not need any special kind of fitters.

Figure 3.8 shows the horizontal and vertical spectrum sharing. Horizontal sharing occurs where CR users and primary users have equal opportunities to access the spectrum such as in wireless LAN operating at $2.4 \mathrm{GHz}$, and in order to improve the overall system performance, CR users can choose the channels which have less traffics or less number of users. Thus, CR users and primary users coexist in the system and use the bands simultaneously. Vertical sharing occurs where CR users have less preference over the primary users. Moreover, in vertical sharing, CR systems need operator's assistant. 


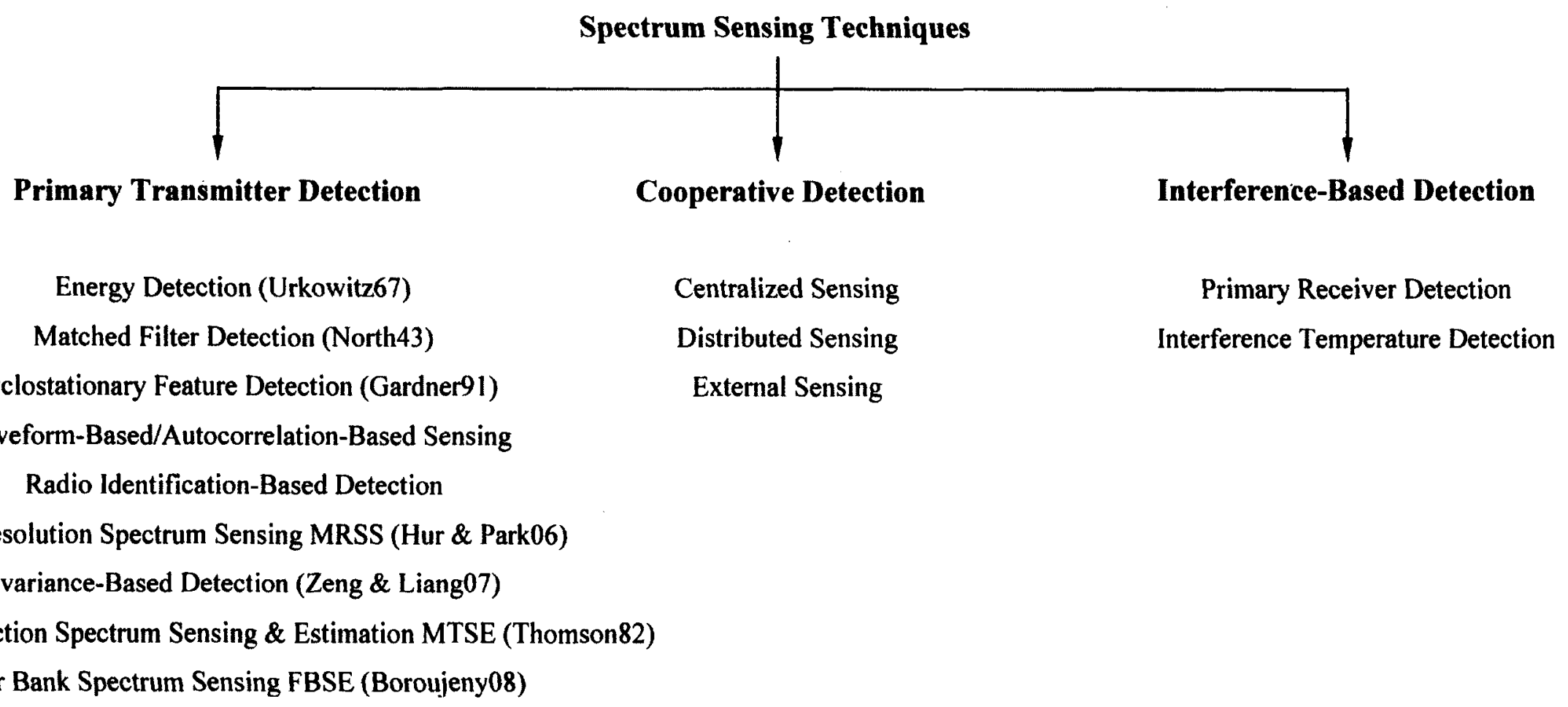

Figure 3.7. Detecting Spectrum Opportunities: Spectrum Sensing Techniques

Figure 3.7 show the classification of the major spectrum sensing methods: primary transmitter detection, cooperative detection and interferencebased detection. 
Figure 3.8 shows the complexity and accuracy trade-off between different spectrum sensing methods. While energy detection method is the simplest but least accurate detection method, whereas matched filter detection method is the most accurate but also requires the highest level of design complexity.

Figure 3.9 shows the horizontal and the vertical spectrum sharing. Depending on the regulatory status of the incumbent radio systems, cognitive radios share spectrum with different types of systems.

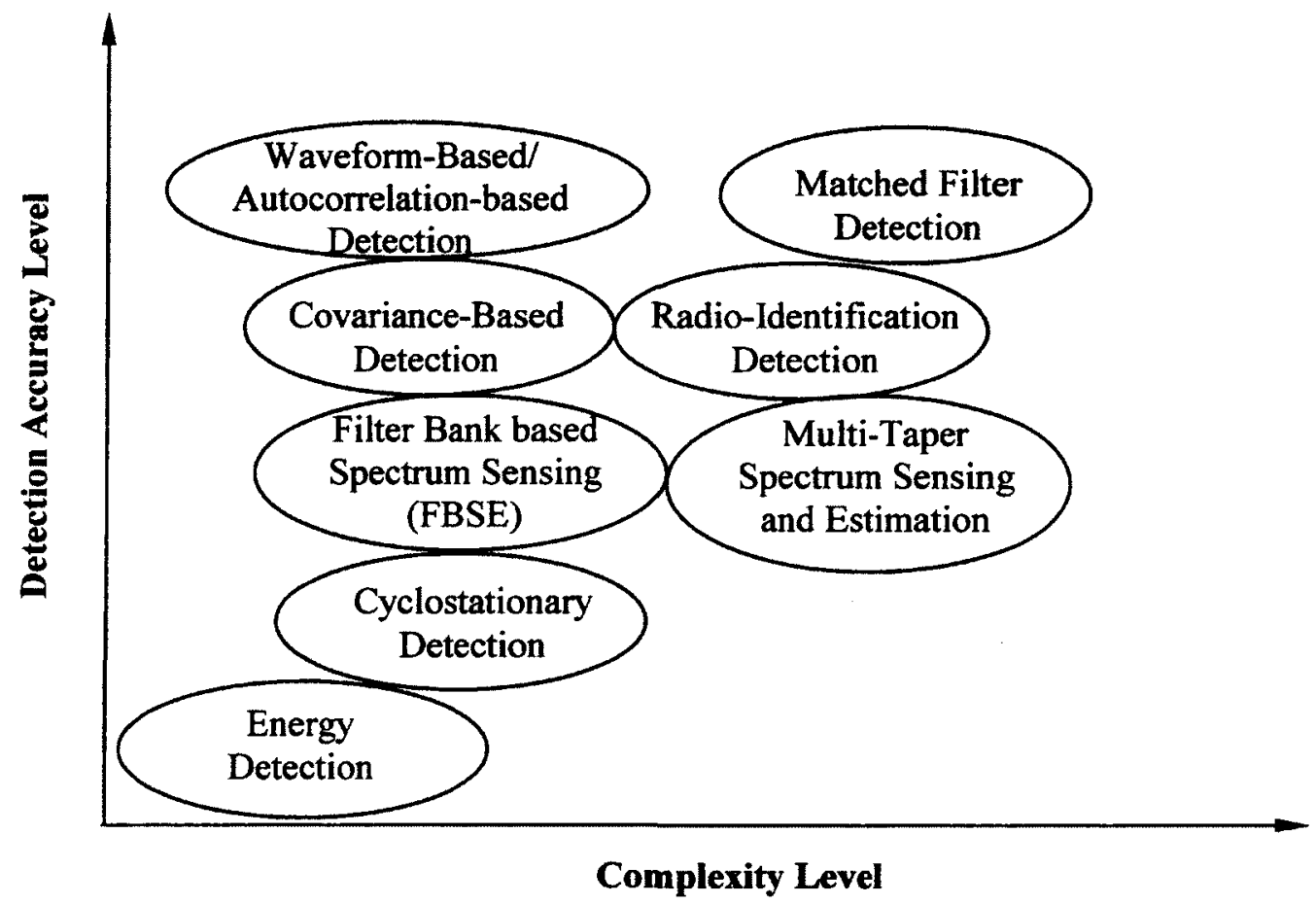

Figure 3.8. Primary User (PU) Detection Complexity vs Accuracy Tradeoff

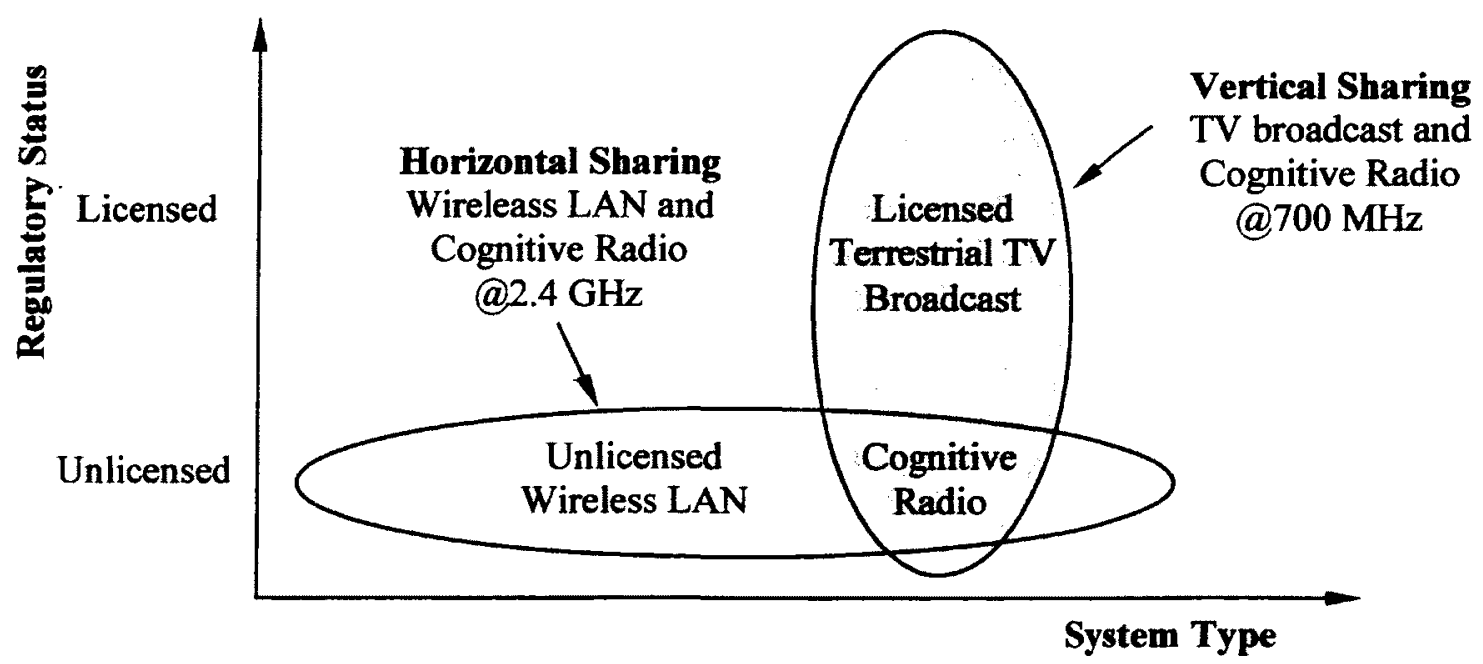

Figure 3.9. Licensed and Unlicensed Spectrum Sharing 


\subsection{Conclusion}

This chapter has presented an overview of spectrum sensing techniques. In summary, several major spectrum sensing techniques have been classified and presented. Spectrum sensing is an important enabling technology for future opportunistic spectrum access. One major category of spectrum sensing is transmitter detection. There exist several different approaches for transmitter detection which may be used in different sensing scenarios. The most well-known methods are perhaps energy detection, cyclostationary detection and matched filter detection, and these have been presented and discussed in this chapter.

The simplest method to detect the presence of a signal is by energy detection (no a priori information needed). An energy detector measures the energy in a radio resource and compares the value against a threshold. If and only if the measured energy is below the threshold, the radio resource is declared as not occupied, just what is required for opportunistic use. Energy detection can be used for declaring whether a resource is occupied or not, but it cannot be used to identify the type of system or user (e.g., primary or secondary) that is occupying the channel. Also, an energy detector needs to have an idea of the noise level to adjust the detection threshold.

In some applications, some parts of the signal one wishes to detect can be known a-priori; for instance, the synchronization words for GSM, preambles for WiMAX, and synchronization signals (P-SCH, S$\mathrm{SCH}$ ) for LTE. In this case we can utilize a matched filter detector. The match filter detector can be shown to be optimal in the sense that it maximizes the SNR of a received single-path signal in AWGN. A matched filter detector works by correlating the received signal with the pattern one wishes to detect. Thus, the amplitude and the phase of the signal are extracted. If this magnitude is above a threshold value, a detection decision is made. Generally, matched filter detection has very good detection capabilities. However, it requires a priori information which may not be available for all applications. 
Cyclostationary detection is typically a statistical test based on the estimated autocorrelation function of one or several known cyclic frequencies. A cyclostationary process has statistical properties which vary periodically over time. A widesense cyclostationary process (the analogue of a wide sense stationary process) has an autocorrelation function which is cyclic with a certain periodicity. Communication signals are typically cyclostationary with multiple periodicities, e.g., the cyclic prefix. However, cyclostationary detection will only be able to detect a limited amount of systems for which the communication signals possess known cyclostationary properties. By the same token, these systems can be explicitly identified by the cyclostationary detector.

An approach to solve the hidden primary user problem, but requires some coordination, is cooperative sensing, in which multiple sensors are utilized [32]. If the sensor measurements are independent and identically distributed the probability that a collaborative sensing detects other spectrum usage becomes $P_{c d}=1-\left(1-P_{d}\right)^{N}$, provided a "one out of $N$ " detection approach is used, where $P_{d}$ is the probability of detection for a single sensor (the probability of obtaining a "detect" decision when the system was indeed present) and $N$ is the number of sensors. This can be a significantly increased detection probability compared to $P_{d}$ for a single sensor, and thus one can be more aggressive in the detection by exchanging a reduced $P_{c d}$ for a decreased probability of false alarm $P_{f a}$.

Cooperative sensing takes advantage of geographical varieties of secondary sensing nodes which experience different channel conditions. Spatially distributed sensing nodes measure the signal from the primary user using opportunistic spectrum access (OSA), and report the sensed measurement results to the fusion center. The fusion center makes the final decision about the primary user spectrum availability based on the collected measurement results. 


\section{Chapter 4. Synchronization Techniques}

\subsection{Introduction}

In this chapter, several estimation methods and the latest research on synchronization techniques are investigated for frequency-agile radios from the LTE and OFDM perspective. We will discuss some synchronization techniques that will eliminate the ISI and ICI effects. That is, if the length of the guard interval (CP) is set longer than or equal to the maximum delay of a multipath channel, the ISI effect of an OFDM symbol on the next symbol is confined to be within the guard interval so that it may not affect the FFT of the next OFDM symbol. This implies that a guard interval longer than the maximum delay of the multipath channel allows for the maintenance of the orthogonality among the subcarriers.

In OFDM, ISI can be eliminated by inserting a cyclic prefix with length greater than the channel impulse response, and ICI can be avoided by maintaining the orthogonality of carriers under the condition that the transmitter and the receiver have the exact same carrier frequency. However, in the real world, frequency offsets will arise from the frequency mismatch of the transmitter and the receiver oscillators and the existence of Doppler shift in the channel.

Generally, OFDM receiver synchronization is performed in two steps: acquisition and tracking. Acquisition is a process to obtain an initial rough or coarse estimate of timing and frequency parameters. Tracking is an on-going process where this rough estimate is refined to get a better estimation. Acquisition parameter estimation schemes generally have wide range, but low accuracy. Tracking has a narrower range and finer accuracy. The goal in symbol timing offset (STO) estimation is to find the correct timing of a frame, a place to start the $N$-point FFT for demodulating an OFDM symbol. The goal in carrier frequency offset (CFO) estimation is to maintain or preserve the orthogonality properties of the sub-carriers, since a frequency error results in inter-carrier interference. 
Synchronization algorithms for OFDM systems can be divided in two main categories: data-aided (DA) and non-data-aided or blind (NDA).

\subsection{Data-Aided (DA) and Non Data-Aided (NDA) Methods}

The data-aided (DA) category uses a training sequence or pilot symbols for estimation (Figure 4.1). It has high accuracy and low calculation, but reduces the usable bandwidth or reduces the data transmission speed. The synchronization time needs to be as short as possible, and the accuracy must be as high as possible for high packet rate transmission. However, the use of pilot symbols inevitably decreases the capacity and/or throughput of the overall system, thus making them suitable only in a startup/training mode. Also, the SNR at the front of the receiver is often too low to effectively detect pilot symbols, thus a blind approach is usually much more desirable.

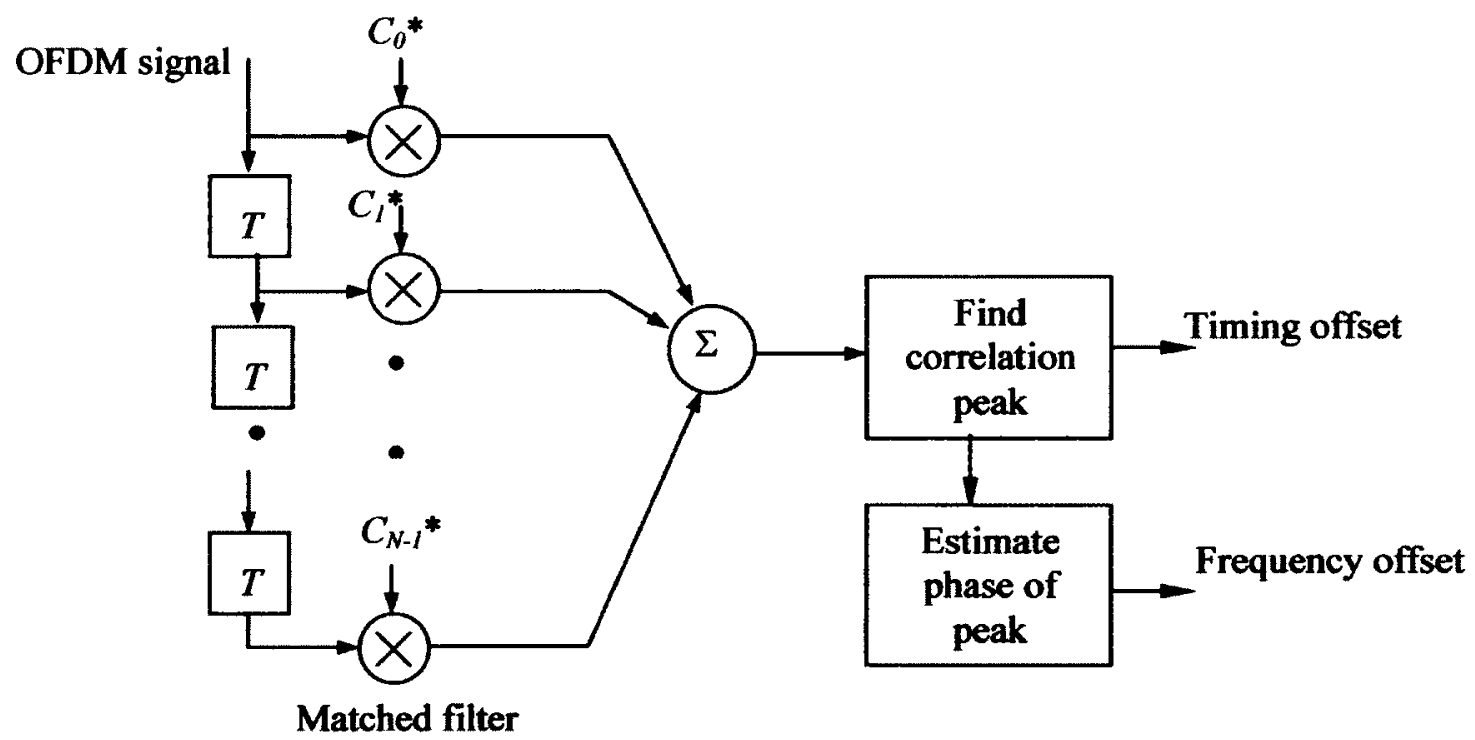

DA: Use Preamble (Matched filter), PN Code, Training Symbol, or Pilot

Figure 4.1. Data-Aided Sync Method using Training Sequence ( Matched Filter)

The non-data aided (NDA) category often uses the cyclic prefix correlation (Figure 4.2). It doesn't waste bandwidth and reduce the transmission speed, but its estimation range is too small, to be suitable for acquisition. 


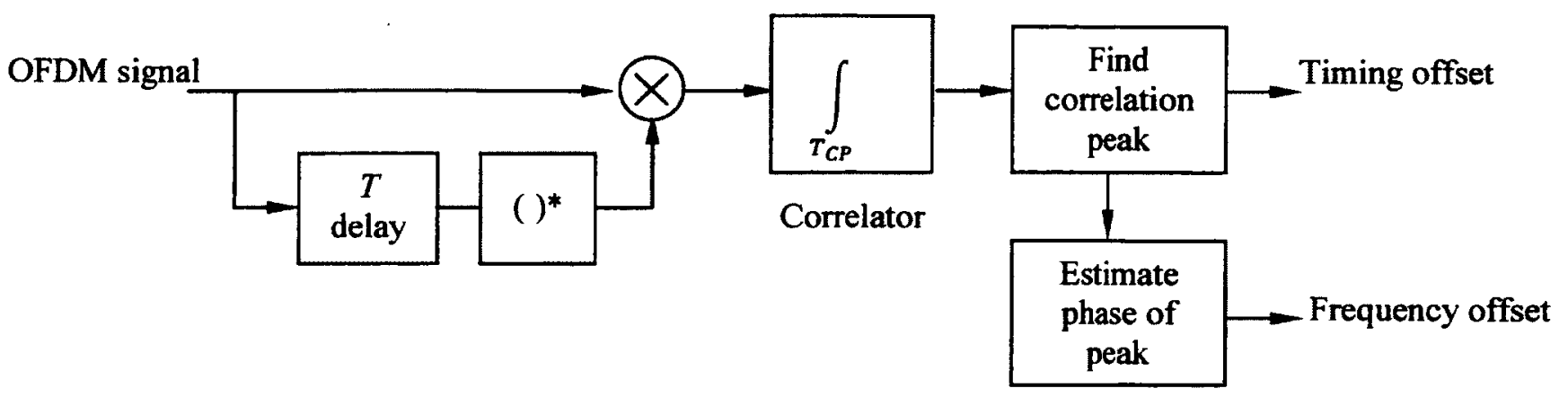

NDA: Use Cyclic Prefix or signal statistics (blind).

Figure 4.2. Non-Data-Aided Synchronization Method using Cyclic Prefix (Correlator)

An OFDM transmission symbol $x(n)$ is given by the $N$-point complex modulation sequence :

$$
x(n)=\frac{1}{N} \sum_{k=0}^{N-1} X(k) \cdot e^{\frac{j 2 \pi n k}{N}}
$$

After passing through the channel, the received sequence $y(n)$ can be expressed as:

$$
y(n)=\frac{1}{N} \sum_{k=0}^{N-1} X(k) \cdot H(k) \cdot e^{\frac{-j 2 \pi n(k+\varepsilon)}{N}}+w(n)
$$

where $\varepsilon$ represents the carrier frequency offset, and $n$ is the time index.

The demodulated signal $Y(k)$ for the $k^{\text {th }}$ subcarrier (i.e., at the FFT output) consisting of three components:

$$
Y(k)=\sum_{n=0}^{N-1} y(n) \cdot e^{-\frac{j 2 \pi k n}{N}}
$$

where $k$ represents the frequency index.

$$
\begin{gathered}
Y(k)=\sum_{n=0}^{N-1} \frac{1}{N} X(k) \cdot H(k) e^{\frac{j 2 \pi n \varepsilon}{N}}+\sum_{\substack{l=0 \\
l \neq k}}^{N-1} \frac{1}{N} X(l) \cdot H(l) e^{\frac{j 2 \pi n(l-k+\varepsilon)}{N}}+\sum_{n=0}^{N-1} W(n) \cdot e^{-\frac{j 2 \pi k}{N}} \\
Y(k)=X(k)+I(k)+W(k)
\end{gathered}
$$

where $\widehat{X}(k)$ represents the desired signal, $I(k)$ is the ICI, and $W(k)$ is the AWGN. 
- The first term $X(k)$ is the estimate of $X(k)$ which experiences an amplitude reduction and phase shift due to the carrier frequency offset $\varepsilon$.

$$
\widehat{X}(k)=X(k) \cdot H(k) \frac{\sin (\pi \varepsilon)}{N \sin (\pi \varepsilon / N)} e^{j \pi \varepsilon(N-1) / N}
$$

- The second term $I(\mathrm{k})$ represents the inter-carrier interference caused by the frequency offset:

$$
I(k)=\sum_{\substack{l=0 \\ l \neq k}}^{N-1} X(l) \cdot H(l) \frac{\sin (\pi(l-k+\varepsilon))}{N \sin (\pi(l-k+\varepsilon) / N)} e^{\frac{j \pi(\varepsilon(N-1)-l+k)}{N}}
$$

- The third term $W(k)$ is the added white Gaussian noise (AWGN).

Assuming that all subcarriers is shifted with the same carrier frequency offset.

\subsection{Sliding Window Detection Method}

The sliding window detection algorithm calculates two consecutive sliding windows of the received energy. The basic principle is to form the decision variable $m_{\mathrm{n}}$ as a ratio of the total energy contained inside the two windows. Figure 4.3 (a) shows the case when only the noise is received. The response $m_{\mathrm{n}}$ is flat. Figure 4.3 (b) shows the case when the packet edge starts to cover the A window. The energy in the A window increases until the point where $A$ is totally contained inside the start of the packet. After this point the B window starts to also collect signal energy, and when it is also completely inside the received packet, the response of $m_{n}$ is flat again.

$$
a_{n}=\sum_{m=0}^{M-1} r_{n-m} r_{n-m}^{*}=\sum_{m=0}^{M-1}\left|r_{n-m}\right|^{2} ; \quad b_{n}=\sum_{l=0}^{L} r_{n+l} r_{n+l}^{*}=\sum_{l=0}^{L}\left|r_{n+l}\right|^{2} ; \quad m_{n}=\frac{a_{n}}{b_{n}}
$$



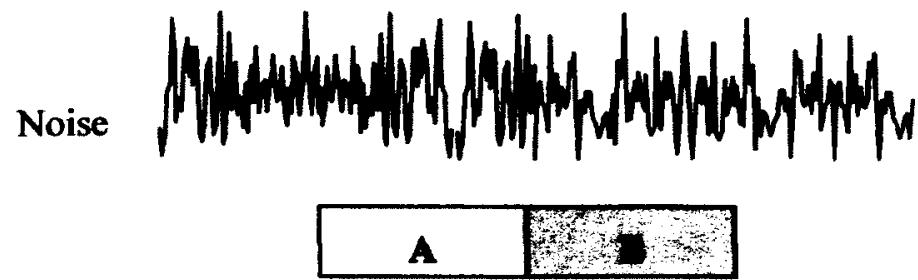

Threshold

$m_{\mathrm{n}}$

(a)

Packet
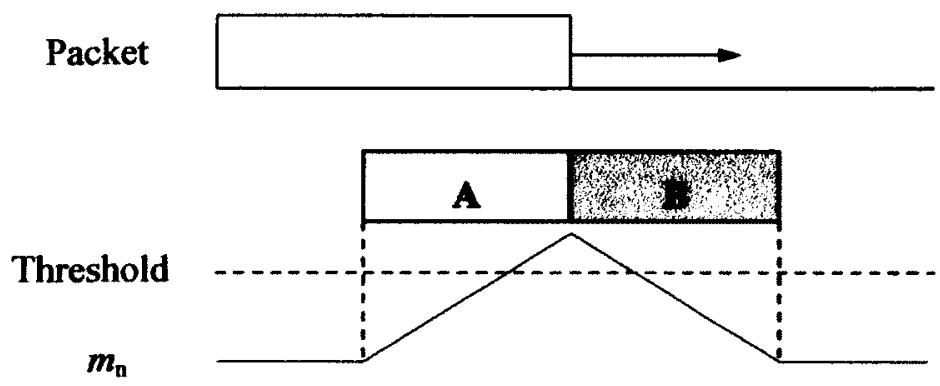

(b)

Figure 4.3. Sliding Window Detection Algorithm

\subsection{Symbol Time Offet (STO) Estimation Techniques}

Symbol synchronization refers to the task of finding the precise moment an individual OFDM symbol starts and end. The symbol timing result defines the FFT window, i.e. the set of samples used to calculate FFT of each received OFDM symbol.

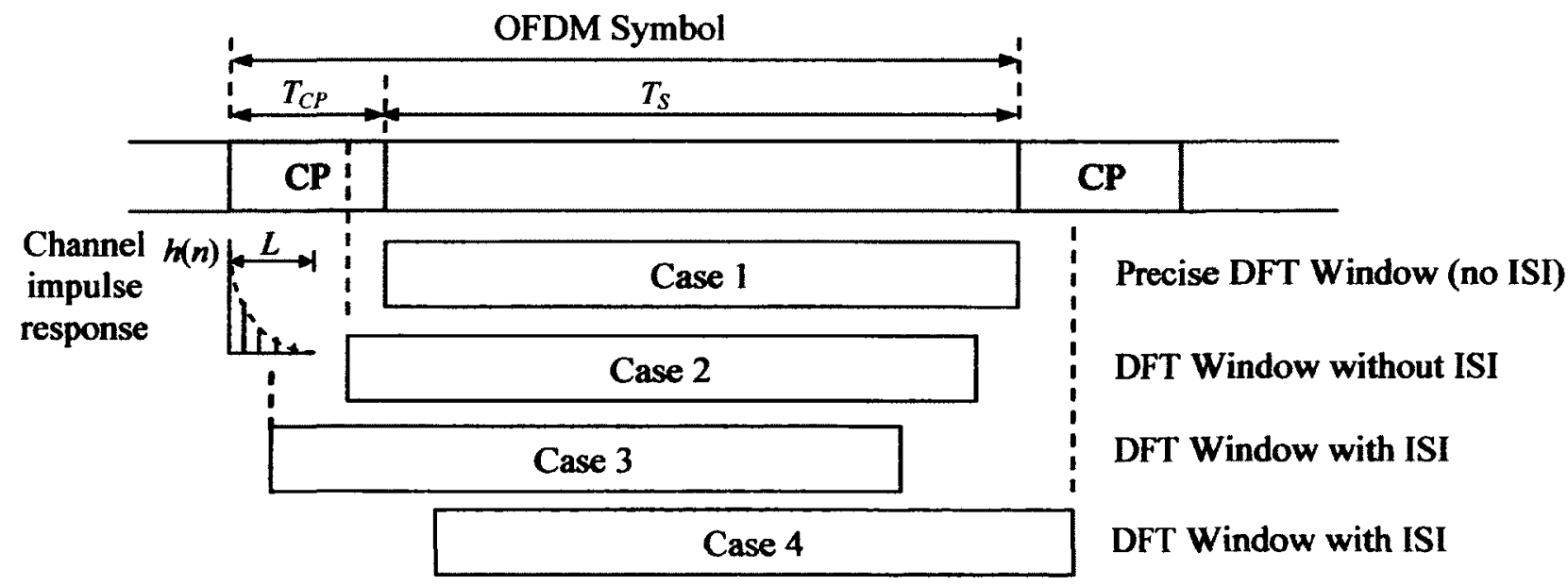

Figure 4.4. Scenarios of DFT Window Location Symbol Offset Synchronization 
Four distinct cases has been identified as shown in Figure 4.4 .

Case 1: This is the case when the estimated starting point of an OFDM symbol coincides with the exact timing, preserving the orthogonality among subcarrier frequency components. In this case, the OFDM symbol can be perfectly recovered without any type of interference.

Case 2: This is the case when the estimated starting point of anOFDM symbol is before the exact point, yet after the end of the (lagged) channel response to the previous OFDM symbol. In this case, the $l^{\text {th }}$ symbol does not overlap with the previous $(l-1)^{\text {th }}$ OFDM symbol. As a result, no ISI with the previous symbol occurs in this case.

Case 3: This is the case when the starting point of the OFDM symbol is estimated prior to the end of the the previous OFDM symbol, and thus, the symbol timing is too early to avoid ISI. In this case, the orthogonality among subcarrier components is destroyed by the ISI (from the previous symbol) and furthermore, ICI (Inter-Channel Interference) occurs.

Case 4: This is the case when the estimated starting point of the OFDM symbol is after the exact point, which means the symbol timing is a little later than the exact one. As a result, the orthogonality has been destroyed.

Cyclic prefix is a portion of an OFDM symbol used to absorb inter-symbol interference (ISI) caused by any transmission channel time dispersion. The use of cyclic prefix converts a serial frequency-selective fading wideband channel into a set of parallel flat-fading narrowband independent channels.

It is noted that, frame-based OFDM signals (both burst and continuous modes) are applicable to both time-domain and frequency-domain synchronization, whereas packet-based OFDM signals (burst mode only) are applicable only to time-domain synchronization. 


\subsubsection{Non Data-Aided (NDA) Timing Offset Estimation}

The maximum likelihood (ML) estimator developed by Van de Beek et al. [7] exploits the redundancy in the cyclic prefix and cross-correlation between samples inside the cyclic prefix and samples $\mathrm{N}$ points away. These cyclic prefix-based schemes are generally used for fine-frequency synchronization only. Although this estimator was originally derived for an AWGN channel, it can also be applied to a dispersive channel. However, for a dispersive channel, there will be additional noise in the estimator due to the ICI and ISI. Figure 4.5 shows the synchronization method block diagram using a cyclic prefix.

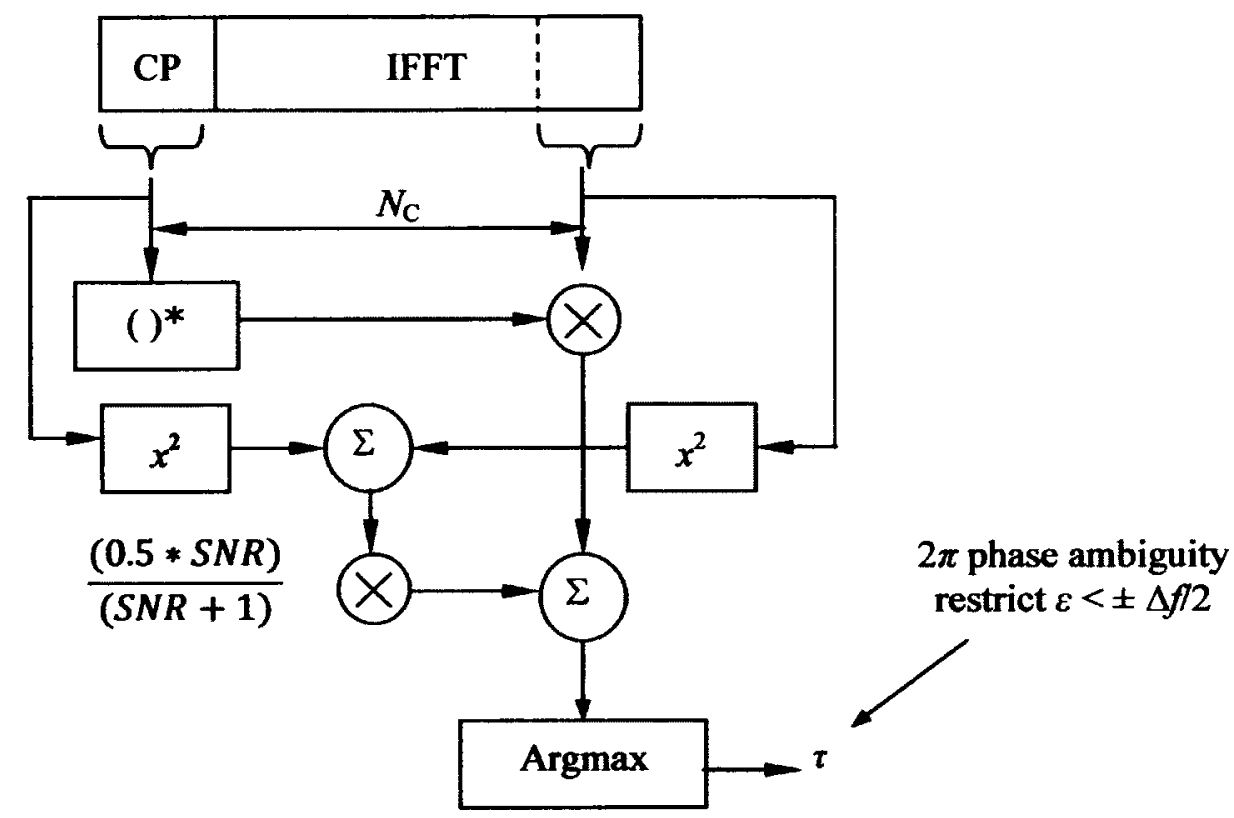

Figure 4.5. Block Diagram of Synchronizer using Cyclic Prefix

Non-data-aided methods exploit the statistical redundancy of the received signal. The transmitted signal is modelled following a Gaussian process. The basic idea behind these methods is that the cyclic prefix of the transmitted signal yields information about where an OFDM symbol is likely to start. In addition, the transmitted signal's redundancy also contains useful information about the carrier frequency offset. 


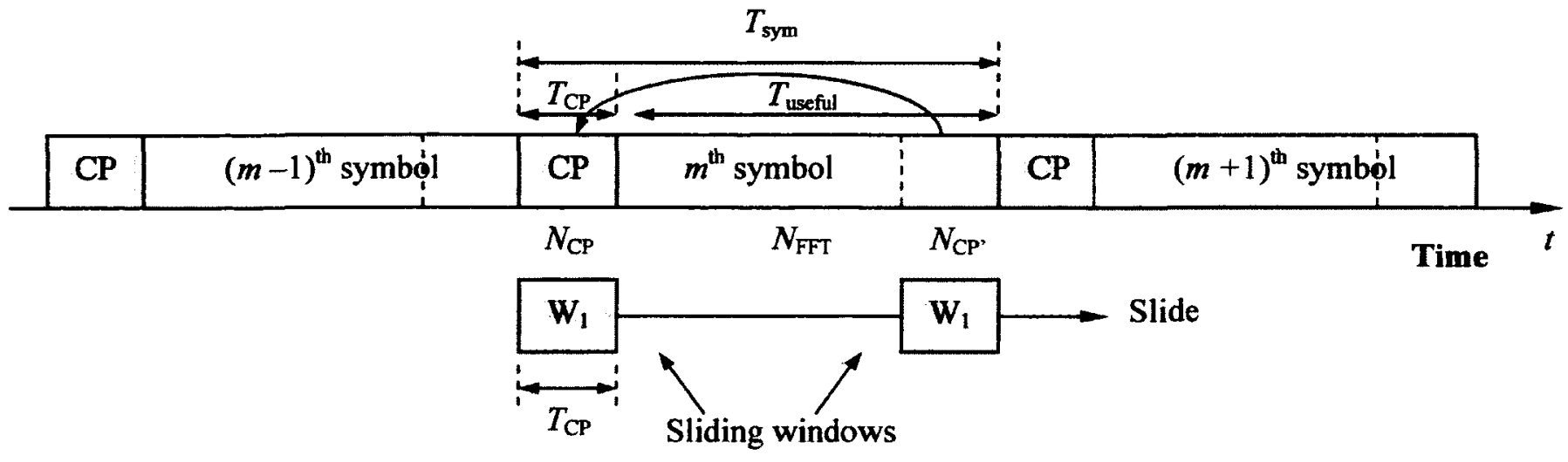

Figure 4.6. Non Data-Aided (NDA) STO Estimation Technique using Cyclic Prefix

The cyclic prefix is the prepended replica of the data portion in the OFDM symbol as shown in Figure 4.6. Consequently, the $\mathrm{CP}$ and the corresponding data part share their similarities that can be used for the symbol timing offset (STO) estimation. As shown in Figure 4-6 above, $N_{\mathrm{CP}}$ samples and another $N_{\mathrm{CP}^{\prime}}$ samples are spaced $N_{\text {FFT }}$ samples apart. Now, consider two sliding windows, $W_{1}$ and $W_{2}$ which are spaced $N_{\text {FFT }}$ samples apart. These windows can slide to find the similarity between the samples within $W_{1}$ and $W_{2}$. The similarity between two blocks of $N_{\mathrm{CP}}$ and $N_{\mathrm{CP}}$ samples in $W_{1}$ and $W_{2}$ is maximized when the cyclic prefix of an OFDM symbol falls into the first sliding window $W_{1}$. This is shown in Figure 4.7. This maximum point can be used to identify the symbol time offset (STO). The use of a cyclic prefix in the transmitted signal has the disadvantage of requiring more transmit energy and the frequency offset estimation range is limited to half of the subcarrier spacing.

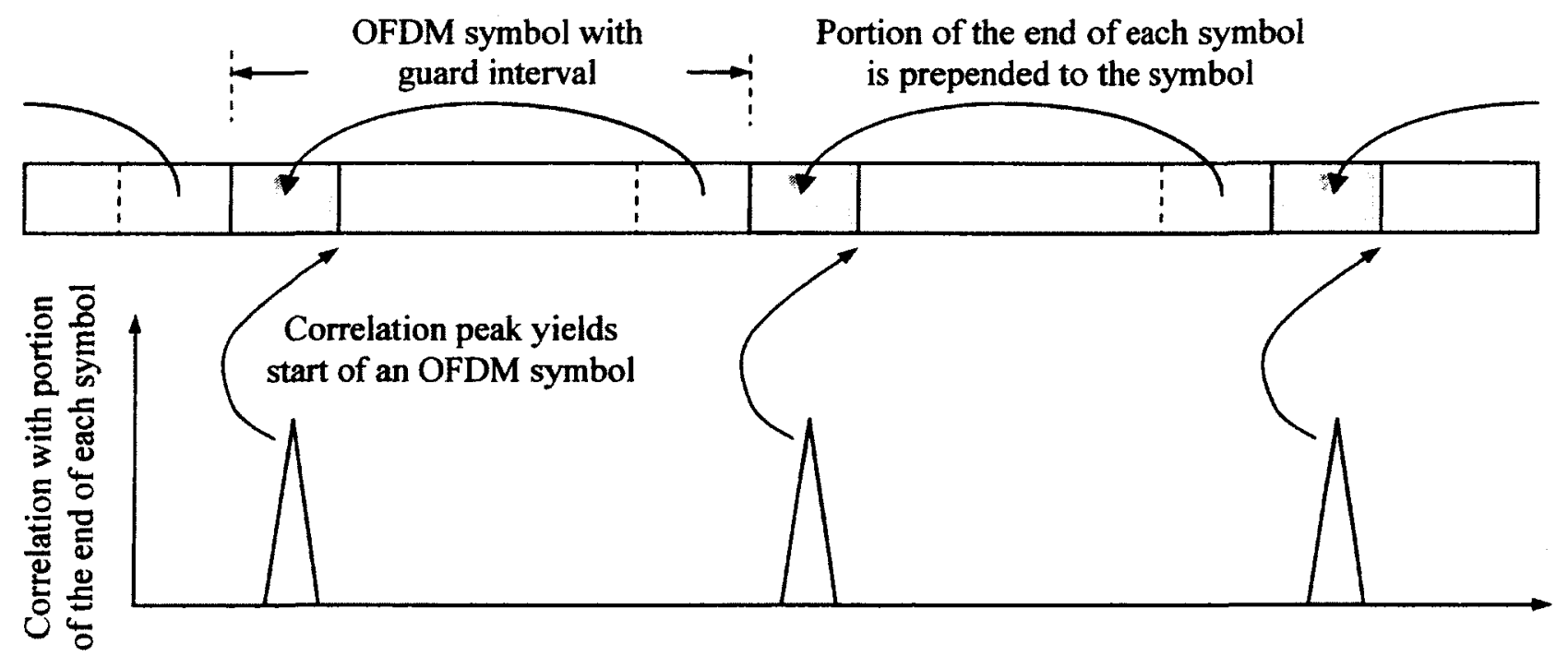

Figure 4.7. Time Synchronization based on Cyclic Prefix Correlation 
Therefore, the cyclic prefix of an OFDM signal can be utilized in a timing/frequency acquisition algorithm. The premise is that distinct peaks being one symbol interval apart will show up in the correlation result if the correct parameters are chosen.

The coarse symbol timing synchronization is first executed in the time-domain and then, the fine symbol timing is performed in the frequency-domain to ensure a more accurate estimation.

Although timing offset estimation methods based on the redundancy of the cyclic prefix are derived for an AWGN channel, with minor modification, these methods can also be used for dispersive channels. If the length of the channel is less than the size of the cyclic prefix, there will be a window at the end of the cyclic prefix without any ISI from the previous symbol.

\subsubsection{Data-Aided (DA) Timing Ofiset Estimation}

The most well-known auto-correlation method is probably the so-called Schmidl \& Cox97 method [6]. This method considers a preamble consisting of two OFDM symbols placed at the beginning of the frame as shown in Figure 4.8. The first symbol has identical halves in the time-domain, so that the correlation between these two halves can be carried out in the receiver to find the timing metric. However, the metric suffers from a plateau as shown in see Figure 4-8, which leads to some uncertainty in the determination the start of the frame.

This can be used for coarse frequency acquisition. In this case, there are two identical symbols with pilots. The first one is used for timing and fine-frequency acquisition. The second one is combined with the first one for coarse frequency acquisition. 


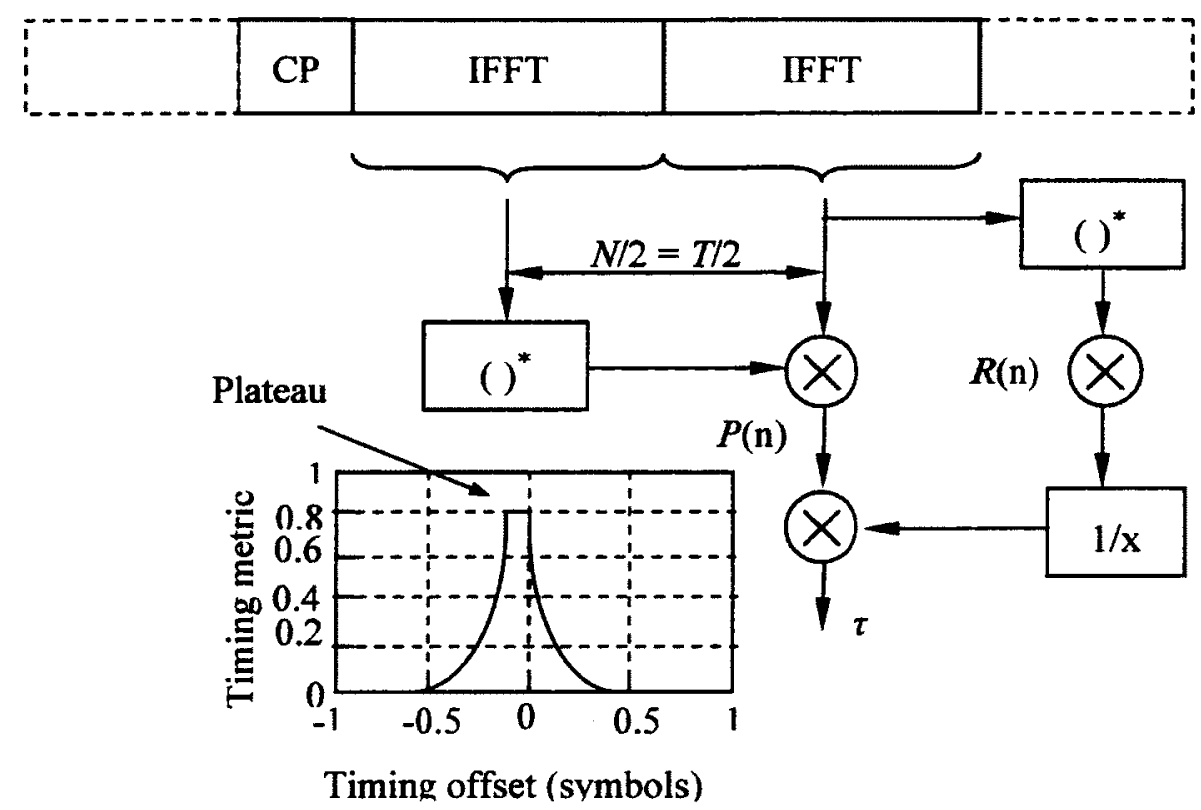

Figure 4.8. Timing Synchronization using Training Sequence

Figure 4.9 shows a block diagram of the symbol timing synchronization method using training sequences [6]. Training sequence repetition is a widely used technique to characterize propagation channels and gives good results about phase rotation due to any frequency error. In principle, fine timing synchronization is done after removing the frequency error.

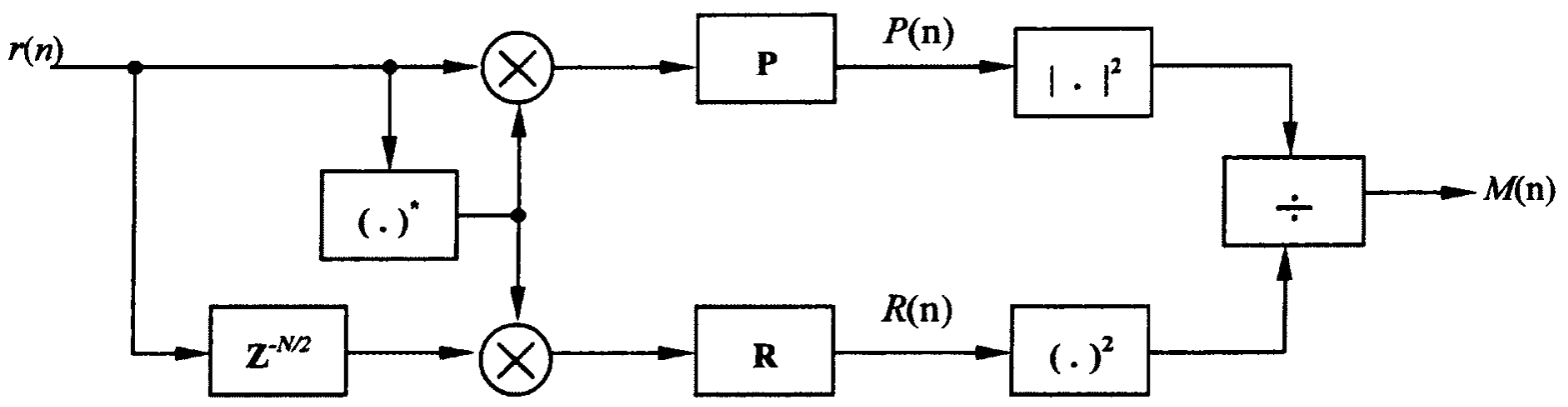

Figure 4.9. Time Synchronizer using Training Symbol

In this method, the $P$ window is the autocorrelation between the received signal $r(m)$ and a delay version of the received signal $r(m+N / 2)$. 


$$
P(d)=\sum_{k=0}^{N / 2-1} r^{*}(d+k) \cdot r(d+k+N / 2)
$$

where $d$ is the time index

The $R$ window calculates the received signal energy during the auto-correlation window.

$$
R(d)=\sum_{k=0}^{N / 2-1}|r(d+k+N / 2)|^{2}
$$

The value of the $R$ window is used to normalize the decision statistic, so that it is not dependent on absolute received power level. A timing metric can be defined as:

$$
M_{S c h \text { midl }}(d)=\frac{|P(d)|^{2}}{(R(d))^{2}}
$$

The symbol timing estimate is:

$$
\hat{\tau}=\underset{d}{\arg }\{\max (M(d))\}
$$

The carrier frequency offset estimate is:

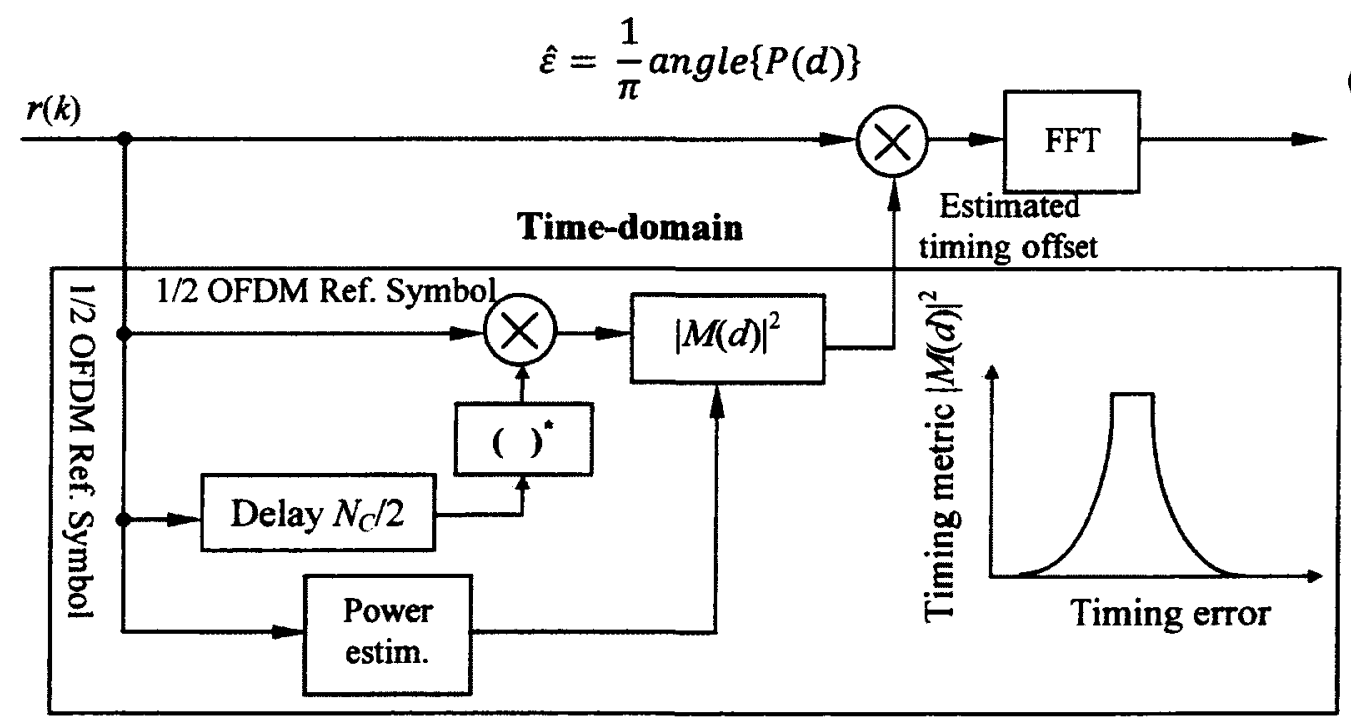

Figure 4.10. Time Synchronization using Identical Half Reference Symbol

Figure 4.10 shows Schmidl \& Cox [6] symbol timing synchronization method. 
In order to avoid the ambiguity caused by the plateau of timing metric, Minn [8] has made improvement to the Schmidl \& Cox's method and proposed two new methods. This is shown in Figure 4.11 (b). For the first method, all samples over one symbol period (excluding the cyclic prefix) are used in calculating the half symbol energy required in the timing metric, and the timing metric is averaged over a window of cyclic prefix length. For the second method, a training symbol is used containing four equal length parts but with the last two having different sign. These methods give a sharper estimation and a smaller estimator variance, but still have large MSE in an ISI channel.

A novel timing offset based on a modified version of the Minn work has been proposed by Park [9]. This method, shown in Figure 4.11 (c) uses a training symbol consisting of four parts: the first two are symmetric and the last two are the conjugate of the first two. This produces an even sharper timing metric and has significantly lower MSE than [6] and [8]. However, its timing metric has two large sidelobes which will affect the timing performance.

Shi \& Serpedin [10] have proposed a synchronization scheme improvement to [6] and [8] by exploiting the repetitive structure of a training symbol for carrier synchronization. This method achieves a superior performance with respect to [6] in terms of better detection properties and accuracy and larger frequency estimation range, which is up to two subcarrier spacing $( \pm 2 \Delta f)$. 


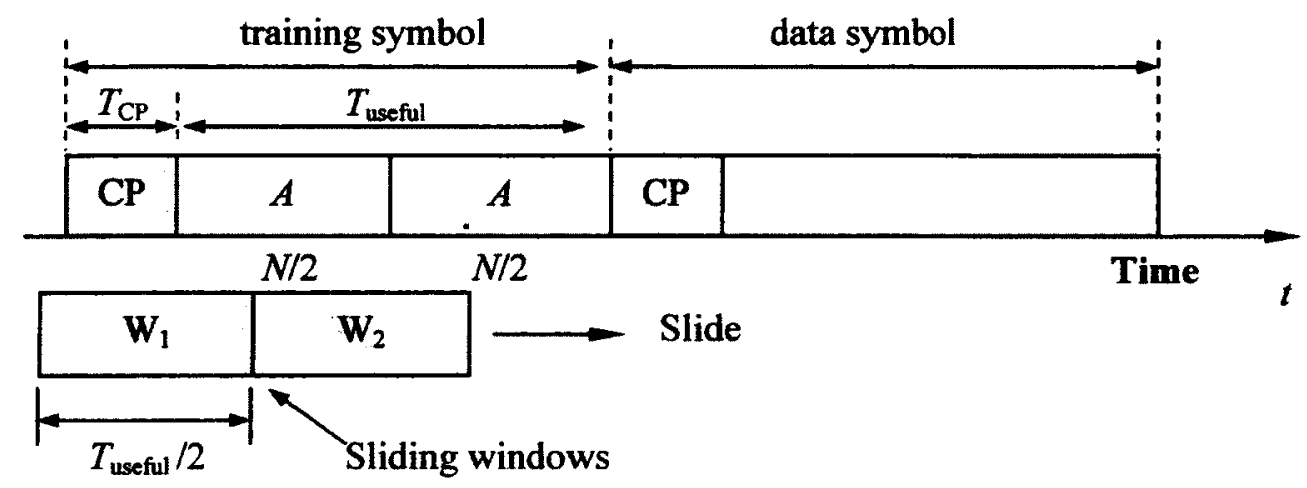

(a) Period of Training Symbol $=T_{\text {useful }} / 2[6]$

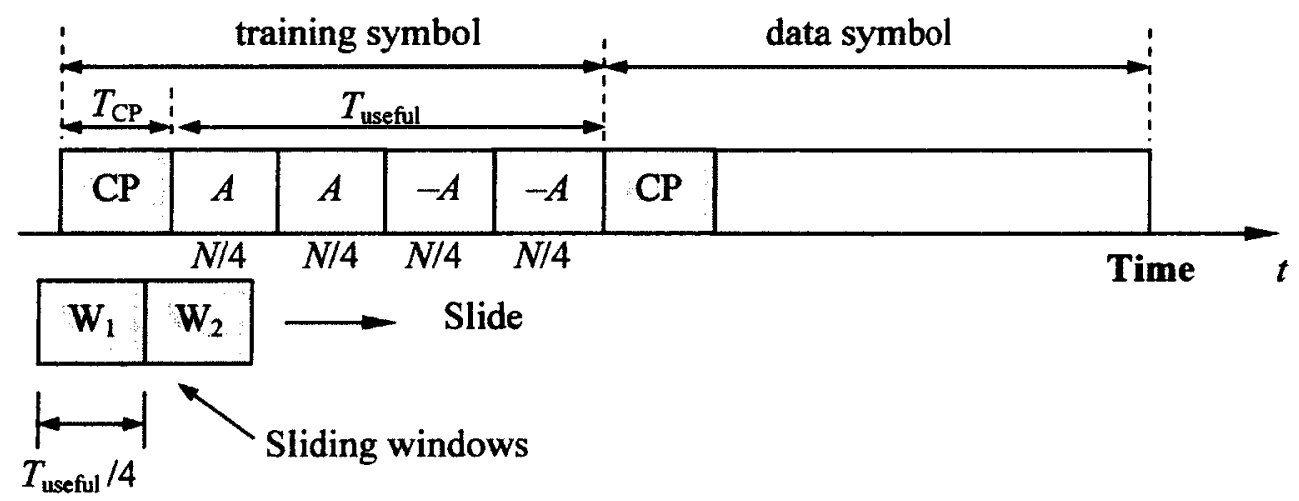

(b) Period of Training Symbol $=T_{\text {useful }} / 4[8]$

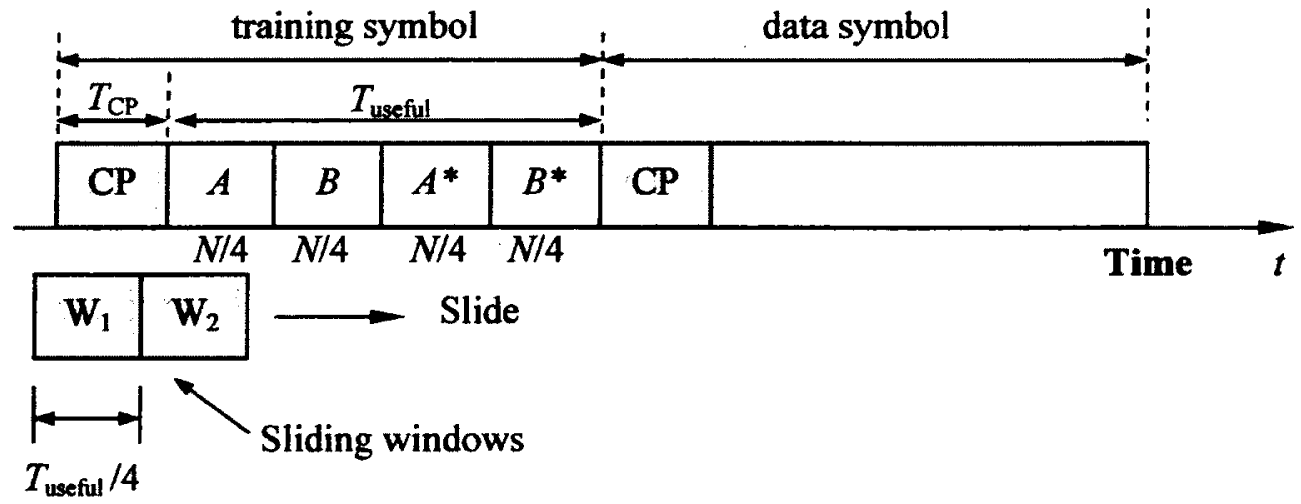

(c) Period of Training Symbol $=T_{\text {weful }} / 4$ [9]

Figure 4.11. Data-Aided (DA) STO Estimation using Training Sequence Repetition 
The timing synchronization metric of the Minn method [8] is an improvement on [6] and is given below:

$$
\begin{gathered}
P_{2}(d)=\sum_{m=0}^{1} \sum_{k=0}^{N / 4-1} r^{*}(d+(N / 2) m+k) \cdot r(d+(N / 2) m+k+N / 4) \\
R_{2}(d)=\sum_{m=0}^{1} \sum_{k=0}^{N / 4-1}|r(d+(N / 2) m+k+N / 4)|^{2} \\
M_{\text {Minn }}(d)=\frac{\left|P_{2}(d)\right|^{2}}{\left(R_{2}(d)\right)^{2}}
\end{gathered}
$$

Autocorrelation based algorithms utilize the repetition structure of the training sequence or the guard interval of OFDM symbols to acquire timing synchronization. They are usually very simple and have low implementation complexity. However, there is a well known plateau problem.

There have been some schemes employing cross-correlation for time synchronization. Although such schemes achieve fairly good accuracy, the complexity is high. There are other synchronization methods, which use the maximum-likelihood (ML) mechanism to achieve better performance. However, their computational complexity usually is much higher than the above schemes.

For fine symbol timing synchronization the mechanism relies on matching the time-domain received waveform with the preamble waveform to obtain the channel impulse response (CIR), and then the optimal symbol timing. 


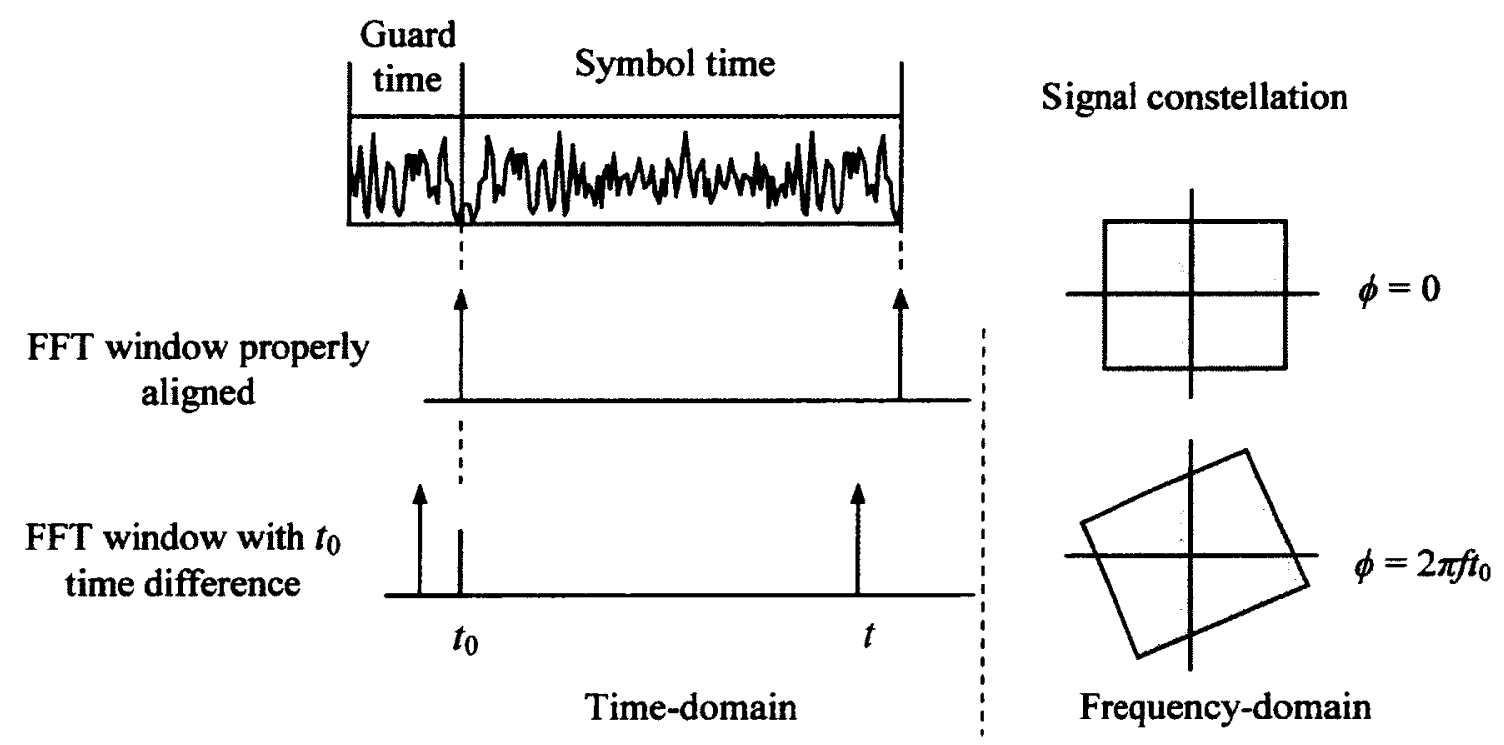

Figure 4.12. Fine Symbol Time Synchronization based on CIR Estimation

Figure 4.12 shows one method of fine symbol timing synchronization by estimation of the channel impulse response. The received signal without noise $r(t)=s(t) \otimes h(t)$ is the convolution of the transmit signal $s(t)$ and the channel impulse response $h(t)$. In the frequency-domain after FFT processing we obtain $R(f)=S(f) \cdot H(f)$. By transmitting a special reference symbol (e.g. CAZAC sequence), $S(f)$ is a priori known to the receiver. Hence, after dividing $R(f)$ by $S(f)$ and IFFT processing, the channel impulse response $h(t)$ is obtained and accurate timing information can be derived. If the FFT is not properly aligned, the received signal becomes

$$
r(t)=s\left(t-t_{0}\right) \otimes h(t)
$$

which turns into

$$
R(f)=S(f) \cdot H(f) e^{-j 2 \pi f t_{0}}
$$

after the FFT operation. After division of $R(f)$ by $S(f)$ and again performing and IFFT, the receiver obtain $h\left(t-t_{0}\right)$ and with that $t_{0}$. Finally, the fine time synchronization process consists of delaying the FFT window so that $t_{0}$ becomes quasi zero. 


\subsection{Carrier Frequency Offset (CFO) Estimation Techniques}

Many methods for CFO estimation in OFDM systems have been developed, which can be separated into two categories: data-aided methods (DA) which use the training sequences or pilot signals, and non-dataaided methods (NDA) which use the cyclic prefix methods. Carrier frequency offset estimation can be performed in two steps: coarse CFO estimation and fine CFO estimation. Coarse CFO estimation is the process of estimating the subcarrier numbering index $k_{0}$. Fine CFO estimation is the process of estimating the center frequencies of each sub-carrier $\varepsilon$. These are shown in Figure 4.13 and 4.14 respectively.

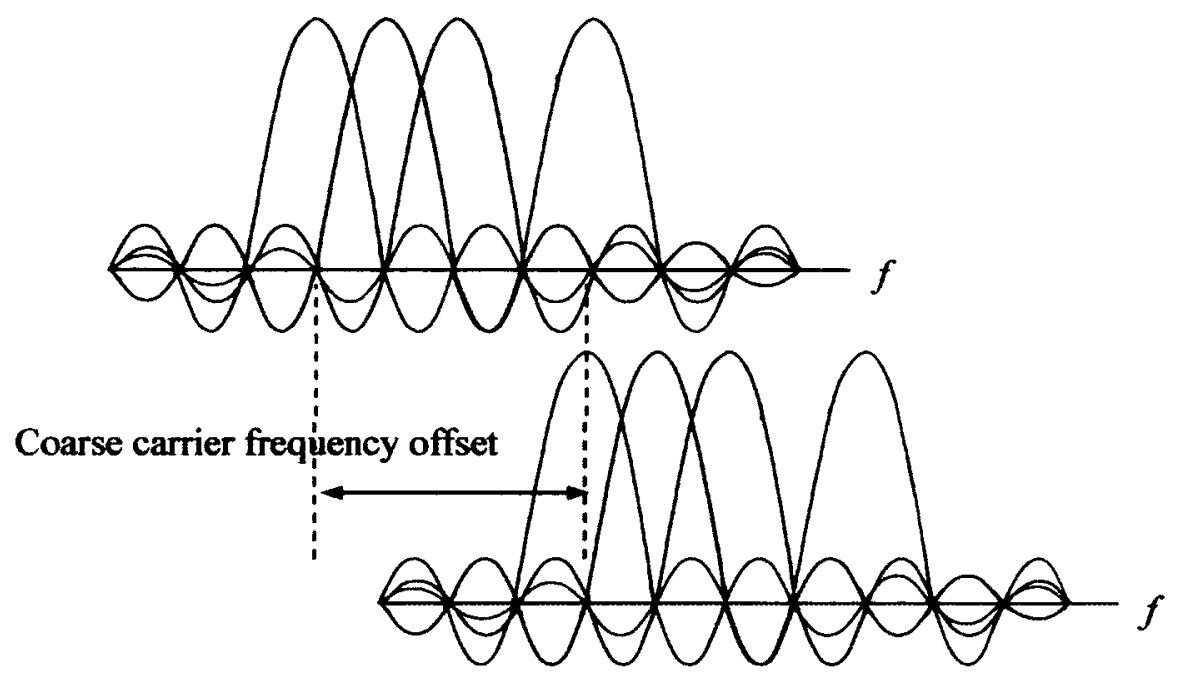

(a) Coarse CFO Synchronization

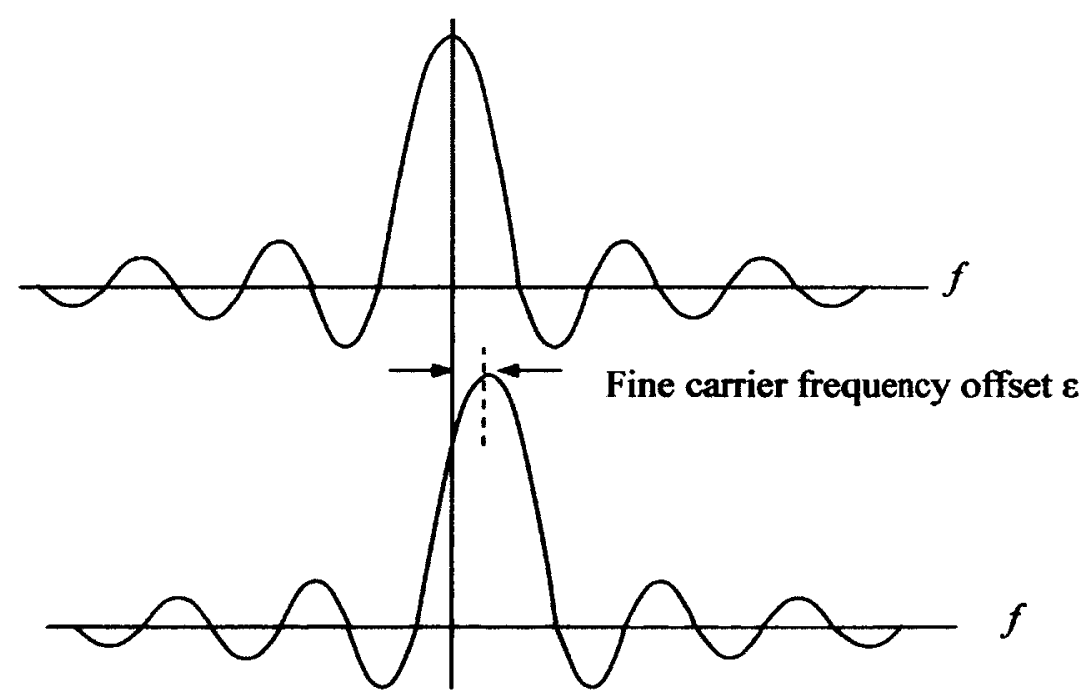

(b) Fine CFO Synchronization

Figure 4.13. Carrier Frequency Synchronization 
The integer component of the CFO represents a cyclic shift in the frequency-domain symbol, while the fractional component causes interferences between sub-channels. Fractional frequency offset is first estimated and corrected in the time domain, then the integer frequency offset is estimated and corrected in frequency domain. Frequency estimation is not a perfect process, so there is always some residual error. The main problem of the residual frequency offset is constellation rotation.

Coarse carrier frequency offset estimation is the process of estimating the subcarrier numbering index $k_{0}$. Generally, the frequency synchronization is performed in two steps in order to reduce the overall complexity. The frequency acquisition (in time-domain) requires more computational complexity and more redundancy in the transmitted signal, while the frequency tracking (in frequency-domain) employs algorithms with lower frequency capture ranges, but requires less complexity and redundancy.

In an OFDM link, the subcarriers are perfectly orthogonal only if the transmitter and receiver use exactly the same frequencies. This is impossible in practice. The frequency offset estimation is an important problem for an OFDM system. The loss of orthogonality due to carrier offset must be compensated for before discrete Fourier transform (DFT)-based demodulation can be performed.

Symbol time offset synchronization consists of: a coarse STO (CTO) estimation in the time-domain and a fine/residual STO (FTO) estimation in the frequency-domain. The frequency offset synchronization consists of: a coarse decimal/fractional CFO in the time-domain, a integral CFO in the frequency-domain, and a residual/fine CFO in the frequency-domain.

In fractional CFO, the effects are a phase shift is in the time-domain, induced the magnitude attenuation, and $\mathrm{ICl}$, and loss of orthogonality. For integer $\mathrm{CFO}$, the effects are a phase shift is in the time-domain, and there is an index shift. 


\subsubsection{Non Data-Aided (NDA) Frequency Offset Estimation}

Van de Beek et al. [7] has proposed the maximum likelihood (ML) estimator which exploits the redundancy associated to the cyclic prefix (CP) prepended in every OFDM symbol as shown in Figure 4.14 [7]. The synchronization parameters can be estimated using a single symbol. This scheme needs no training symbol, but its performance depends on the length of the CP, and its estimation range is only \pm $1 / 2$ of the subcarrier spacing. The advantage of a non data-aided methods is that no pilot symbols is required. The disadvantages are the maximum frequency offset can be corrected is usually limited to half the inter-carrier spacing, and the fact that these schemes are only effective when a large number of subcarriers are used, preferably more than 100 .

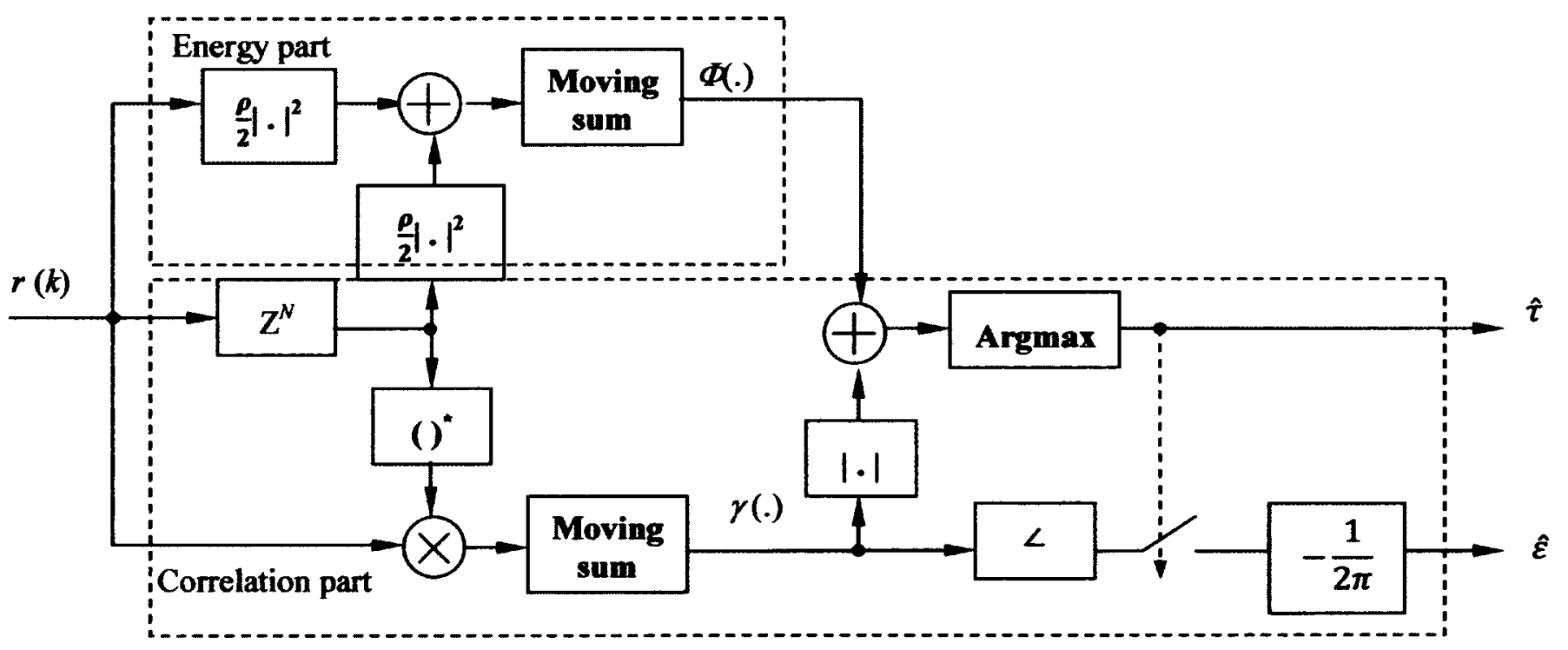

Figure 4.14. Time and Frequency Synchronizer using Cyclic Prefix

The Van de Beek ML estimator symbol timing offset is given as:

$$
\begin{gathered}
\hat{\imath}=\arg \max _{d}\left\{\left|\sum_{k=d}^{d+N_{g}-1} r(k) r^{*}(k+N)\right|-\frac{S N R}{(S N R+1)} \frac{1}{2} \sum_{k=d}^{d+N_{g}-1}|r(k)|^{2}+|r(k+N)|^{2}\right\} \\
\hat{\tau}_{M L}=\arg \max \{|\gamma(\theta)|-\rho \Phi(\theta)\}
\end{gathered}
$$


Therefore, the carrier frequency offset is:

$$
\hat{\varepsilon}_{M L}=-\frac{1}{2 \pi} \angle \gamma\left(\hat{\theta}_{M L}\right)
$$

The $\log$-likelihood function $\Lambda(\theta, \varepsilon)$ is defined (under the assumption that $r$ is a jointly Gaussian vector) as:

$$
\Lambda(\theta, \varepsilon)=|\gamma(\theta)| \cos (2 \pi \varepsilon+\angle \gamma(\theta)-\rho \Phi(\theta) \approx|\gamma(\theta)|-\rho \Phi(\theta)
$$

where $\gamma(m)$ correlates the received sampled baseband signal $r$, with a delayed version of itself:

$$
\gamma(m) \equiv \sum_{k=m}^{m+L-1} r(k) r^{*}(k+N)
$$

The function $\Phi(m)$ compensates for the different energy in the correlated samples, independent of the frequency offset $\varepsilon$.

$$
\Phi(m) \equiv \frac{1}{2} \sum_{k=m}^{m+L-1}|r(k)|^{2}+|r(k+N)|^{2}
$$

Also, $\rho$ is the correlation coefficient:

$$
\rho \equiv\left|\frac{E\left\{r(k) r^{*}(k+N)\right\}}{\sqrt{E\left\{|r(k)|^{2}\right\} E\left\{|r(k+N)|^{2}\right\}}}\right|=\frac{\sigma_{s}^{2}}{\sigma_{s}^{2}+\sigma_{n}^{2}}=\frac{S N R}{S N R+1}
$$

Now a $2 \pi$ phase ambiguity restricts the maximum value of the phase of the correlation function to $\pi$ for reliable estimation. That is, the resulting phase difference can only be determined modulo $2 \pi$ (integer phase ambiguity). The $2 \pi$ ambiguity of the phase implies that a tracking algorithm can only handle frequency errors smaller than $\Delta f / 2$. The received signal rotates faster in the time domain as CFO increases. Meanwhile, the phase differences increase linearly with time, with their slopes increasing with the CFO. If $\varepsilon>0: 5$, the phase difference exceeds $\pi$ within an OFDM symbol, which results in a phase ambiguity. This is related to the range of CFO estimation. It is noted that the angle (in radians) of $r$ is unambiguously defined only in the range $[-\pi,+\pi)$. The range (in Hertz) of the above CFO equation is $[-\pi$, $+\pi) / 2 \pi=[-0.5,+0.5)$ so that $|\varepsilon|<0.5$. Thus if the absolute value of the frequency error is larger than the acquisition range: $\hat{\varepsilon}_{\max }=[-0.5 \Delta f, 0.5 \Delta f]$, the estimation jumps to a negative value and the CFO estimate will be incorrect. 


\subsubsection{Data-Aided (DA) Frequency Offset Estimation}

Moose [29] has proposed the maximum-likelihood (ML) CFO estimation method based on two consecutive and identical training symbols. This is shown in Figure 4.15 [29]. The estimation range of the Moose's method is equal to half $\pm 1 / 2$ sub-carrier spacing. Moose increased this range by using shorter training symbols, but that reduced the estimation accuracy.

Moose carrier frequency estimate method is given as:

$$
\hat{\varepsilon}=\frac{1}{2 \pi} \tan ^{-1}\left\{\sum_{k=0}^{N-1} \operatorname{Im}\left[Y_{1}^{*}(k) Y_{2}(k)\right] / \operatorname{Re}\left[Y_{1}^{*}(k) Y_{2}(k)\right]\right\}
$$

$$
\hat{\varepsilon} \text { is the angle of } Y_{1}^{*}(k) \cdot Y_{2}(k)
$$

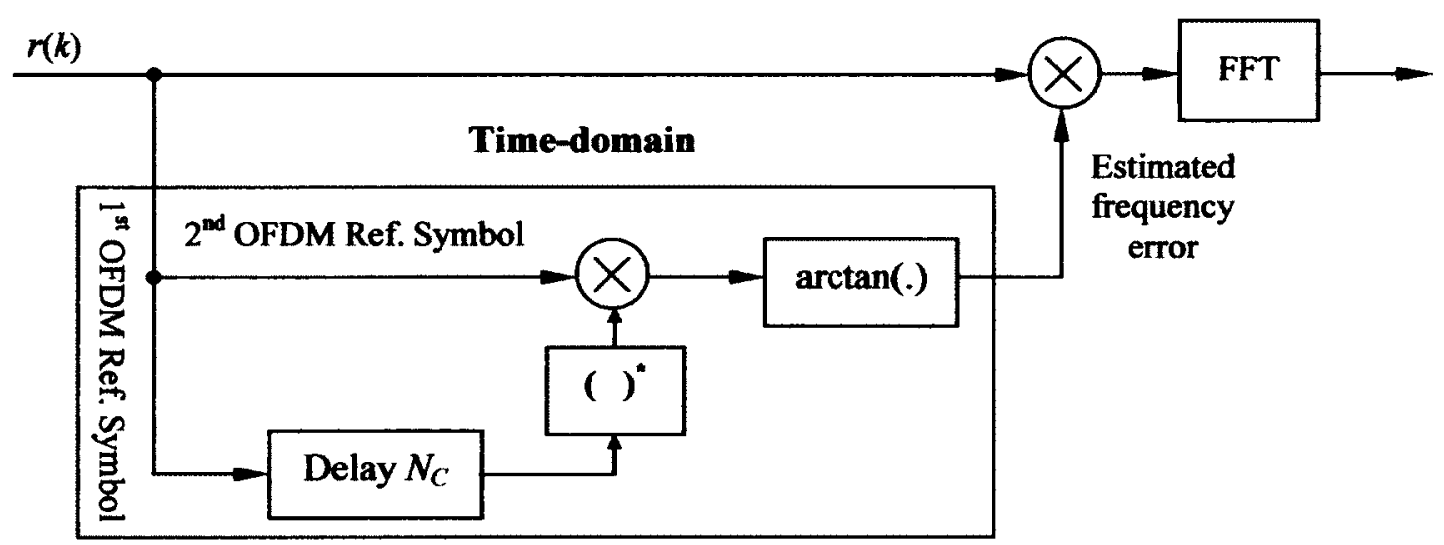

Figure 4.15. Frequency Offet Estimation using Maximum Likelihood

Schmidl \& Cox [6] proposed the CFO estimation method using a training symbol with two identical halves, whose estimation range is equal to \pm 1 sub-carrier spacing (Figure 4.16 [6]). The operating principle of this method is that two dedicated pilot symbols are used for synchronization. The first symbol contains null (zero) odd carriers and the second symbol has two interleaved PN sequences (odd/even carriers). The first symbol is used for timing and frequency estimation with a $2 / T$ ambiguity. The second symbol is used to remove the ambiguity on frequency estimation. 
In section 4.2 .2 a timing metric has been defined as:

$$
M_{\text {Schmidl }}(d)=\frac{|P(d)|^{2}}{(R(d))^{2}}
$$

Then a symbol timing estimate can be derived as:

$$
\hat{\imath}=\underset{d}{\arg }\{\max (M(d))\}
$$

And a carrier frequency offset estimate is:

$$
\hat{\varepsilon}=\frac{1}{\pi} \text { angle }\{P(d)\}
$$
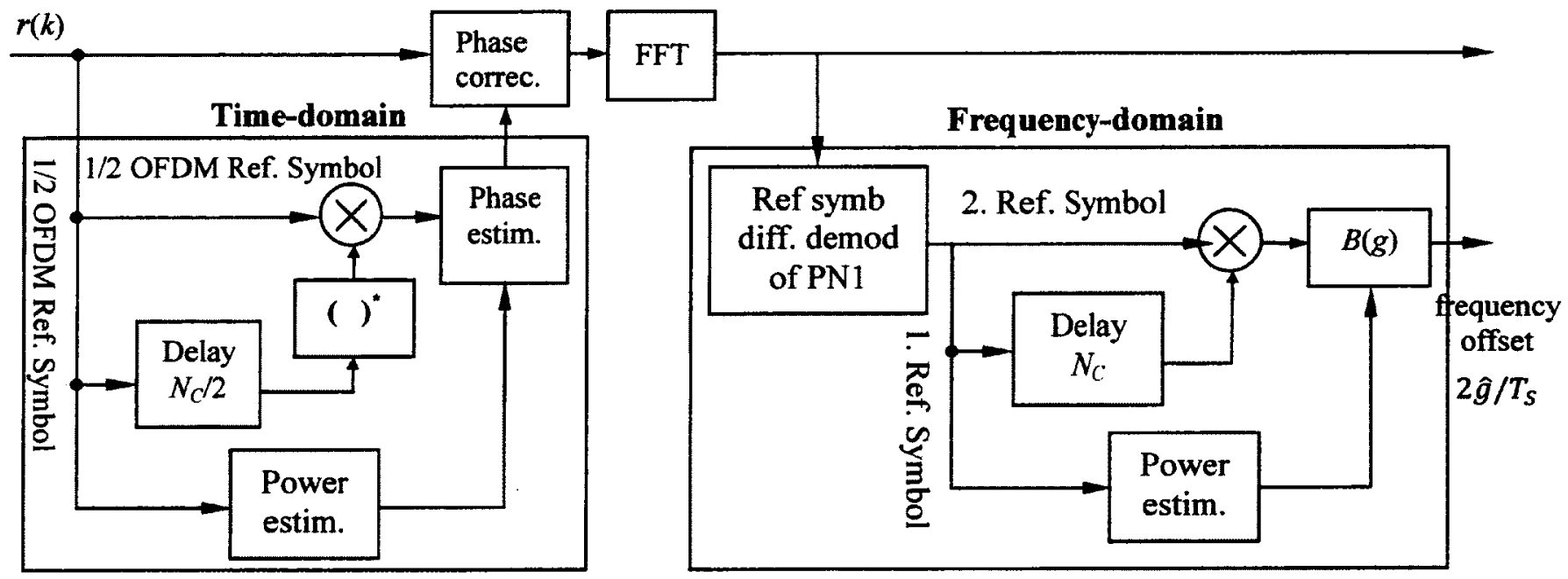

Figure 4.16. Frequency Offet Estimation using Two OFDM Symbols

Morelli \& Megali [25] impproved the SC method by using the best linear unbiased estimation (BLUE) principle. Their method uses only one training symbol, but consisting of many repeated parts. The estimation ragne is equal to $\pm \mathrm{L} / 2$ the sub-carrier spacing ( $L$ number of repeated parts). It improves CFO estimation range and accuracy but increases the required computation. In the absence of any frequency carrier offset (CFO), the best method for time synchronization is to calculate the correlation between a known reference and received sequence. However the presence of any frequency offset reduces the peak of the correlation function. In fact, this frequency offset prevents coherent addition of an individual term in the correlation calculation, and results in a drop of the correlation peak. 
Classen \& Meyr [21] have introduced a method which jointly finds both the STO and CFO. This is shown in Figure 4.17. However, this method is very complex computationally because it uses a trial and error technique where the CFO is incremented in small steps over the entire acquisition range until the correct carrier frequency is found. In the acquisition stage, it estimates a coarse/integer frequency estimation with a large acquisition range:

$$
\left.\hat{\varepsilon}_{\text {acq }}=\frac{1}{2 \pi T_{\text {sub }}} \underset{\varepsilon}{\operatorname{mag}}\left\{\mid \sum_{j=0}^{L-1} Y_{l+D}[p[j], \varepsilon] \cdot Y_{l}^{*}[p[j], \varepsilon] \cdot X_{l+D}^{*}[p[j]] \cdot X[p[j]]\right\}\right\}
$$

In the tracking stage, it locks and performs the tracking for the fine or fractional frequency estimation task, in which high tracking performance is required:

$$
\hat{\varepsilon}_{f}=\frac{1}{2 \pi T_{s u b} \cdot D} \arg \left\{\left|\sum_{j=0}^{L-1} Y_{l+D}\left[p[j], \hat{\varepsilon}_{a c q}\right] \cdot Y_{l}^{*}\left[p[j], \hat{\varepsilon}_{a c q}\right] \cdot X_{l+D}^{*}[p[j]] \cdot X[p[j]]\right|\right\}
$$

In the acquisition mode, $\hat{\varepsilon}_{a c q}$ and $\hat{\varepsilon}_{f}$ are estimated and then, the CFO is compensated by their sum. In the tracking mode, only $\hat{\varepsilon}_{f}$ is estimated and then compensated.

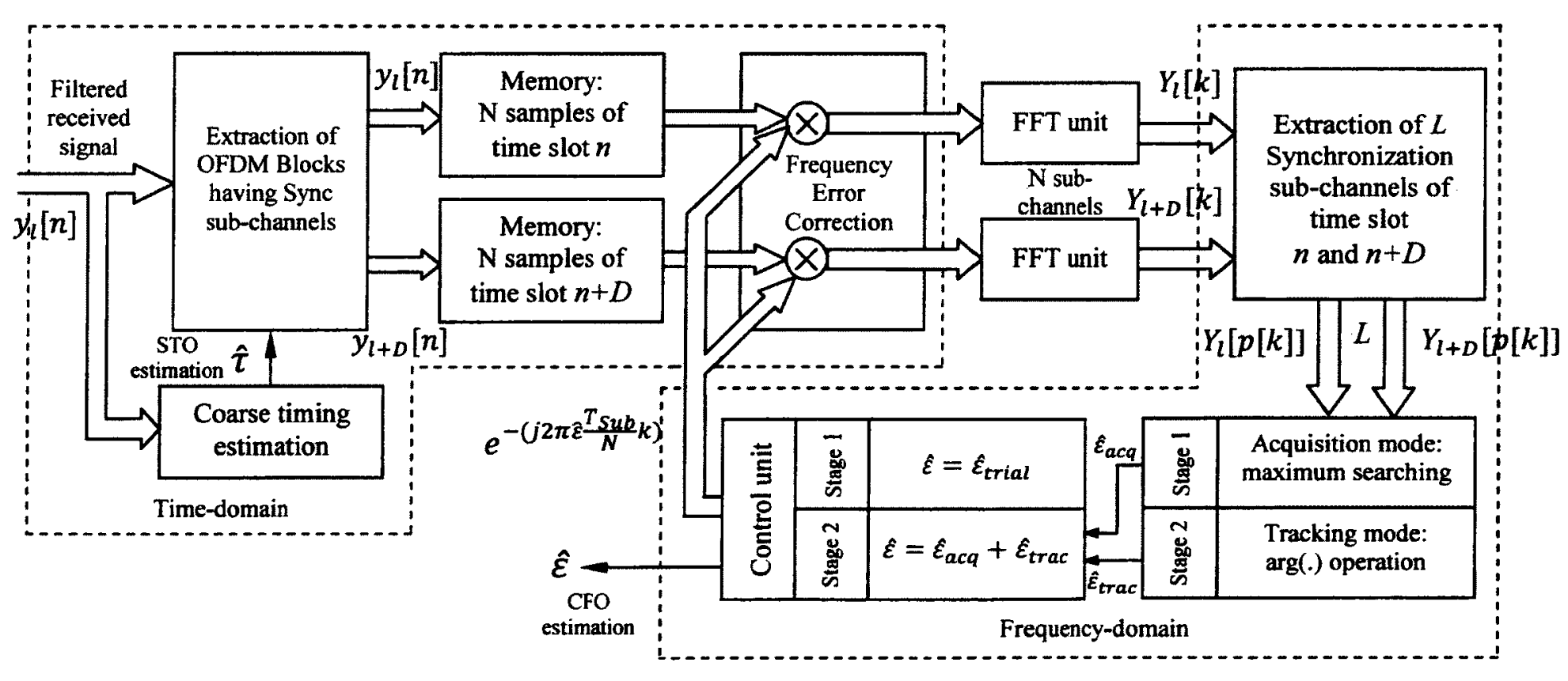

Figure 4.17. Data-Aided CFO Estimation using Pilot Tones [21] 


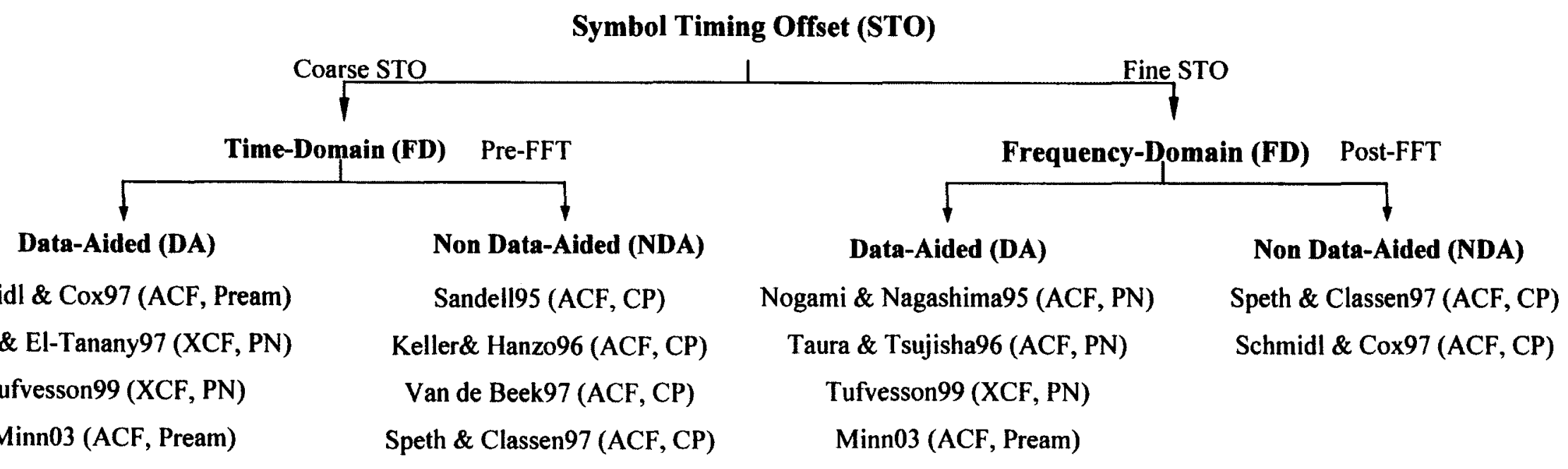

Shi \& Serpedin04 (ACF, Pream)

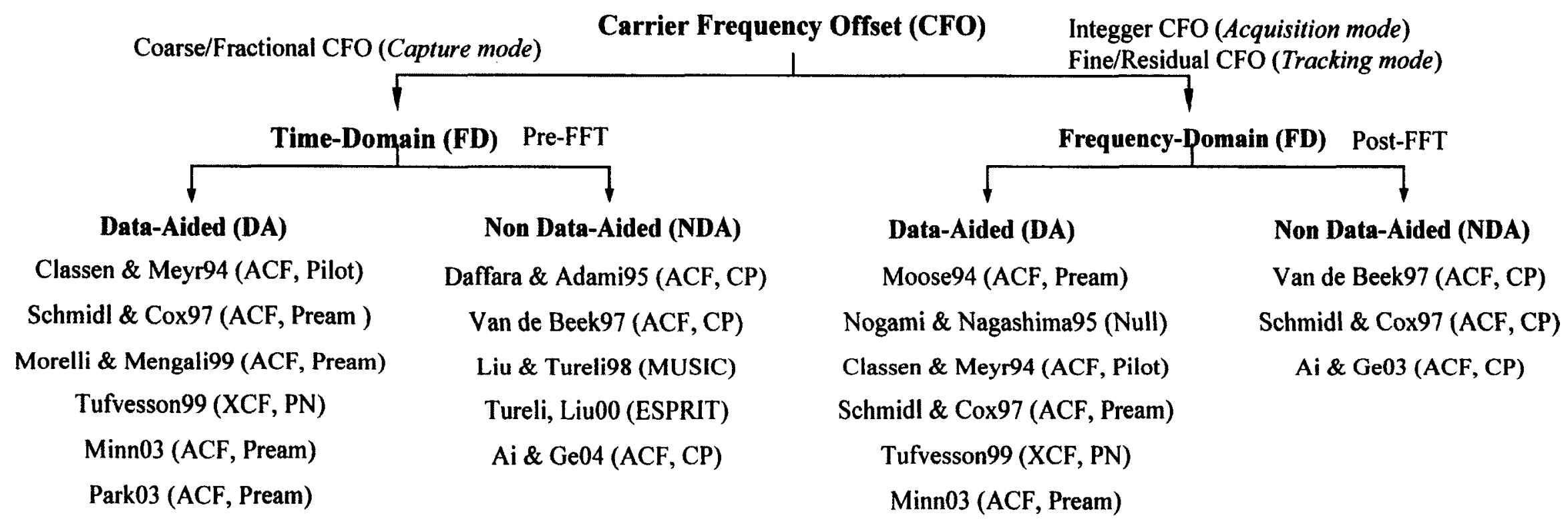

Figure 4.18. OFDM Time and Frequency Synchronization Techniques Classification

Figure 4.18 shows the classification of the latest research on OFDM time and frequency synchronization techniques. 


\subsection{Timing Offset and Frequency Offset Compensation Techniques}

The basic method to compensate for CFO offset $\varepsilon$ is to simply feedback the estimated CFO to the oscillator in the receiver. This can be accomplished by digitally multiplying each incoming sample by the exponential $e^{-j 2 \pi n \varepsilon T_{\text {sample }}}$. It is noted that the minus sign will counteract the rotation caused by the CFO.

While multipath induced phase rotations can be dealt with using differential encoding in OFDM communications, the loss of orthogonality due to carrier offset must be compensated for before discrete Fourier transform (DFT)-based demodulation can be performed.

If the timing offset $\tau$ is less than the cyclic prefix, then it will cause a phase rotation of $2 \pi k \Delta f \tau$ to the symbol at the $k^{\text {th }}$ subcarrier. If the timing offset exceeds the cyclic prefix, then ISI will be generated in addition to the phase rotation. The phase rotation due to the timing offset is different for different subcarriers.

After the STO is estimated, the STO integer part which is a multiple of the sampling interval is used to adjust the starting position of the FFT window, and the STO fractional (residual) part which generates a phase offset and can be compensated for at each subcarrier when we cancel the impact of the delay spread of wireless channels.

Once the CFO is estimated, the CFO integer part is a multiple of the subcarrier spacing $\Delta f$, which will cause a symbol or subcarrier shift, this means that the transmitted symbol in one subcarrier will be shifted to another at the receiver. The CFO fractional part results in the loss of orthogonality among the subcarriers which generates ICI. The CFO impact can be completely eliminated in the time-domain by multiplying the received signal by the frequency shift factor $e^{-j 2 \pi t}$. 


\subsection{LTE and LTE-Advanced Synchronization}

In LTE, the primary and secondary synchronization signals (P-SCH and S-SCH) provide acquisition of cell timing and identity during cell search. The downlink reference signal (RS) provides cell search, initial acquisition, coherent demodulation and channel estimation. The P-SCH and S-SCH signals are based on the Constant Amplitude Zero Autocorrelation (CAZAC) sequence. Constant amplitude is required to achieve a low peak-to-average power ratio (PAPR). Autocorrelation ensures good time-domain properties.

LTE cell identification (ID) is defined as: $N_{I D}^{\text {cell }}=3 N_{I D}^{(1)}+N_{I D}^{(2)}$, where $N_{I D}^{(1)}$ [33] is the physical layer identity group 1 carried by $\mathrm{S}-\mathrm{SCH}$, and $N_{I D}^{(2)}$ is the physical layer identity group 2 carried by $\mathrm{P}-\mathrm{SCH}$.

The P-SCH signal carries the physical layer identity $N_{I D}^{(2)}$, which is the cycle of three Zadoff-Chu sequences. One special CAZAC sequence is the Zadoff-Chu sequence, $\mathrm{P}-\mathrm{SCH}$ is a Zadoff-Chu sequence $\mathrm{d}_{\mathrm{u}}(n)$ using 3 different root indices according to the physical layer identity, where $u$ is the root index.

$$
d_{u}(n)=\left\{\begin{array}{lc}
e^{-j \frac{\pi u n(n+1)}{63}} & n=0,1, \ldots, 30 \\
e^{-j \frac{\pi u(n+1)(n+2)}{63}} & n=31,32, \ldots, 61
\end{array}\right.
$$

where $N_{I D}^{(2)}$ is defined as (Table $\left.4.1[33]\right)$ :

\begin{tabular}{|c|c|}
\hline$N_{10}^{(2)}$ & Root index $u$ \\
\hline 0 & 25 \\
\hline 1 & 29 \\
\hline 2 & 34 \\
\hline
\end{tabular}

Table 4.1. Root Indices for P-SCH

The 3 sequences based on these indices show the best auto-correlation characteristic from all the ZadoffChu sequences having this length, and simplify the synchronization procedure. They are transmitted on 62 of 72 reserved subcarriers or $6 \mathrm{RBs}$ (resource blocks) around the DC subcarrier, mapping to resource elements. The sequence $d(n)$ shall be mapped to the REs (resource elements) with frequency-domain index $k$ and time-domain index $l$, according to:

$$
a_{k, l}=d_{u}(n), \quad k=n-31+\left\lfloor\frac{N_{B W}^{D L} N_{S C}^{R B}}{2}\right\rfloor, \quad n=-5,-4, \ldots,-1,62,63, \ldots, 61
$$


where [.] represents the floor function, $N_{B W}^{D L}$ is the downlink bandwidth configuration, and $N_{S C}^{R B}$ is the resource block size in the frequency-domain.

S-SCH consists of two 31-bit BPSK M-sequence. S-SCH carries the physical layer cell identity group $N_{I D}^{(1)}[33]$

$$
\begin{gathered}
d(2 n)= \begin{cases}s_{0}^{\left(m_{0}\right)}(n) C_{0}(n) & \text { in subframe } 0 \\
s_{1}^{\left(m_{1}\right)}(n) C_{0}(n) & \text { in subframe } 5\end{cases} \\
d(2 n+1)= \begin{cases}s_{1}^{\left(m_{1}\right)}(n) C_{1}(n) z_{1}^{\left(m_{0}\right)}(n) & \text { in subframe } 0 \\
s_{0}^{\left(m_{0}\right)}(n) C_{1}(n) z_{1}^{\left(m_{1}\right)}(n) & \text { in subframe } 5\end{cases}
\end{gathered}
$$

where $0 \leq n \leq 30$.

The main goal of the P-SCH signal is to perform frame timing offset synchronization, coarse carrier frequency-offset detection and sector identification. Sector identification refers to slot timing and physical layer ID $(0,1,2)$ detection. The main goal of the S-SCH signal is to perform radio frame timing, cell ID, cyclic prefix length, FDD or TDD duplex detection.

In LTE, a subset of subcarriers for symbol \#6 in the slot $\# 0$ and slot $\# 10$ are designated as a primary synchronization channel (P-SCH). Furthermore, a subset of the subcarriers for symbol \#5 in the slot \#0 and slot \#10 are designated as the secondary synchronization channel 1 (S-SCH1) and secondary synchronization channel 2 (S-SCH2), respectively. The P-SCH and S-SCH are used to transmit the PSCH (Primary Synchronization Signal) and S-SCH (Secondary Synchronization Signal), respectively. As the first step of downlink synchronization in the 3GPP LTE system, the MS uses P-SCH with the period of $5 \mathrm{~ms}$, transmitted twice in a frame, to estimate symbol timing and $\mathrm{CFO}$. P-SCH is also used to detect the physical layer cell identity. As the second step of downlink synchronization, S-SCH is used to detect the physical layer cell ID group and frame timing. The LTE DL and UL are composed of physical channels and physical signals. Physical channels carry information from higher layers and are used to carry user data, as well as user control, and information. Physical signals do not carry information from higher layers and are used for cell search and channel estimation purposes only. 
The DL physical channels are the physical broadcast channel (PBCH), physical downlink control channel (PDCCH), physical hybrid ARQ indicator channel (PHICH), physical control format indicator channel (PCFICH) and physical downlink shared channel (PDSCH). The PDSCH Transmitting DL user data uses QPSK, 16QAM or 64QAM. The DL physical signals are the reference signal and the primary and secondary synchronization signals.

The UL physical channels are the physical uplink control channel (PUCCH), the physical uplink shared channel (PUSCH) and the physical uplink random access channel (PRACH). The PUSCH Transmitting UL user data uses QPSK, 16QAM or 64QAM. The PRACH carries random access preamble uses the Zadoff-Chu sequence. The UL physical signals are the sounding reference signal (SRS) and the demodulation reference signal for PUSCH and PUCCH.

For an LTE-OFDM symbol with pilots (i.e., Primary Synchronization Signal Zadoff-Chu CAZAC sequence), if the tone numbering is correct, $\left(k_{0}=0\right)$, then the demodulated symbols at pilot position will have a high cross-correlation with the known pilots. Otherwise, the cross-correlation is very low [19]. It is noted that, even though LTE introduce scalable bandwidth scheme with different FFT/IFFT point, the sub-carrier spacing is constant. Therefore, we can only use the 2048-pojnt FFT instead of all possibilities.

A Zadoff-Chu sequence is a complex-valued mathematical sequence which, when applied to radio signals, gives rise to an electromagnetic signal of constant amplitude, whereby cyclically shifted versions of the sequence imposed on a signal result in zero cross-correlation with one another at the receiver. A generated Zadoff-Chu sequence that has not been shifted is known as a "root sequence". The sequence then exhibits the useful property that cyclically shifted versions of itself are orthogonal to one another, provided, that is, that each cyclic shift, when viewed within the time domain of the signal, is greater than the combined propagation delay and multi-path delay-spread of that signal between the transmitter and receiver. 
Zadoff-Chu CAZAC Sequences Properties:

1) If $N_{\mathrm{ZC}}$ is prime, the Zadoff-Chu sequences are periodic with period $N_{\mathrm{ZC}}$.

$$
x_{u}\left(n+N_{Z C}\right)=x_{u}(n)
$$

2) If $N_{\mathrm{ZC}}$ is prime, DFT of a Zadoff-Chu sequence is another Zadoff-Chu sequence conjugated, scaled and time scaled (where $\tilde{u}$ is the multiplicative inverse of $u$ modulo $N_{Z C}$ ).

$$
X_{u}(k)=x_{u}^{*}(\tilde{u} k) X_{u}(0)
$$

3) The autocorrelation of a prime length Zadoff-Chu sequence with a cyclically shifted version of itself also has zero auto-correlation (i.e., it is non-zero only at one instant which corresponds to the cyclic shift).

4) The cross correlation between two prime length Zadoff-Chu sequences is constant $1 / \sqrt{N_{Z C}}$ (i.e. different $u$ ).

5) The Zadoff-Chu sequences have low PAPR.

Figure 4.19 (adapted from [36]) shows the proposed LTE/LTE-Advanced OFDM synchronization process. The coarse time offset estimate $\hat{\boldsymbol{\tau}}$ and coarse frequency offset estimate $\hat{\varepsilon}$ are computed based on the received LTE-OFDM I/Q waveform sequence $y(n)$. The frequency offset estimate $\hat{\varepsilon}$ is used to counter-rotate $y(n)$ at an angular speed $2 \pi \hat{\varepsilon} / \mathrm{N}$ (coarse frequency correction), while the timing estimate $\hat{t}$ is used to find the correct positioning of the receive DFT window (coarse timing correction).

In the proposed algorithm, two OFDM symbols $y_{l}[\mathrm{n}]$ and $y_{l+D}[\mathrm{n}]$, are saved in memory after synchronization. Then, the signals are transformed into $Y_{[}[k]$ and $Y_{1+D}[k]$ via FFT, from which pilot tones are extracted. After estimating CFO from the pilot tones in the frequency-domain, the signal is compensated with the estimated CFO in the time-domain. Pilots are placed every $D^{\text {th }}$ OFDM symbols. In this process, two different estimation modes for the CFO estimation are implemented: the acquisition and tracking modes. In the acquisition mode, a large range of CFO including an integer CFO is estimated. 


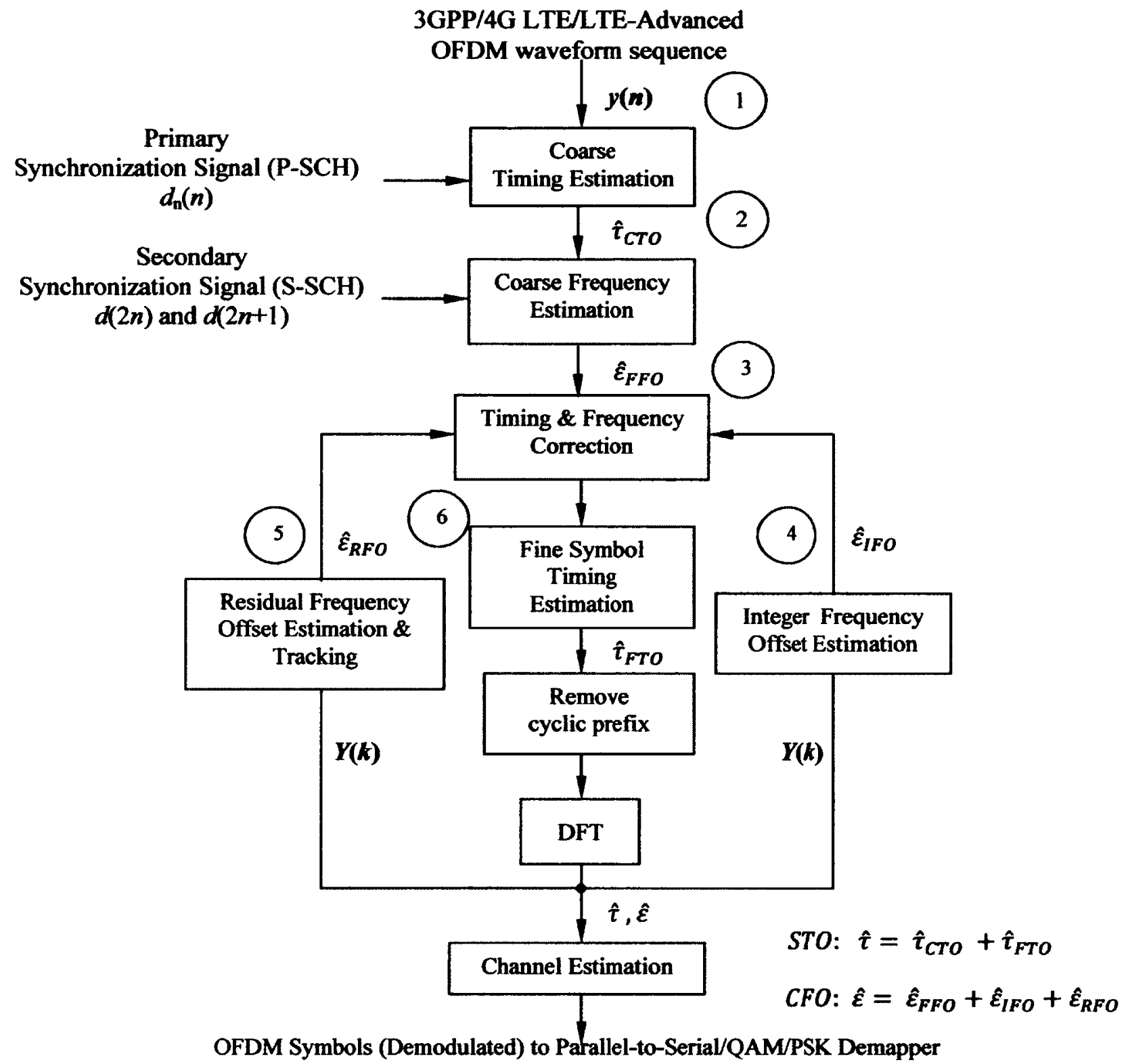

Figure 4.19. Proposed LTE/LTE-A OFDM Synchronization using Zadoff-Chu CAZAC

The main idea of the proposed LTE-OFDM algorithm frequency synchronization is to divide the carrier frequency offset (CFO) into a Fractional Frequency Offset (FFO), an Integer Frequency Offset (IFO), and a Residual Frequency Offset (RFO), which can be estimated individually. The coarse or fractional frequency offset is performed in the time-domain, whereas the integer frequency offset (IFO) and fine or residual frequency offset (RFO) are estimated in the frequency-domain. A complete LTE-OFDM synchronization symbol timing offset (STO: $\left.\tau_{\mathrm{STO}}=\tau_{\mathrm{CTO}}+\tau_{\mathrm{FTO}}\right)$ and carrier frequency offset (CFO: $\varepsilon_{\mathrm{CFO}}=$ $\left.\varepsilon_{\mathrm{FFO}}+\varepsilon_{\mathrm{FO}}+\varepsilon_{\mathrm{RFO}}\right)$ estimation process flowchart in shown in Figure 4.19. 
Figure 4.20 shows the location of the primary (P-SCH) and secondary (S-SCH) synchronization signals in a 10ms LTE frame. P-SCH and S-SCH are inserted into the last two OFDM symbols in the first slot of the sub-frame zero and sub-frame five. The P-SCH signal is transmitted twice in each $10 \mathrm{~ms}$ frame and can provide frame timing synchronization with a tolerance of $5 \mathrm{~ms}$. Figure 4.21 (a) and (b) shows the matched filtering synchronization using the primary signal (P-SCH) in time-domain and frequency-domain respectively [34].

LTE-OFDM Radio frame $=10 \mathrm{~ms}$

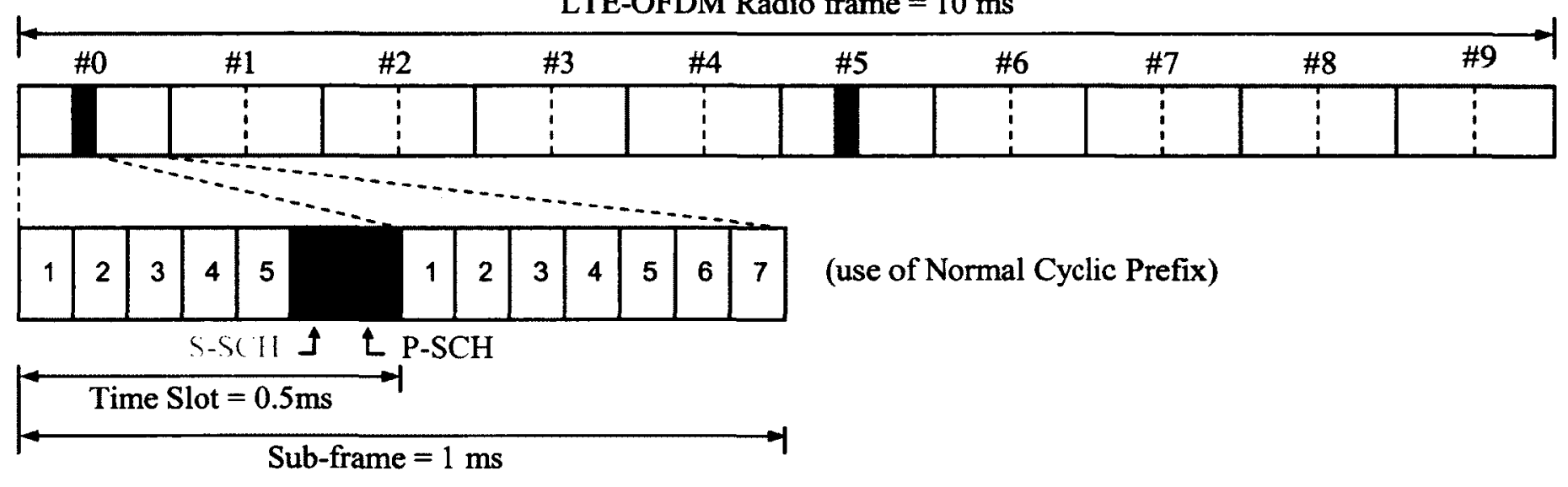

Figure 4.20. P-SCH and S-SCH Signals Location in LTE-OFDM Radio Frame

In order to compensate for frequency selective channels Classen \& Meyr94 [21] have used as a pilot signal a principle similar to a differential encoded pseudo-noise PN sequence. The novelty of the proposed algorithm is an application of a known technique by Classen \& Meyr94 [21] to a new area of LTE/LTE-Advanced. In contrast to [21], which is based on a differentially encoded PN sequence, the proposed algorithm is based on a generated pseudorandom polyphase Zadoff-Chu CAZAC sequence.

Figure 4.21(a) shows that prior to frequency domain processing of $\mathrm{P}-\mathrm{SCH}$, an initial coarse synchronization is needed in order to determine an initial position of the DFT window [34]. This initial estimation can be extracted either from the CP of the downlink signal, or from the time-domain-matched filtering with the IDFT of the Zadoff-Chu sequence. Figure 4.21(b) shows that once coarse synchronization has been performed in the time domain, CFO estimation can be accurately obtained from matched filtering in the frequency-domain [34]. 


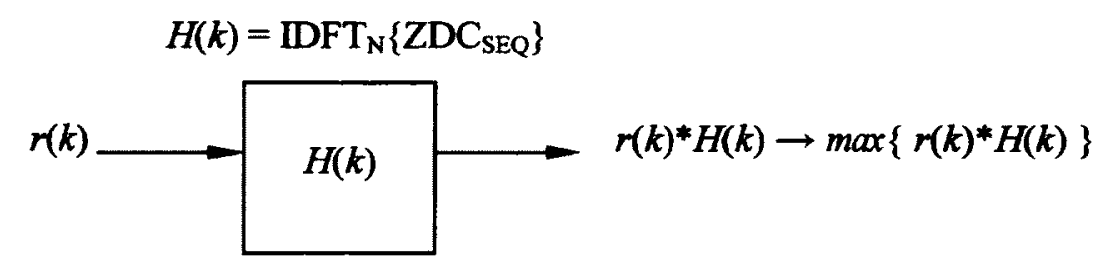

(a) Time-Domain Approach

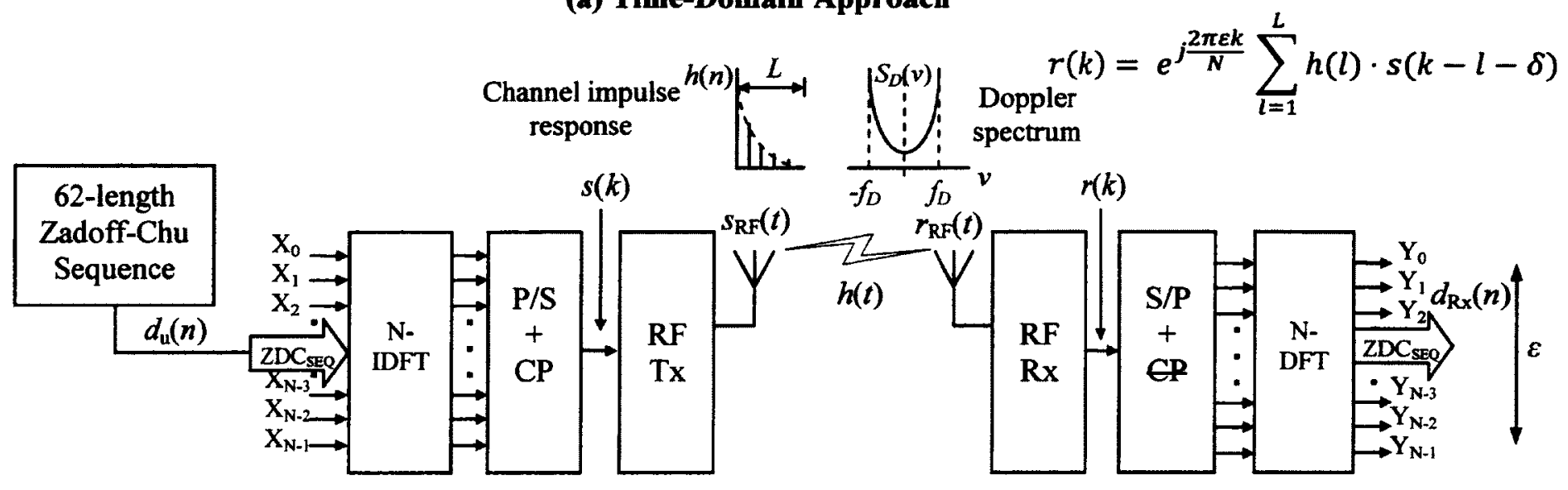

(b) Frequency-Domain Approach

Figure 4.21. Analysis of Zadoff-Chu CAZAC Matched Filtering LTE-OFDM Synchronization [34]

In Figure 4.22, there are two main steps related to the proposed LTE-OFDM synchronization using CAZAC sequence: symbol synchronization, or time synchronization and frequency synchronization.

In Figure 4.22(a), the portion of the cyclic prefix corrupted by ISI depends on the length of the channel response, but, in general, a valid range of values exists for acquiring time synchronization inside the ISI safe zone. If the value of $\hat{t}$ is located within this region, data subcarriers, $X_{k}$ will not be affected by ISI, but rather by a phase shift proportional to $k$ index and $\Delta \hat{\tau}$. This phase shift causes a rotation of the received subcarriers in the I/Q plane and can be easily compensated in the frequency-domain. However, for values of $\hat{\tau}$ located outside the ISI safe zone, the DFT window will be corrupted by the contiguous symbol, causing dispersion on the received subcarriers and degrading the error rate [34]. In Figure 4.22(b), in the case of an imperfect frequency correction of $\hat{\varepsilon}$, the time domain received signal after CFO compensation is multiplied by a complex residual carrier; this leads to a shift in the frequency domain of the received subcarriers, $Y_{k}$, resulting in the loss of orthogonality among all subcarriers. 
Time Synchronization
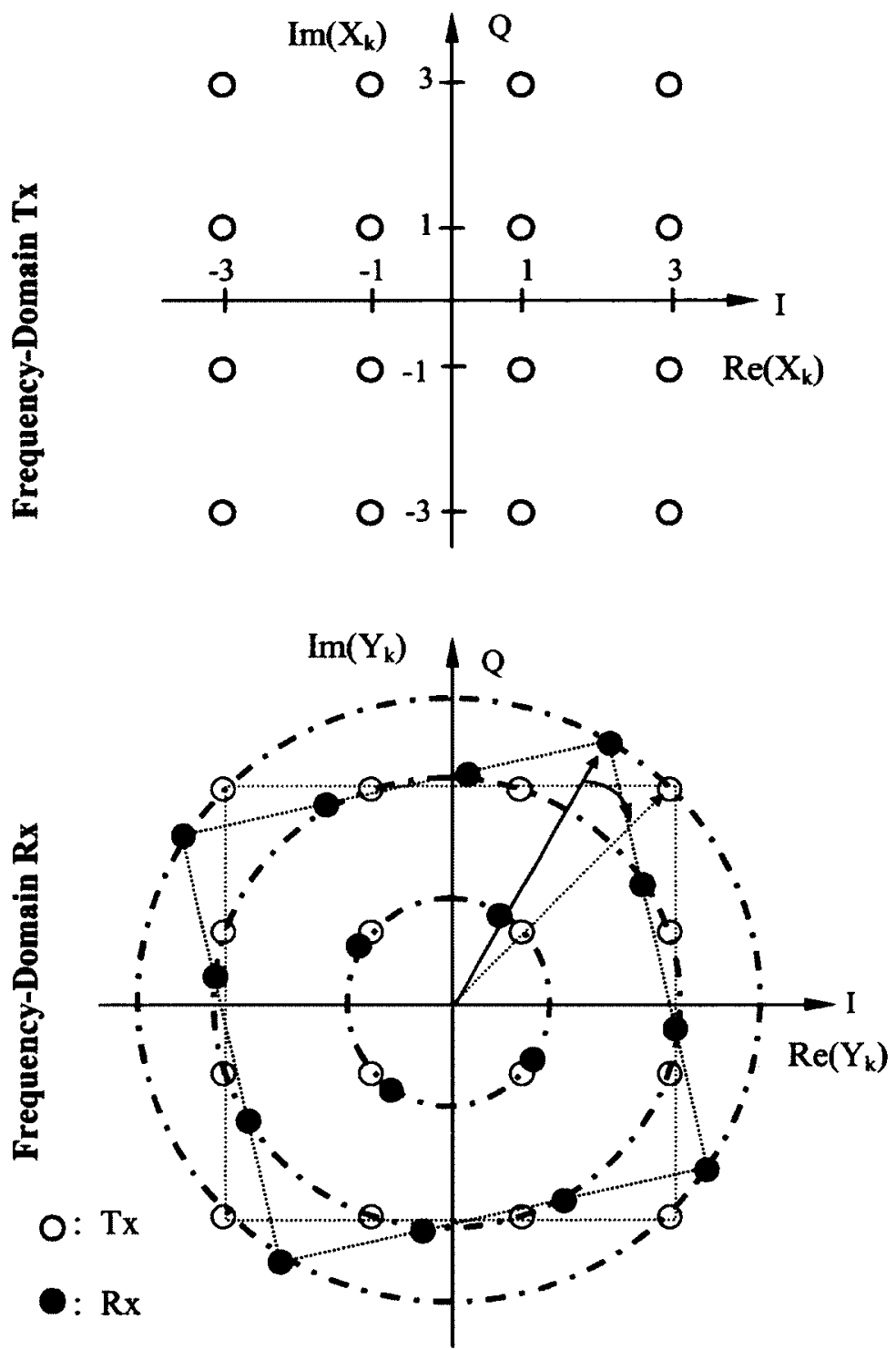

Frequency Synchronization
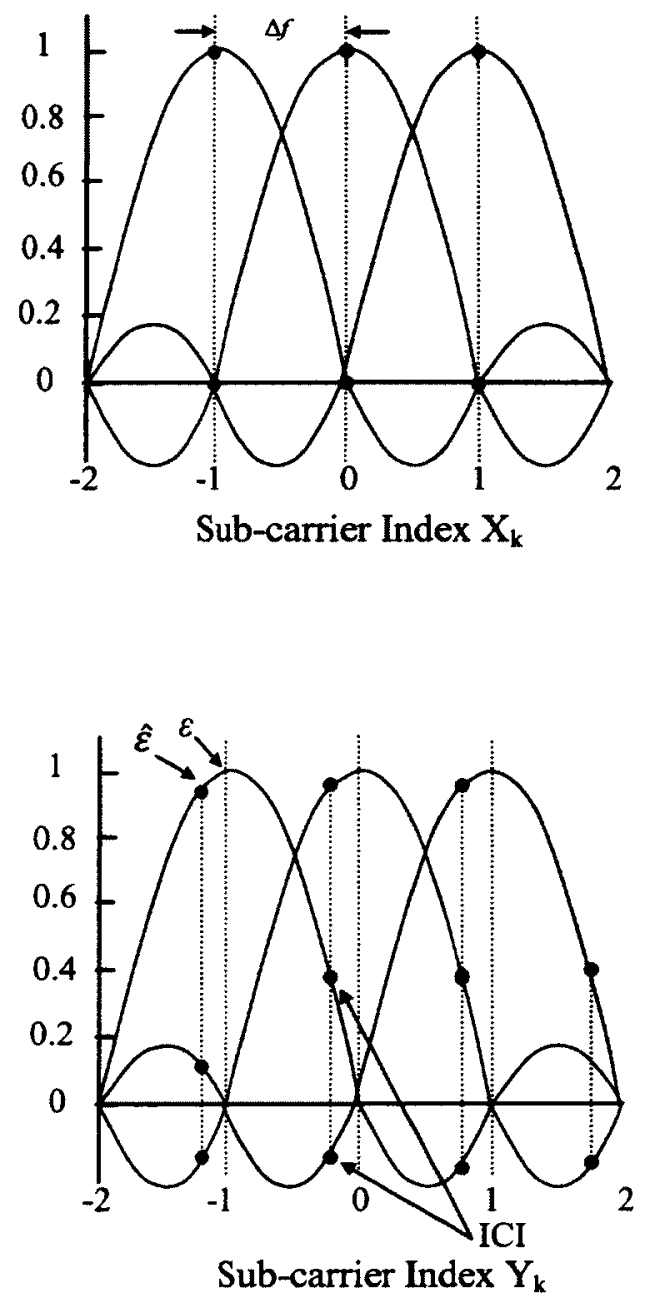

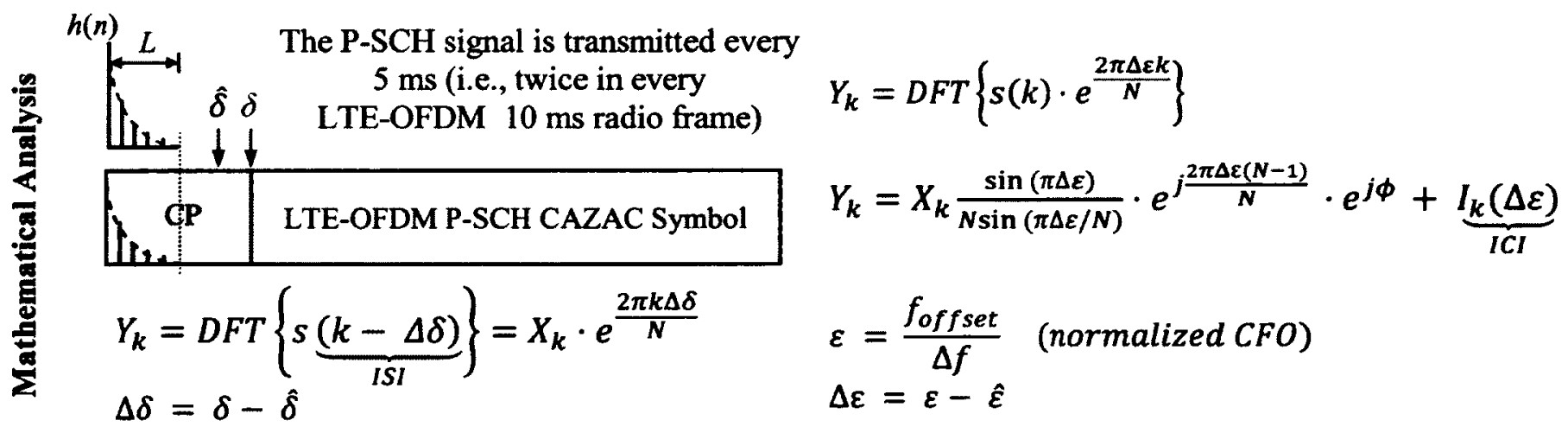

(a)

(b)

Figure 4.22. Analysis of LTE-OFDM Time and Frequency Synchronization using CAZAC Sequence 


\subsection{Modulation Efíiciency and Synchronization Performance Measurement}

This section discusses the performance and efficiency measurement methods of the LTE modulation and synchronization algorithms as well as other parameters that also influence the algorithm performance.

In digital communications, we often use $E_{\mathrm{b}} / N_{0}$, a normalized version of SNR, as a figure of merit. An energy signal is defined as a signal having finite energy but zero average power. A power signal is defined as a signal having finite average power and infinite energy. Therefore, power is a more useful parameter for characterizing an analog signal, whereas energy is more appropriate to characterize digital signal. For digital systems, a figure of merit should allow us to compare one system with another at the bit level. Therefore, a description of the digital waveform in terms of $\mathrm{S} / \mathrm{N}$ is virtually useless, since the waveform may have a one-bit meaning, a two-bit meaning, or a 10-bit meaning [37]. One of the most important metrics of performance in digital communications systems is a plot of the bit-error probability $P_{B}$ versus $E_{b} / N_{0}$. Since $E_{b}=\mathrm{S} \cdot \mathrm{Tb}=S / R_{\mathrm{b}}$ and $N_{0}=N / W$, we have:

$$
\frac{E_{b}}{N_{0}}=\frac{S}{N}\left(\frac{W}{R}\right)
$$

where $\quad E_{\mathrm{b}}$ : is the bit energy; $N_{0}:$ is the noise power spectral density;

$S:$ is the signal power; $N:$ is the Noise power, $W:$ is the noise bandwidth; $R$ : is the bit rate

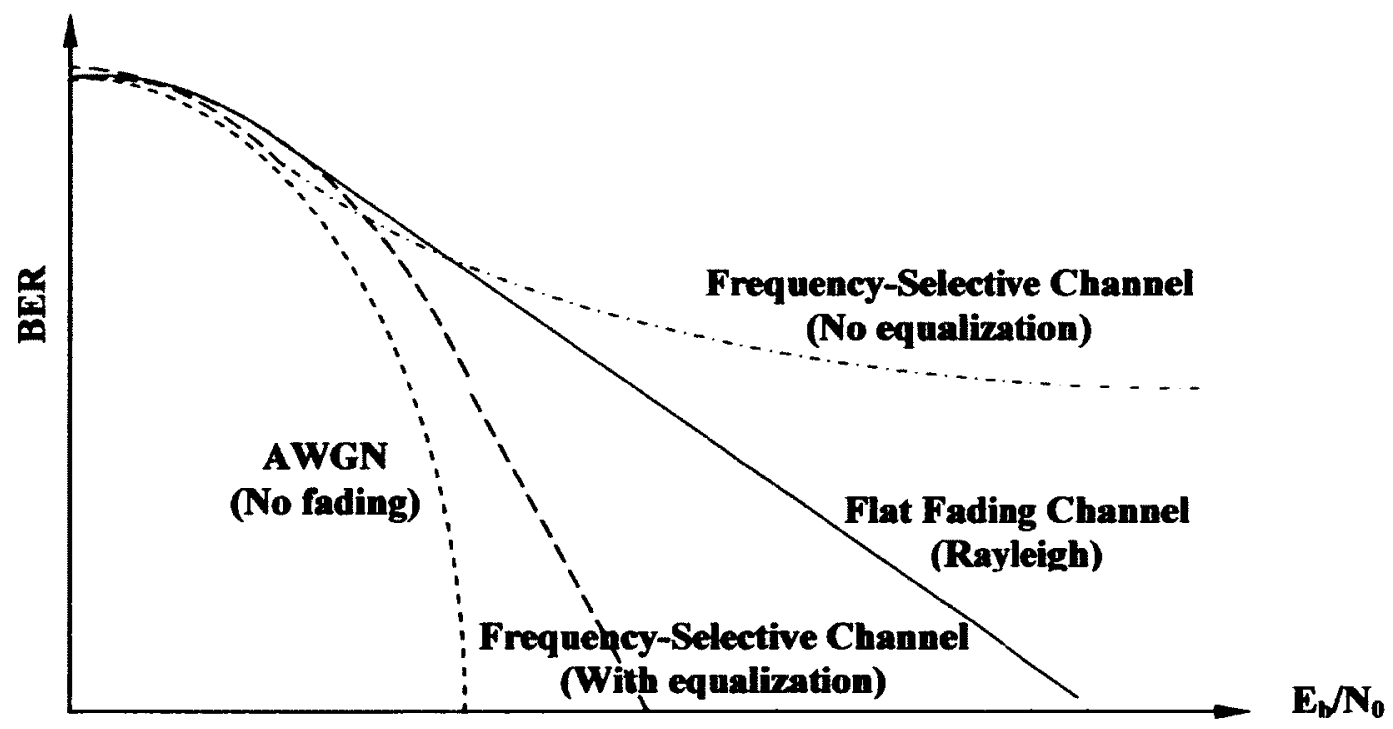

Figure 4.23. BER vs. Eb/NO Performance of LTE-OFDM Modulation over Wireless Channel 
Figure $\cdot 4.24$ shows the theoretical BER vs. $E_{\mathrm{b}} / N_{0}$ performance over AWGN and a Rayleigh channel for frequency-selective fading with and without equalization. From equation 4.5 we can observed that as the signal power $\mathrm{S}$ increases, the bit energy over the noise energy $E_{\mathrm{b}} / N_{0}$ increases. As the noise power $N$ increases, the bit energy over the noise energy $E_{\mathrm{b}} / N_{0}$ decreases. As the signal bandwidth $\mathrm{W}$ increases, the bit energy over the noise energy $E_{\mathrm{b}} / N_{0}$ increases. As the signal bit rate $R_{b}$ increases, the bit energy over the noise energy $E_{\mathrm{b}} / N_{0}$ decreases. In multipath Rayleigh fading channel, there is one or more major reflected paths from Tx to Rx. In multipath Rician fading channel, there is only direct line-of-sight path from transmitter to receiver.

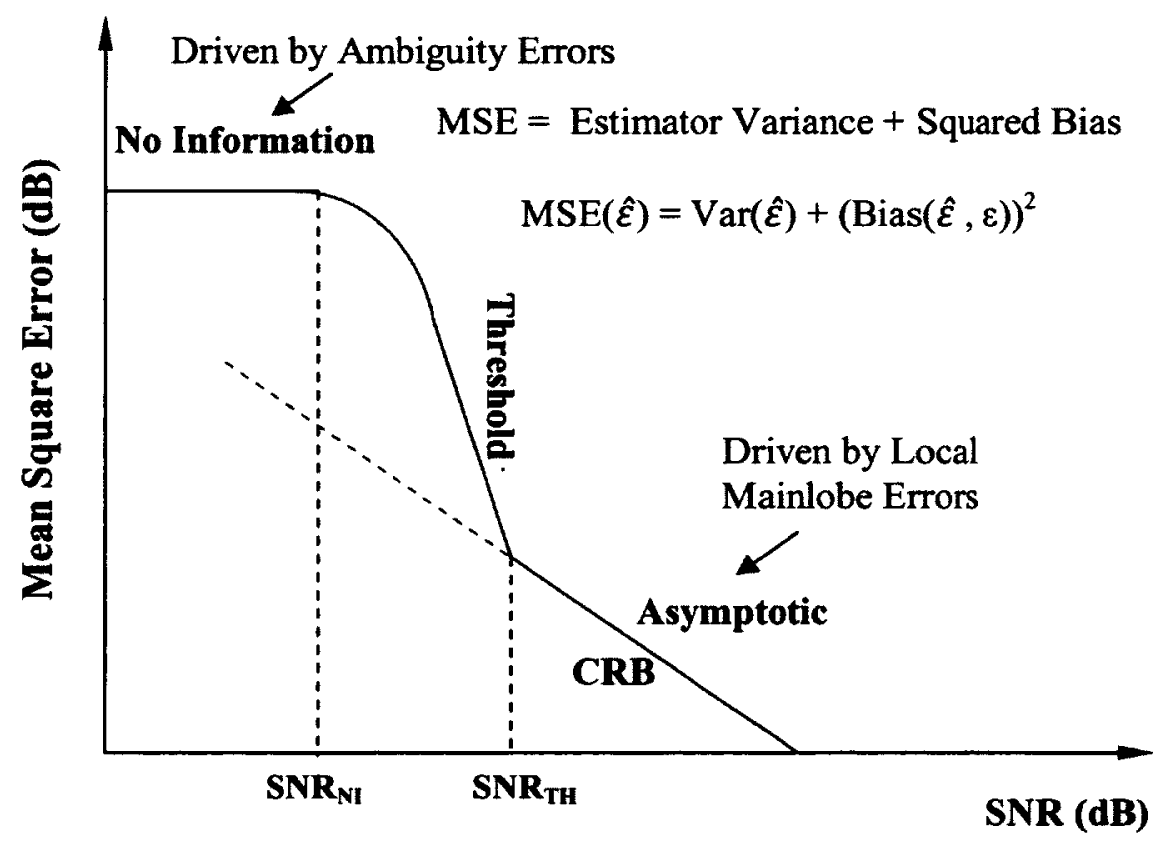

Figure 4.24. MSE vs. SNR Performance of LTE-OFDM Synchronization over Wireless Channel

Figure 4.24 shows the theoretical MSE (Mean Square Error) vs. SNR performance behavioural areas. In MSE versus SNR performance, the main objective is to get a good prediction of the $\mathrm{SNR}_{\mathrm{TH}}$ (Threshold) and reasonable prediction of the $\mathrm{SNR}_{\mathrm{NI}}$ (No Information). The MSE is equal to the sum of the variance and the squared bias of the estimator: $\operatorname{MSE}(\hat{\varepsilon})=\operatorname{Var}(\hat{\varepsilon})+(\operatorname{Bias}(\hat{\varepsilon}, \varepsilon))^{2}$. Theoretically, the mean squared error (MSE) of an estimator is one of many metrics to quantify the difference between values implied by 
an estimator $\hat{\varepsilon}$ and the true values of the quantity being estimated $\varepsilon$. In other words, MSE measures the average mean square deviation of the estimator from the true value.

$$
\begin{aligned}
\operatorname{MSE}(\hat{\varepsilon})=E\left\{(\hat{\varepsilon}-\varepsilon)^{2}\right\}= & E[(\hat{\varepsilon}-E(\hat{\varepsilon}))+(E(\hat{\varepsilon})-\varepsilon)]^{2}=\operatorname{Var}(\hat{\varepsilon})+E\{\hat{\varepsilon}-\varepsilon\}^{2} \\
& M S E(\hat{\varepsilon})=\operatorname{Var}(\hat{\varepsilon})+B^{2}(\hat{\varepsilon})
\end{aligned}
$$

where $\hat{\varepsilon}$ is the estimator with respect to the estimated parameter $\varepsilon$, which shows that the MSE is composed of errors due to the variance of the estimator as well as the squared bias.

The above figure of merit BER/SER vs. $E_{b} / N_{0}$ and the Mean Square Error (MSE) vs. SNR estimator method will be used for performance efficiency evaluation of LTE-OFDM modulation schemes as well as the performance efficiency of the proposed LTE-OFDM synchronization algorithm using the Zadoff-Chu CAZAC pilot in Chapter 5. That is, LTE-OFDM modulation schemes for 2-QAM /BPSK (1 bit/s/Hz), 4QAM /QPSK (2 bit/s/Hz), 16-QAM (4 bit/s/Hz), 64-QAM (6 bit/s/Hz), 256-QAM (8 bit/s/Hz) performance efficiency will be experimentally examined and simulated against other synchronization methods. In addition, in Chapter 5 the performance efficiency of the proposed LTE-OFDM synchronization algorithm using the Zadoff-Chu CAZAC pilot sequence (frequency-domain) will be experimentally tested against other synchronization algorithm methods such as cyclic prefix-based method (time-domain) and preamble-based method (frequency-domain).

In addition, the performance of any synchronization algorithm is determined by parameters such as: the minimum SNR under which the operation of synchronization is guaranteed, the acquisition time and range (max tolerable deviation range of timing offset, LO frequency), the overhead in terms of the reduced data rate (due to cyclic prefix, preamble, pilot, etc.), complexity, robustness and accuracy in the presence of multipath and interferences. 


\subsection{Conclusion}

This chapter has presented an overview, classified, and analyzed several major OFDM synchronization techniques. An OFDM synchronization method for LTE/LTE-Advanced based on an enhancement of Classen's technique [21] has been developed and proposed. However, unlike Classen's single carrier differential decoding sequence, a Zadoff-Chu CAZAC sequence is used as a pilot multicarrier training data.

The Zadoff-Chu sequence is known as a CAZAC sequence (Constant Amplitude Zero Auto Correlation waveform). CAZAC codes are a class of complex-valued pseudo-random noise sequences with a cyclic autocorrelation equal to zero, and has been widely used in wireless systems. Zadoff-Chu sequences are used in the 3GPP LTE Long Term Evolution air interface in the Primary Synchronization Signal (P-SCH) (so-called primary synchronization channel), random access preamble (PRACH), HARQ ACK/NACK responses (PUCCH) and sounding reference signals (SRS). By assigning orthogonal Zadoff-Chu sequences to each LTE eNodeB and multiplying their transmissions by their respective codes, the crosscorrelation of simultaneous eNodeB transmissions is reduced, thus reducing intra-cell interference and uniquely identifying eNodeB transmissions. The Zadoff-Chu sequence improvement over the WalshHadamard codes is used in UMTS because they result in a constant-amplitude output signal, reducing the cost and complexity of the radio's power amplifier.

The problem of symbol or time synchronization in an OFDM-based system is focused on finding the instant at which the OFDM symbol starts (estimation is denoted as $\hat{\imath}$ ). On the other hand, the problem of frequency synchronization is related to the correction of the CFO (estimation is denoted as $\hat{\varepsilon}$ ) that affects the received signal.

Modulation efficiency and algorithm performance measurements methods as well as parameters that influence the algorithm performance have been presented and discussed. 


\section{Chapter 5 Experimental Results and Discussions}

\subsection{Introduction}

In this chapter, a proposed algorithm is developed to implement the LTE/LTE-Advanced OFDM synchronization and estimations techniques discussed in Chapter 4. First, the LTE-OFDM baseband signal is configured and generated using Aeroflex IQCreator ${ }^{\mathrm{TM}}$ ARB software. Then the LTE baseband waveform is downloaded into an Aeroflex IFR 3416 signal generator (Transmitter) where the signal is upconverted and modulated to form the LTE-OFDM FDD RF signal. Next, the LTE RF signal is received and down-converted into baseband with a Rohde \& Schwarz R\&S ${ }^{\circledR}$ FSV spectrum analyzer (Receiver). The LTE-OFDM baseband BB signal is then captured and saved in an I/Q format file using the CRC Spectrum Explorer ${ }^{\circledR}$ spectrum monitoring software. Finally, the LTE baseband I/Q waveform file is loaded into Matlab where the signal is digitally processed by the LTE synchronization algorithm in which symbol timing and carrier frequency offset estimations are obtained.

\subsection{Waveforms Generation, Transmission, and Reception Setup}

In order to replicate the LTE-OFDM realm of waveform creation, transmission, and reception, we need to use a combination of four very sophisticated and specialized software packages and hardware instruments: IQCreator ${ }^{\mathrm{TM}}$ (for waveform creation), Aeroflex IFR 3416 (for RF transmission), R\&S FSV (for BB reception), and CRC Spectrum Explorer (for I/Q waveform capture). IQCreator ${ }^{\mathrm{TM}}$ is a powerful Windows ${ }^{\mathrm{TM}}$ based software tool that provides all the necessary features required for a quick and easy method to create a modulation scheme from which an ARB (Arbitrary Waveform Generator) complex waveform can be generated. Waveforms can be created that conform to the LTE standards regarding timing, spectral distribution and amplitude probability distribution. Graphical displays of the waveform FFT, vector and constellation diagrams, etc. can be viewed and exported for use in other Windows ${ }^{\mathrm{TM}}$ applications such as Matlab. The resultant waveform can then be downloaded to the Aeroflex 3416 Series ARB signal generator. 
IQCreator $^{\mathrm{TM}}$ provides the capability to simulate LTE systems in accordance with 3GPP Release 8 Version 8.5 in the FDD transmission format. IQCreator supports the multiple access schemes followed by LTE for the physical layer which is based on orthogonal frequency division multiplexing (OFDM) for the downlink (DL), and single carrier frequency division multiple access (SC-FDMA) for the uplink (UL). IQCreator ${ }^{\mathrm{TM}}$ is capable of supporting the different LTE bandwidth settings $1.4 \mathrm{MHz}, 3 \mathrm{MHz}, 5 \mathrm{MHz}, 10$ $\mathrm{MHz}$ and $20 \mathrm{MHz}$ in accordance with the standard. The default full carrier allocation can be overridden by customizing the resource block to the carrier level. Additionally, the parameters for the physical channels and physical signals can be configured on a frame and subframe basis. In particular the PDSCH channel modulation can be set to be QPSK, QAM16 or QAM64. Further testing flexibility is allowed by setting up different data sources to any of the uplink or downlink channels. Choices are available for using the PRBS, all zeros, all ones, random bits, repeating patterns, or any type of user data from a file.

The Aeroflex IFR 3410 Series signal generator is capable of producing high quality digitally modulated carriers with RF modulation bandwidths of up to $25 \mathrm{MHz}$. An LTE waveform is packaged and downloaded to the Aeroflex IFR 3410 Series ARB signal generator to modulate the LTE waveform.

The Rohde \& Schwarz FSV spectrum analyzer ensures high measurement accuracy. It has a $28 \mathrm{MHz}$ signal analysis bandwidth with a base unit (40 MHz optional), 16-bit $\mathrm{A} / \mathrm{D}$ converter with $128 \mathrm{MHz}$ sampling frequency giving wide dynamic range, excellent display linearity, and a 200 Msample signal memory. In the standard configuration, the R\&S FSV provides an internal I/Q memory in order to capture and evaluate $\mathrm{I} / \mathrm{Q}$ data, which can be output via the LAN interface. The A/D converter samples the IF signal at a rate of $128 \mathrm{MHz}$. The digital signal is downconverted to the complex baseband, lowpassfiltered, and decimated (the sample rate is reduced). The continuously adjustable sampling rate is realized using an optimal decimation filter and subsequent resampling at the set sample rate. The $\mathrm{I} / \mathrm{Q}$ data is written to a single memory. The data acquisition is hardware-triggered. The maximum number of samples for the RF input is 200 MSamples. 
Figure 5.1 shows the analyzer hardware from the IF section to the processor. This block diagram illustrates the I/Q data signal processing of the LTE RF input, i.e., in the I/Q Analyzer of the R\&S FSV. The A/D converter samples the IF signal at a rate of $128 \mathrm{MHz}$. The digital signal is downconverted to the complex baseband, lowpass-filtered, and the sampling rate reduced. The continuously adjustable sample rates are realized using an optimal decimation filter and subsequent resampling at the set sample rate. The I/Q data is written to a single memory. The data acquisition is hardware-triggered. The maximum number of samples for RF input is 200MSamples. After downconversion, the LTE BB signal is captured by the CRC Spectrum Explorer and saved in the I/Q format.

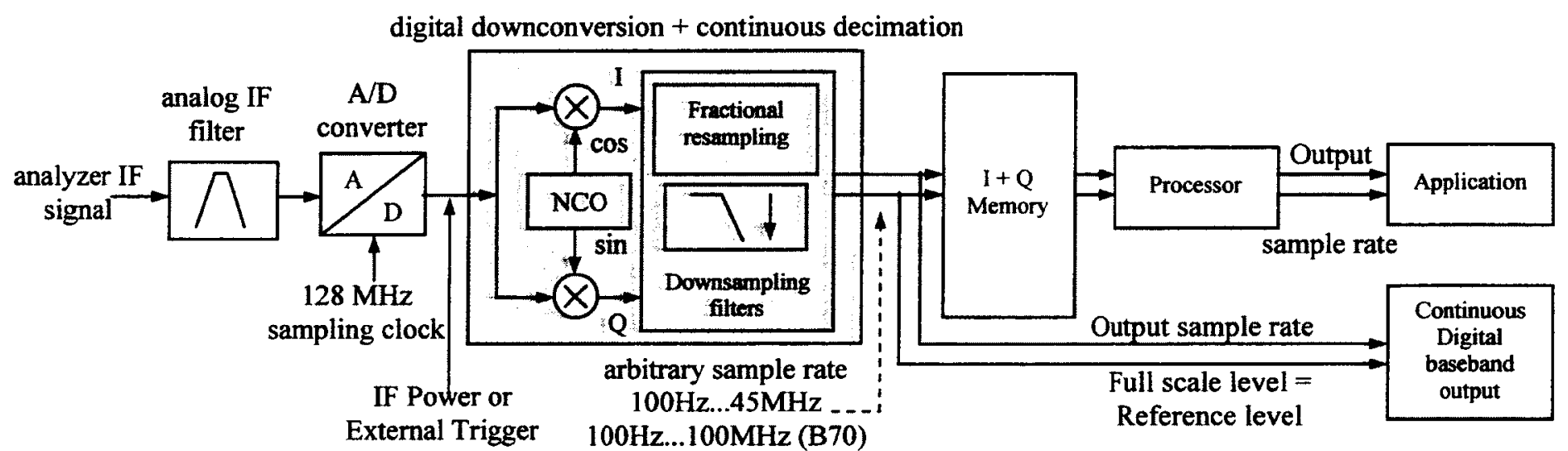

Figure 5.1. Digital Acquisition Hardware Receiver (R\&S FSV) Block Diagram

The CRC Spectrum Explorer ${ }^{\circledR}$ (SE) is a flexible software tool that performs spectrum surveillance and analysis. Installed on a Windows-based PC, SE works with a broad range of commercial RF receiving and digitizing hardware. CRC SE provides the digital signal processing and user-friendly GUI control needed by specialists to assess the usage and quality of the radio spectrum. CRC Spectrum Explorer ${ }^{\circledR}$ core routines consist of CRC Wideband Scanner (WBS) and CRC Spectrum Analyzer (SA) software. The CRC Spectrum Analyzer (SA) provides narrow band spectrum scanning and a dynamic display of power spectral density versus frequency. In this research, the LTE complex downconverted I/Q signal waveform file is then loaded into Matlab for symbol timing and frequency offset estimations using the proof of concept STO/CFO synchronization algorithm code. 


\subsection{Spectrum Sensing Performance Results}

This section describes the spectrum sensing performance assessment using the Receiver Operating Characteristics (ROC) curves and the Probability of Occurrence (POC) curves. Receiver Operating Characteristics (ROC) curves present graphical summaries of a detector's performance and give the probability of detection $\left(P_{D}\right)$ for a given probability of false alarm $\left(P_{F A}\right)$.

Figure 5.2 and 5.3 show the receiver operating characteristics (ROC) curves trade-off between probability of detection $\boldsymbol{P}_{D}$ and probability of false alarm $\boldsymbol{P}_{F A}$ for different number of sensors, in linear scale and logarithmic scale respectively. The graphs show that as the number of sensors increases the supports of the probability distributions PDFs under the null $\left(\mathrm{H}_{0}\right)$ and alternative $\left(\mathrm{H}_{1}\right)$ hypotheses become more disjoint. That is, when there are more sensors ( 5 sensors) the sensing performance improves and when there are less sensors ( 3 sensors) the sensing performance degrades. When the signal is stronger there is less overlap (i.e., the noise and signal + noise are further apart) in the POC curves, and the ROC curve becomes more bowed. Figure 5.3 plots the same data on a logarithmic scale for $P_{F A}$, which better reveals the characteristics of the ROC for $P_{F A}$ of interest in spectrum sensing signal processing.

Figure 5.4 and 5.5 show Probability of Occurrence (POC) curves $\mathrm{P}_{\mathrm{D}}$ and $\mathrm{P}_{\mathrm{FA}}$ Tradeoff. Although the proportion between $P_{F A}$ and $P_{D}$ can be adjusted via threshold control, it is not possible to simultaneously attain a low $P_{F A}$ and high $P_{D}$. In order to enhance the sensing performance, the distance between two PDFs needs to be lengthened or the PDF of each hypothesis should be steepened. The first way to improve the performance the detection/false-alarm trade-off is to increase the SNR. Figure 5.4 illustrates the effect of increased SNR by causing the two PDFs to move further apart and the performance probabilities under the two hypotheses using the same detection threshold. The second way to improve the performance trade-off is to reduce the overlap of the PDFs by reducing their variance. Figure 5.5 shows the effect of reduced noise power causing the two PDFs to move closer to one another using the same detection threshold. 


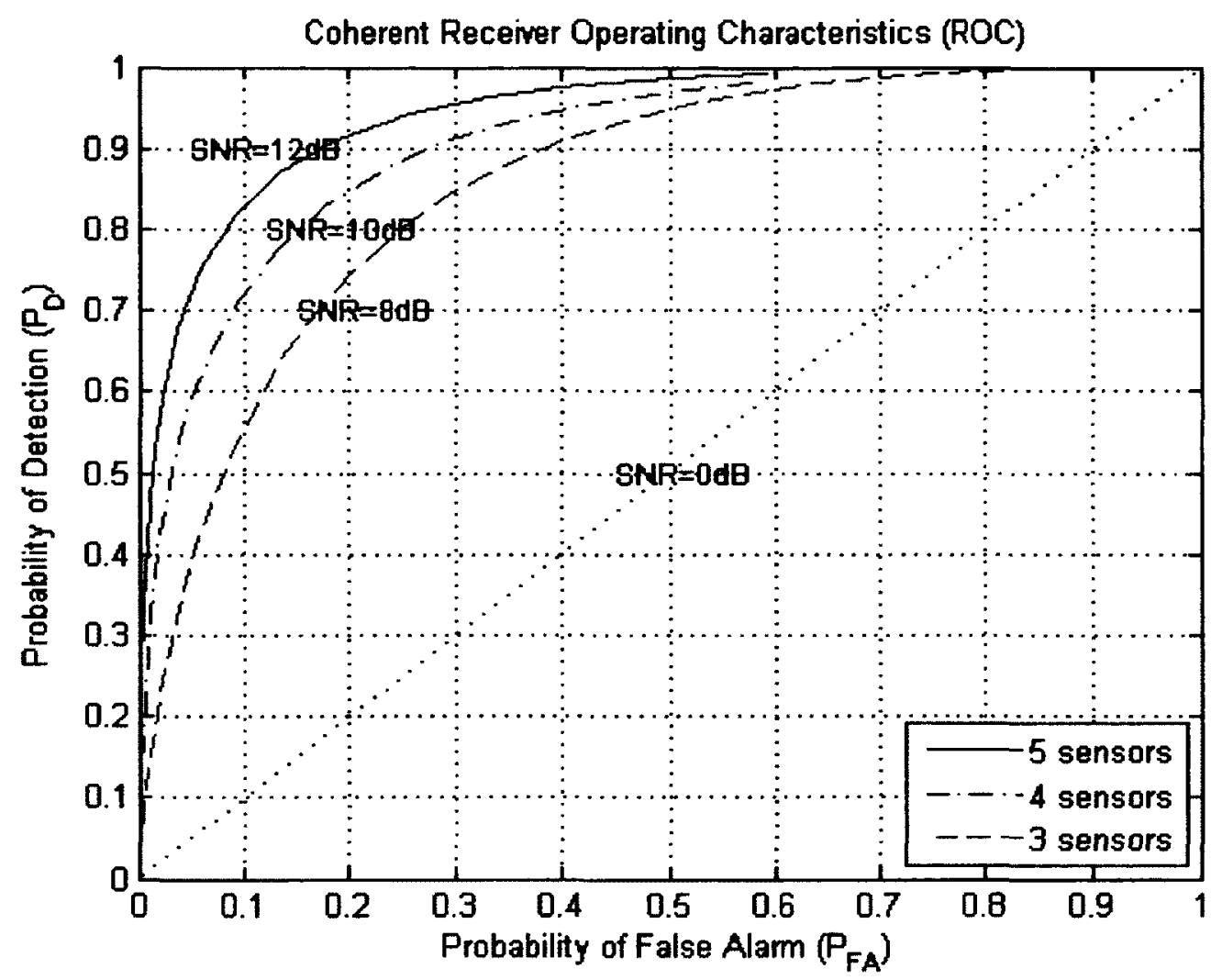

Figure 5.2. Receiver Operating Characteristics (ROC) $P_{D}$ vs. $P_{F A}$ Linear Scale

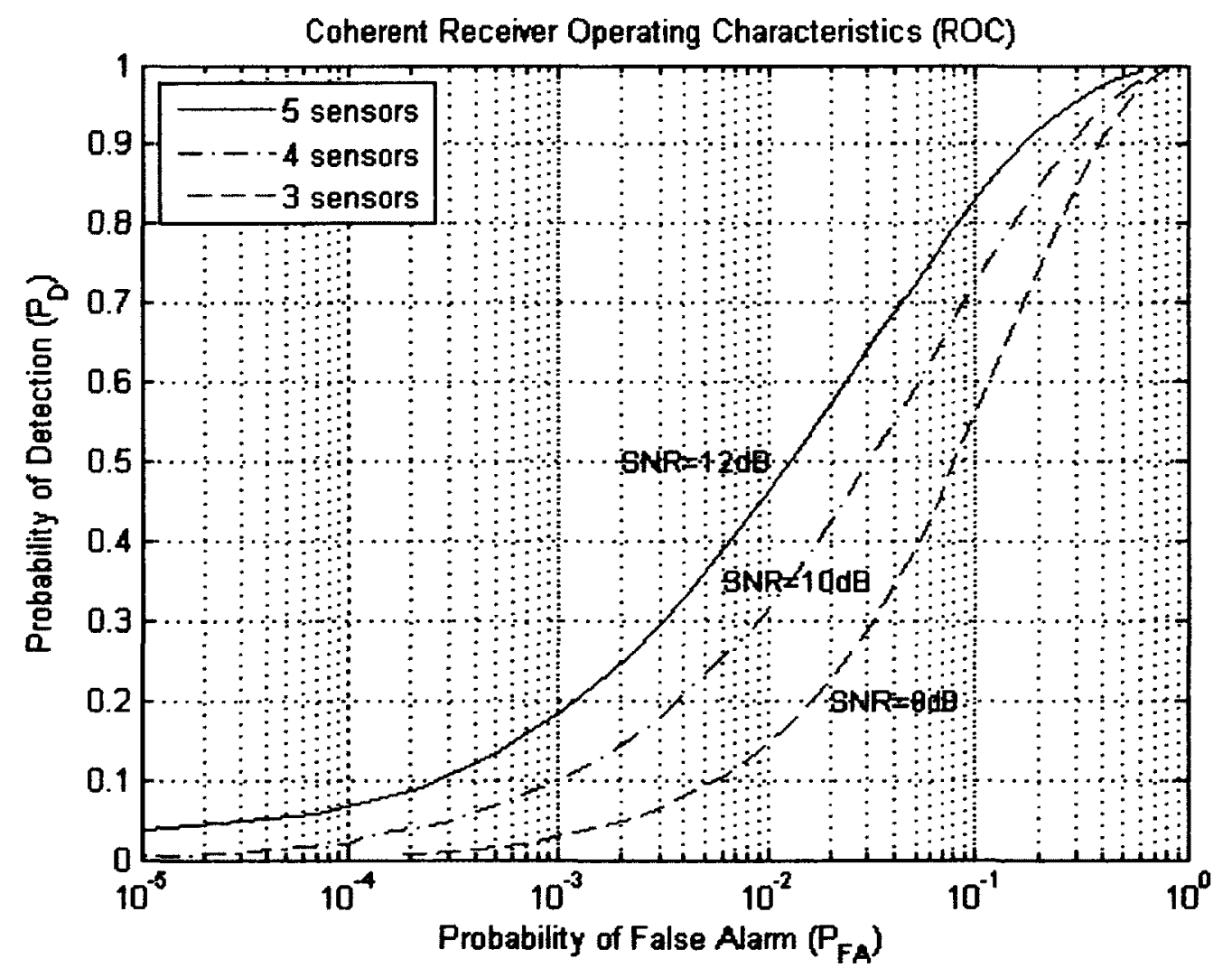

Figure 5.3. Receiver Operating Characteristics (ROC) $P_{D}$ vs. $P_{F A}$ Logarithmic Scale 


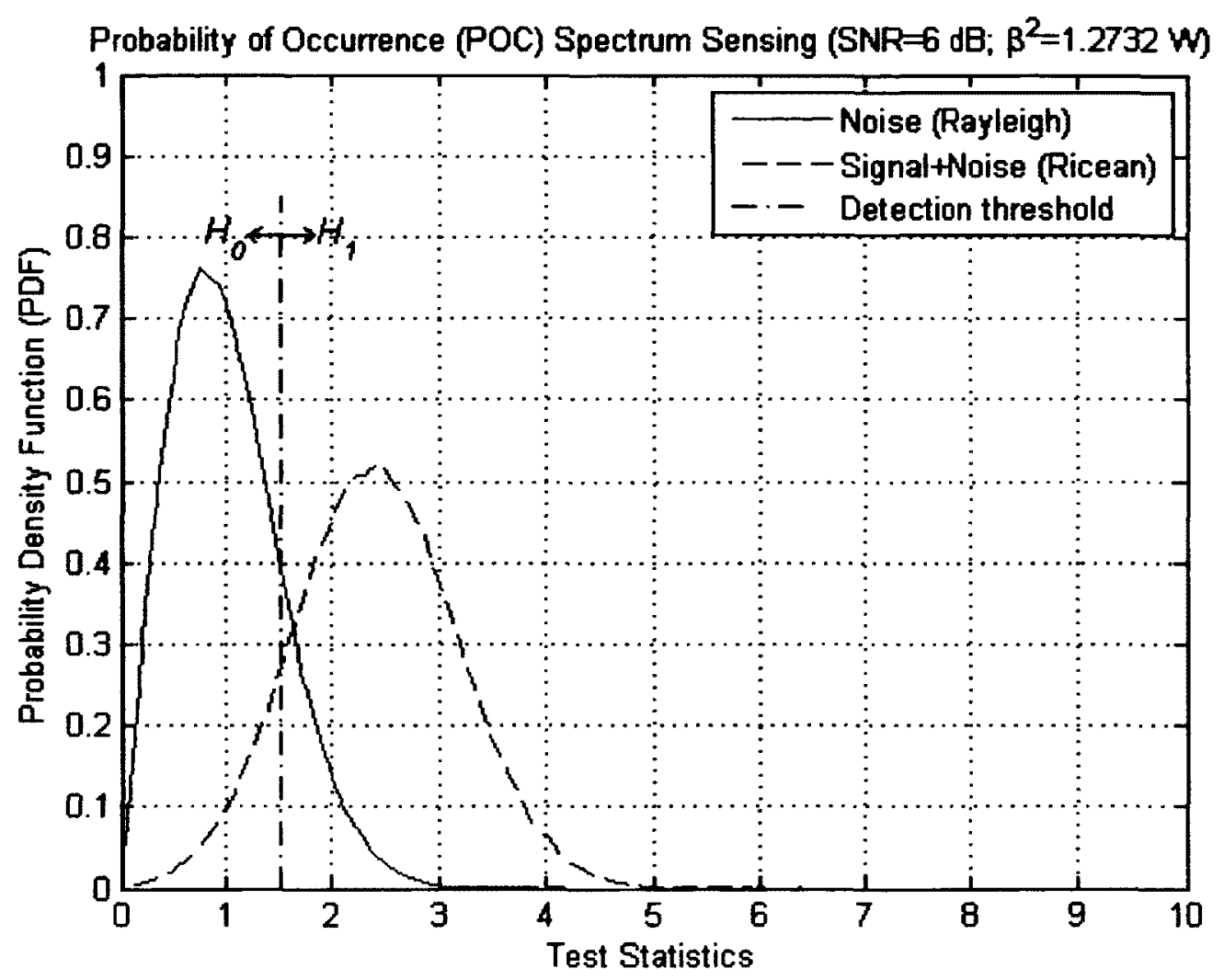

(a) Low SNR

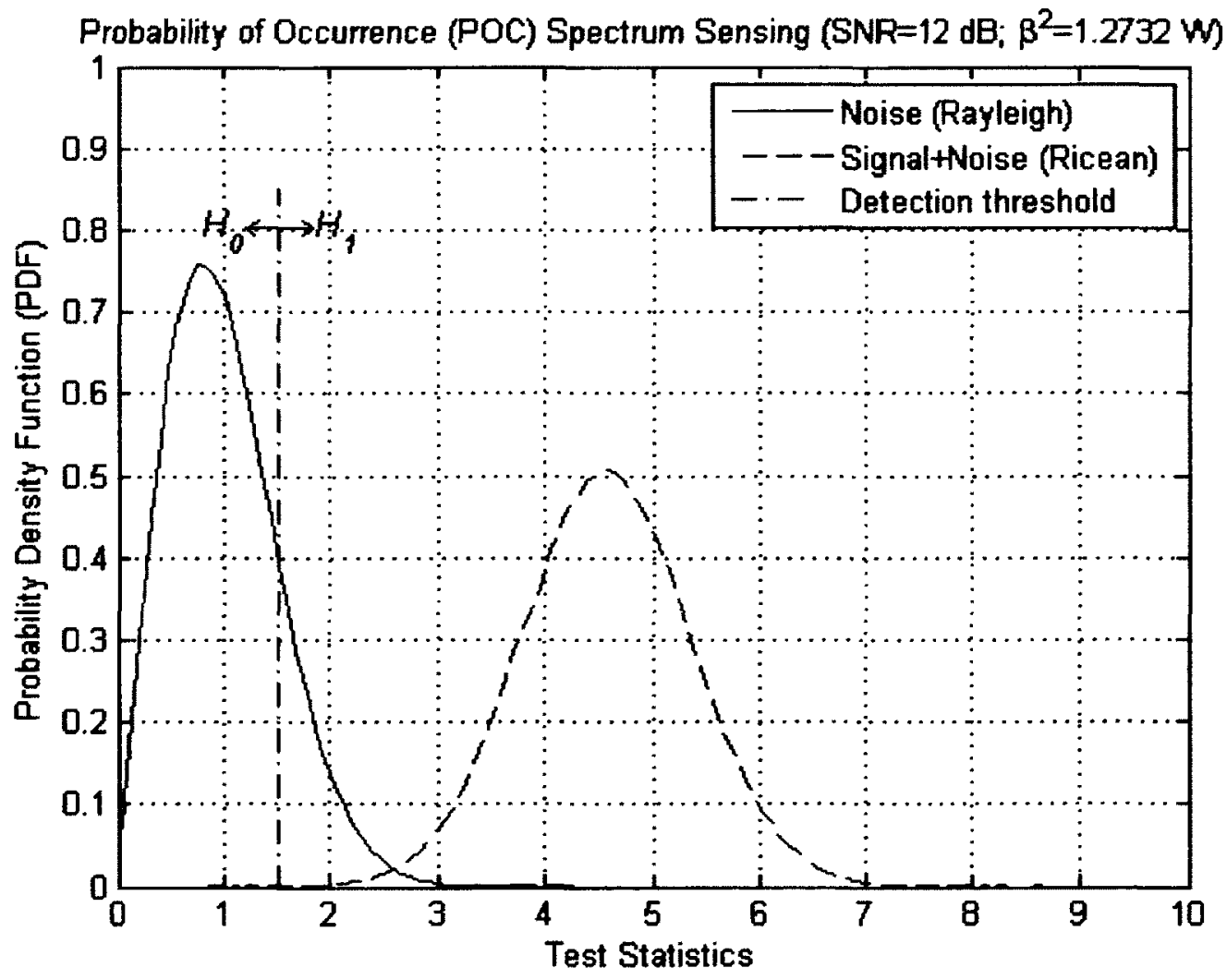

(b) High SNR

Figure 5.4 Probability of Occurrence (POC) $P_{D}$ and $P_{F A}$ Tradeoff by Increasing the $S N R$ 
Probability of Occurrence (POC) Spectrum Sensing (SNR=9dB; $\beta^{2}=1.2732 \mathrm{~W}$ )

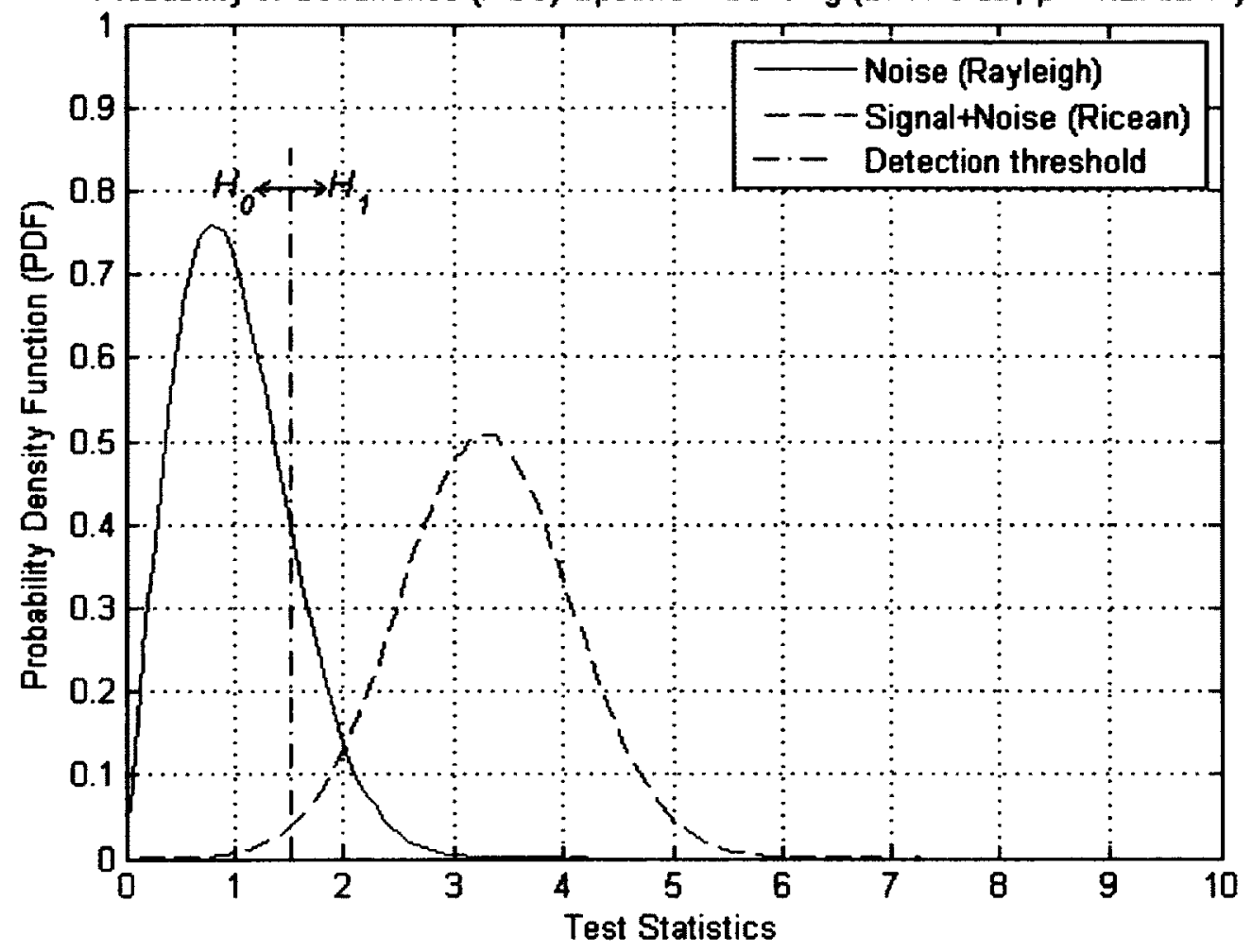

(a) High Noise Power

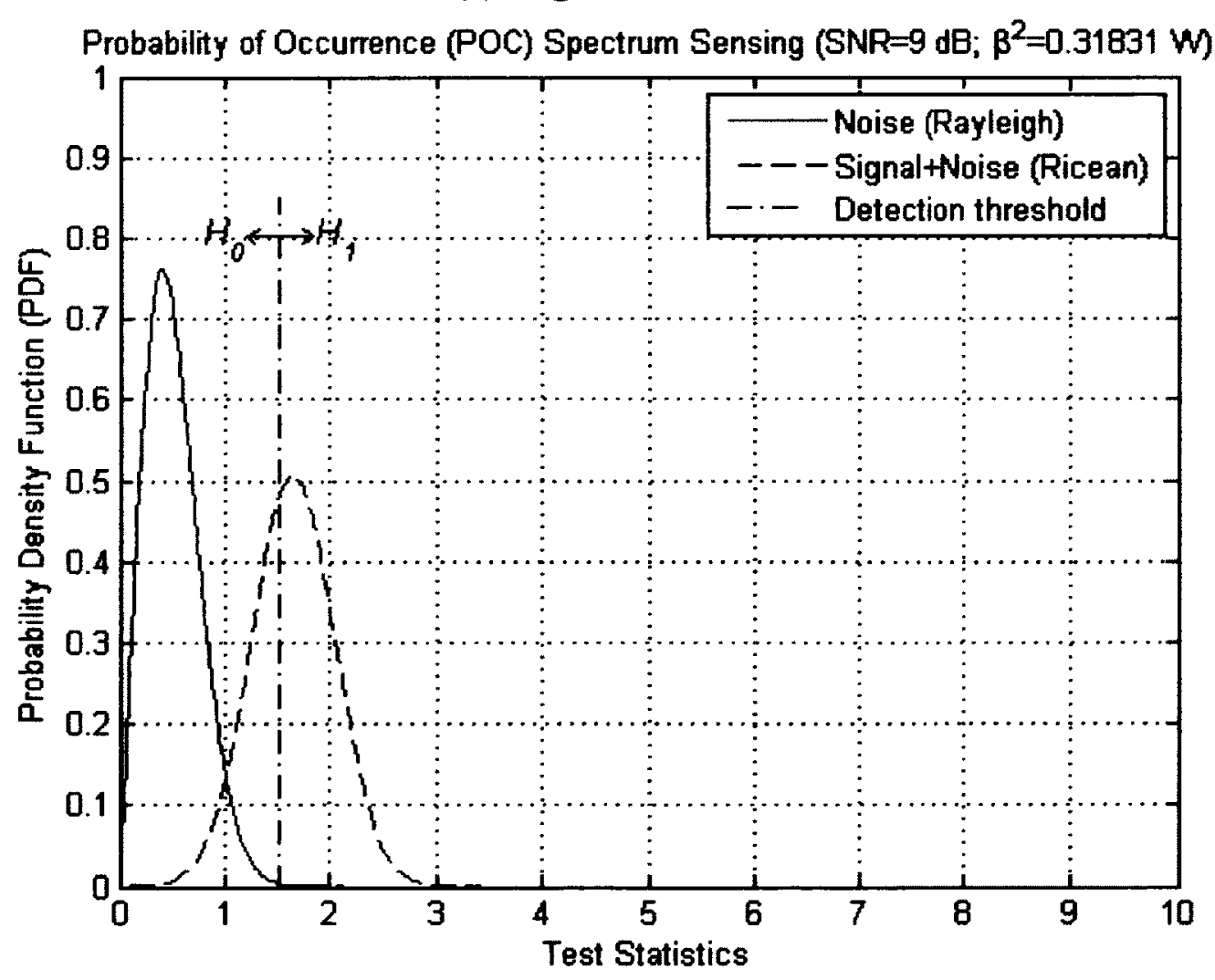

(b) Low Noise Power

Figure 5.5. Probability of Occurrence (POC) $P_{D}$ and $P_{F A}$ Tradeoff by Decreasing the Noise Power 


\subsection{LTE-OFDM Experimental Waveform Results}

This section describes how the power spectrum density (PDF) are computed graphically from the experimentally generated and captured data LTE I/Q waveforms described in Section 5.2.

First, we use the Matlab function nextpower2(), which returns the smallest power of two that is greater than or equal to the absolute value of $A$. (That is, $p$ satisfies $2^{P}>=a b s(A)$ ). This function is useful for optimizing FFT operations, which are most efficient when the sequence length is an exact power of two. If $\mathbf{A}$ is non-scalar, nextpow2() returns the smallest power of two greater than or equal to length(A). For instance, for any integer $\mathbf{n}$ in the range from 513 to 1024 , nextpow $2(\mathrm{n})$ is 10 . For a 1by-30 vector $A$, length $(A)$ is 30 and nextpow $2(A)$ is 5 . Next, the $N$-point $f f t()$ sequence is computed. Then, the power spectrum density is found by taking the absolute value of the squared $f f t()$ divided by the sequence length $\mathrm{N}$ and the sampling frequency Fs. Finally, a shift zero-frequency component is performed to center of the spectrum to the origin by using the matlab function fftshift().

Figures 5.6 to 5.11 show the power density spectrums (PSDs) for 1.4, 3, 5, 10, 15, and 20MHz scalable bandwidth of LTE-OFDM signals. The theoretical and measured bandwidths as well as the power spectral density are summarized in the table below.

\begin{tabular}{|c|c|c|}
\hline Bandwidth (Theory) & Bandwidth (Measured) & Power Spectral Density (PSD) \\
\hline $1.4 \mathrm{MHz}$ & $1.3 \mathrm{MHz}$ & $-30 \mathrm{~dB} / \mathrm{Hz}$ to $15 \mathrm{~dB} / \mathrm{Hz}$ \\
\hline $3 \mathrm{MHz}$ & $2.8 \mathrm{MHz}$ & $-40 \mathrm{~dB} / \mathrm{Hz}$ to $9 \mathrm{~dB} / \mathrm{Hz}$ \\
\hline $5 \mathrm{MHz}$ & $4.5 \mathrm{MHz}$ & $-50 \mathrm{~dB} / \mathrm{Hz}$ to $9 \mathrm{~dB} / \mathrm{Hz}$ \\
\hline $10 \mathrm{MHz}$ & $9.6 \mathrm{MHz}$ & $-60 \mathrm{~dB} / \mathrm{Hz}$ to $5 \mathrm{~dB} / \mathrm{Hz}$ \\
\hline $15 \mathrm{MHz}$ & $14.2 \mathrm{MHz}$ & $-60 \mathrm{~dB} / \mathrm{Hz}$ to $5 \mathrm{~dB} / \mathrm{Hz}$ \\
\hline $20 \mathrm{MHz}$ & $18.4 \mathrm{MHz}$ & $-60 \mathrm{~dB} / \mathrm{Hz}$ to $5 \mathrm{~dB} / \mathrm{Hz}$ \\
\hline
\end{tabular}

Table 4. LTE-OFDM Experimental I/Q Waveforms Measured Bandwidth and PSD 


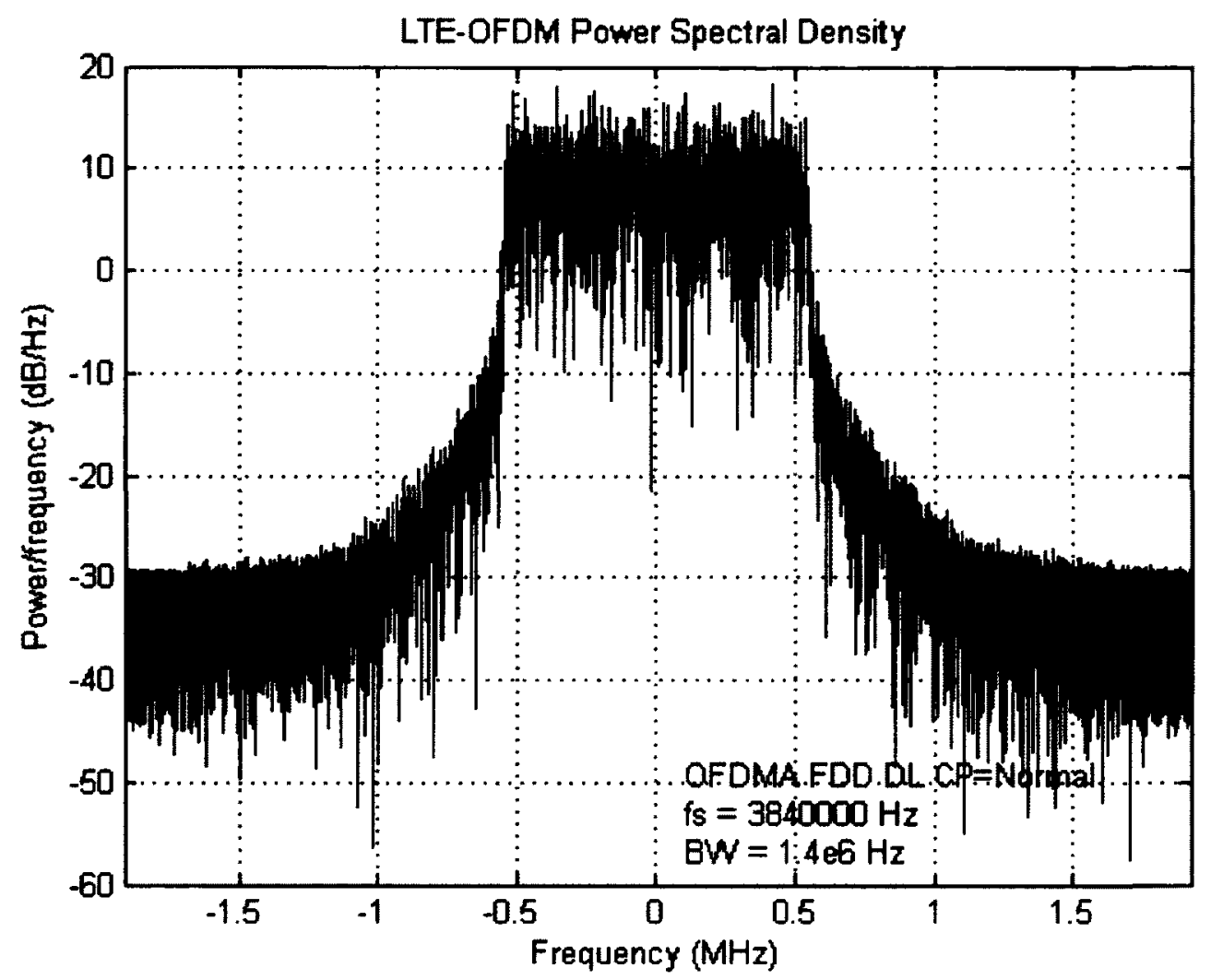

Figure 5.6. LTE-OFDM DL BW=1.4MHz Power Spectrum Density (PSD)

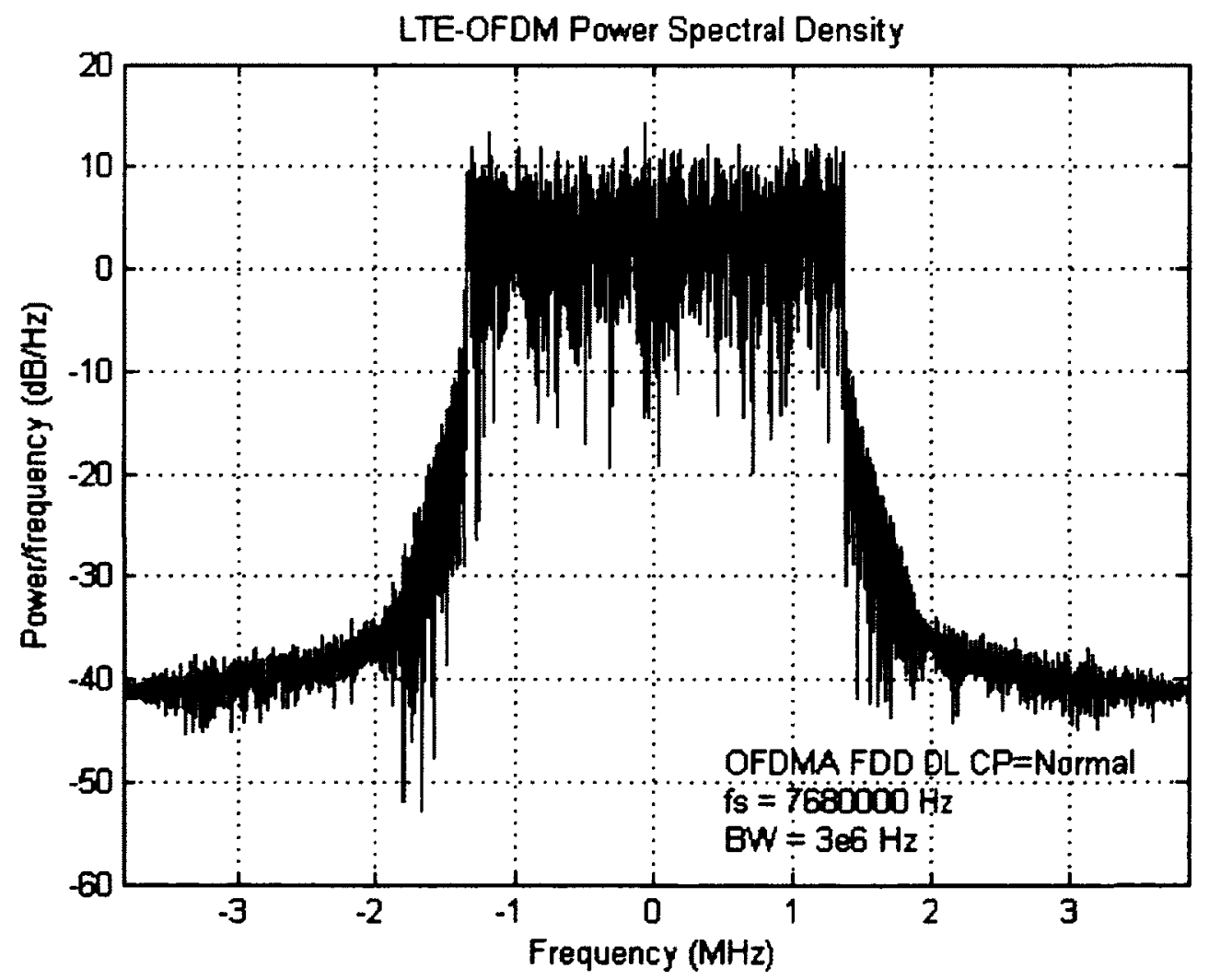

Figure 5.7. LTE-OFDM DL BW=3MHz Power Spectrum Density (PSD) 


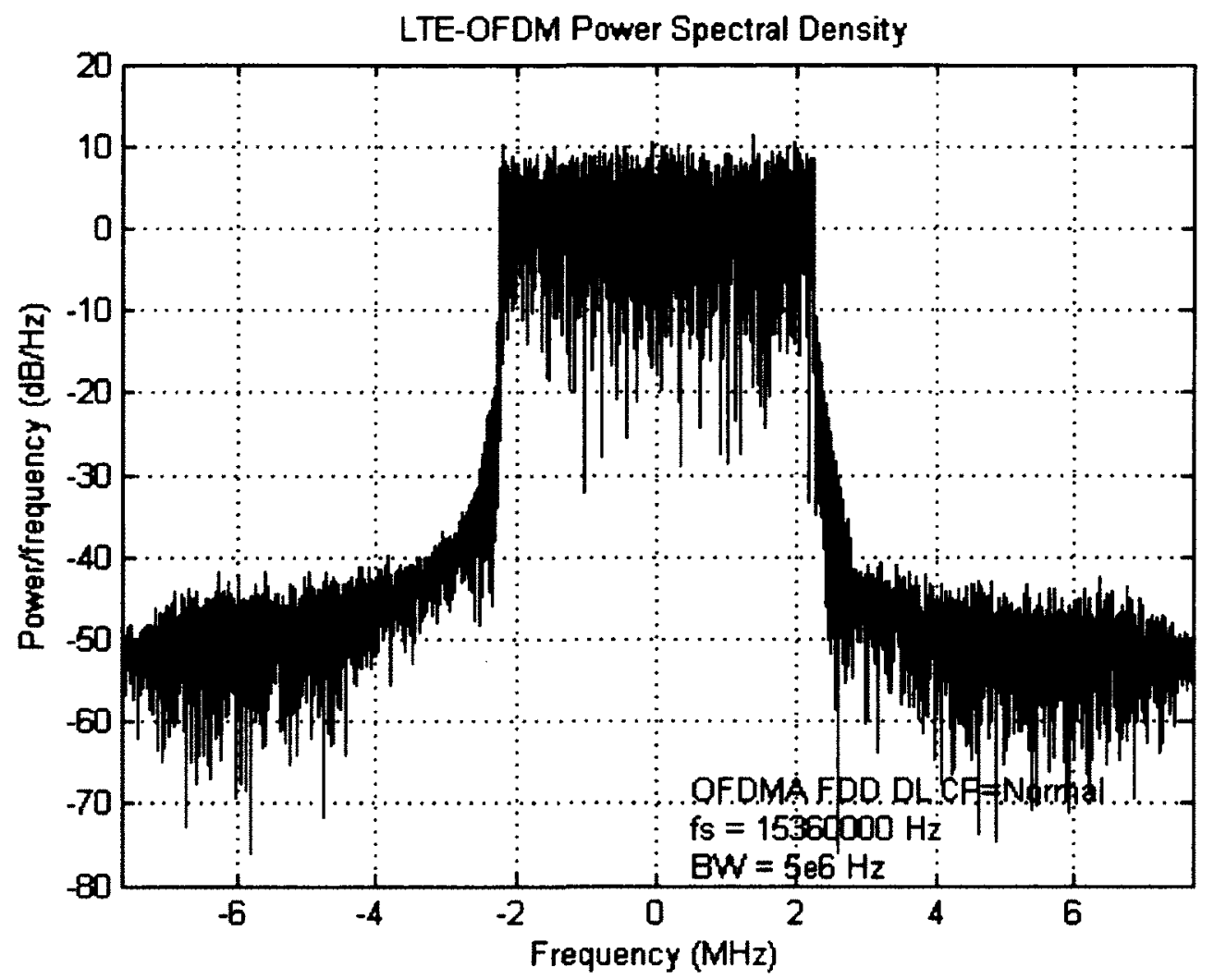

Figure 5.8. LTE-OFDM DL BW=5MHz Power Spectrum Density (PSD)

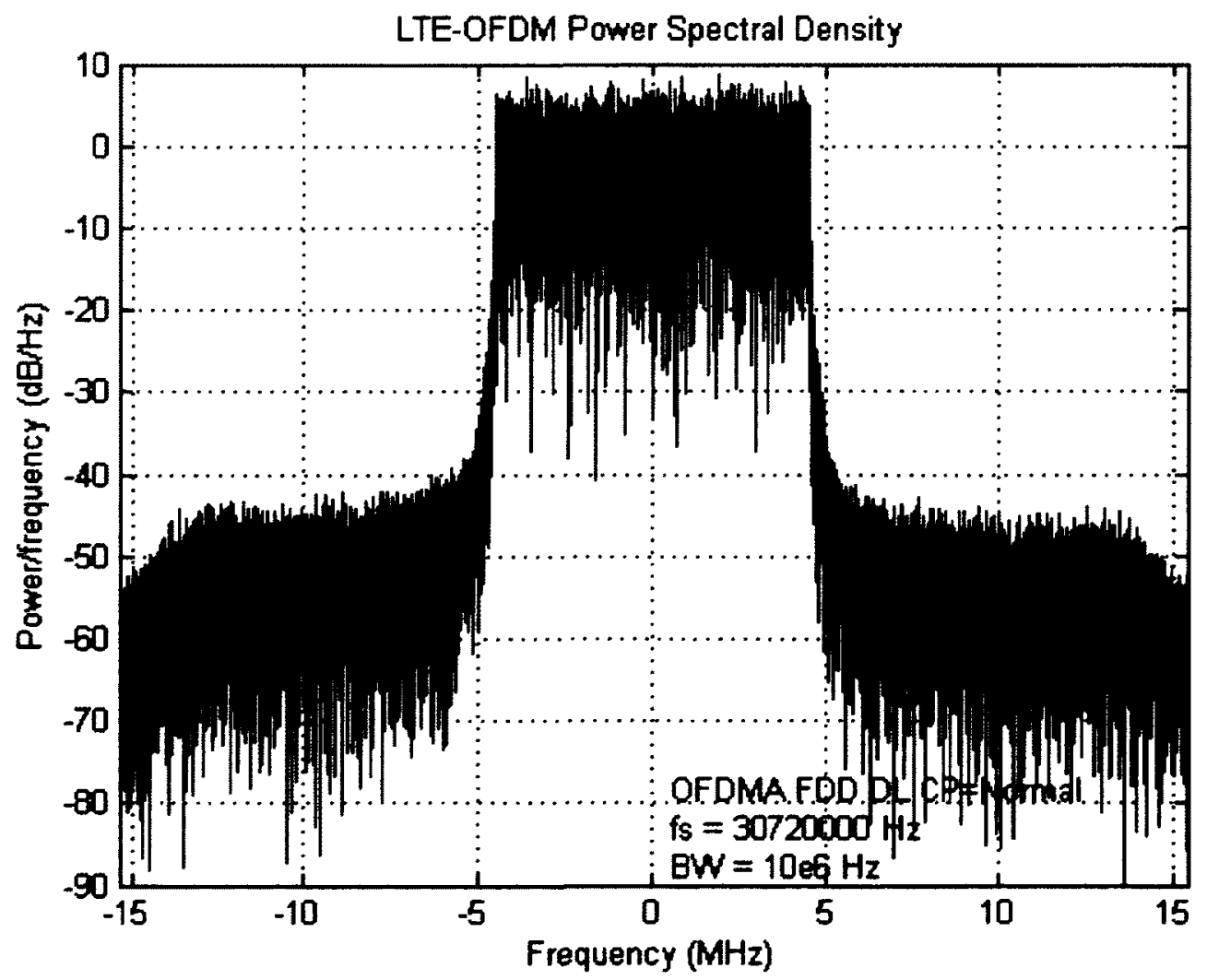

Figure 5.9. LTE-OFDM DL BW=10MHz Power Spectrum Density (PSD) 


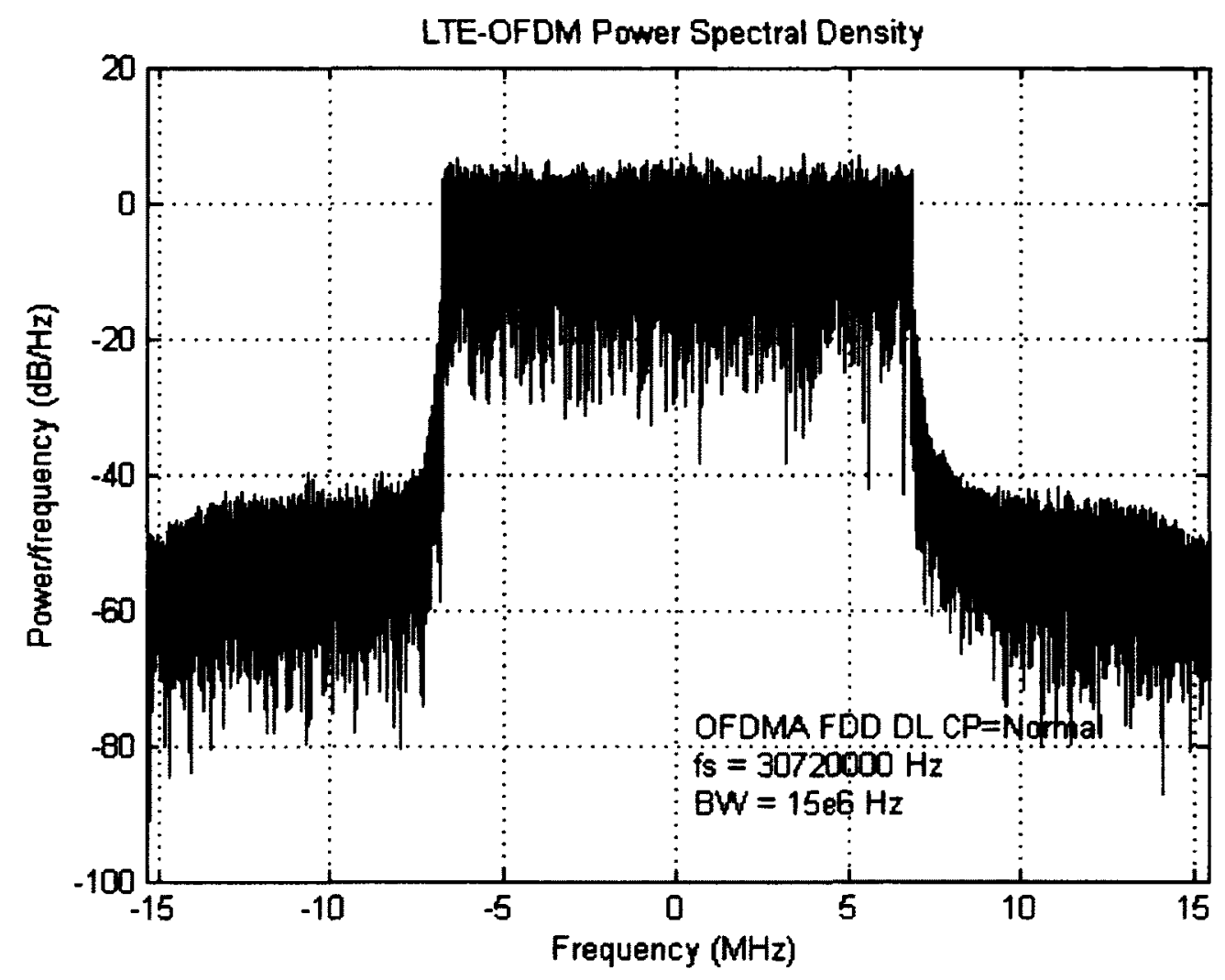

Figure 5.10. LTE-OFDM DL BW=15MHz Power Spectrum Density (PSD)

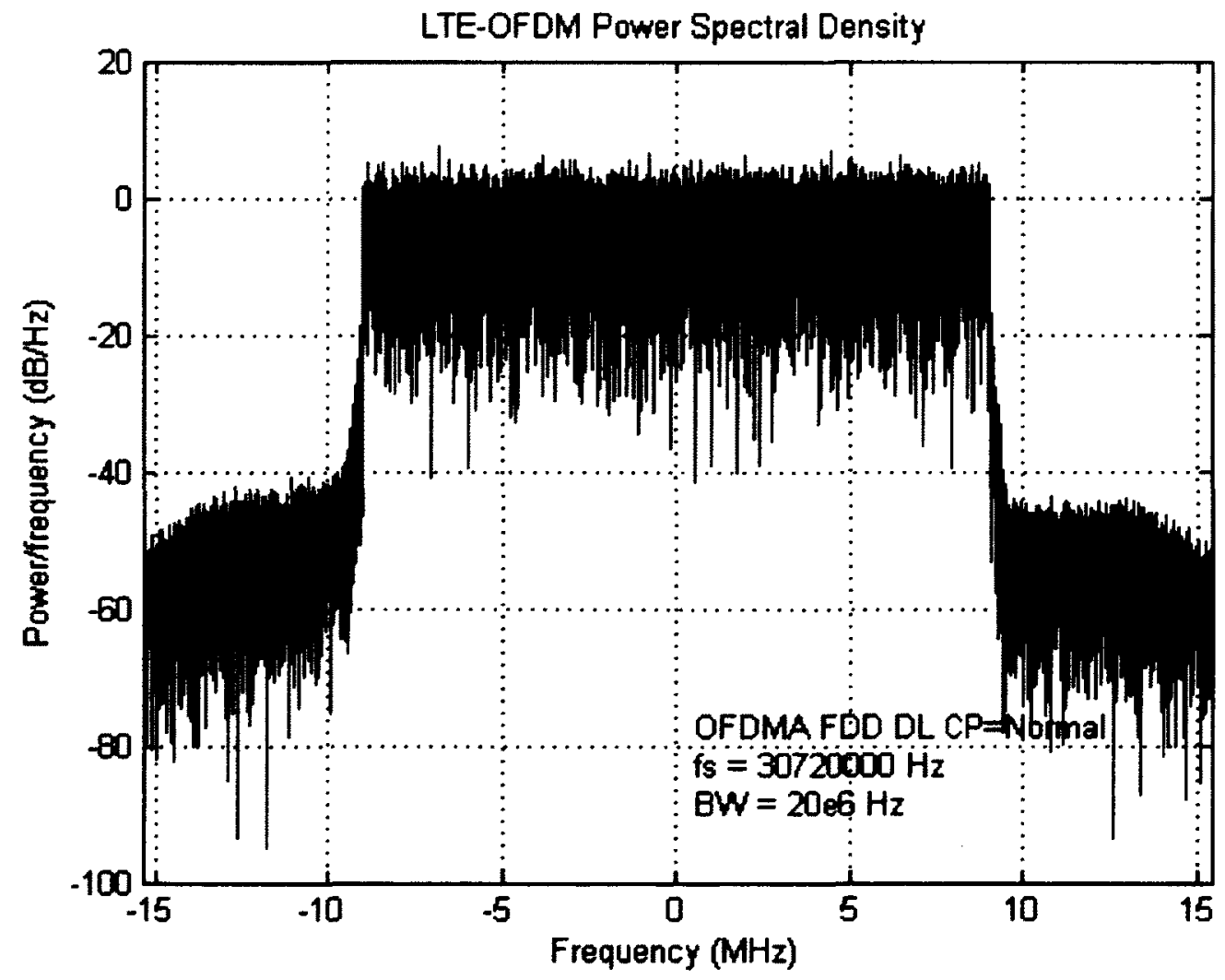

Figure 5.11. LTE-OFDM DL BW=20MHz Power Spectrum Density (PSD) 


\subsection{BER/SER vs. SNR Performance Results}

This section describes how the bit error rate (BER) and symbol error rate (SER) performance versus signal to noise ratio (SNR) for 4-QAM, 16-QAM, 64-QAM, 128-QAM, and 256-QAM modulation schemes are simulated for LTE / LTE-Advanced waveforms coherently demodulated over AWGN, Rayleigh, and Ricean channels.

Figures 5.12 to 5.19 show the BER (bit-error rate) and SER (symbol-error rate) performance versus Eb/N0 over AWGN, Rayleigh, and Ricean channels for 2-QAM, 4-QAM, 16-QAM, and 64-QAM digital modulation schemes supported in LTE and LTE-Advanced. Higher modulation order schemes are also given since these are being considered for 4G LTE-Advanced: 128-QAM (8x8 MIMO) and 256-QAM.

In order to compute the BER/SER vs. SNR over AWGN channel in Figure 5.12 and 5.13 , we use the Matlab function berawgn() which is the bit error rate (BER) for uncoded AWGN channels. $[B E R, S E R]=$ berawgn (EbNo, ' $q a m$ ', $M$ ) returns the BER of uncoded QAM over an AWGN channel with coherent demodulation. The alphabet size, $M$, must be at least 4 . When $k=\log _{2}(M)$ is odd, a rectangular constellation of size $M=I X J$ is used, where $I=2^{(k-1) / 2}$ and $J=2^{(k+1) / 2}$. The berawgn() function returns the BER of various modulation schemes over an additive white Gaussian noise (AWGN) channel. The first input argument, $E_{b} / N_{0}$, is the ratio of bit energy to noise power spectral density, in $\mathrm{dB}$. If $E_{b} / N_{0}$ is a vector, the output $B E R$ is a vector of the same size, whose elements correspond to the different $E_{b} / N_{0}$ levels. The supported modulation scheme, which correspond to the second input argument to the function for LTE and LTE-Advanced is the quadrature amplitude modulation (QAM).

Most LTE modulation schemes syntaxes also have an $M$ input that specifies the alphabet size for the modulation. $M$ must have the form $2^{k}$ for some positive integer $k$. For all cases, the berawgn function assumes the use of a Gray-coded signal constellation. If we use binary coding, the results may differ. 


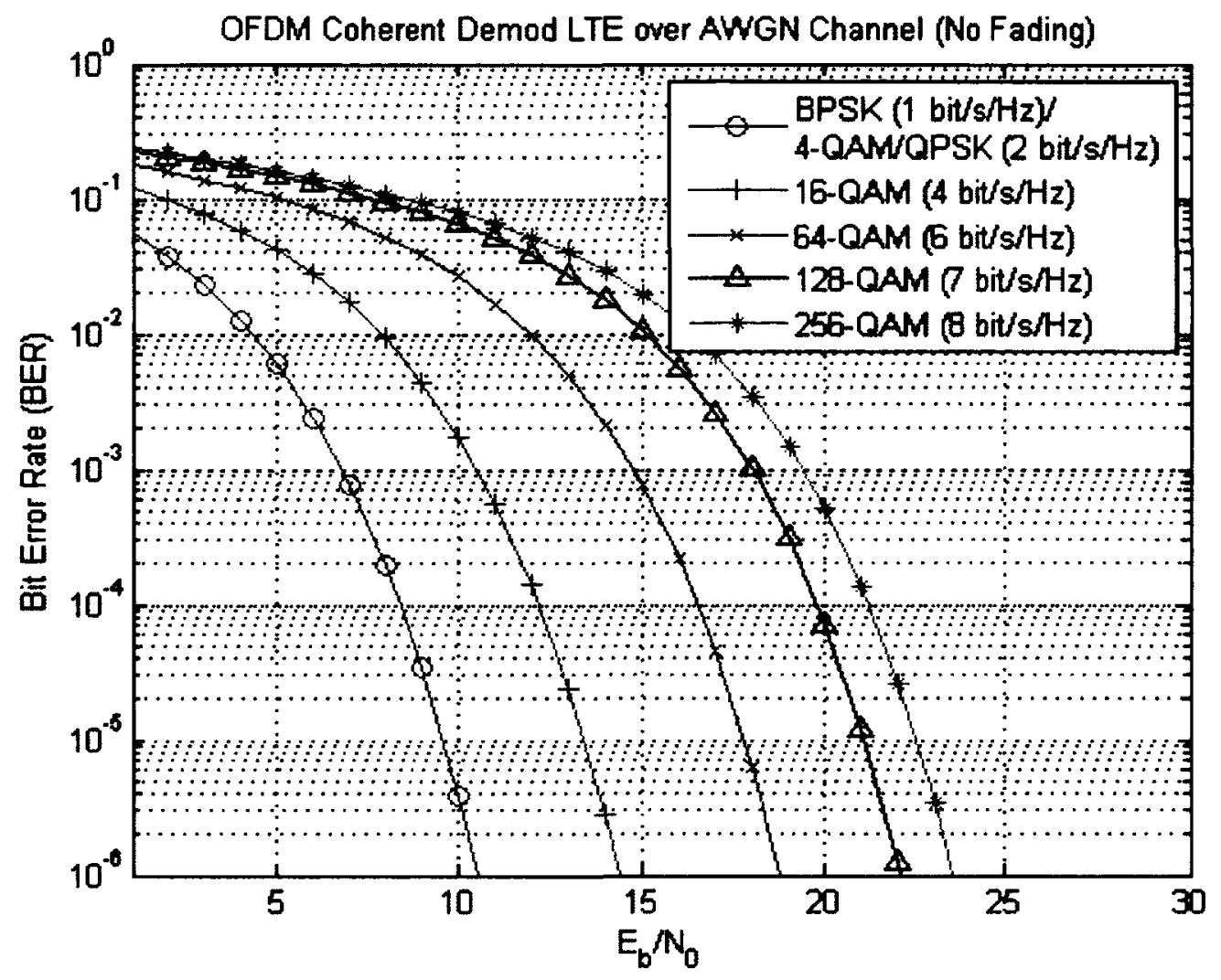

Figure 5.12. Theoretical BER OFDM Coherent Demod LTE/LTE-A over AWGN Channel

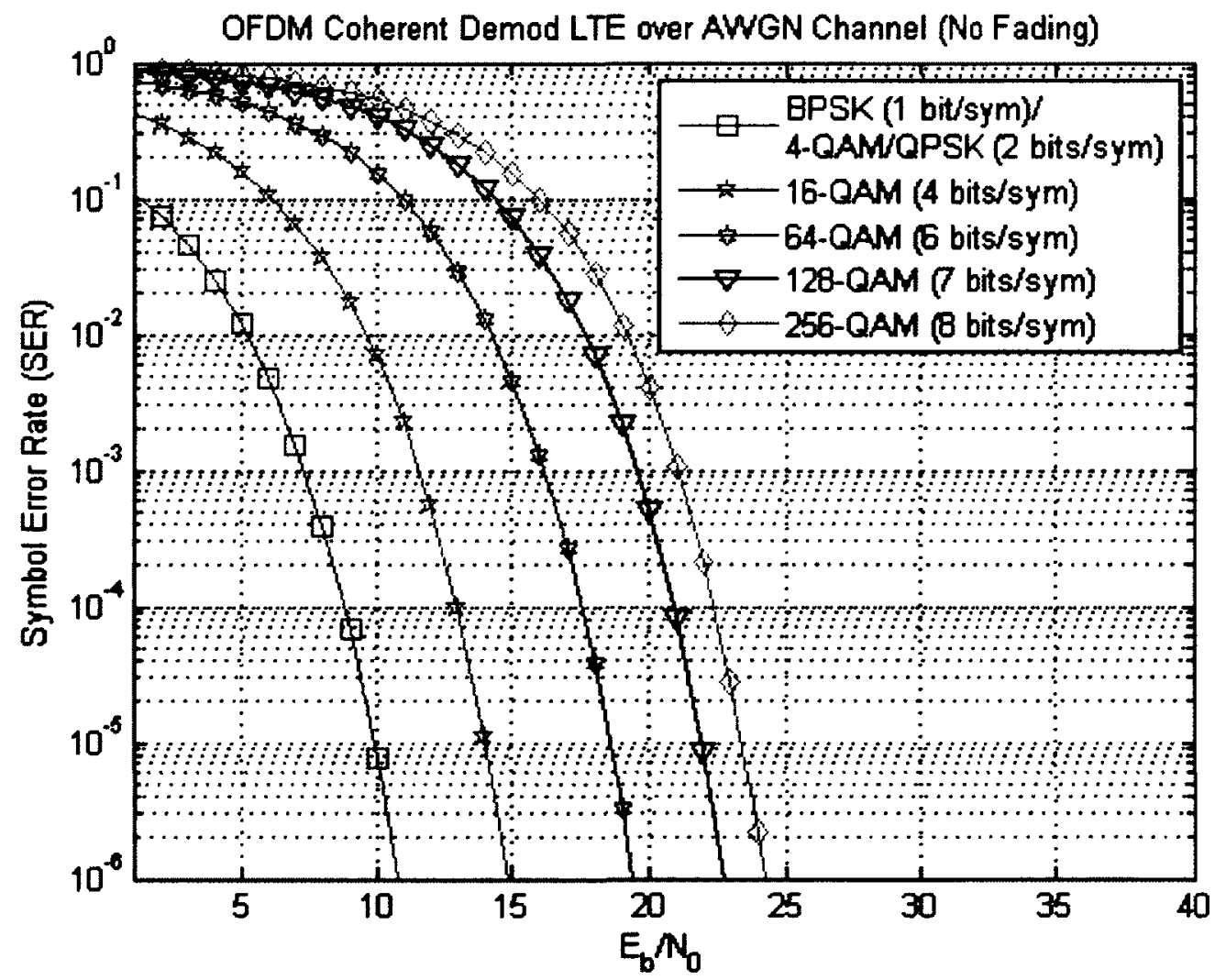

Figure 5.13. Theoretical SER OFDM Coherent Demod LTE/LTE-A over AWGN Channel 


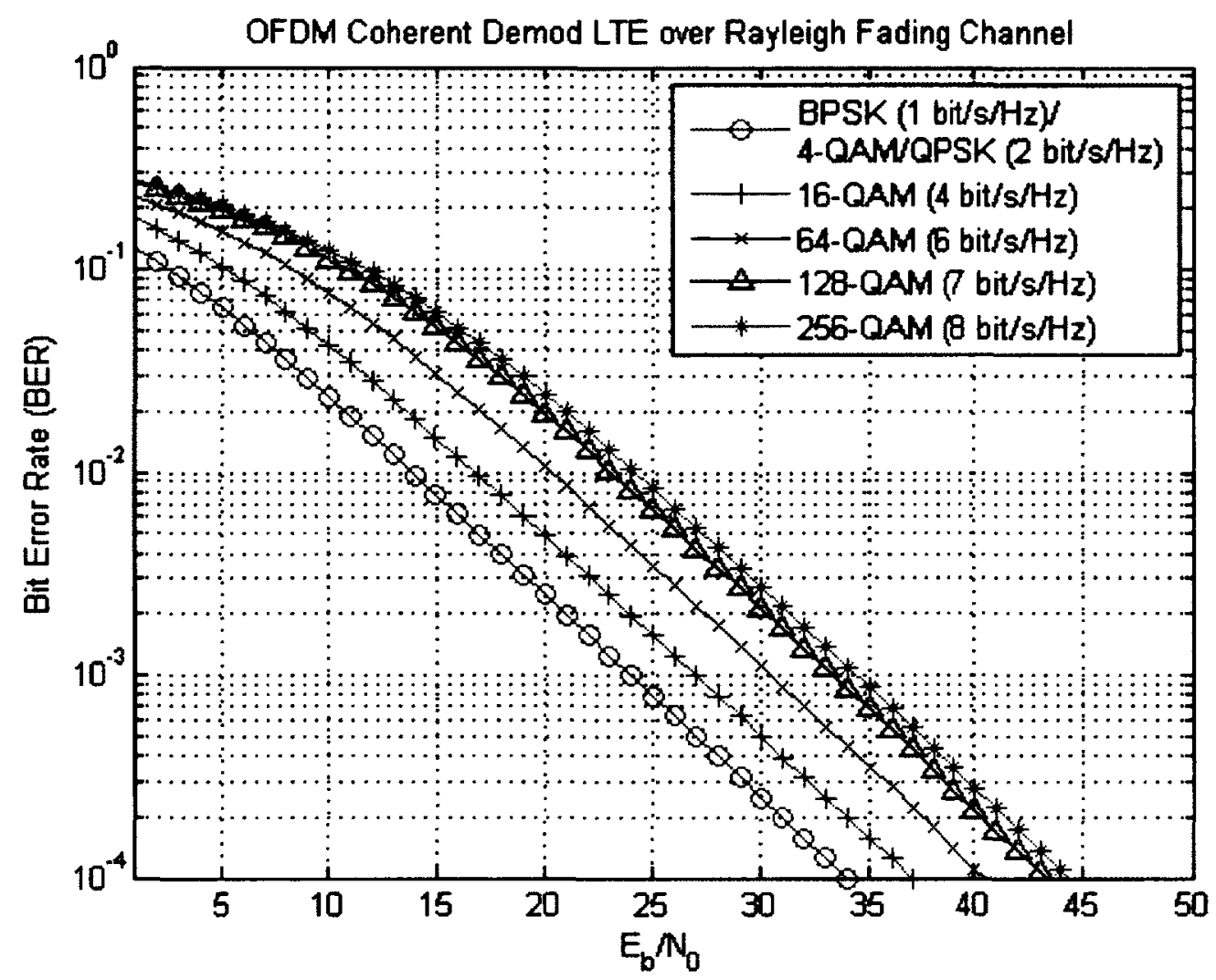

Figure 5.14. Theoretical BER Coherent Demod LTE over Rayleigh Fading Chan

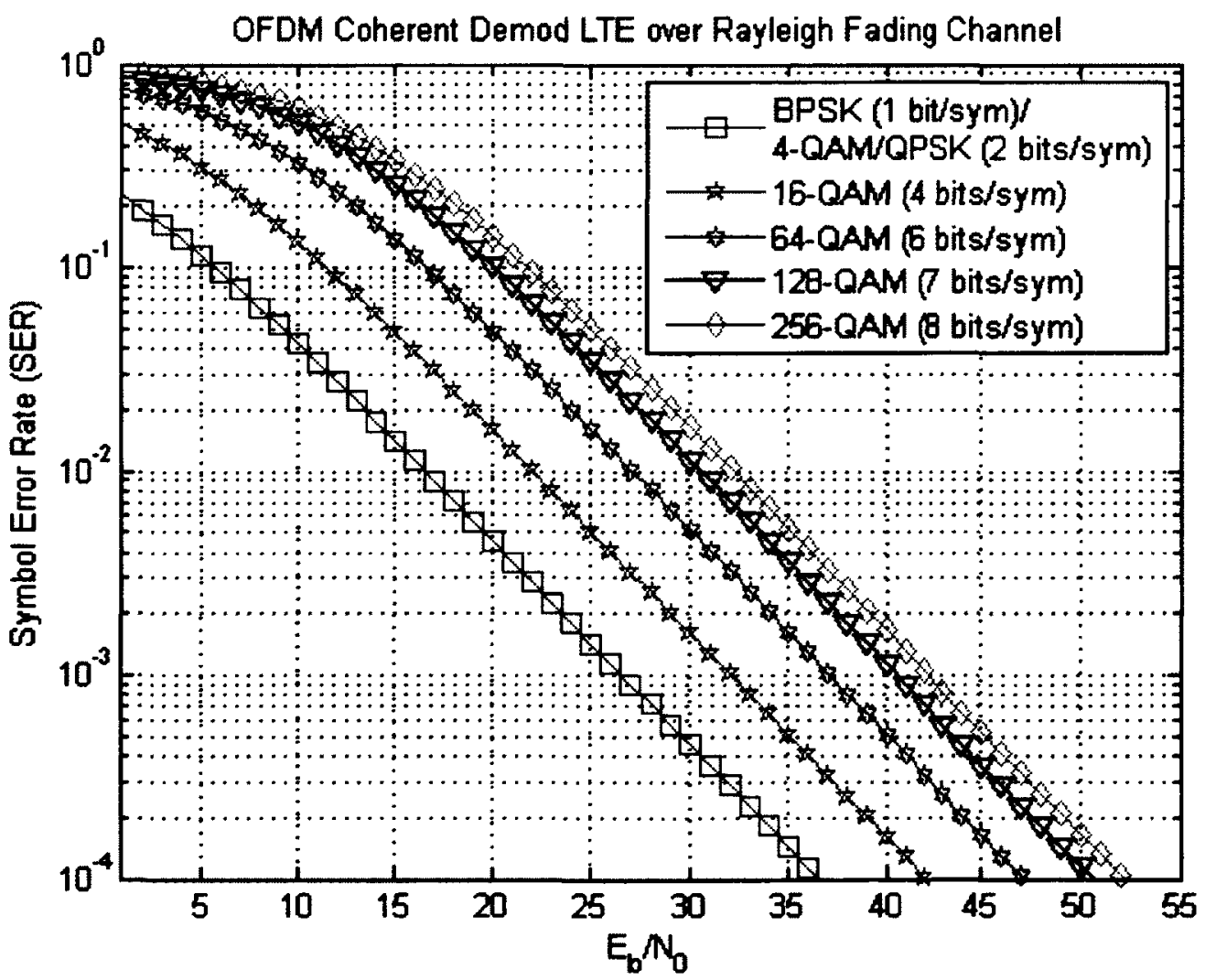

Figure 5.15. Theoretical SER Coherent Demod LTE over Rayleigh Fading Chan 
In Figures 5.14 to 5.15 , in order to compute the BER/SER vs. SNR over fading channels (Rayleigh fading) and Figures 5.16 to 5.19 (Ricean fading), we use the Matlab function berfading () which returns the bit error rate (BER) for Rayleigh and Rician fading channels. [BER, SER] = berfading (EbNo, ' qam' , M, divorder, $K$ ) returns the BER for QAM over an uncoded Rayleigh fading $(K=0)$ and Ricean fading $(K>0)$ channels with coherent demodulation, where $K=\mu^{2} / \sigma^{2}$ is the ratio of specular to diffuse energy in the linear scale. The default $\mathrm{K}$ value is 1 (a line-of-sight component on the first path only).

The alphabet size, $M$, must be at least 4 . When $k=\log _{2}(M)$ is odd, a rectangular constellation of size $M=I \times J$ is used, where $I=2^{(k-1) / 2}$ and $J=2^{(k+1) / 2}$. The first input argument, $E_{b} / N_{0}$, is the ratio of bit energy to noise power spectral density, in $d B$. If $E_{b} / N_{0}$ is a vector, the output $B E R$ is a vector of the same size, whose elements correspond to the different $E_{b} / N_{0}$ levels. For cases where diversity is used, the SNR on each diversity branch is $E_{b} / N_{0} /$ divorder, where divorder is the diversity order (the number of diversity branches) and is a positive integer. It was shown in Figures 5.16 to 5.19 that as the ratio of specular to diffuse energy $K$ increases from $K=3$ to $K=6, E_{b} / N_{0}$ decreases.

As illustrated in Figures 5.12 to 5.19, for higher order QAM constellation (higher data rate and mode) used in 4G/LTE and RF/microwave QAM application environments (such as in broadcasting or telecommunications), multipath interference typically increases. There is a spreading of the spots in the constellation, decreasing the separation between adjacent states, and making it difficult for the receiver to decode the signal appropriately. That is, there is reduced noise immunity in high order QAM. By moving to a higher-order constellation, it is possible to transmit more bits per symbol. However, if the mean energy of the constellation is to remain the same, the points must be closer together and are thus more susceptible to noise and other corruption. This results in a higher bit error rate (BER) and so higher-order QAM can deliver more data less reliably and typically requires a higher $E_{b} / N_{0}$ than lower-order QAM. 


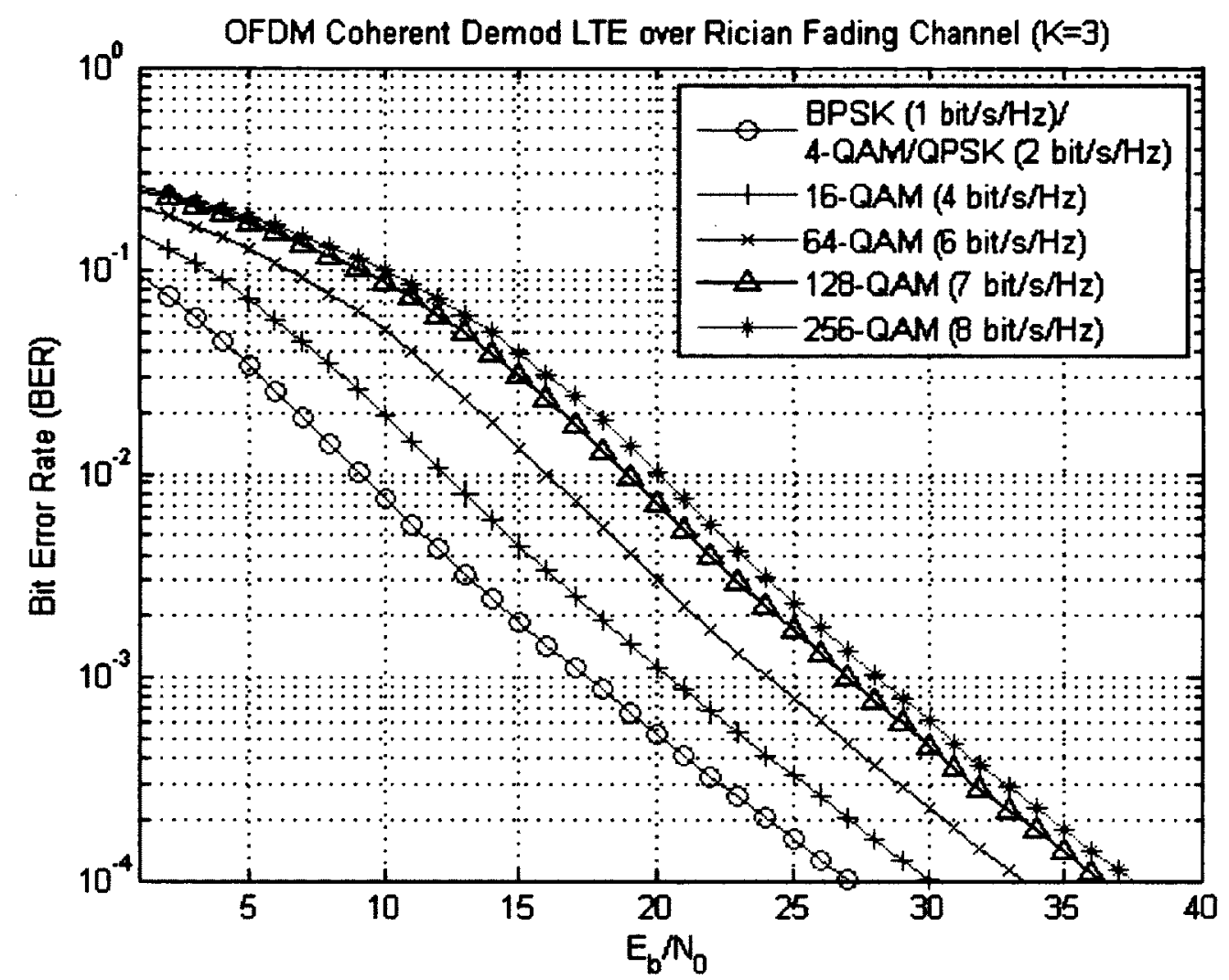

Figure 5.16. Theoretical BER Coherent Demod LTE over Rician Fading Chan $(K=3)$

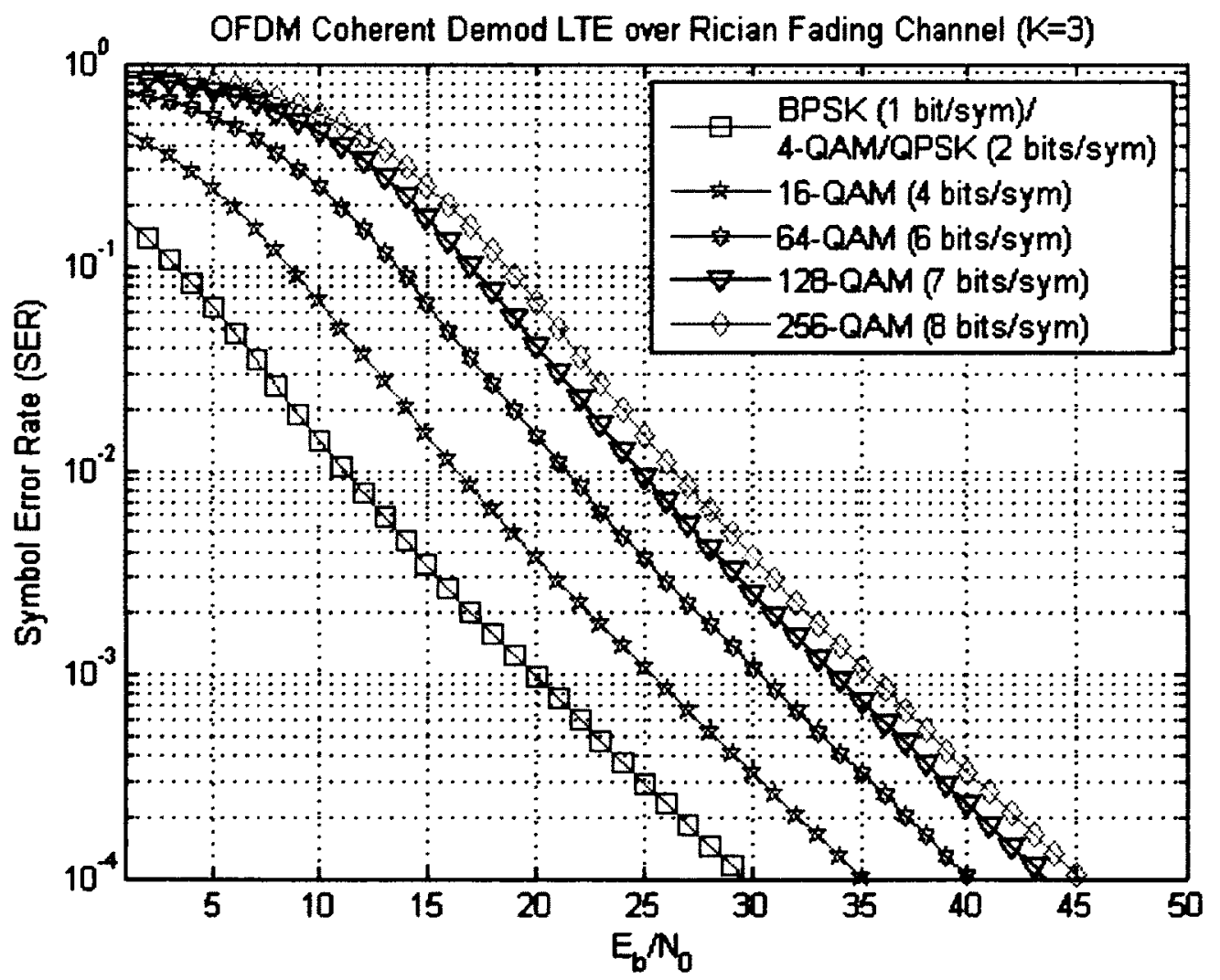

Figure 5.17. Theoretical SER Coherent Demod LTE over Rician Fading Chan $(K=3)$ 


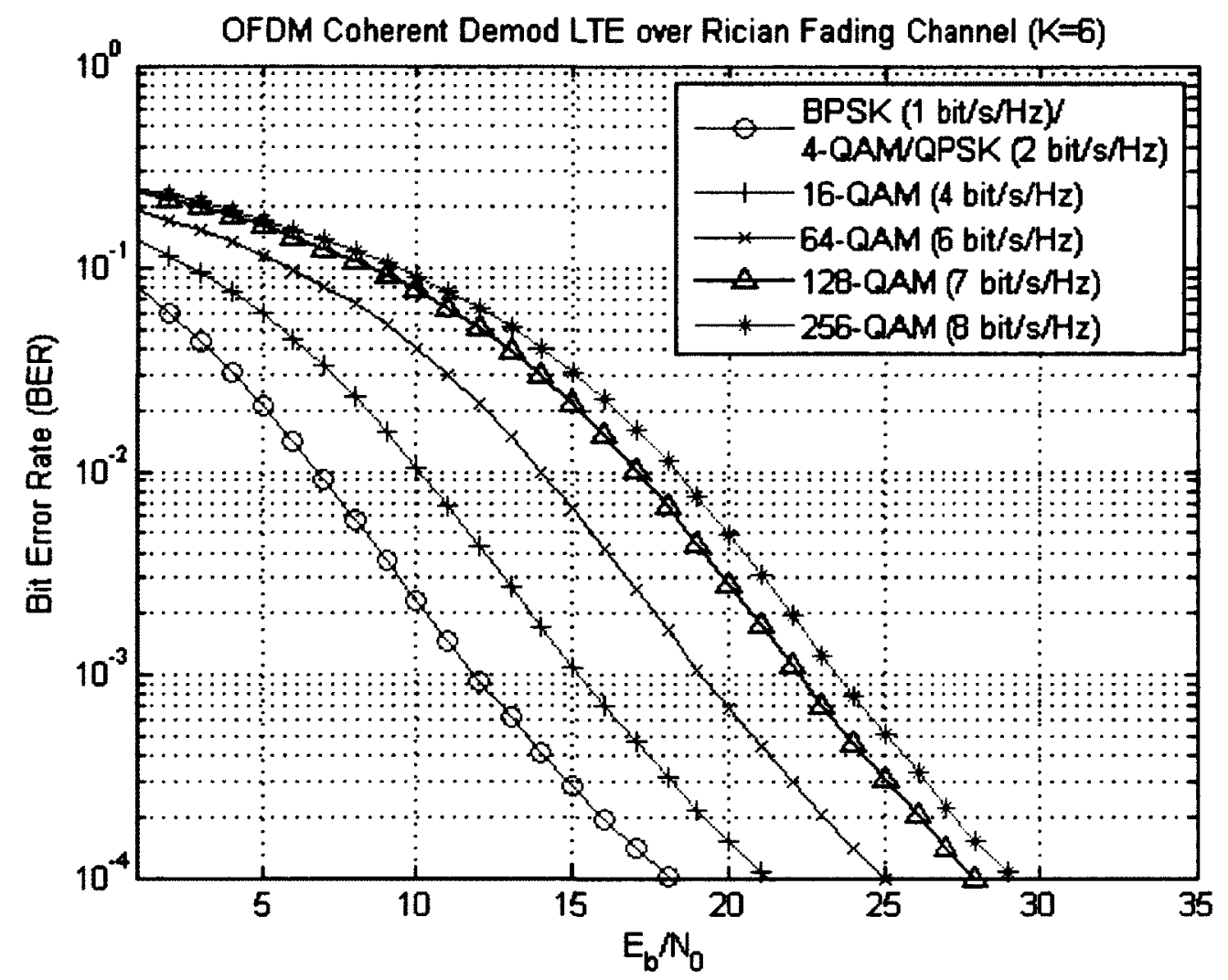

Figure 5.18. Theoretical BER Coherent Demod LTE over Rician Fading Chan $(K=6)$

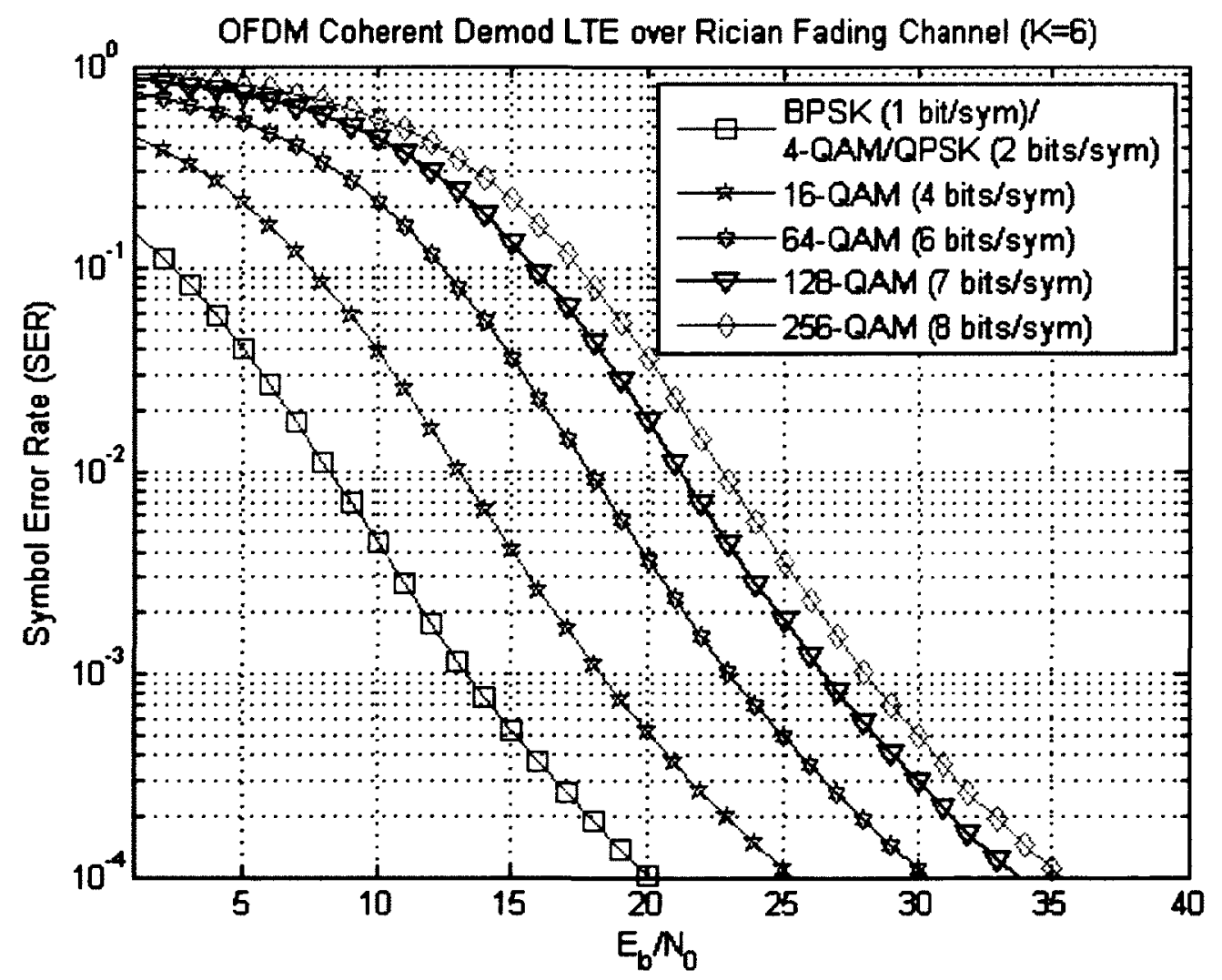

Figure 5.19. Theoretical SER Coherent Demod LTE over Rician Fading Chan $(K=6)$ 


\subsection{Symbol Timing Offet Estimation Experimental Results}

This section describes the sliding window algorithm experimental implementation in Matlab for the symbol timing offset estimation based on LTE I/Q waveforms. The LTE waveforms have been configured by Aeroflex IQCreator Arbitrary Waveform Generator (ARB) software, and generated by Aeroflex IFR $3414(250 \mathrm{kHz}$ to $4 \mathrm{GHz})$ signal generator hardware. Symbol synchronization refers to the task of finding the precise moment an individual OFDM symbol starts and end, i.e., the correct position of the DFT window. The symbol timing result defines the FFT window, i.e. the set of samples used to calculate FFT of each received OFDM symbol. The goal in symbol timing offset (STO) estimation is to find the correct timing of a frame, a place to start the $N$-point FFT for demodulating an OFDM symbol.

The main idea of the sliding window algorithm is based on the calculation of correlation metrics that exploit some periodicity of the OFDM symbol that is due to the cyclic prefix (CP). That is, the cyclic prefix is the prepended replica of the data portion in the OFDM symbol. Consequently, the CP and the corresponding data part share their similarities that can be used for the symbol timing offset (STO) estimation. $N_{\mathrm{CP}}$ samples and another $N_{\mathrm{CP}}$ samples are spaced $N_{\mathrm{FFT}}$ samples apart. Now, consider two sliding windows, $W_{1}$ and $W_{2}$ which are spaced $N_{\mathrm{FFT}}$ samples apart. These windows can slide to find the similarity between the samples within $W_{1}$ and $W_{2}$. The similarity between two blocks of $N_{\mathrm{CP}}$ and $N_{\mathrm{CP}}$ samples in $W_{1}$ and $W_{2}$ is maximized when the cyclic prefix of an OFDM symbol falls into the first sliding window $W_{1}$.

At the sliding window algorithm start up, we need to initialize the two sliding window buffers sliding_w=zeros $(2, \mathrm{Nw})$, where $\mathrm{Nw}$ is the sliding window size and is defined as the product of the sampling frequency with the cyclic prefix duration, $\mathrm{Nw}=f s^{\star} \mathrm{T} c \mathrm{p}$. Then we need to initialize the correlation peak buffer Corr_Peaks $=[]$ and the STO buffer STO $=[$ ] . The sliding window algorithm starts with a main loop from $n=1$ to $N$ of all the received $\mathrm{V} / \mathrm{Q}$ waveform samples data. First, we update the sliding window 1 with $\mathrm{VQ}$ waveform samples. Then, sliding window 2 is shifted by $\mathrm{N}_{\mathrm{FFT}}$ samples with 
$(m=n-N f f t)$. Next, the sliding window 2 is updated with $I / Q$ waveform samples by discarding the negative index elements (storing only positive samples with loop index $m>0$ ). We compute the normalized correlation between sliding window 1 and sliding window 2 by dividing $\operatorname{corr}(n)=$ abs (sliding_w( $1,:) *$ sliding_w $(2,:) ')$ with norm(sliding_w $(1,:)) *$ norm (sliding_w( $2,:))$.

Now, the correlation threshold for peak detection is set. In this case, the correlation threshold for peak detection has been set to cor_thd $=0.9$. Finally, we perform a correlation peaks detection test against the correlation threshold in order to determine the symbol start times. The points at which the correlation is presumably maximized yield the STO estimates.

The STO start times can be obtained by the addition of the correlation peaks with the guard interval (cyclic prefix) length.

Figures 5.20 to 5.25 show the symbol timing offset synchronization with the correct timing of the frame, i.e., the estimated OFDM symbol start times. The distinct correlation peaks can be seen at one symbol interval $\left(N_{\mathrm{FFT}}\right)$ apart in the correlation results.

It is observed that the mainlobe of the STO correlation peak in the extended CP case is wider than in the normal CP case (or vice versa the mainlobe of the STO correlation peak in the normal CP is steeper than in the extended CP). This is due to the fact that the extended CP has a longer cyclic prefix length.

It is noted that the peak detection of local maxima or minima of a noisy signal is not simple since there may be spurious peaks around true peaks due to the noise. 


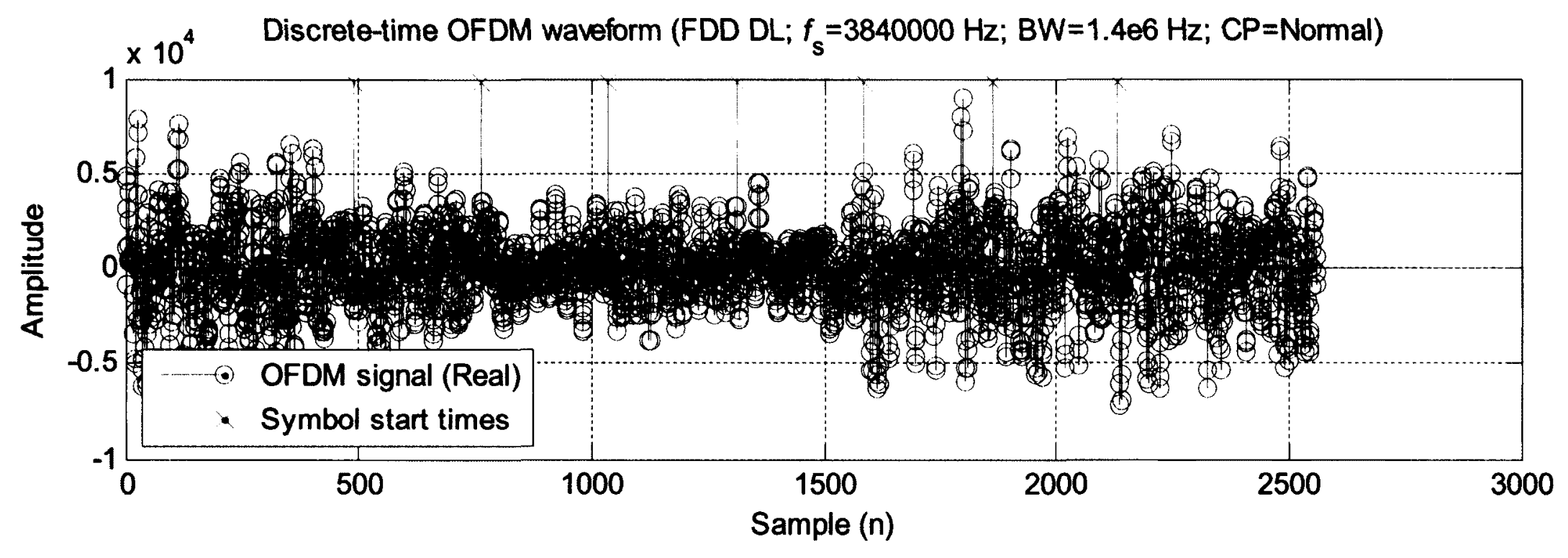

Correlation waveform between 2 sliding windows (FDD DL; $f=3840000 \mathrm{~Hz} ; \mathrm{BW}=1.4 \mathrm{e} 6 \mathrm{~Hz} ; \mathrm{CP}=\mathrm{Normal}$ )

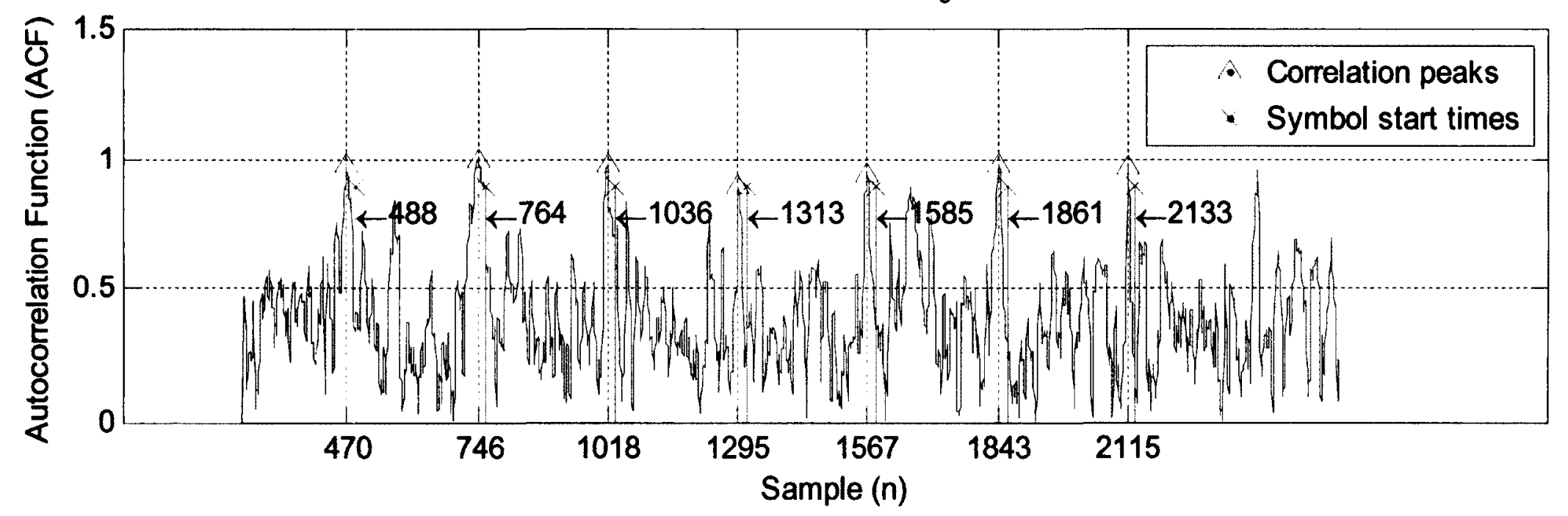

Figure 5.20. Symbol Time Offset (STO) Estimation 1.4 MHz, Downlink, CP=Normal 

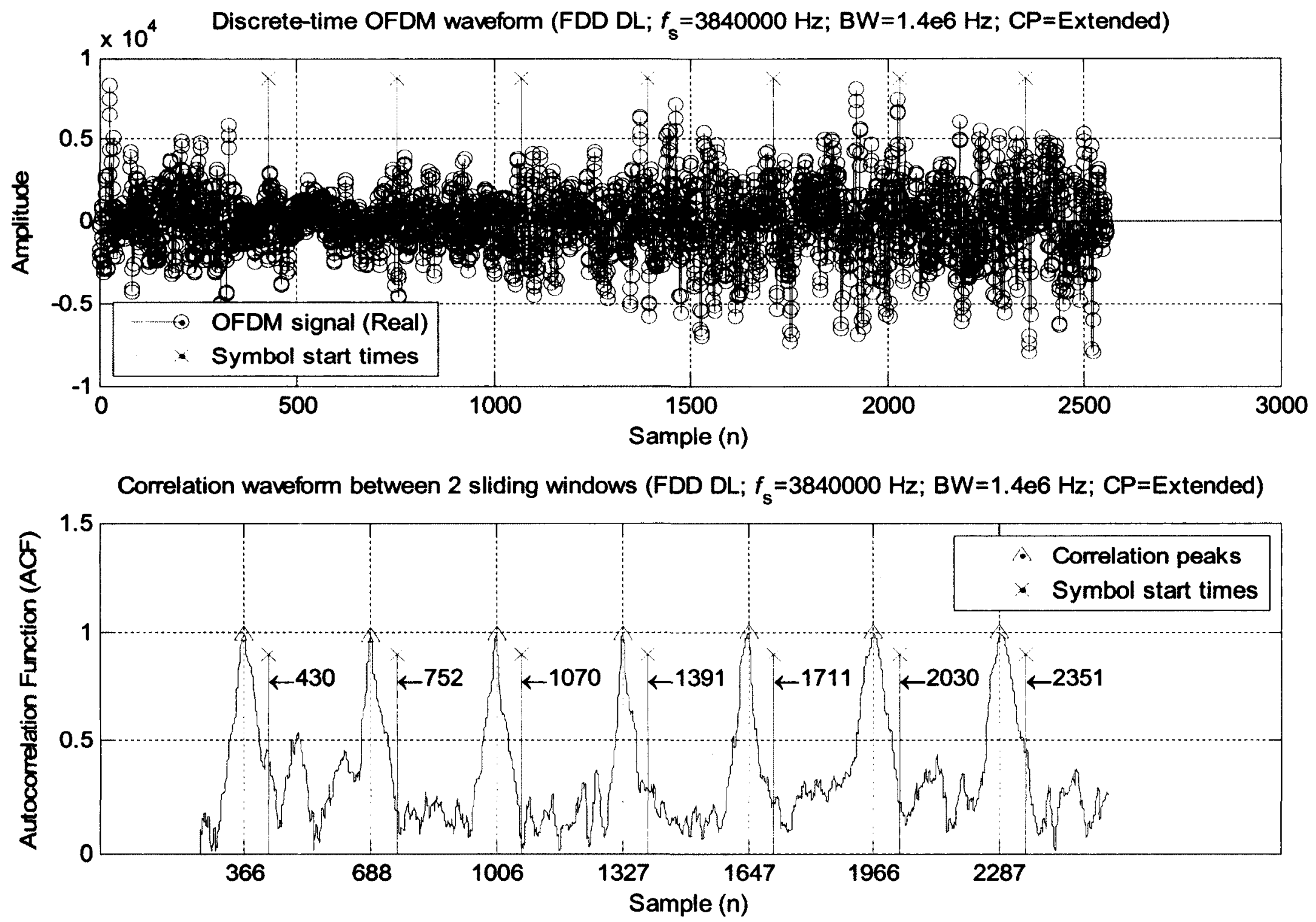

Figure 5.21. Symbol Time Offset (STO) Estimation 1.4 MHz, Downlink, CP=Extended 


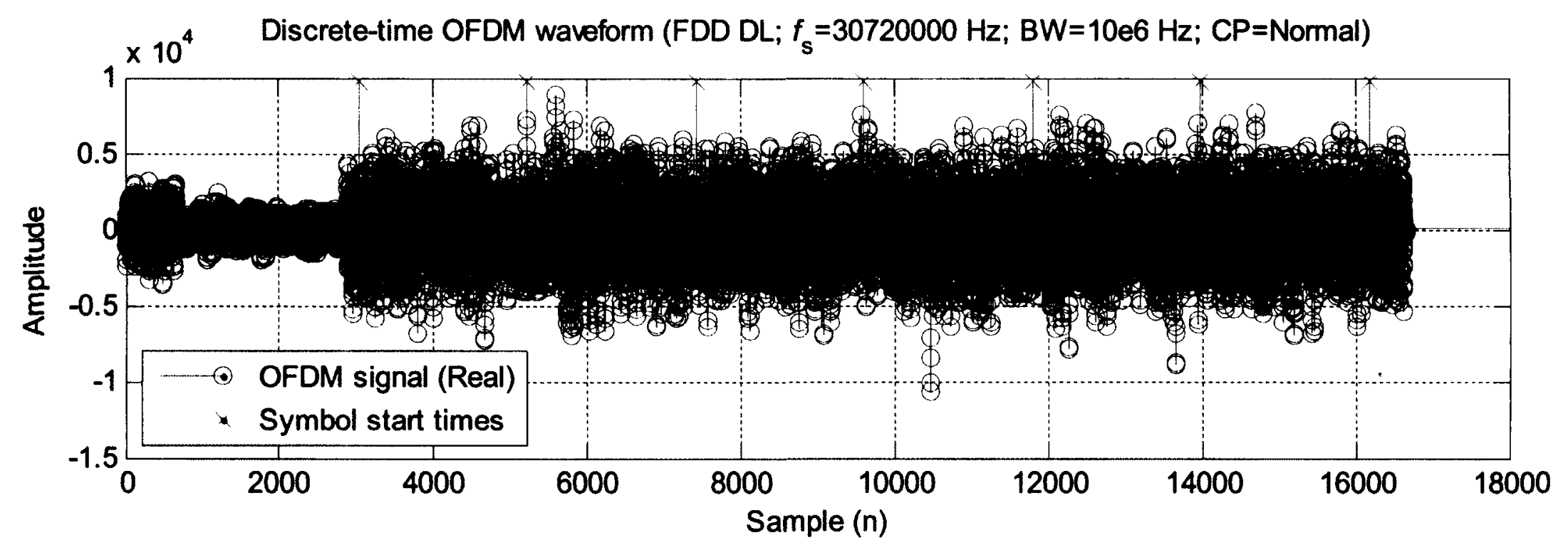

Correlation waveform between 2 sliding windows (FDD DL; $f_{\mathrm{s}}=30720000 \mathrm{~Hz} ; \mathrm{BW}=10 \mathrm{e} 6 \mathrm{~Hz} ; \mathrm{CP}=$ Normal)

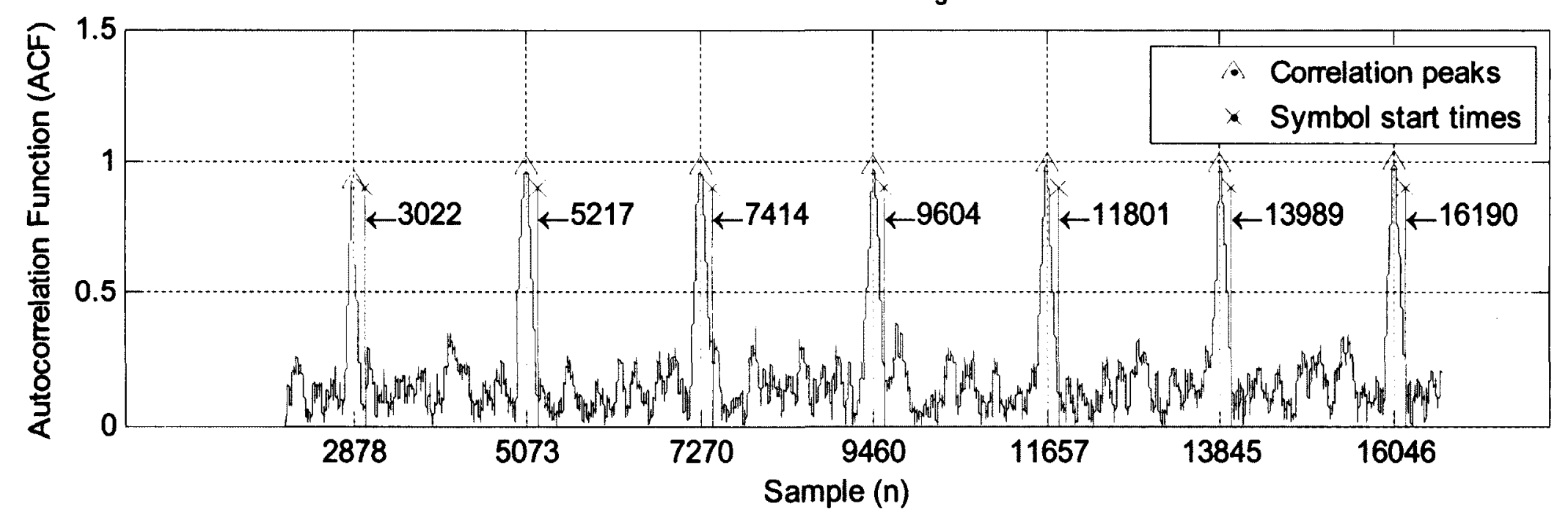

Figure 5.22. Symbol Time Offset (STO) Estimation $10 \mathrm{MHz}$, Downlink, CP=Normal 


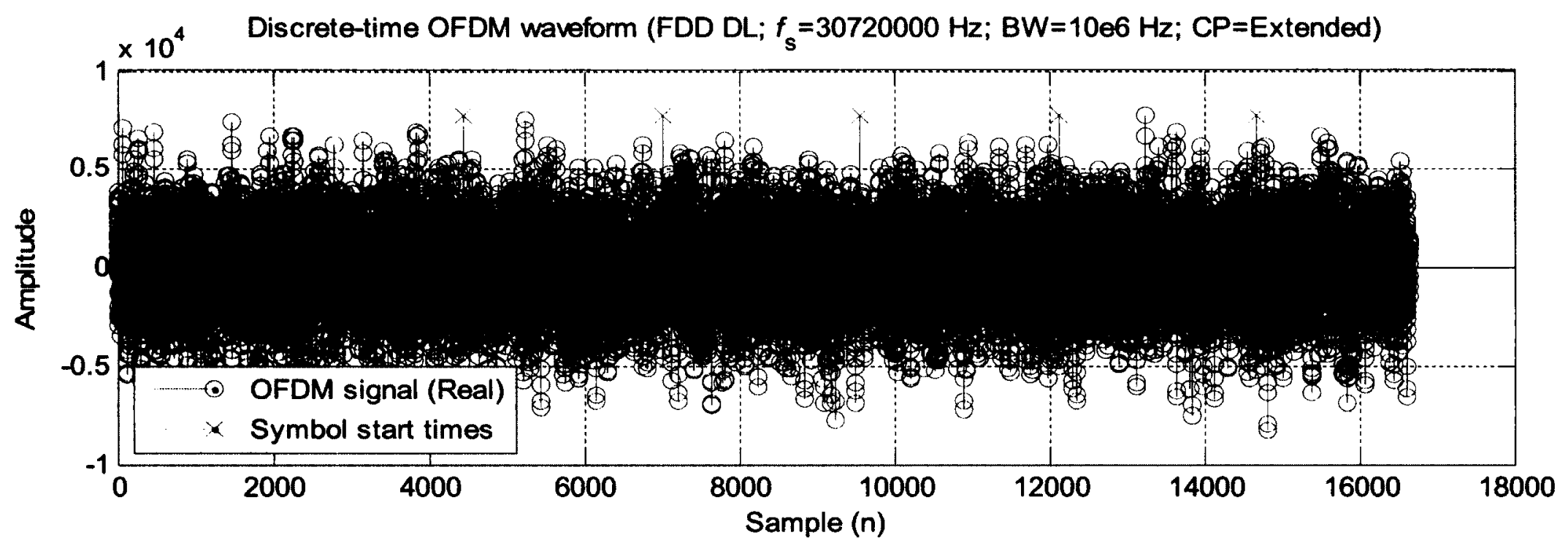

Correlation waveform between 2 sliding windows (FDD DL; $\left.f_{s}=30720000 \mathrm{~Hz} ; \mathrm{BW}=10 \mathrm{e} 6 \mathrm{~Hz} ; \mathrm{CP}=\mathrm{Extended}\right)$

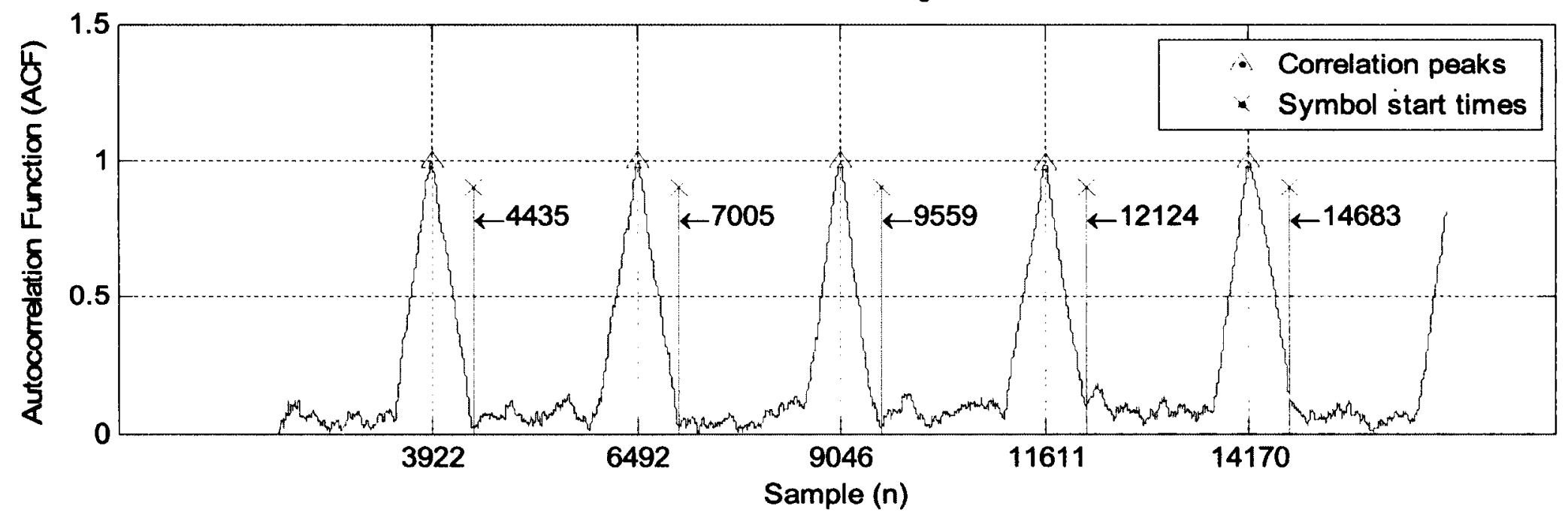

Figure 5.23. Symbol Time Offset (STO) Estimation $10 \mathrm{MHz}$, Downlink, CP=Extended 


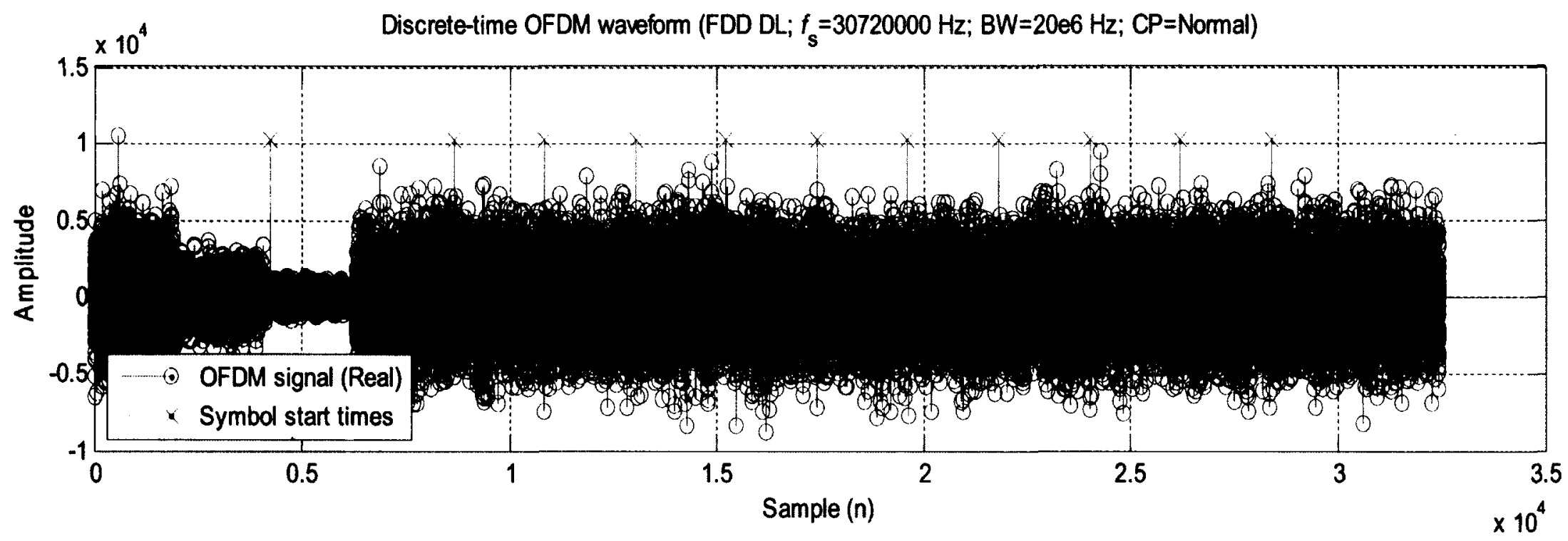

Correlation waveform between 2 sliding windows ( $F D D$ DL; $f_{s}=30720000 \mathrm{~Hz} ; B W=20 \mathrm{e} 6 \mathrm{~Hz} ; \mathrm{CP}=$ Normal)

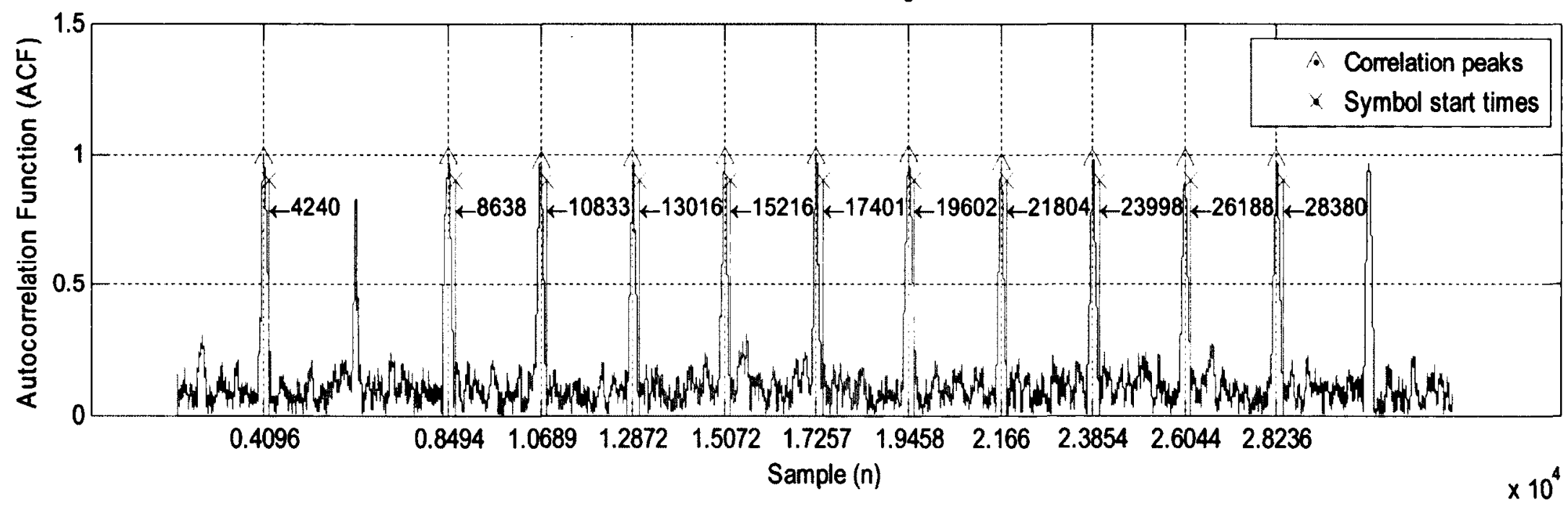

Figure 5.24. Symbol Time Offset (STO) Estimation $20 \mathrm{MHz}$ Downlink CP=Normal 


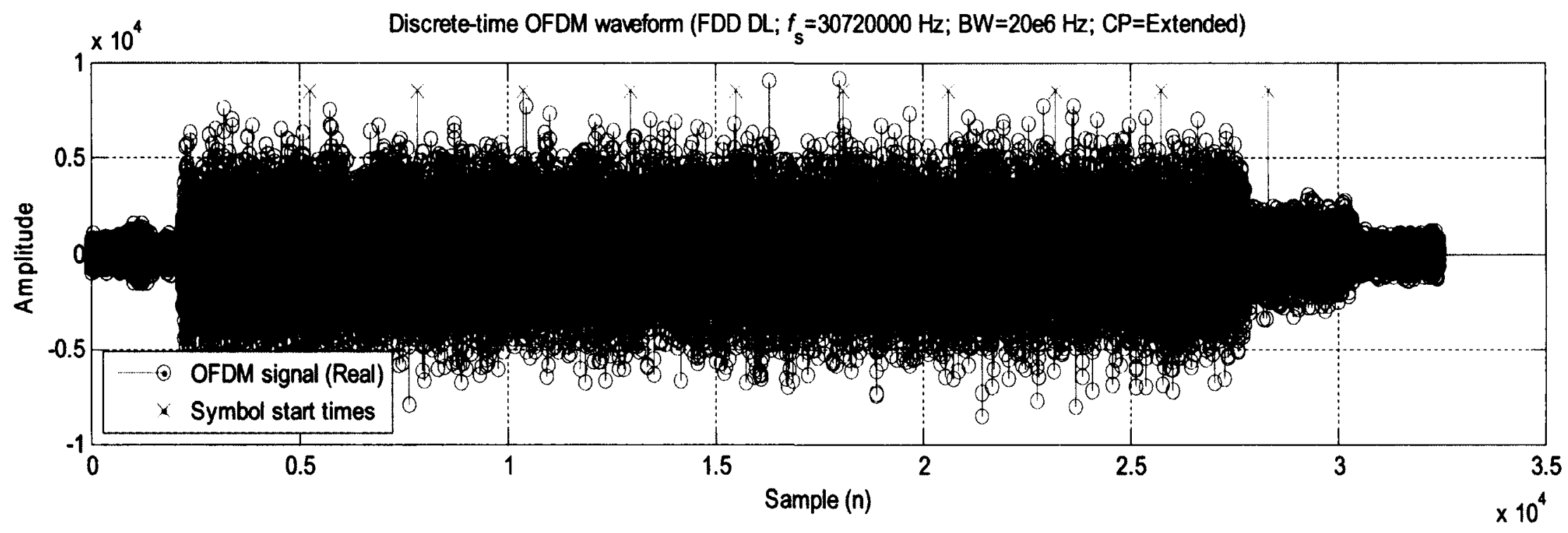

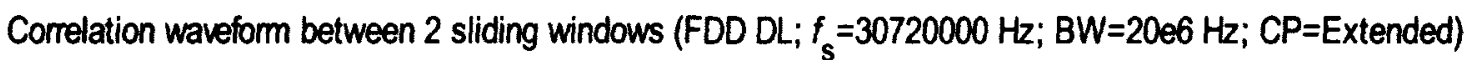

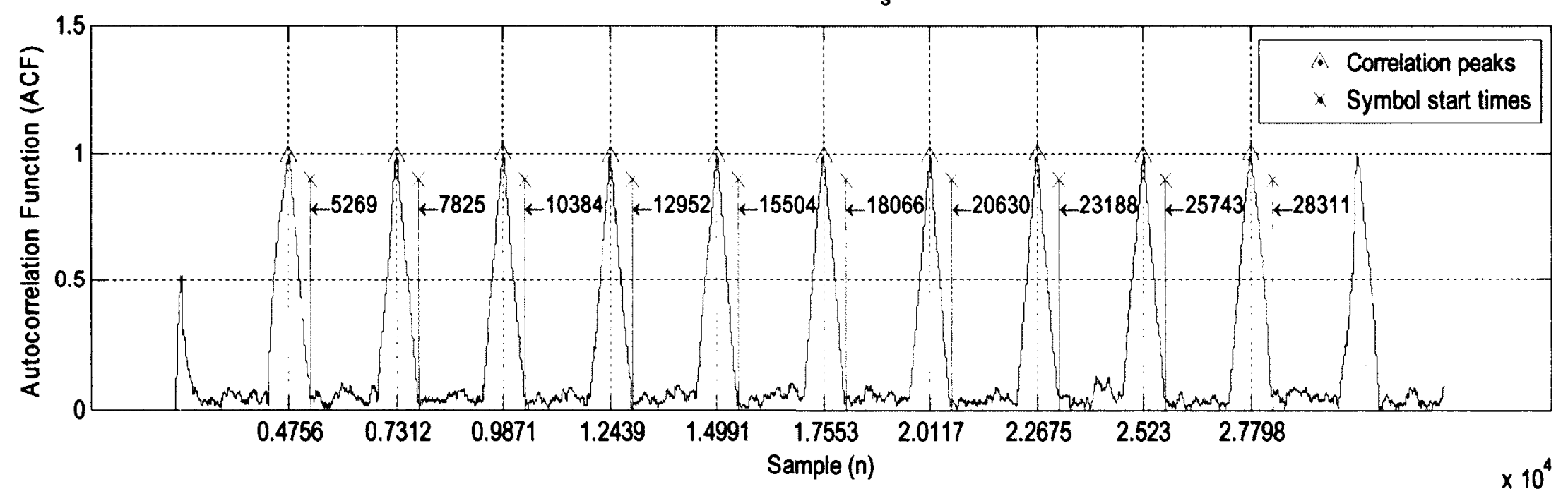

Figure 5.25. Symbol Time Offset (STO) Estimation 20 MHz Downlink CP=Extended 


\subsection{Carrier Frequency Ofiset Estimates Experimental Results}

This section describes the experimental implementation carrier frequency offset estimation algorithm in Matlab based on LTE I/Q waveforms. Frequency synchronization is related to the correction/elimination of the carrier frequency offset (CFO) caused by the mismatch from the RF local oscillator $\left(f_{c}\right)$ and the Doppler shift $\left(f_{\mathrm{D}}\right)$. Carrier frequency offset estimation can be performed in two steps: coarse CFO estimation and fine CFO estimation. Coarse CFO estimation is the process of estimating the subcarrier numbering index $k_{0}$. Fine CFO estimation is the process of estimating the center frequencies of each subcarrier $\varepsilon$. Timing errors of the STO result in intersymbol interference (ISI), whereas imperfect frequency estimations of the CFO lead to intercarrier interference (ICI). The goal in carrier frequency offset (CFO) estimation is to maintain or preserve the orthogonality properties of the sub-carriers, since a frequency error results in inter-carrier interference.

In the Matlab implementation, if we assume that the channel effect is negligible, the phase difference between the cyclic prefix (CP) and the corresponding rear part of an OFDM symbol (spaced $N$ samples away) caused by the CFO $\varepsilon$ is $2 \pi N \varepsilon / \mathrm{N}=2 \pi \varepsilon$. Then the CFO can be found from the phase angle of the product of the cyclic prefix (CP) and the corresponding rear part of an OFDM symbol. In Matlab, the $\mathrm{CFO}$ estimates can be expressed as CFO_CP_est = angle(sliding_w(1,:)*sliding_w $\left.(2,:)^{\prime}\right) /\left(2{ }^{\star} p i\right)$ for the cyclic prefix-based method, CFO_Pream_est $=\operatorname{angle}\left(Y(2,:)^{\star} Y(1,:)^{\prime}\right) /\left(2^{\star} p i\right)$ for the preamble-based method, and CFO_Pilot_est $=\operatorname{angle}\left(Y p(2, k k) \cdot{ }^{\star X p}{ }^{\star}\left(Y p(1, k k) \cdot{ }^{*} X p\right)^{\prime}\right) /\left(2{ }^{*} p i\right)$ for the pilot CAZAC-based method.

Appendix $C$ shows the experimental carrier frequency offset (CFO) estimates, based on LTE I/Q waveforms using $1.4 \mathrm{MHz}, 10 \mathrm{MHz}$, and $20 \mathrm{MHz}$ bandwidths for both normal and extended cyclic prefix. An example of the experimental carrier frequency offset (CFO) estimation output is shown below. 


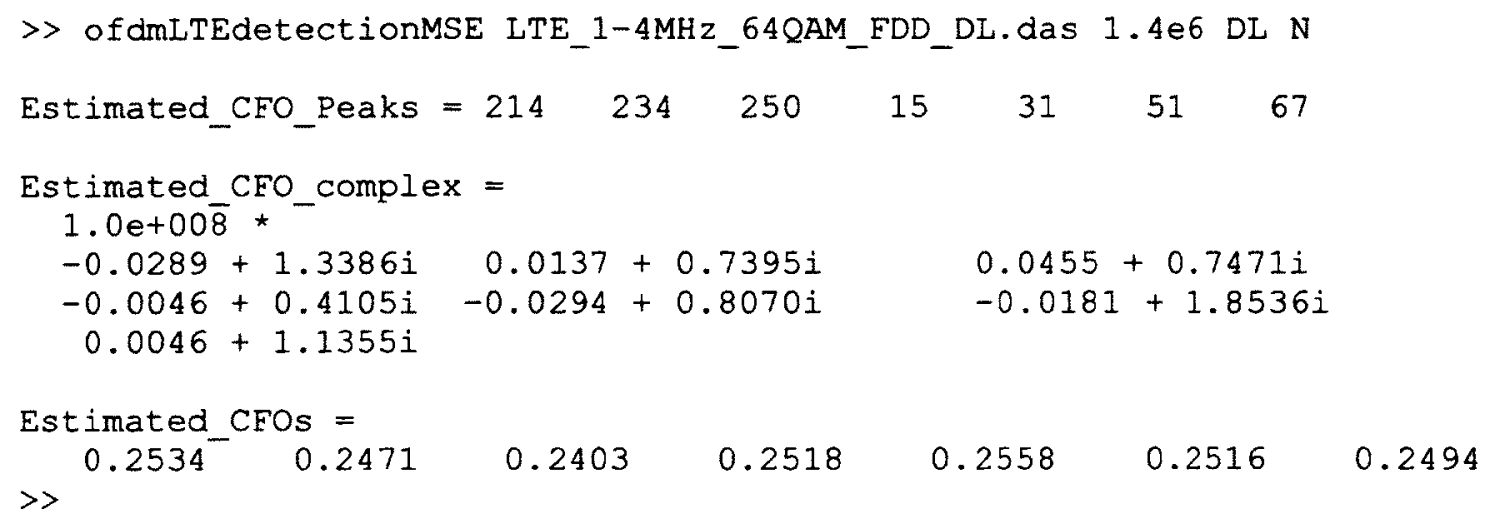




\subsection{Algorithms and MSE vs. SNR Performance Results}

This section discusses the performance efficiency and describes the experimental testing of the proposed pilot-based Zadoff-Chu CAZAC LTE-OFDM synchronization algorithm as compared to other synchronization algorithms, namely the cyclic prefix-based method and the preamble-based method using the Mean Square Error (MSE) vs. SNR estimator.

Figures 5.26 to 5.31 and Figures 5.32 to 5.37 show the MSE (Mean Square Error) performance versus SNR comparison for the three synchronization techniques: the cyclic prefix-based method, the preamblebased method, and the proposed pilot CAZAC-based methods without CFO $(\varepsilon=0)$ and with CFO $(\varepsilon=0.25)$.

For a $1.4 \mathrm{MHz}$ downlink using the normal cyclic prefix case, when a carrier frequency offset is set to $\mathrm{CFO}=0.25$, compared to the carrier frequency offset case of $\mathrm{CFO}=0$, we can observed that the MSE performance degrades from $2.5 \times 10^{-2}$ to $5 \times 10^{-2}$ at a $\mathrm{SNR}=0 \mathrm{~dB}$ using the proposed method. In comparison, the cyclic prefix-based method MSE has degraded from $6 \times 10^{-2}$ to $1.6 \times 10^{-1}$ at a $\mathrm{SNR}=0 \mathrm{~dB}$, whereas the preamble-based method MSE has degraded from $7 \times 10^{-2}$ to $1.3 \times 10^{-1}$ at a SNR=0dB.

For a $20 \mathrm{MHz}$ downlink using normal cyclic prefix case, when the induced a carrier frequency offset is set to $\mathrm{CFO}=0.25$, compared to the carrier frequency offset case of $\mathrm{CFO}=0$, we can observed that the MSE performance degrades from $4.3 \times 10^{-3}$ to $3.8 \times 10^{-3}$ at a $\mathrm{SNR}=15 \mathrm{~dB}$ using the proposed method. In comparison, the cyclic prefix-based method MSE has degraded from $4.2 \times 10^{-2}$ to $6.6 \times 10^{-2}$ at a $\mathrm{SNR}=15 \mathrm{~dB}$, whereas the preamble-based method MSE has stayed the same from $1.75 \times 10^{-2}$ to $1.75 \times 10^{-5}$ at a $\mathrm{SNR}=15 \mathrm{~dB}$.

As expected, as the CFO increases the mean square error degrades; and as the CFO decreases MSE improves proportionally. Overall we can observed from these figures that the proposed CAZAC-based method has achieved the best MSE error performance compared to the cyclic prefix-based and the preamble-based methods. 


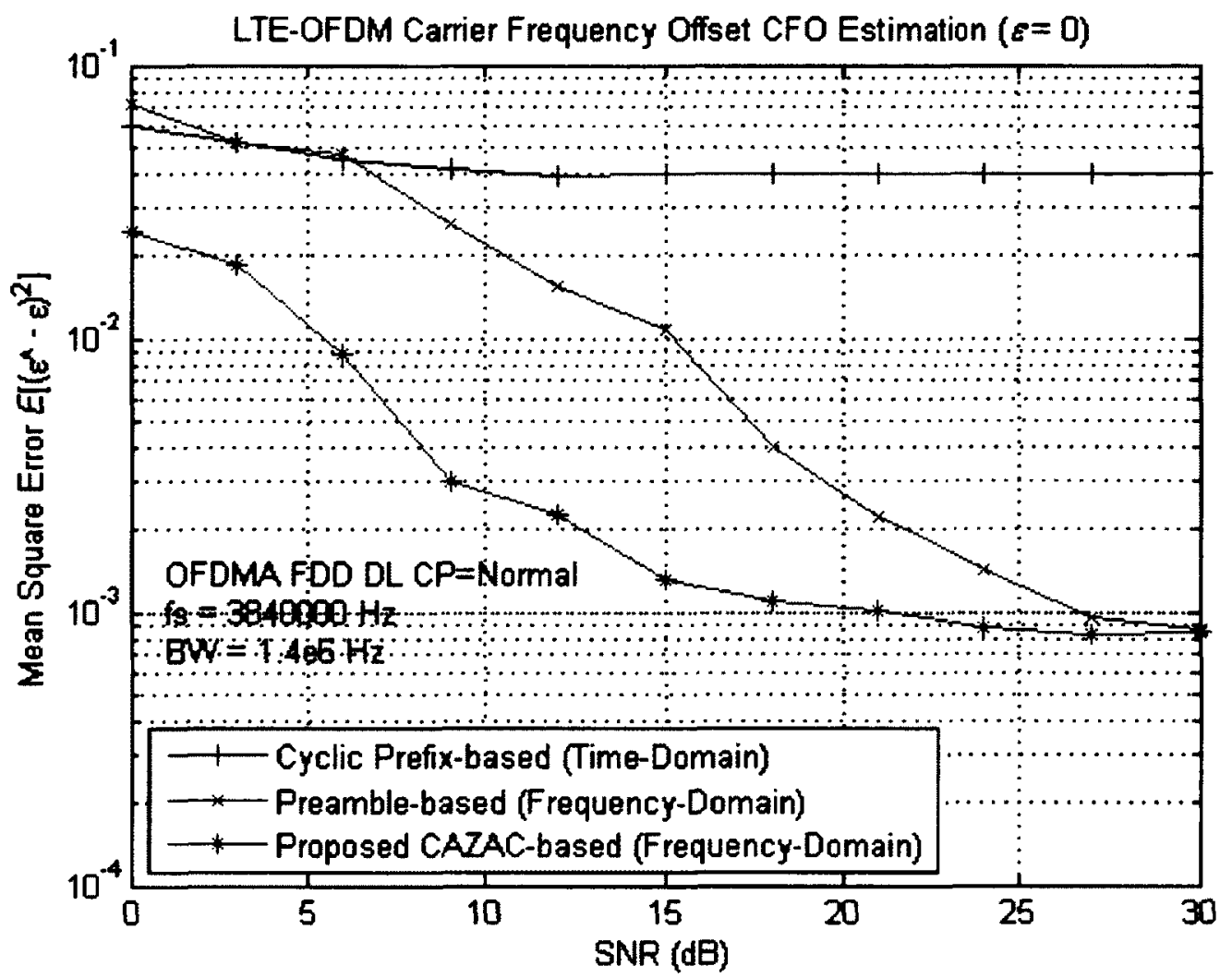

Figure 5.26. Experimental MSE vs. SNR 1.4MHz QPSK CFO $=0$

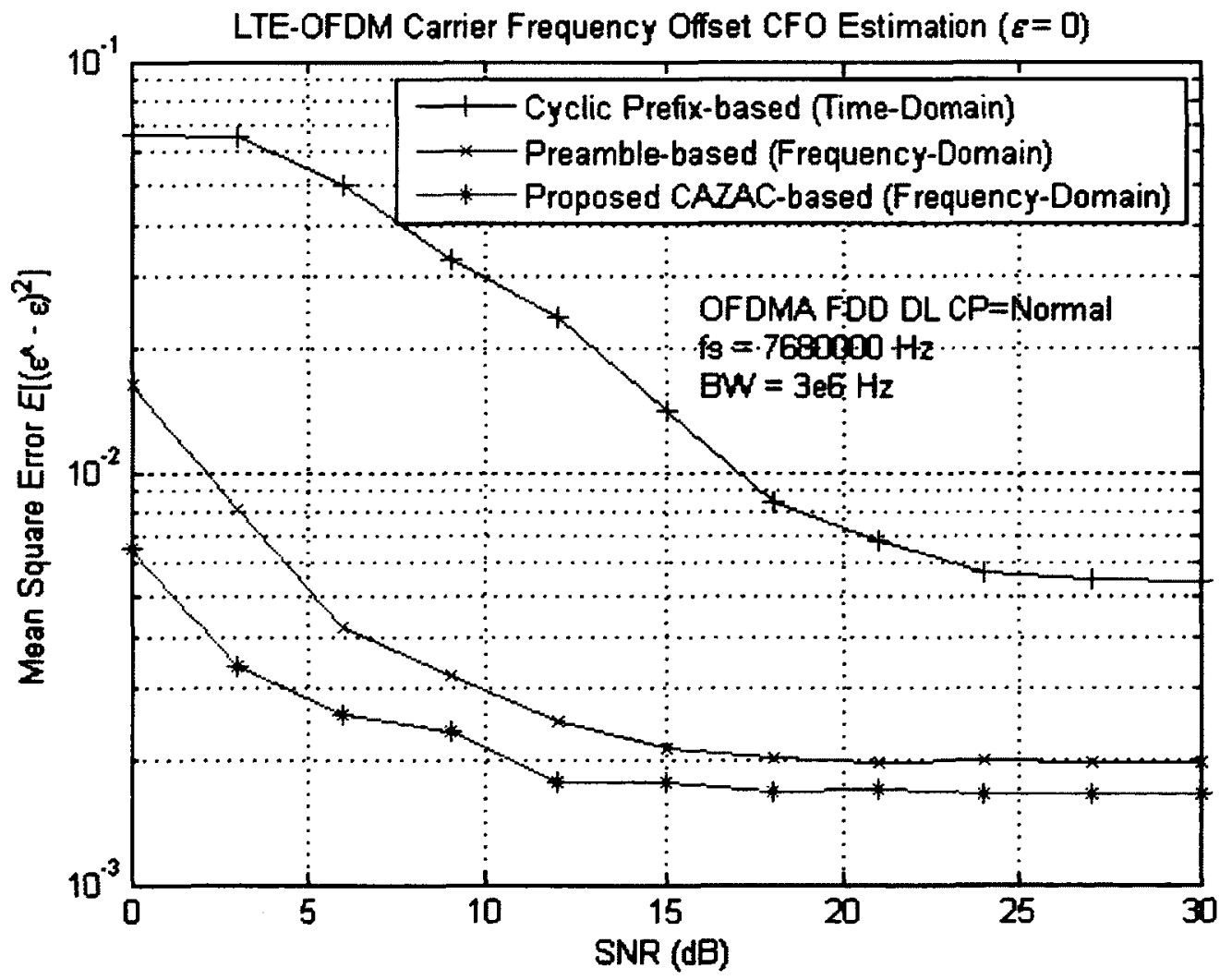

Figure 5.27. Experimental MSE vs. SNR 3MHz 64-QAM CFO =0 


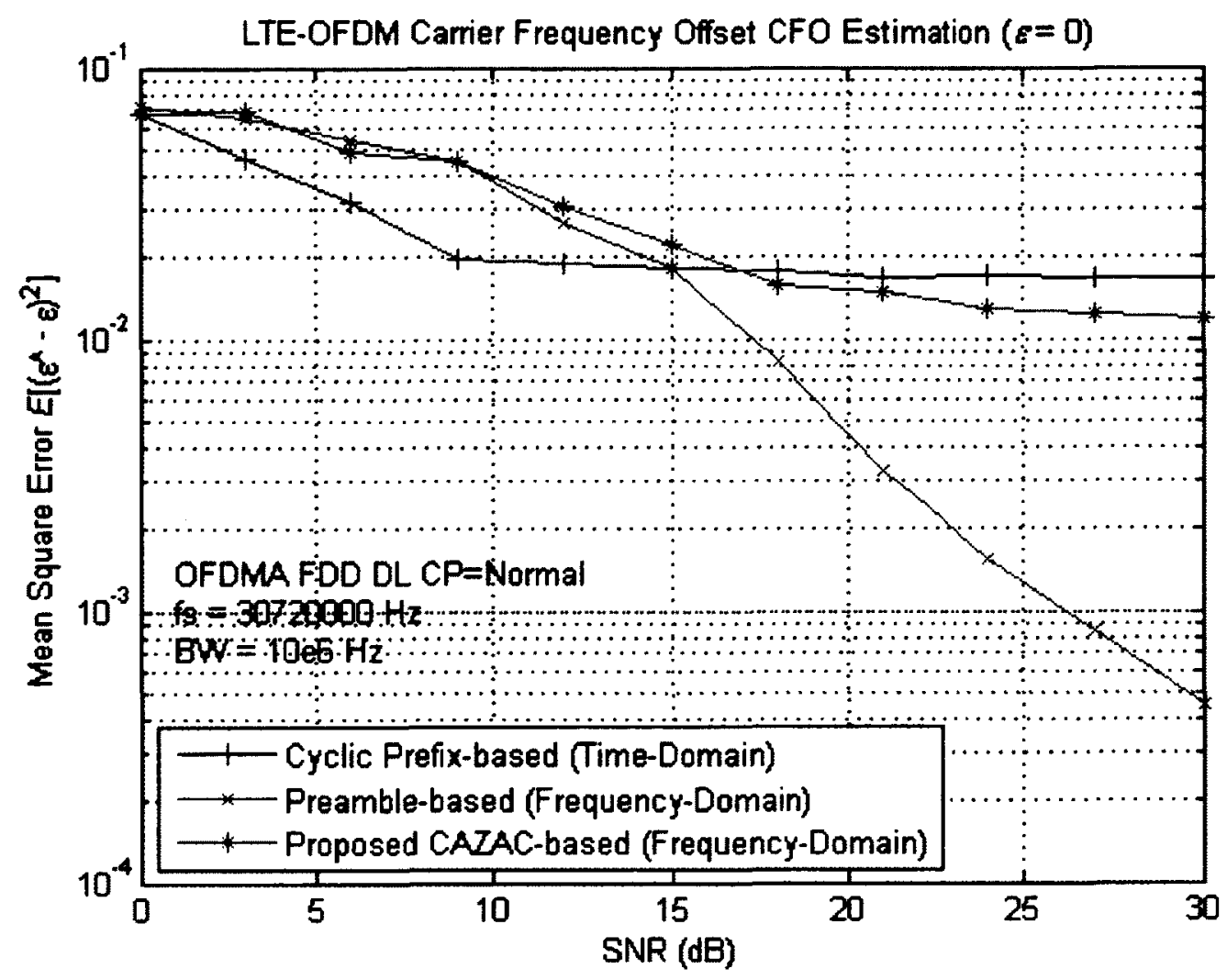

Figure 5.28. Experimental MSE vs. SNR 10MHz 64-QAM CFO $=0$

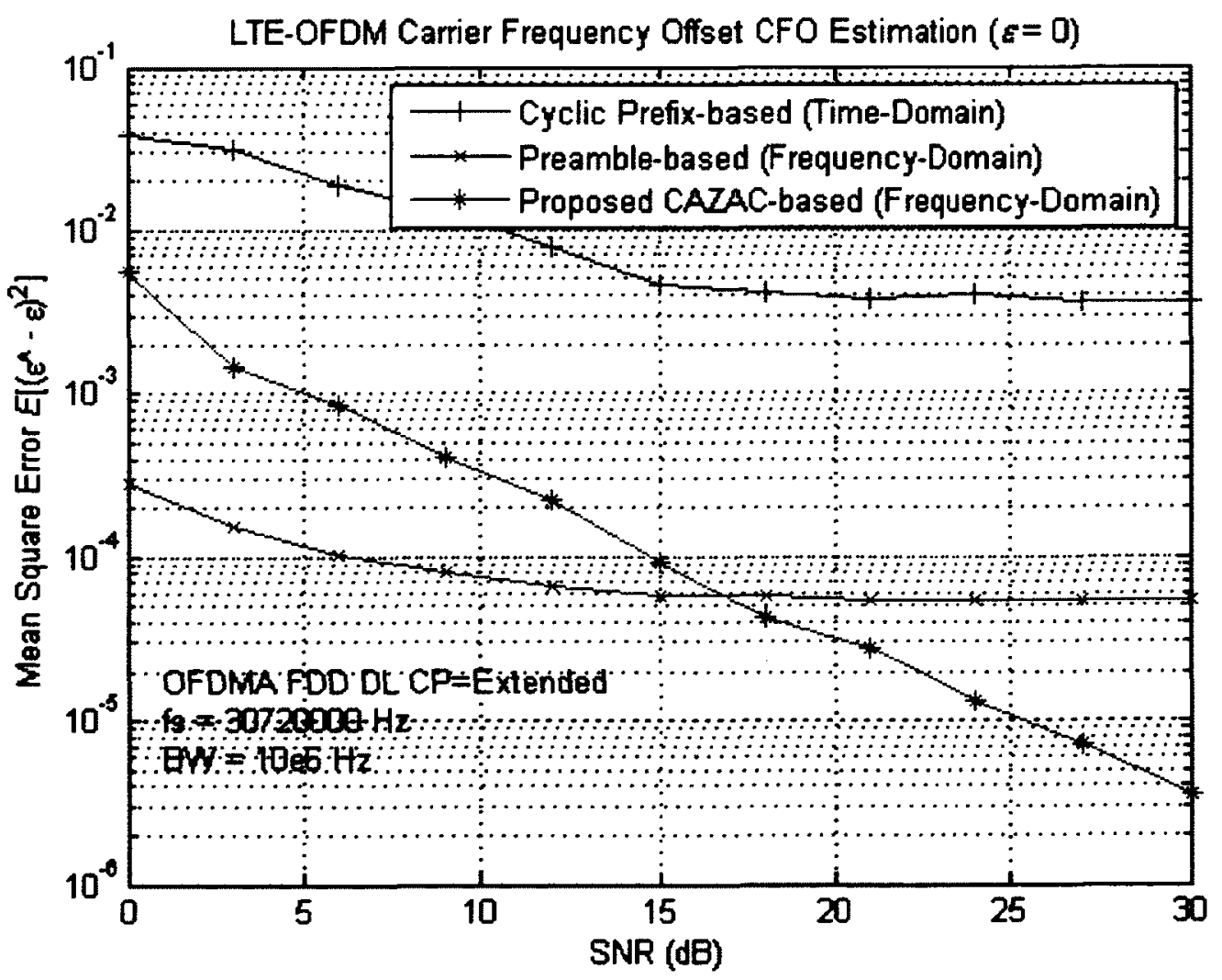

Figure 5.29. Experimental MSE vs. SNR 10MHz 64-QAM CFO = 0 


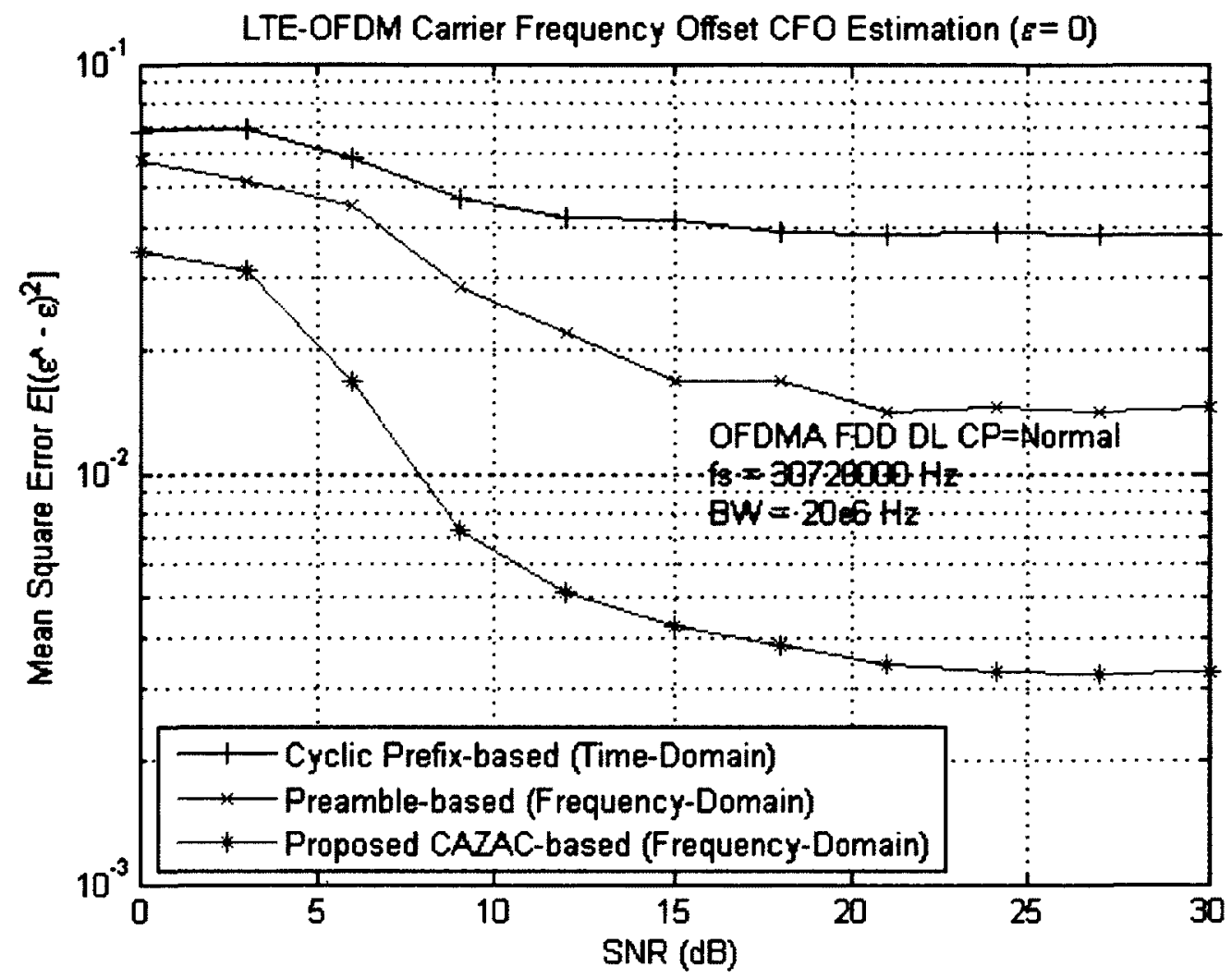

Figure 5.30. Experimental MSE vs. SNR 20MHz 64-QAM CFO $=0$

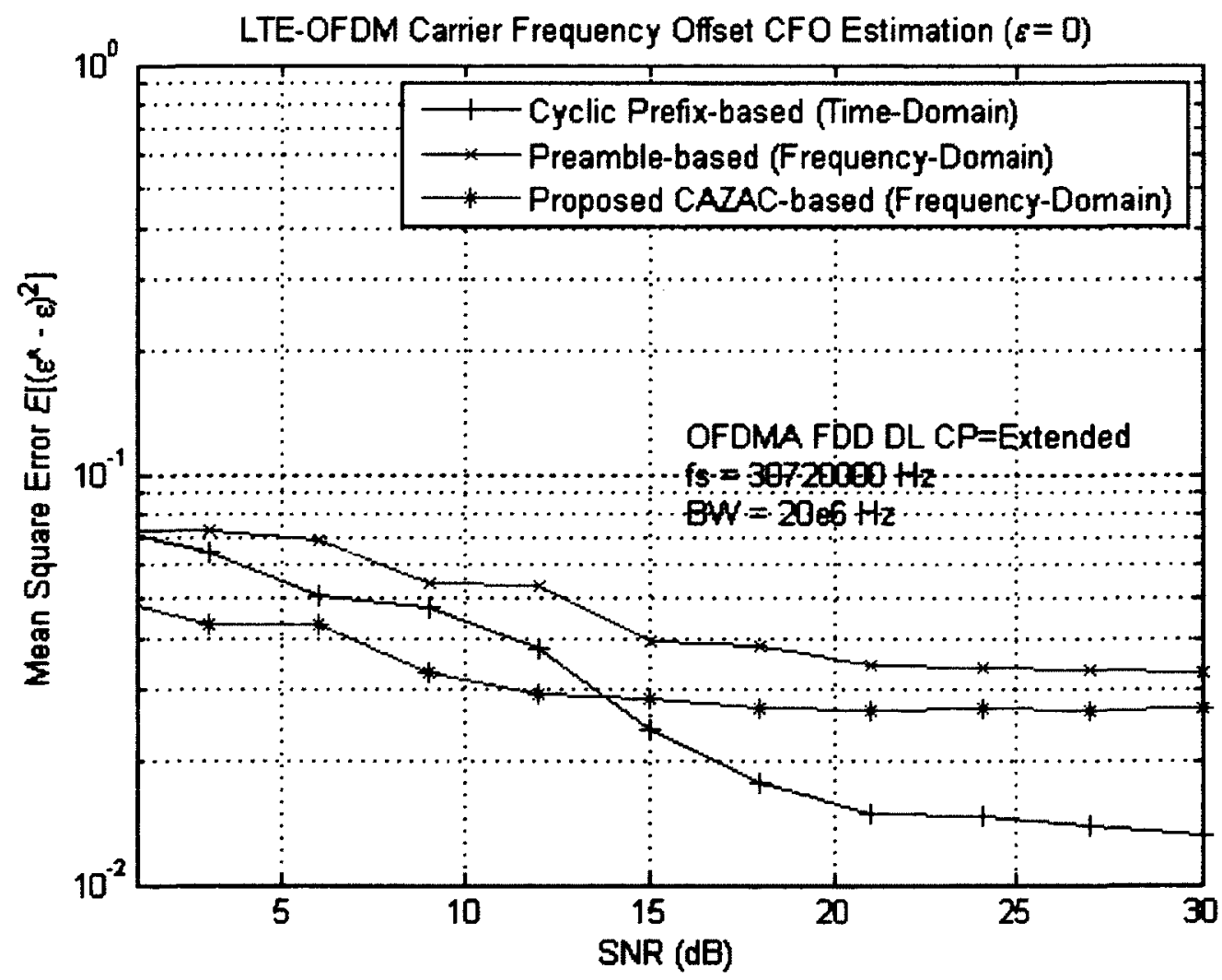

Figure 5.31. Experimental MSE vs. SNR 20MHz 64-QAM CFO = 0 


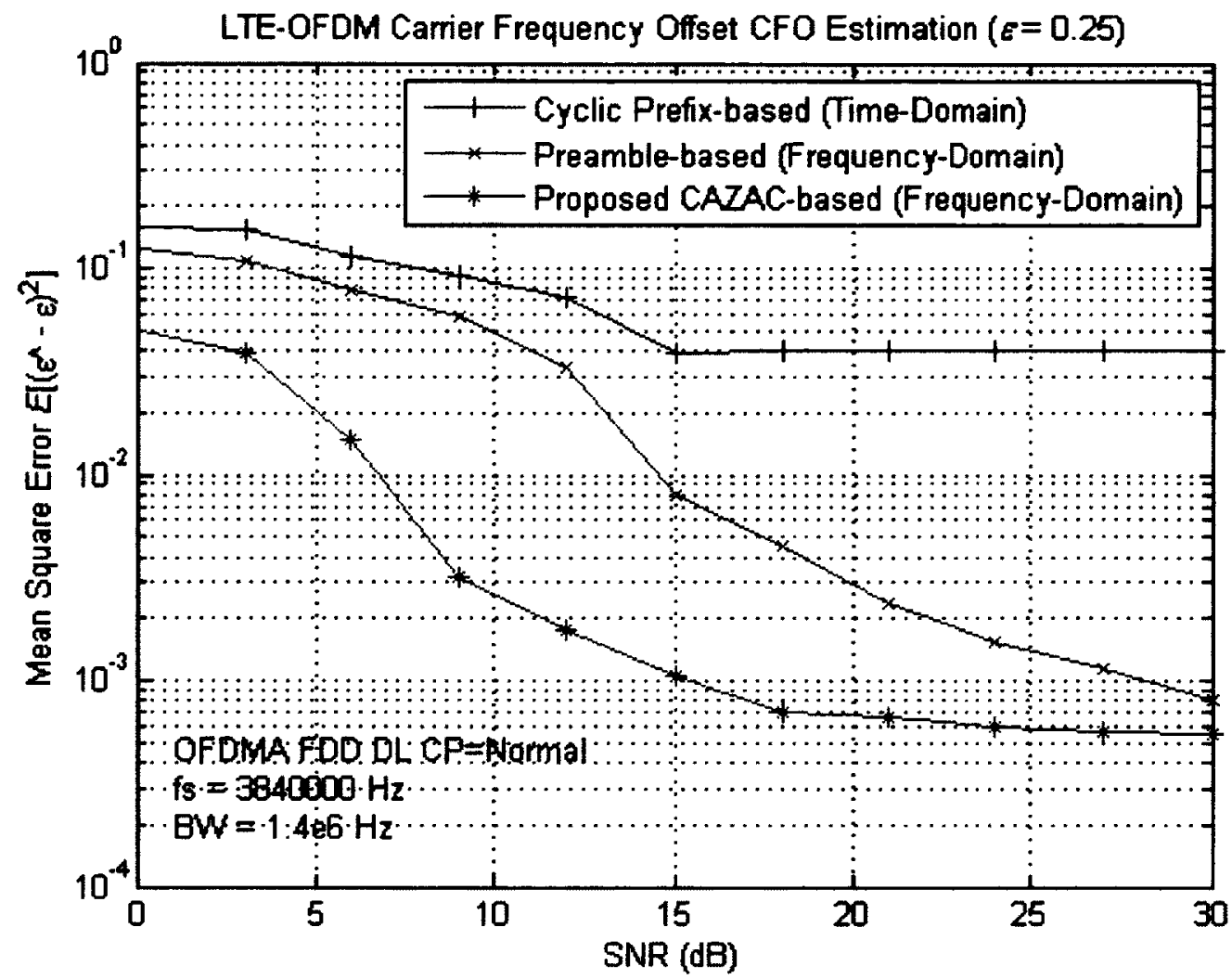

Figure 5.32. Experimental MSE vs. SNR 1.4MHz QPSK CFO $=0.25$

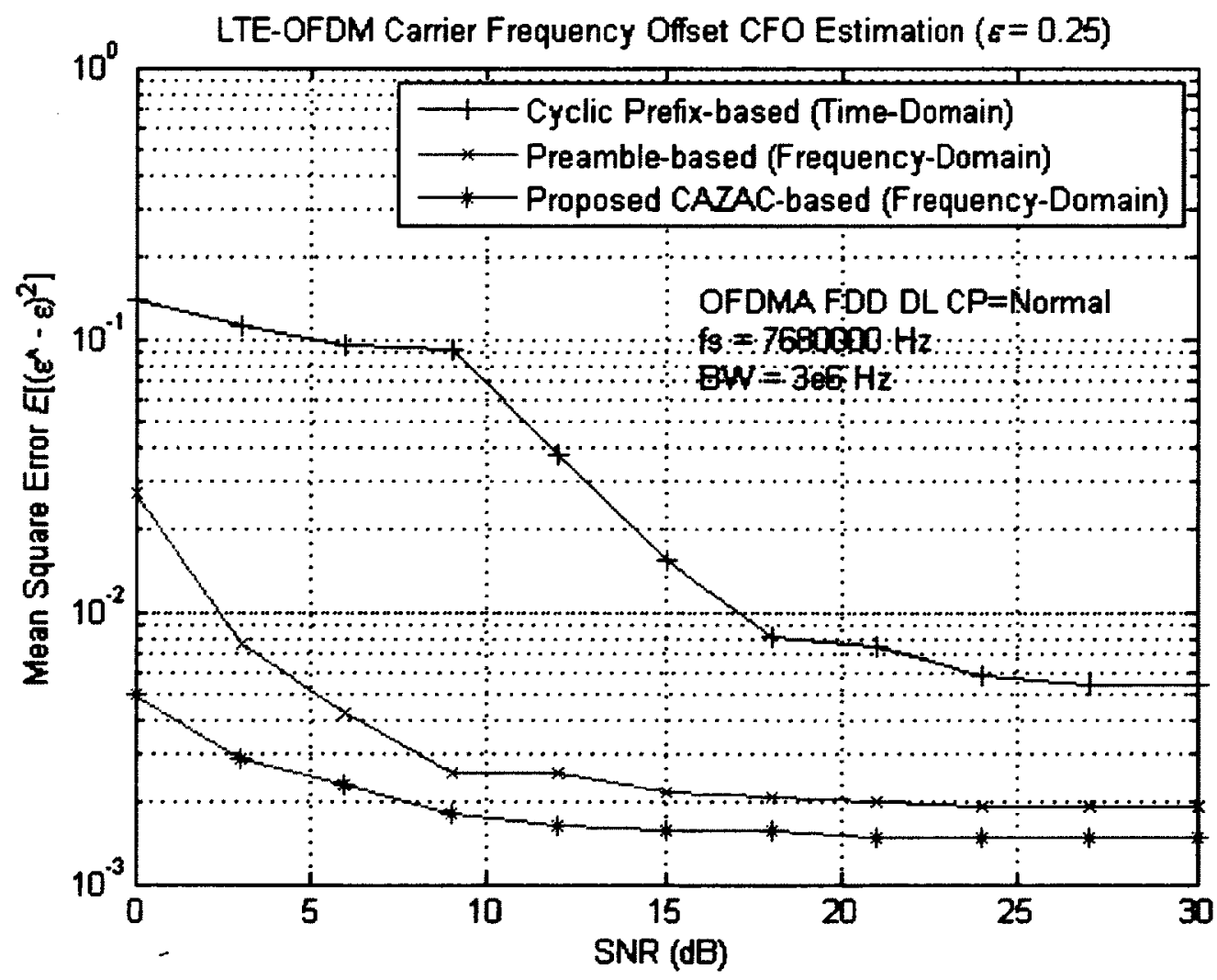

Figure 5.33. Experimental MSE vs. SNR 3MHz 64-QAM CFO $=0.25$ 


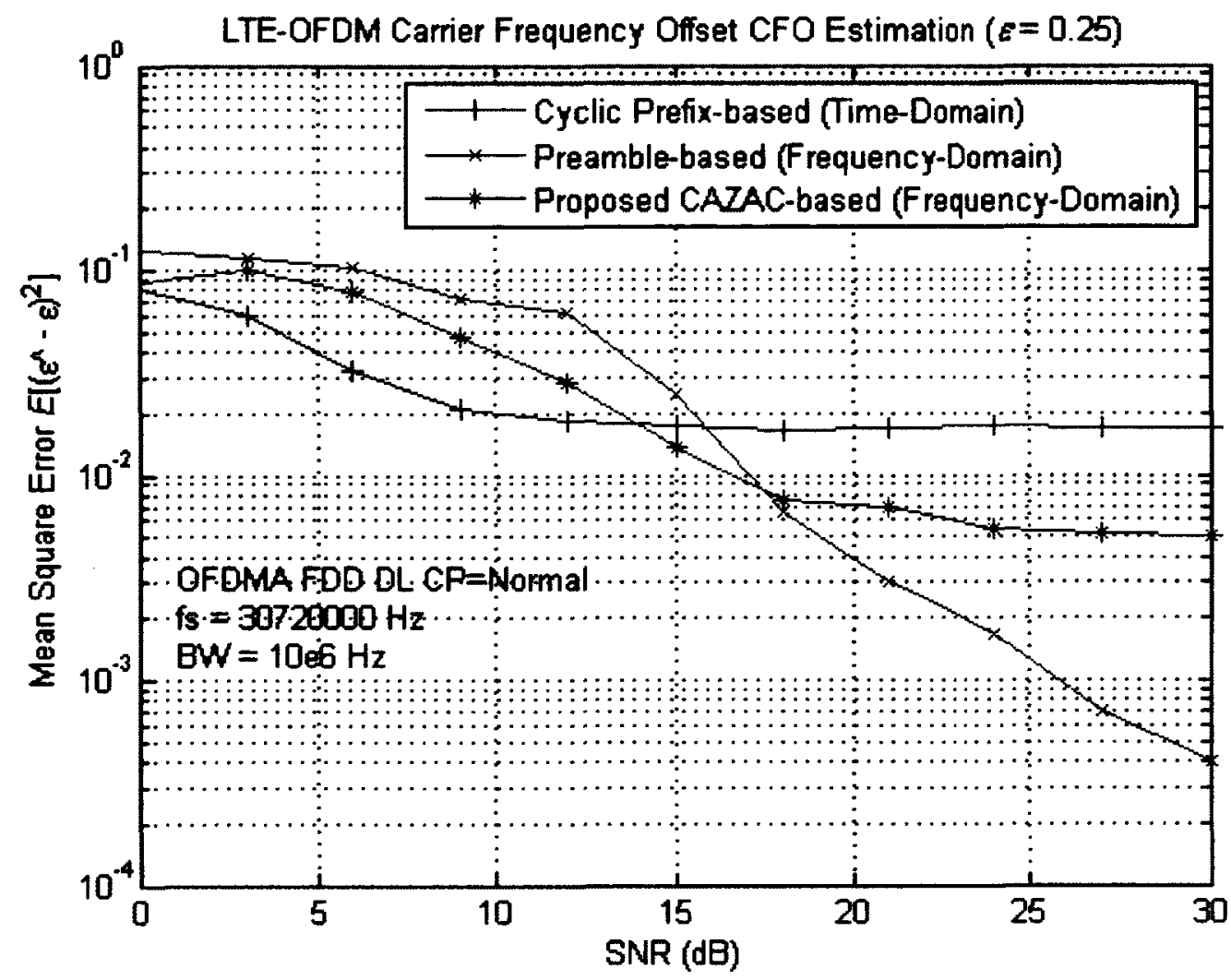

Figure 5.34. Experimental MSE vs. SNR 10MHz 64-QAM CFO $=0.25$

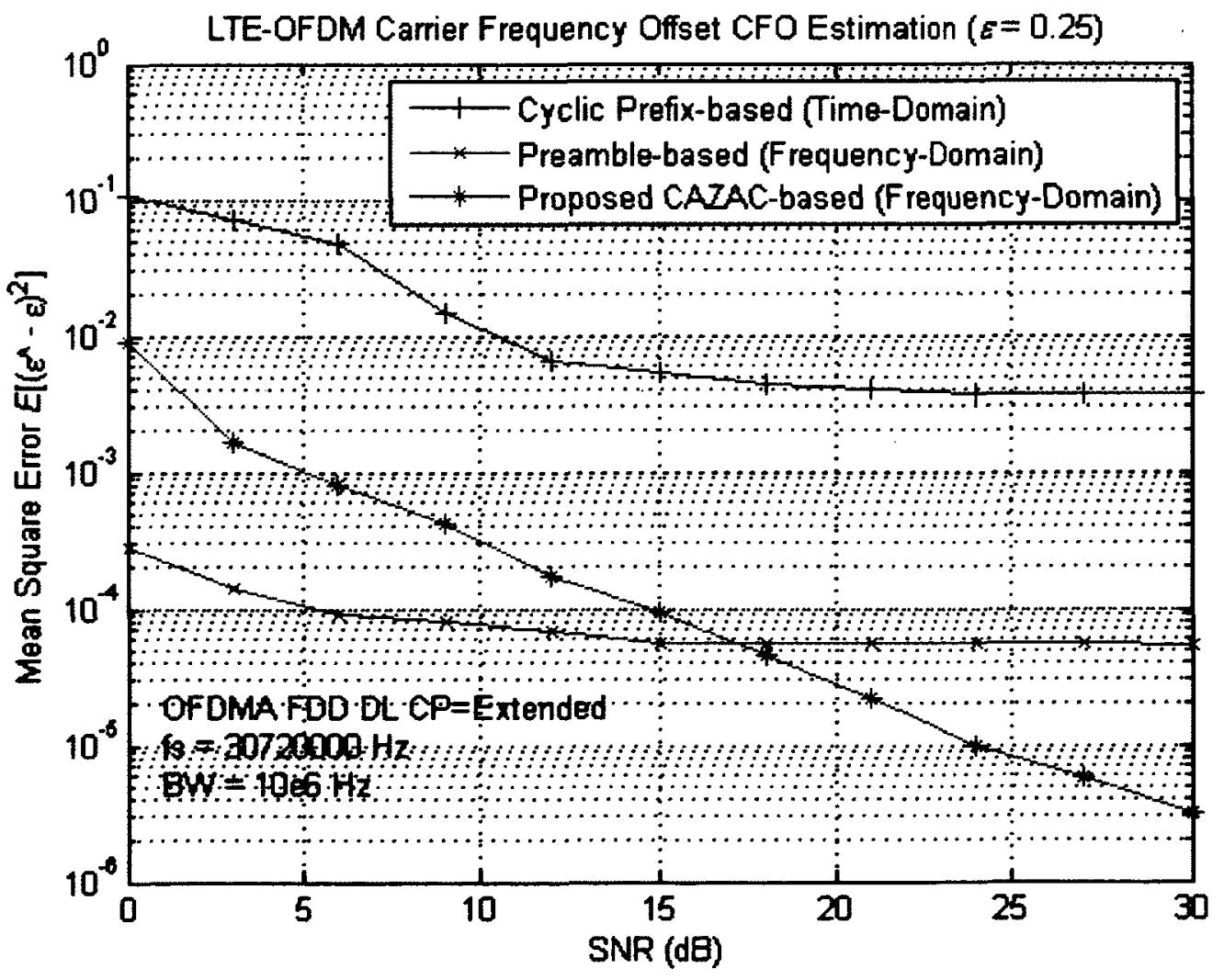

Figure 5.35. Experimental MSE vs. SNR 10MHz 64-QAM CFO $=0.25$ 


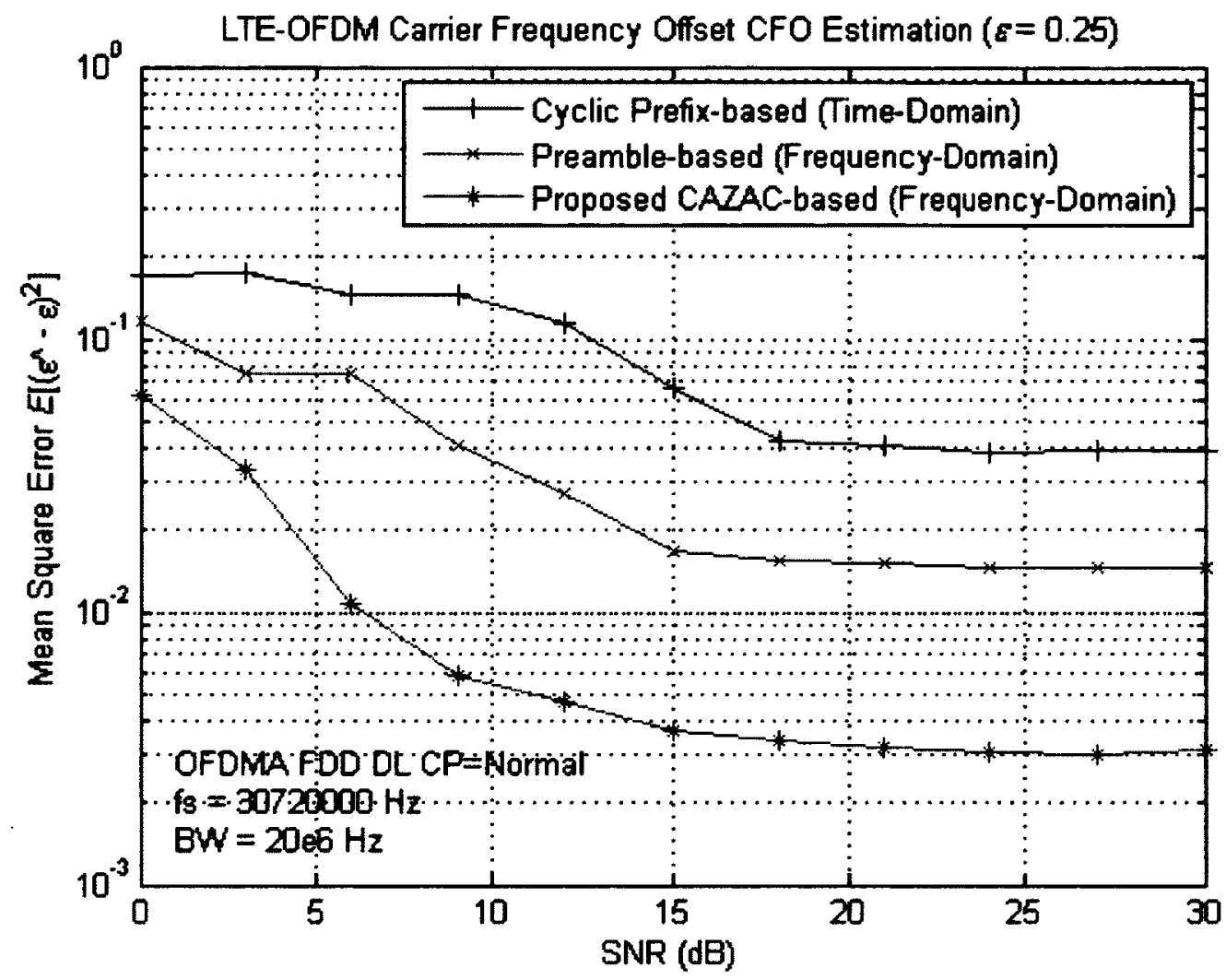

Figure 5.36. Experimental MSE vs. SNR 20MHz 64-QAM CFO $=0.25$

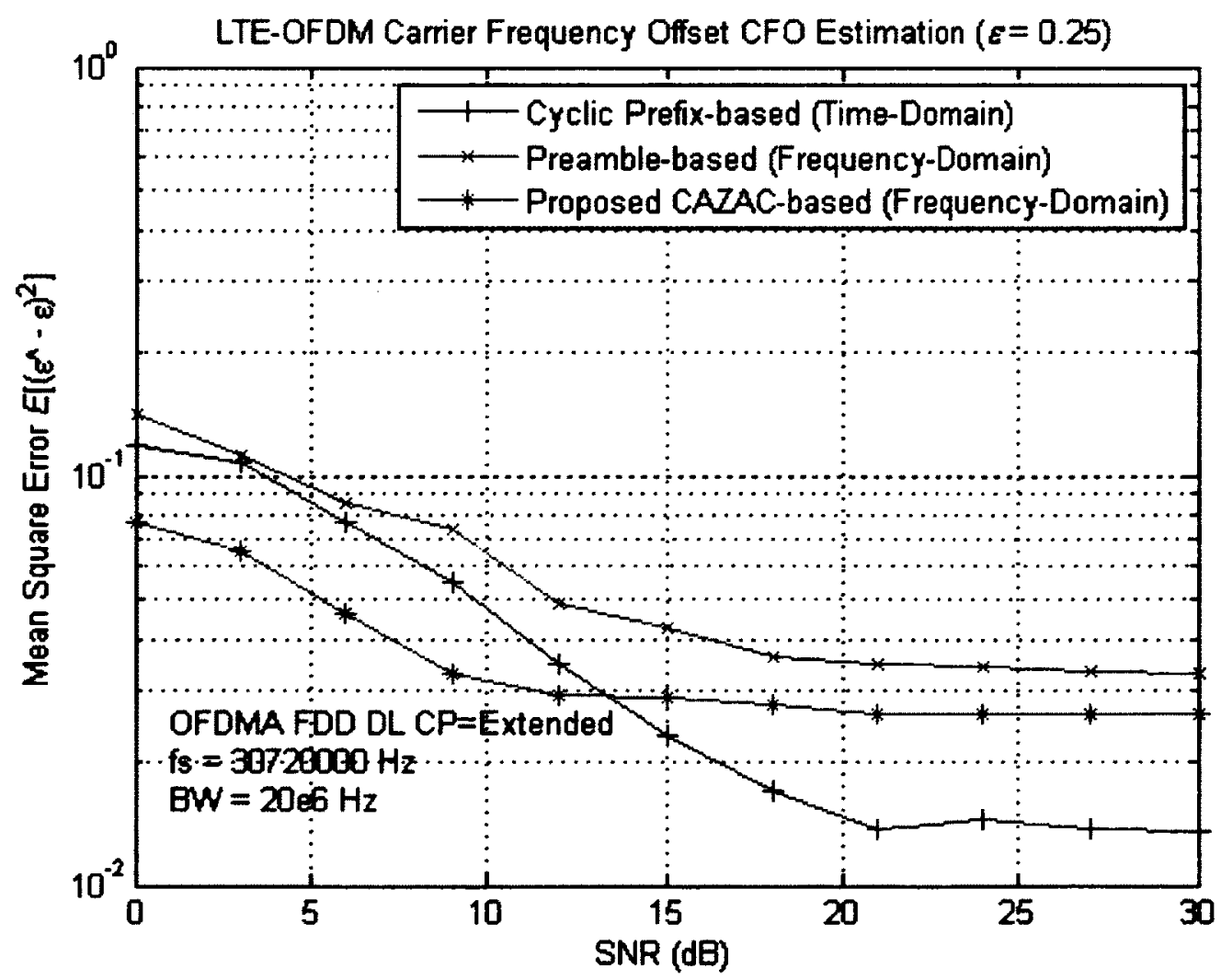

Figure 5.37. Experimental MSE vs. SNR 20MHz 64-QAM CFO $=0.25$ 
Figures 5.38 to 5.43 show the Zadoff-Chu CAZAC sequences for $u=25, u=29$, and $u=34$ which are used as pilot signals in the proposed LTE-OFDM synchronization method in this thesis. The P-SCH signal is transmitted twice in each LTE-OFDM radio frame of $10 \mathrm{~ms}$ duration (i.e., every $5 \mathrm{~ms}$ ) and can provide frame timing synchronization and coarse CFO detection.

As discussed earlier, The P-SCH is composed of a 62-length Zadoff-Chu sequence, $d_{\mathrm{v}}(\mathrm{n})$, generated in the frequency-domain. Zadoff-Chu sequences are specially generated pseudorandom polyphase sequences with perfect correlation properties. These properties make $\mathrm{P}-\mathrm{SCH}$ particularly interesting for obtaining accurate synchronization metrics.

Figures 5.38, 5.40, and 5.42 represents the CAZAC real part and Figures 5.39, 5.41, and 5.43 represents the CAZAC imaginary part of the primary synchronization signal (P-SCH) in the frequency-domain. In LTE frequency division multiplexing (FDD) mode, these signals are located on the 62 subcarriers symmetrically arranged around the DC-carrier.

As we can observed from Figures 5.38 to 5.43 , one characteristic of the CAZAC code is that it has a zero auto-correlation, which means that a CAZAC code is always orthogonal with its cyclic shifted versions and has a constant amplitude.

The major advantages of the CAZAC code include a reduced inter-symbol interference (ISI), minimal interferences between multiple antennas, and a lower PAPR. CAZAC codes are able to achieve better performance with less training symbols, and thus can be used to enhance the channel estimation accuracy, and/or to improve the spectral efficiency, especially when the data field is short.

As a result, CAZAC codes are regarded as optimum training sequences for channel estimation in MIMOOFDM as well as 4G/LTE systems. 


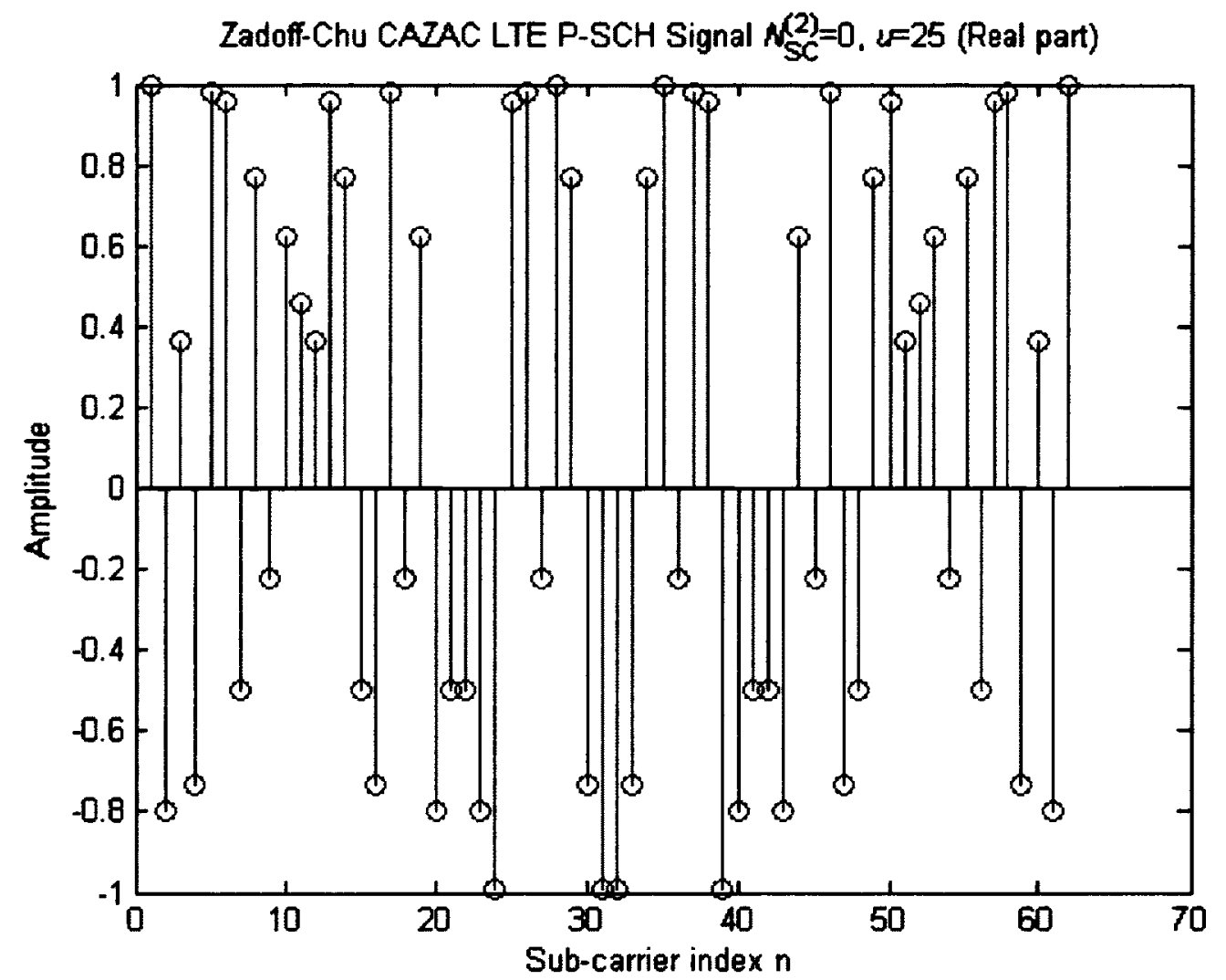

Figure 5.38. LTE P-SCH Zadoff-Chu CAZAC Signal Real Part $(u=25)$

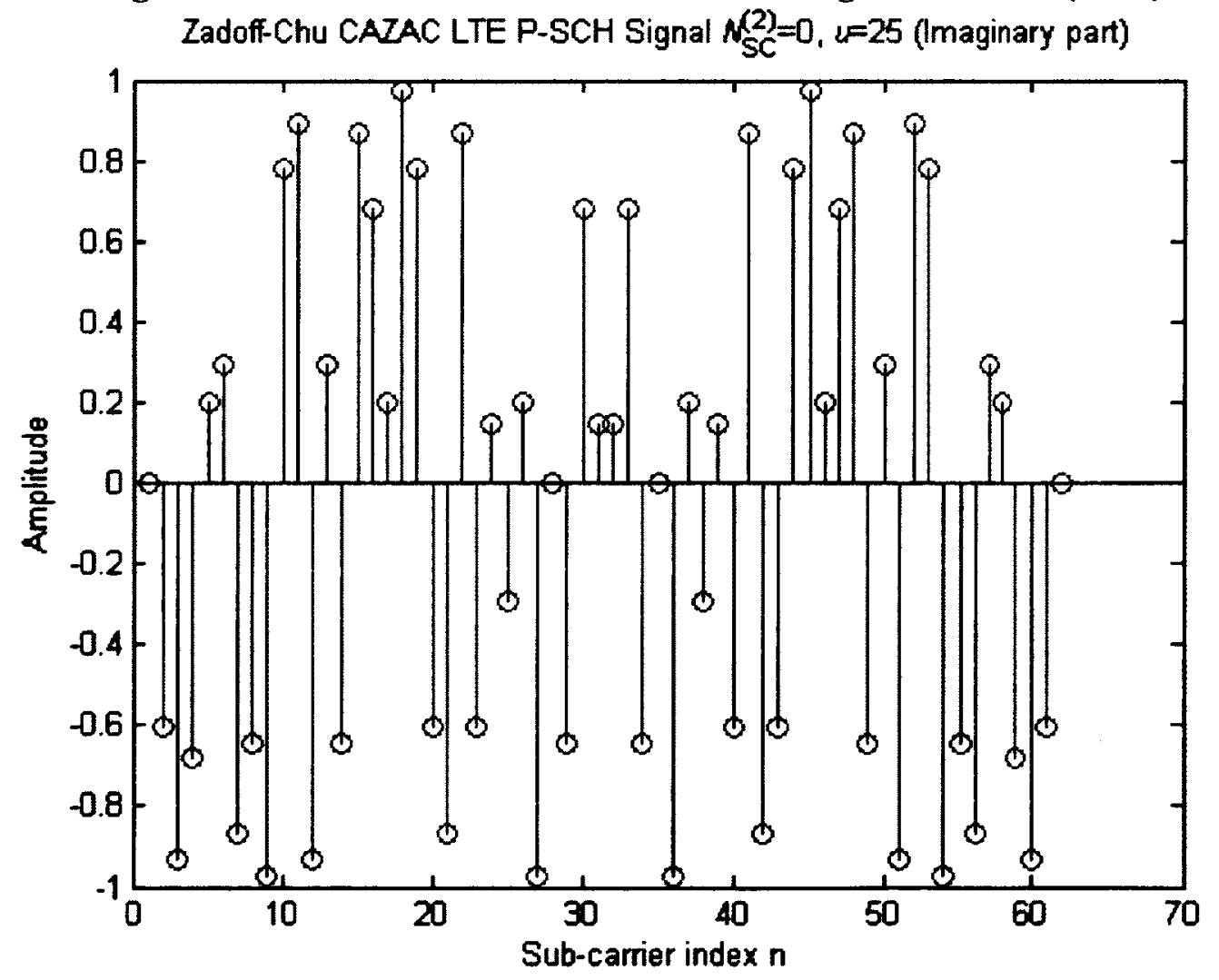

Figure 5.39. LTE P-SCH Zadoff-Chu CAZAC Signal Imaginary Part $(u=25)$ 


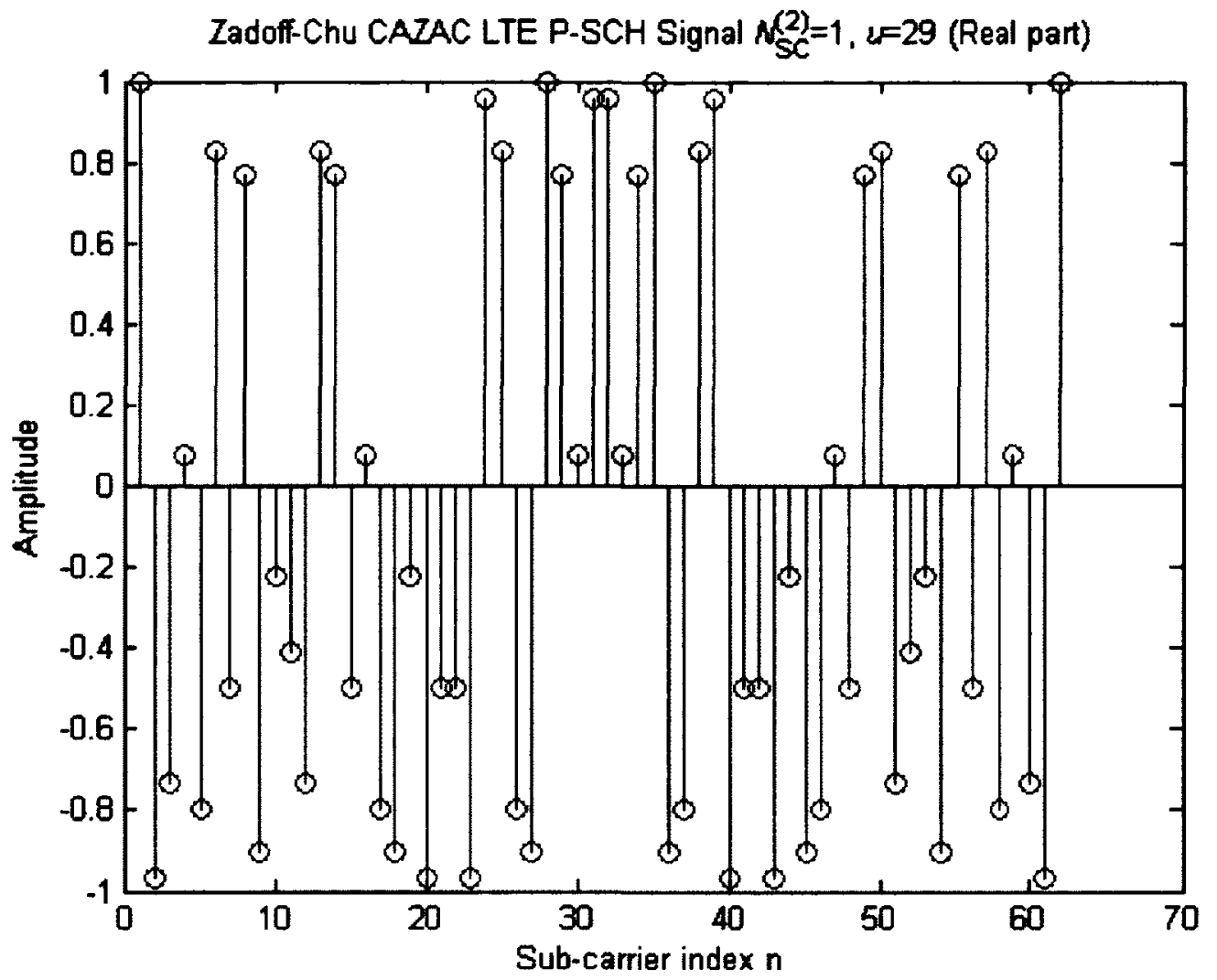

Figure 5.40. LTE P-SCH Zadoff-Chu CAZAC Signal Real Part $(u=29)$

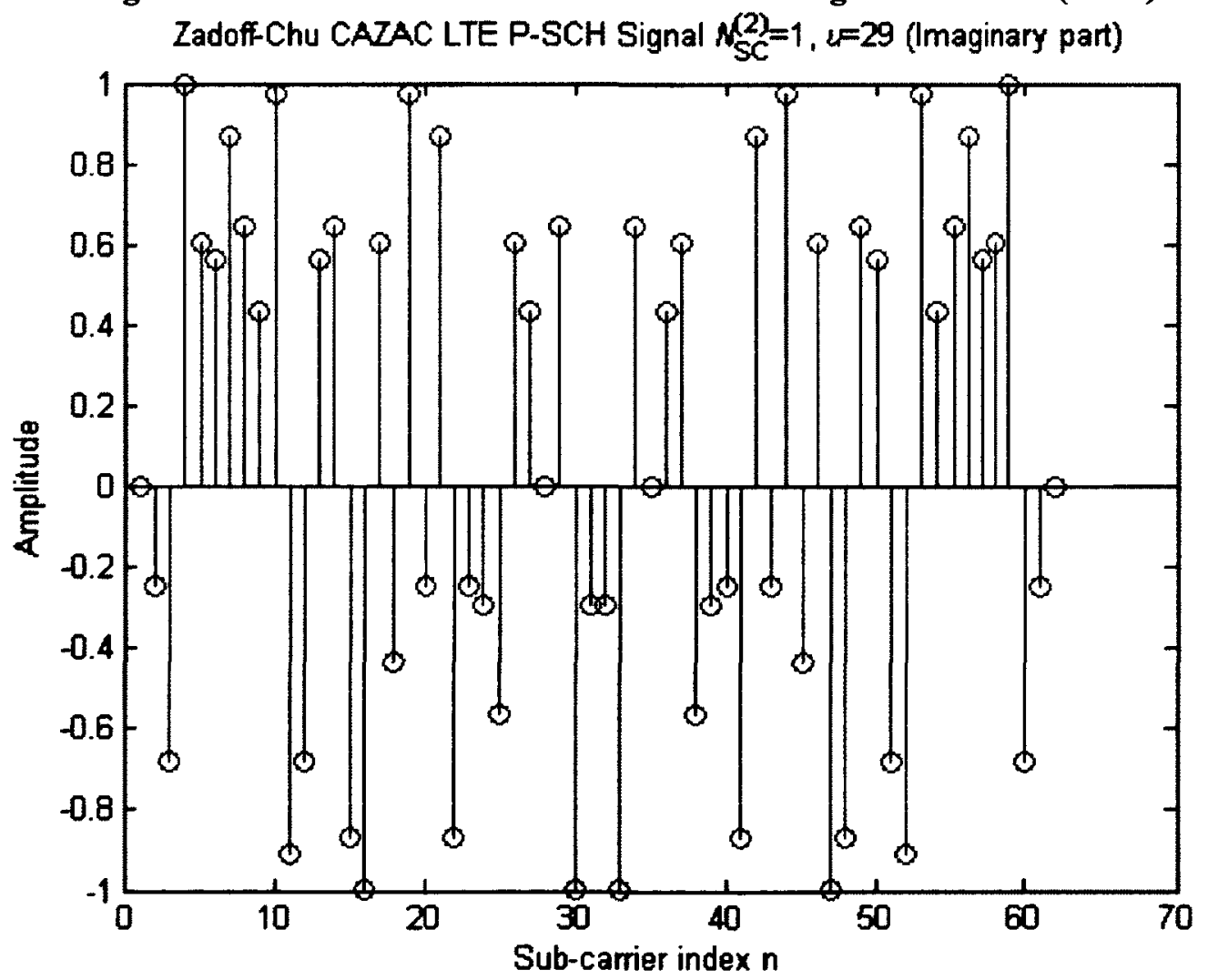

Figure 5.41. LTE P-SCH Zadoff-Chu CAZAC Signal Imaginary Part $(u=29)$ 


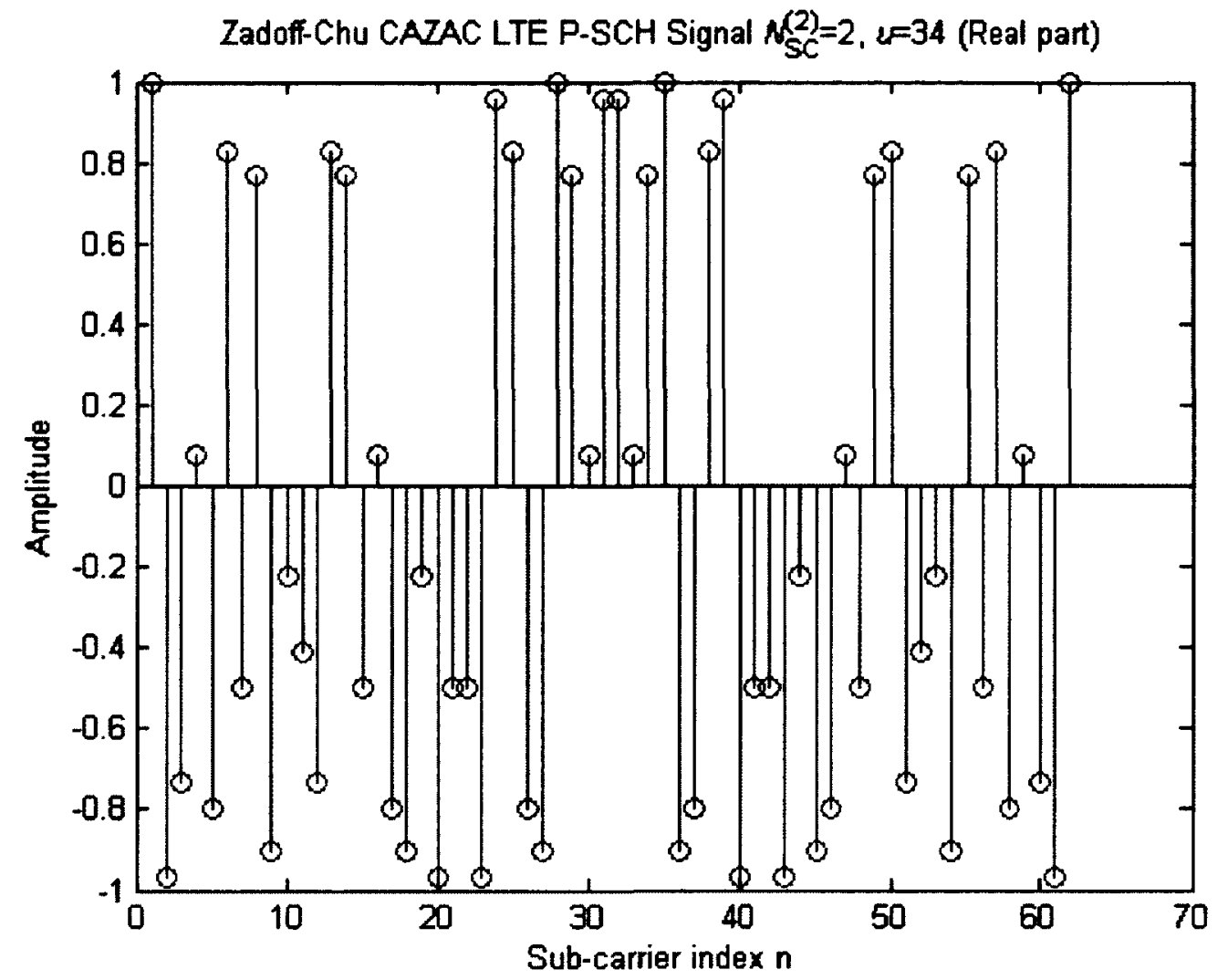

Figure 5.42. LTE P-SCH Zadoff-Chu CAZAC Signal Real Part $(u=34)$ Zadoff-Chu CAZAC LTE P-SCH Signal $M_{S C}^{(2)}=2, v=34$ (Imaginary part)

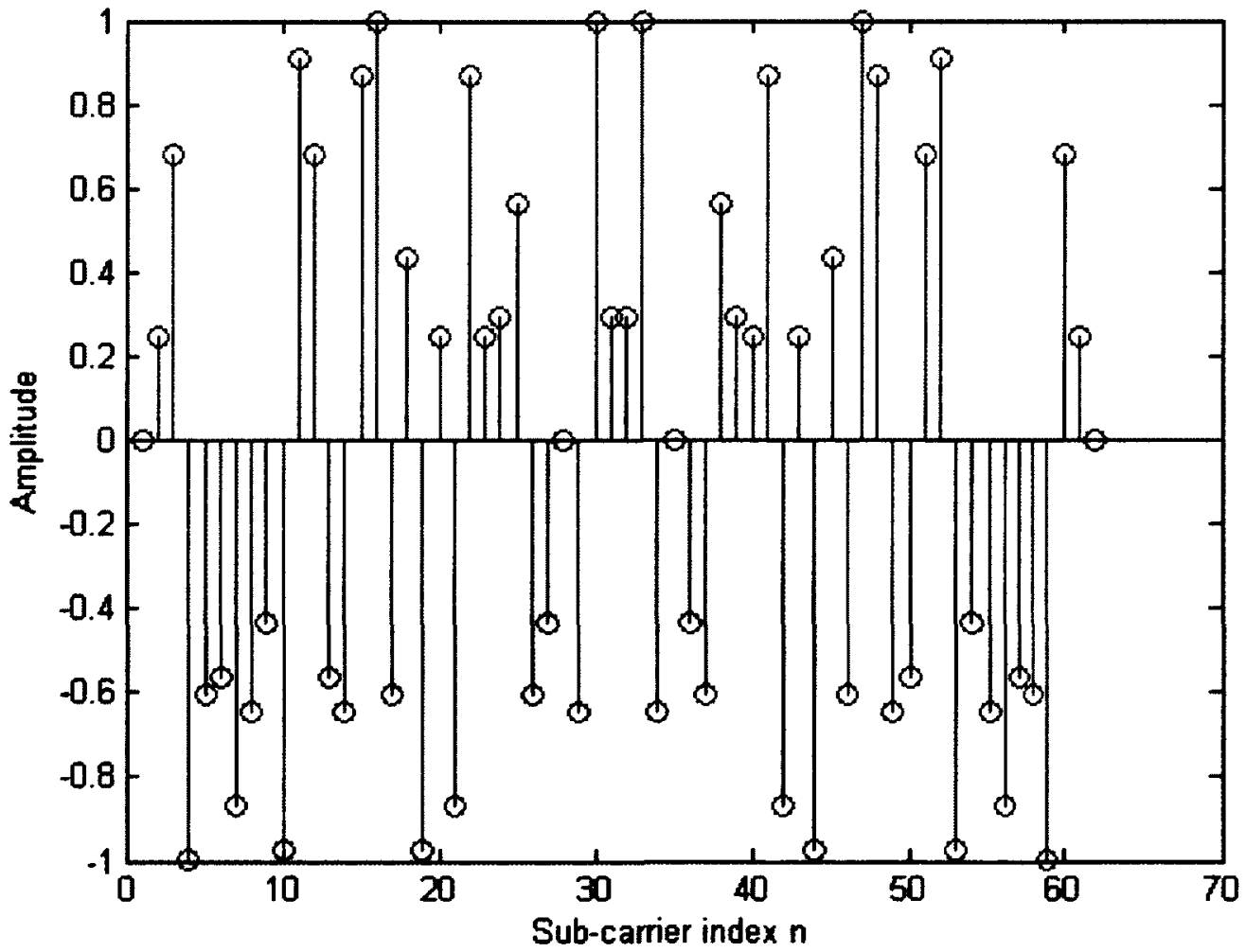

Figure 5.43. LTE P-SCH Zadoff-Chu CAZAC Signal Imaginary Part ( $u=34)$ 


\subsection{Conclusion}

In summary, an experimental LTE synchronization algorithm have been developed and implemented in Matlab where symbol timing offset (STO) and carrier frequency offset (CFO) are estimated using real LTE-OFDM FDD I/Q waveform data.

LTE-OFDM signals have been configured and generated with different scalable bandwidths: $1.4 \mathrm{MHz}$, $3 \mathrm{MHz}, 5 \mathrm{MHz}, 10 \mathrm{MHz}, 15 \mathrm{MHz}$, and $20 \mathrm{MHz}$, in both uplink (OFDMA) and downlink (SC-FDMA) and with Normal as well as Extended cyclic prefixes. The signals have been experimented tested using a block with 262144 points.

In addition, bit-error rate and symbol-error rate (BER vs. $E_{b} / N_{0}$ and SER vs. $E_{b} / N_{0}$ ) error performances have been simulated for LTE modulation schemes (QPSK, 16-QAM, 64-QAM) in both AWGN channel and frequency-selective Rayleigh/Ricean fading channels.

Several synchronization algorithms namely, cyclic-prefix based, preamble-based (two identical OFDM symbols), and CAZAC Zadoff-Chu pilot-based have been analyzed, simulated, experimentally tested, and compared using the mean square error (MSE).

The proposed Zadoff-Chu CAZAC-based method has achieved the best MSE error performance, and outperformed the cyclic prefix-based and preamble-based methods. In the Classen \& Meyr97 method, the size of the cyclic prefix is extended to make the non-ISI portion of the cyclic prefix larger. Pilot symbols are used to mitigate the effects of rapid fading in mobile communications. Pilot-assisted synchronization and diversity reception techniques are used to combat the effects of fading and dispersion in a wireless channel. 


\section{Chapter 6 . Conclusion}

This thesis has investigated several state-of-the-art spectrum sensing methods and the latest research on synchronization techniques for frequency-agile radios from the LTE and OFDM perspective. In particular, two major challenges related to the strict requirements of time and frequency synchronization in multicarrier OFDM, specifically in the context of LTE (3GPP) and LTE-Advanced (4G) were addressed. The first challenge, that of symbol time offset (STO) or time synchronization, consisted of how the receiver is able to determine the exact instant at which the OFDM symbol starts (for the correct positioning of the DFT window). The second challenge, that of frequency synchronization, consisted to eliminating the carrier frequency offset (CFO) caused by the mismatch of the RF local oscillators $\left(f_{C}\right)$ and due to Doppler shift $\left(f_{D}\right)$.

In general, the principle of multi-carrier (MC) orthogonal frequency division multiplex (OFDM) transmission is to convert a serial high-rate data stream to multiple parallel low-rate sub-streams. In OFDM, the cyclic prefix converts linear convolution into circular convolution. Carrier frequency offset (CFO), destroys orthogonality causing inter-carrier interference (ICI) and bit error rate (BER) degradation. In single carrier (SC) transmission BER degradation is dominated by the reduction of the useful signal component, whereas in MC transmission, BER degradation is determined by the ICI.

Chapter 2 presented the theoretical background. OFDM can be seen as either a modulation technique or a multiplexing technique. The requirements for time offset estimate are determined by the difference in length between the cyclic prefix and the channel impulse response. This difference represents the part of the cyclic prefix which is not affected by the previous symbol due to the channel dispersion. As long as a symbol time offset (STO) estimate does not exceed this difference, the orthogonality of the subcarriers is preserved, and a time offset within this interval only results in a phase rotation of the subcarrier 
constellations. Although OFDMA is widely known from several Standards (Wi-Fi, WiMAX, DVB. etc.), SC-FDMA is not yet known from Standards. In an OFDMA downlink, each sub-carrier only carries information related to one specific symbol. In a SC-FDMA uplink, each sub-carrier contains information of all transmitted symbols. The higher the speed of the receiver (mobile station) and the higher the transmission rate, the higher the Doppler shifts will be, and the higher the ICI will be. ICI sets a maximum speed limit at which any given OFDM transmission mode can handle before reception fails.

Chapter 3 presented several spectrum techniques. Spectrum sensing is the first step to implementing a frequency-agile radio system. There are three major categories of spectrum sensing techniques: primary transmitter detection, cooperative detection, and interference-based detection. The cooperative spectrum sensing (CSS) techniques improve reliability and detection capability, mitigate multipath fading and shadowing by spatial diversity, and avoid hidden terminal problem.

Chapter 4 discussed the latest research techniques in OFDM synchronization in the time-domain as well as in the frequency-domain. Most algorithms for symbol or time synchronization are based on the calculation of correlation metrics that exploit some periodicity of the OFDM signal, that is due to the cyclic prefix (Non data-aided) or to a known training sequence or pilot (Data-aided). The advantage of the data-aided methods is a wide frequency offset correction range. The disadvantage of data-aided methods is a loss in data rate throughput since the insertion of pilot symbols usually implies a reduction of the data rate. It should be emphasised that the frequency synchronization at the receiver must be very precise in order to avoid any Inter Carrier Interferences (ICI). Carrier frequency offset (CFO), destroys orthogonality, causes intercarrier interference (ICI), and bit error rate (BER) degradation. In single carrier transmission, BER degradation is dominated by the reduction of the useful signal component, whereas in multicarrier transmission, BER degradation is determined by the ICI. In CFO estimation, there is a tradeoff between acquisition range and resolution. In a single carrier system (SC), a single fade or interferer can cause the entire link to fail, whereas in a multicarrier system (MC), only a small percentage of the 
subcarriers will be affected. Also, the closer the symbol time-offset (STO) estimate is to the true timing offset, the shorter the cyclic prefix needs to be, reducing the overhead in the system. Frequency-domain estimation algorithms often have limitations in their capability and one must ensure that the synchronization errors are within the frequency-domain estimator's limits. Usually, this is achieved through time-domain estimation/compensation of coarse errors. It is noted that short acquisition time leads to lower system complexity and data-aided (DA) approaches, lead to reduced bandwidth efficiency. This is the reason why we have used the CAZAC sequence. Since CAZAC codes are able to achieve better performance with fewer training symbols, they can be used to enhance the channel estimation accuracy, and/or improve the spectral efficiency, especially when the data field is short. As a result, CAZAC codes are regarded as optimum training sequences for channel estimation in MIMO-OFDM systems.

Chapter 5 discussed the experimental/simulation results. High level M-ary schemes (such as 64-QAM) are very bandwidth efficient, but more susceptible to noise and require linear amplification. The combined linear and constant envelope M-ary modulation (QAM) is useful in band-limited channels, it has greater bandwidth efficiency, significantly higher BER, smaller distances in its constellation, and low sensitivity to timing jitter. This is the reason why there are some variations in the MSE vs. SNR performance curves. The proposed pilot-based method or CAZAC-based scheme performs both coarse and fine frequency offset estimation. The CAZAC code has a zero auto-correlation, which means that a CAZAC code is always orthogonal with its cyclic shifted versions and has a constant amplitude. The major advantages are reduced inter-symbol interference (ISI), minimal interferences between multiple antennas, and lower PAPR. CAZAC codes are able to achieve better performance with less training symbols, and thus can be used to enhance the channel estimation accuracy, and/or improve the spectral efficiency, especially when the data field is short. As a result, CAZAC codes are regarded as optimum training sequences for channel estimation for multicarrier MIMO-OFDM systems in frequency-selective fading environment. 


\subsection{Research Contribution}

This research thesis has investigated several state-of-the-art spectrum sensing methods and the latest research on synchronization techniques for frequency-agile radios from the LTE and OFDM perspective. There are four major research contributions made by this thesis. The first contribution is a comprehensive study, investigation, and classification of the latest research on spectrum sensing mechanisms in the spectrum-agile radio context. The second contribution is an experimental study of LTE-OFDM time and frequency synchronization techniques. The third contribution is a proposed OFDM synchronization algorithm based on the Zadoff-Chu CAZAC pilot sequence (i.e. the LTE primary synchronization signal). The proposed CAZAC-based method has achieved the best MSE error performance and has outperformed the cyclic prefix-based and preamble-based methods. The fourth contribution is the development and evaluation of a proof-of-concept demonstrator. In order to replicate the LTE-OFDM realm of waveform creation, transmission, and reception, we have used a combination of four very sophisticated and specialized software packages and hardware instruments: the IQCreator ${ }^{\mathrm{TM}}$ (for LTE-OFDM waveform creation), the Aeroflex IFR 3416 (for RF transmission), the R\&S FSV (for BB reception), and the CRC Spectrum Explorer ${ }^{\circledR}$ (for IQ BB waveform capture).

\subsection{Future Work}

Spectrum Sensing and synchronization are major challenges in HetNet (Heterogeneous Network) and in SON (Self Organizing Network) networks. Also, carrier aggregation is one of the most important technologies in the new LTE-Advanced standards. This technique will also play a significant role for $4 \mathrm{G}$ communication systems. The task of carrier aggregation is inherent in the cognitive radio world, i.e. the manipulation of OFDM waveforms to fill an available bandwidth. Due to the lack of resources available (IQCreator ${ }^{\mathrm{TM}}$ LTE-TDD waveform creation software was not available) and the time constraints, LTETDD synchronization was not investigated in this thesis. This requires further study and investigation. In addition, there is a current surge in the demands and momentum for LTE-TDD duplexing techniques. 


\section{References}

[1] Chang R W., "Synthesis of band-limited orthogonal signals for multichannel data transmission ", Bell Syst. Tech. J., vol. 46, pp. 1775-1796, 1966 .

[2] Peled A., Ruiz A., "Frequency Domain Data Transmission Using Reduced Computational Complexity Algorithms," in Proceedings of the IEEE International Conference on Acoustics, Speech, and Signal Processing (ICASSP'80), pp. 964-967, 1980.

[3] Urkowitz H., "Energy detection of unknown deterministic signals," Proc. IEEE, vol. 55, no. 4, pp. 523-531, 1967.

[4] North D.O., "An Analysis of the Factors Which Determine Signal/Noise Discrimination in PulsedCarrier Systems," RCA Laboratories, Princeton, N. J., Report FTR-6C, June, 1943.

[5] Gardner W.A., "Exploitation of Spectral Rredundancy in Cyclostationary Signals", IEEE Signal Processing Mag., vol. 8, no. 2, pp. 14-36, 1991.

[6] Schmidl T. and Cox D., "Robust frequency and timing synchronization for OFDM". IEEE Transactions on Communications, 45(12):1613-1621, Dec. 1997.

[7] Van de Beek J., Sandell M., and Borjesson P., "ML estimation of time and frequency offset in OFDM systems," IEEE Trans. Signal Processing, vol. 45, no. 7, pp. 1800-1805, July 1997.

[8] Minn H., Zeng M., and Bhargava V. K., “On Timing Offset Estimation for OFDM Systems" IEEE Communications Letters, VOL. 4, NO. 7, July 2000, pp 242-244.

[9] Park B., Cheon H., Kang C., and Hong D., "A Novel Timing Estimation Method for OFDM Systems", IEEE Communications Letters, VOL. 7, NO. 5, MAY 2003, pp.239-241.

[10] Shi K., Serpedin E., "Coarse Frame and Carrier Synchronization of OFDM Systems: A New Metric and Comparison", IEEE Transactions on Wireless Communications, 2004, 3(4): 1271-1284.

[11] M. L. Moher and J. H. Lodge, "TCMP-A modulation and coding strategy for Rician fading channels," IEEE J. Select. Areas Commun., vol. 7, pp. 1347-1355, Dec. 1989.

[12] IEEE Std 802.16, “Air Interface for Broadband Wireless Access Systems”, May 2009. 
[13] IEEE 802.22, "Cognitive Wireless RAN Medium Access Control (MAC) and Physical Layer (PHY) specifications: Policies and procedures for operation in the TV Bands", July 2011.

[14] IEEE Std P1900.6, "IEEE Standard for Spectrum Sensing Interfaces and Data Structures for Dynamic Spectrum Access and Other Advanced Radio Communication Systems", April 2011.

[15] Simon Haykin, "Cognitive radio: Brain-empowered wireless communications", IEEE Journal on Selected Areas in Communications, vol. 23, no. 2, pp. 201-220, February 2005.

[16] Q. Wang, C. Mehlfuihrer, and M. Rupp, "Carrier frequency synchronization in the downlink of 3GPP LTE", in Proc. PIMRC, 2010, pp.939-944.

[17] Yong Soo Cho, Jaekwon Kim, Won Young Yang, Chung G. Kang, "MMMO-OFDM Wireless Communications with MATLAB”, John Wiley \& Sons, November 2010.

[18] Won Y. Yang, Yong S. Cho, Won G. Jeon, Jeong W. Lee, Jong H. Paik, Jae K. Kim, Mi-Hyun Lee, Kyu I. Lee, Kyung W. Park, Kyung S. Woo, "MATLAB/Simulink for Digital Communication", A-Jin Publishing, 2009.

[19] Ye Li, Gordon L. Stüber, "Orthogonal Frequency Division Multiplexing for Wireless Communications", Springer 2006.

[20] Thomson, D. J. "Spectrum estimation and harmonic analysis", Proceedings of the IEEE, Volume 70 (1982), 1055-1096.

[21] Classen F., Meyr H., "Frequency Synchronization Algorithms for OFDM Systems suitable for Communication over Frequency Fading Channels", IEEE Veh. tech. Conf., vol. 3, 1994, pp. 1655-1659.

[22] Rappaport, T.S., "Wireless Communications Principle and Practice", Prentice Hall, 2002.

[23] Setiawan H., Kurosaki M., Ochi H., " LTE Physical Layer Identity Detection: Frequency vs Time Domain Schemes", 2011 IEEE International Conference on Communications (ICC), 29 July 2011.

[24] Fazel K., Kaiser S., "Multi-Carrier and Spread Spectrum Systems From OFDM and MC-CDMA to LTE and WiMAX", Second Edition, John Wiley \& Sons, 2008.

[25] M. Morelli and U. Mengali, "An improved frequency offset estimator for OFDM applications," IEEE Commun. Lett., vol. 3, no. 3, pp. 75-77, 1999. 
[26] Y. Hur, J. Park, W. Woo, K. Lim, C.-H. Lee, H. S. Kim, and J. Laskar, "A wideband analog multiresolution spectrum sensing technique for cognitive radio systems", IEEE International Symposium on Circuits and Systems (ISCAS'06), pp. 4090-4093, May 2006.

[27] Y. Zeng and Y. Liang, "Covariance based signal detections for cognitive radio," in 2nd IEEE DySPAN, Dublin, Ireland, 2007, pp. 202-207.

[28] B. Farhang-Boroujeny, “Filter bank Spectrum Sensing for Cognitive Radios", IEEE Trans. on Signal Processing, vol. 56, no. 5, pp. 1801-1811, May 2008.

[29] P.H. Moose [1994], "A Technique for Orthogonal Frequency Division Multiplexing Frequency Offset Correction," IEEE Transactions on Communications, 42, 10, pp. 2908-2914.

[30] R. Haas, "Applications of multicarrier modulation in mobile radio communications", PhD thesis, Ecole Nationale Superieure des Telecommunications, Paris, 1996.

[31] J. Andrews, A.Ghosh, R. Muhamed, "Fundamentals of WiMAX: Understanding Broadband Wireless Networking", Prentice-Hall, 2007.

[32] K. Arshad, S. Chantaraskul, X. Gelabert, C. Germond, J. Kronander, M. I. Rahman, A. Richter, O. Sallent, Y. Selén, E $E^{3}$ White Paper Spectrum Sensing, November 2009.

[33] 3GPP TS 36.211: “Technical Specification Group Radio Access Network; Evolved Universal Terrestrial Radio Access (E-UTRA); Physical Channels and Modulation", V10.4.0, December 2011.

[34] Furht B., Ahson S. A., Long Term Evolution: 3GPP LTE Radio and Cellular Technology, CRC Press, April 2009.

[35] T. Yucek and H. Arslan, "A Survey of Spectrum Sensing Algorithms for Cognitive Radio Applications", IEEE Communication Surveys \& Tutorials, Vol.11, No.1, pp 116-130, 2009.

[36] M. Morelli, C.-C. Jay Kuo, Pun, M.-O., Man-On Pun, "Synchronization Techniques for Orthogonal Frequency Division Multiple Access (OFDMA): A Tutorial Review", Proceedings of the IEEE, vol.95, no.7, pp.1394-1427, July 2007.

[37] Sklar, B., "Digital Communications: Fundamentals and Applications", $2^{\text {nd }}$ edition, Prentice Hall, January 2001. 


\section{Appendix A. LTE-OFDM Transceiver System Setup}

Below is a pictorial representation for LTE OFDM $1 / Q$ waveform creation, generation, transmission, reception, and processing.

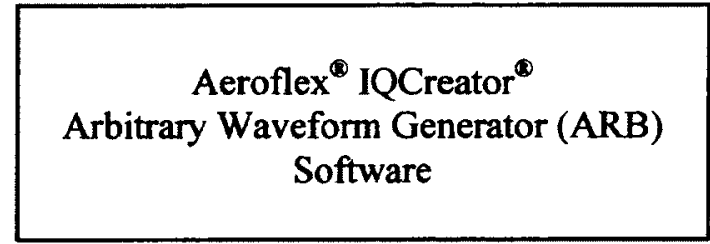

(1) \& (2)

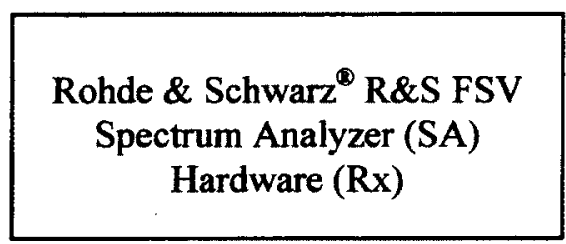

(6)

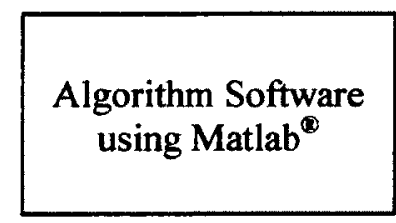

(10)

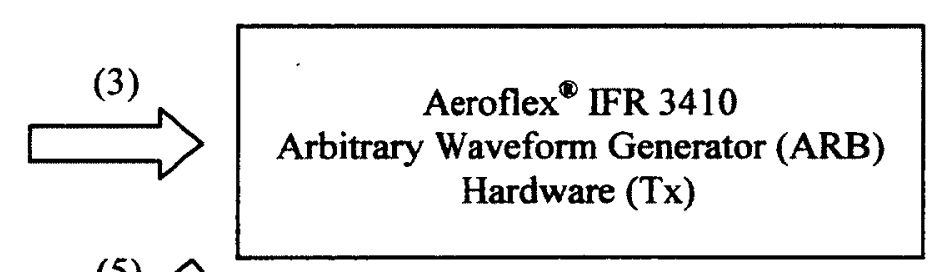

(4)
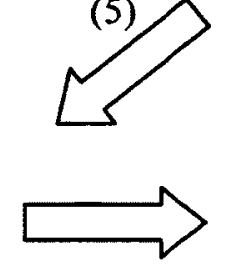

(7)

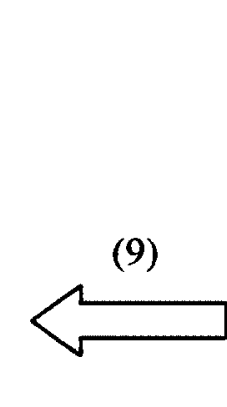

LTE-OFDM I/Q Waveform Files

\section{A.1 LTE Complex Signal I/Q Waveform File Generation Procedure}

1) Create and configure LTE-OFDM parameters (*.iqc) file using ARB software.

2) Generate LTE Baseband signal (*.aiq) file

3) Download LTE Baseband signal (*.aiq) file to ARB hardware.

4) Upconvert and generate LTE RF modulated signal file using ARB hardware.

5) Feed LTE RF modulated signal file into spectrum analyzer hardware.

6) Downconvert and generate LTE baseband V/Q signal file

7) Capture LTE baseband I/Q signal file using SA software.

8) Save LTE I/Q signal (*.das) file using SA software.

9) Load LTE babeband I/Q waveform file into Matlab software.

10) Estimate symbol/frequency offset using STO/CFO synchronization algorithm code. 


\section{Appendix B. LTE/LTE-A Cell Search Process}

As soon as the receiver starts up, it must search for the presence of OFDM symbols in the received signal. Usually, symbol detection exploits some form of repetition in the received OFDM signal. This can be the cyclic prefix inserted in the guard interval or the preambles consisting of identical periods. In contrast to packet-oriented networks, LTE is a frame-oriented network and does not employ a PHY preamble to facilitate carrier offset estimate, channel estimation, timing synchronization etc. Instead, special reference signals are embedded in the PRBs (Physical Resource Blocks). Reference signals are transmitted during the first and fifth OFDM symbols of each slot when the short CP is used and during the first and fourth OFDM symbols when the long CP is used.

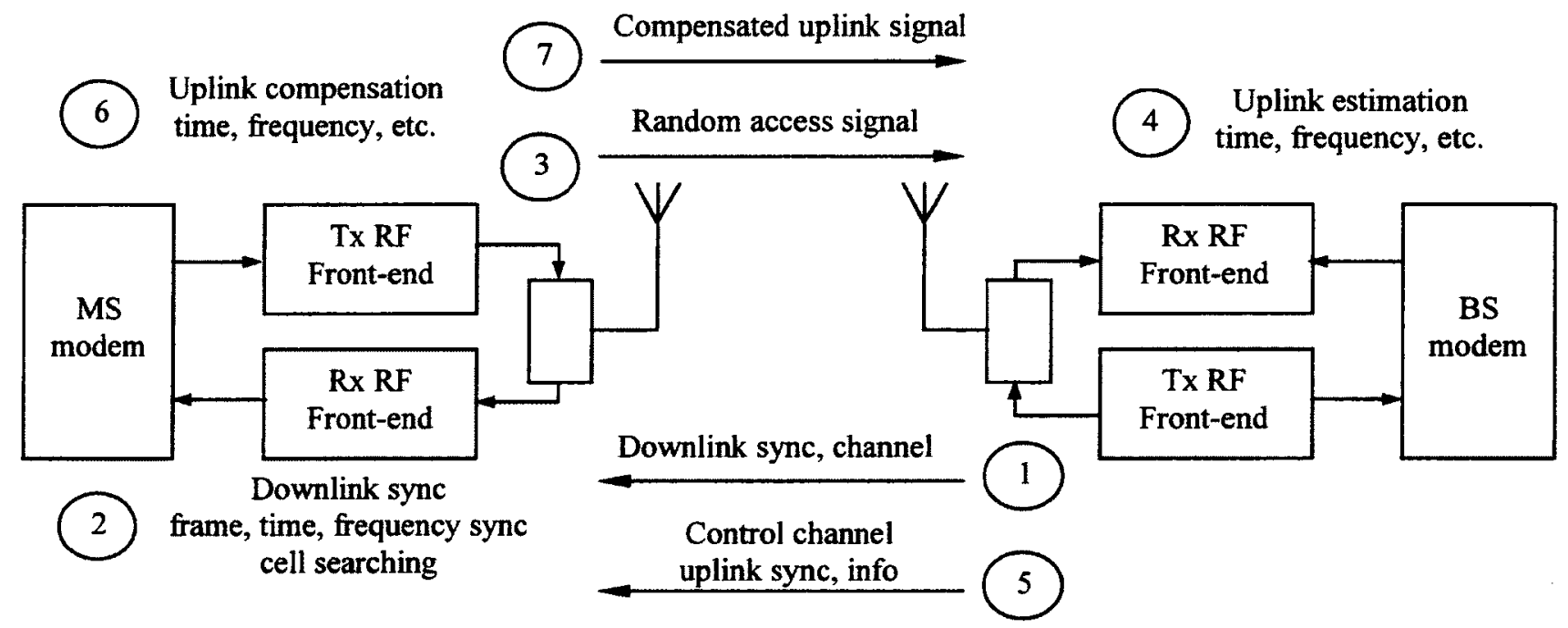

\section{A.2 Cell Search and Synchronization Process in LTE/LTE-A}

The cell search and synchronization process consists of the following steps [17]:

1) The Base station broadcasts a downlink preamble periodically $\mathrm{P}-\mathrm{SCH}$ and $\mathrm{S}-\mathrm{SCH}$, which can be used for maintaining a connection to any Base station within the vicinity (alignment in time and frequency).

2) The Mobile station acquires the frame timing, symbol timing, carrier frequency, and Cell ID by using the preamble transmitted from the Base station. Base station also acquires information on timing and resource for random access. 
3) The Mobile station transmits a random access preamble using the resource information (time, frequency, code) broadcasted by Base station.

4) Upon reception of the random access preamble, the Base station estimates the uplink symbol timing offset, carrier frequency offset (only for FDD case), and power level for the Base station.

5) The Base station sends a ranging response (as a response to the random access $\mathrm{RACH}$ ) indicating the value of timing advance (TA), carrier frequency offset, and power level for the Base station.

6) The Mobile station compensates the uplink timing, carrier frequency offset, and power level by using the information in the ranging response.

7) The Mobile station transmits a compensated uplink signal. 


\section{Appendix C. STO, CFO Estimates, and MSE Data Results}

Below are STO, CFO estimates, as well as the Mean Square Error (MSE) performance experimental results for the cyclic prefix-based, preamble-based, and pilot-based methods using normal and extended cyclic prefix with LTE bandwidths $1.4,10$, and $20 \mathrm{MHz}$ respectively.

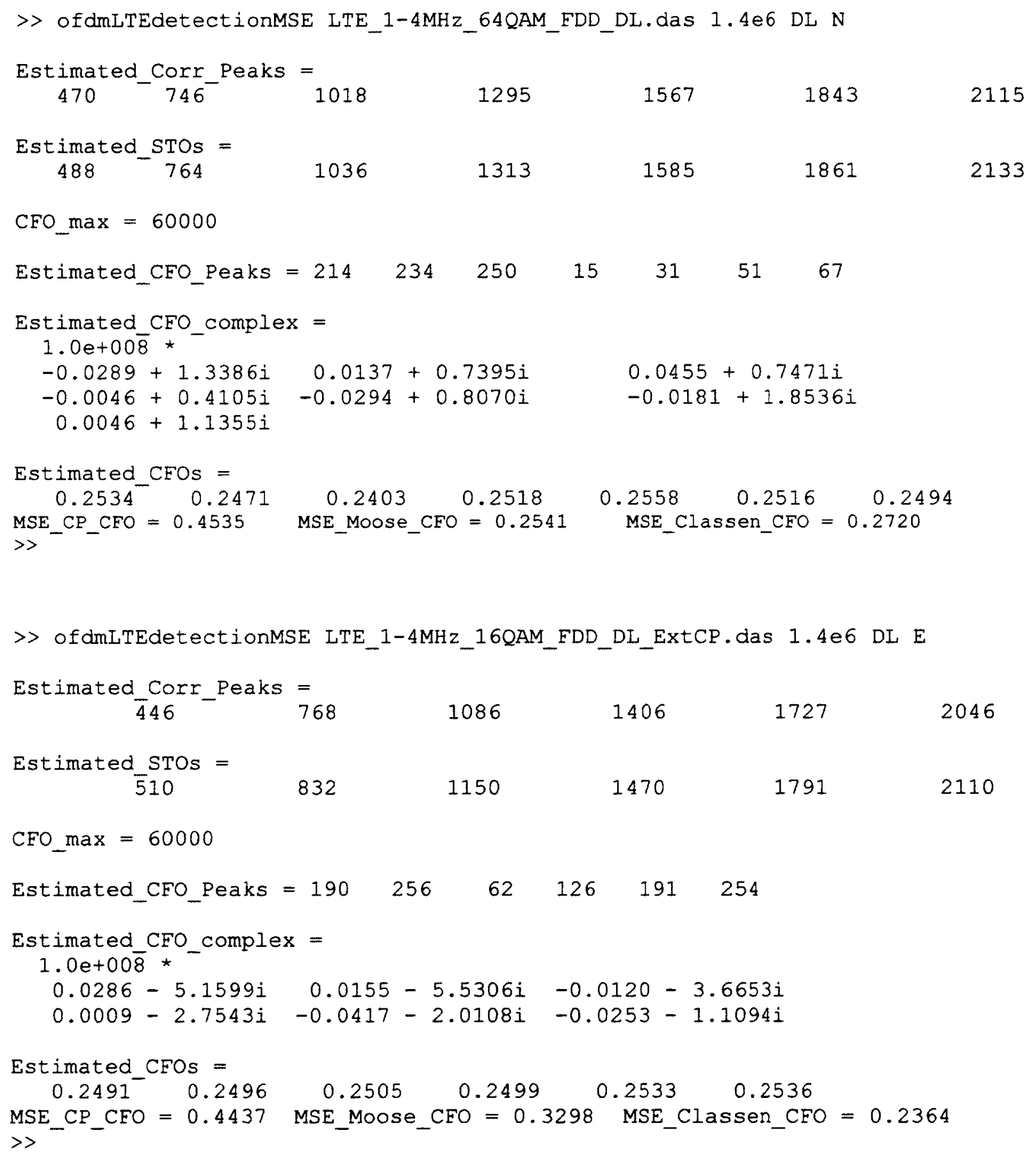




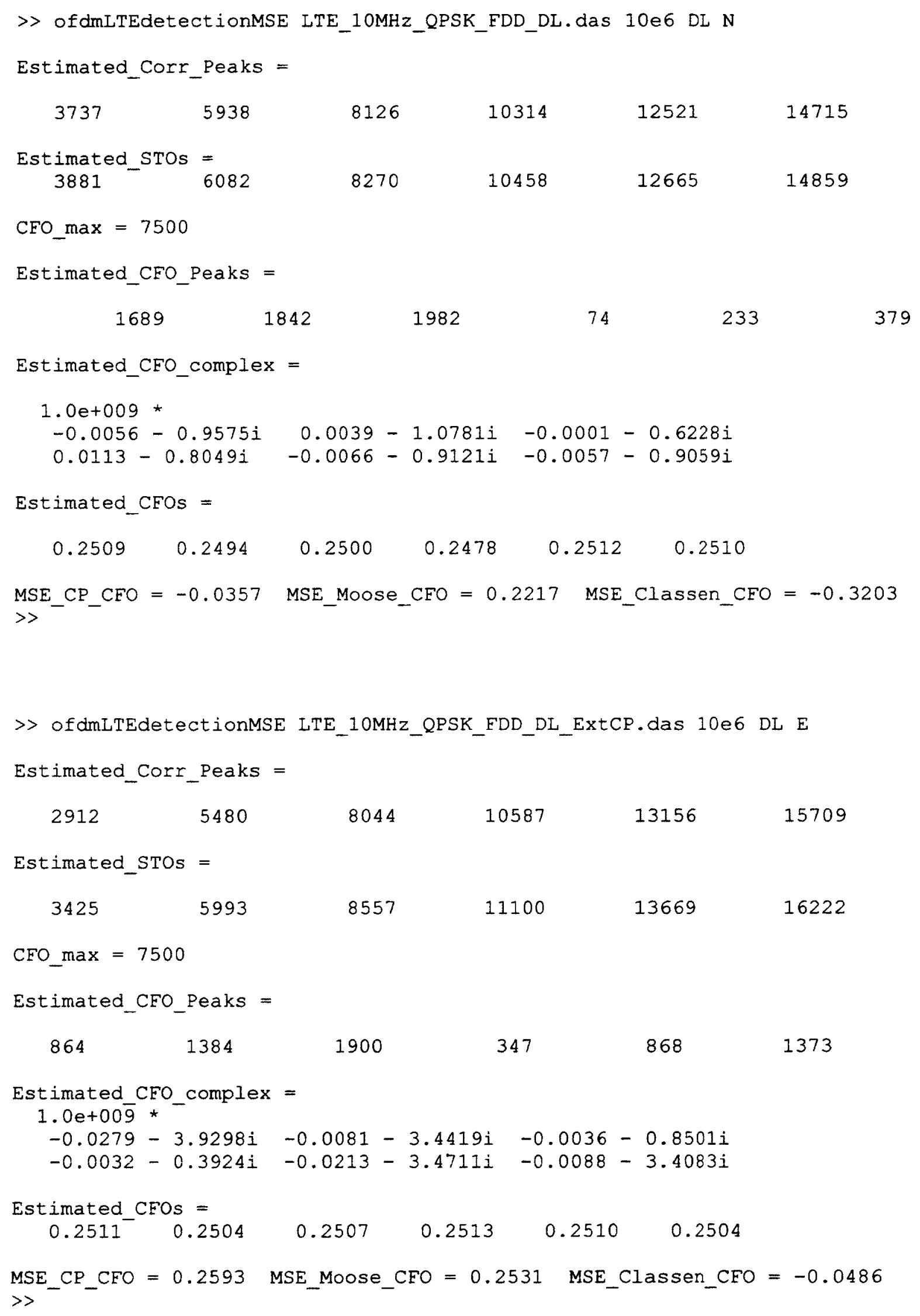




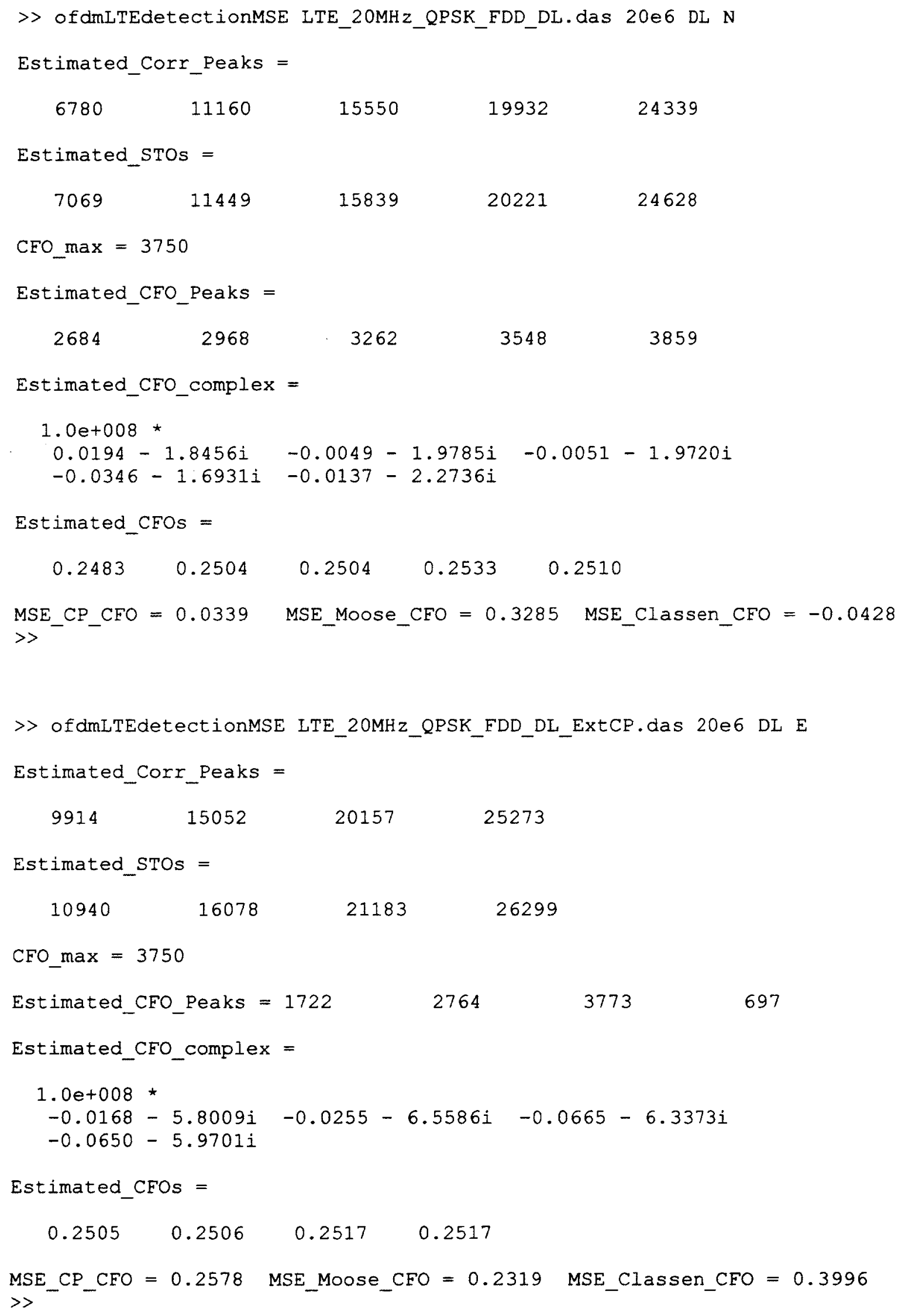

